\title{
Reconstitution and functional characterization of efflux pumps from Escherichia coli
}

\author{
Dissertation \\ zur Erlangung des Doktorgrades \\ der Naturwissenschaften \\ vorgelegt im Fachbereich 14 Biochemie, Chemie und Pharmazie \\ der Johann Wolfgang Goethe - Universität \\ in Frankfurt am Main \\ von \\ Selena Đorđević-Marquardt \\ aus Pirot, Serbien
}

Frankfurt am Main (2021)

(D30) 
Vom Fachbereich Biochemie, Chemie und Pharmazie (FB 14)

der Johann Wolfgang Goethe - Universität als Dissertation angenommen.

Dekan: Prof. Dr. Clemens Glaubitz

Gutachter: Prof. Dr. Klaas Martinus Pos

Prof. Dr. Clemens Glaubitz

Datum der Disputation: 
"If you want to find the secrets of the universe, think in terms of energy, frequency and vibration.',

Nikola Tesla 


\section{$\underline{\text { Table of Contents }}$}

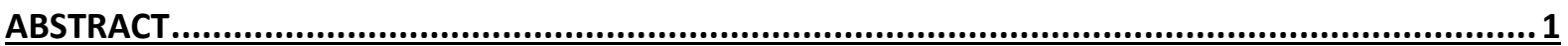

ZUSAMMENFASSUNG . . .4

1. CHAPTER I: INTRODUCTION.. .10

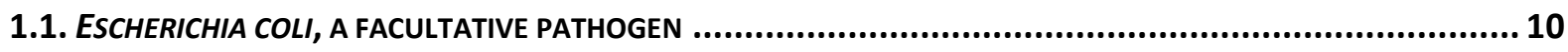

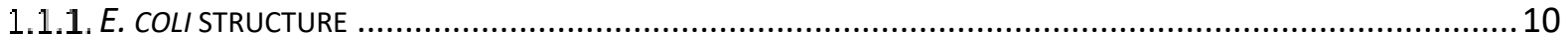

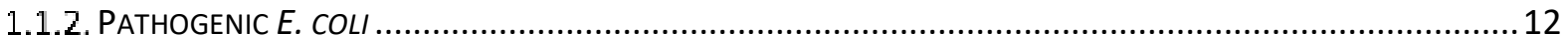

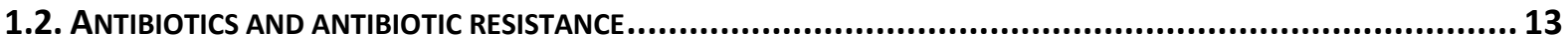

1.2.1. ANTIBIOTICS AND THEIR TARGETS - MECHANISM OF ACTION ............................................... 14

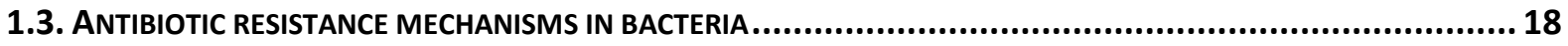

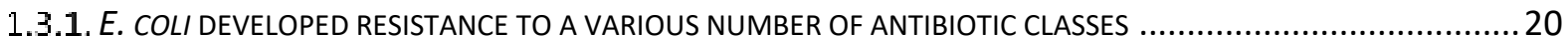

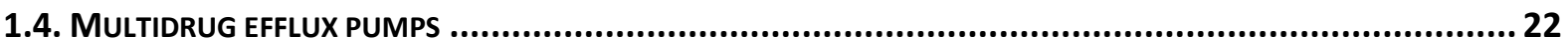

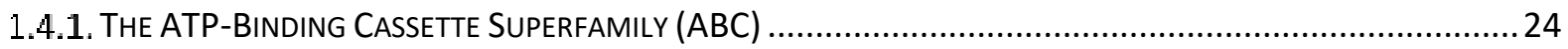

1.4.2. The MultidRug ANd TOXIC COMPOUNd EXTRUsion SUPERFAMILY (MATE) AND THE PROTEOBACTERIAL

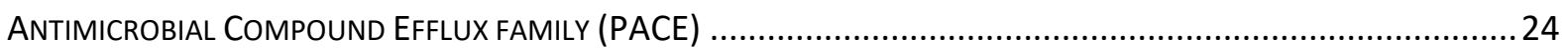

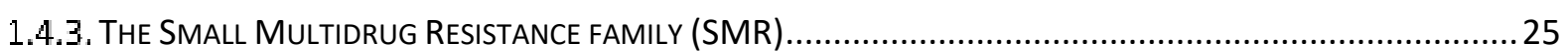

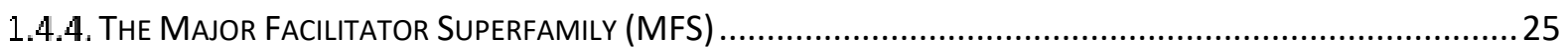

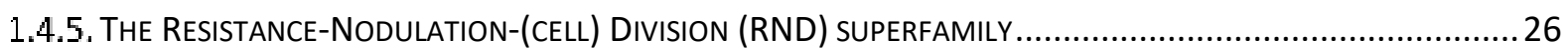

1.5. Metal-ION ReSiStANCE IN E. COLI ........................................................................ 27

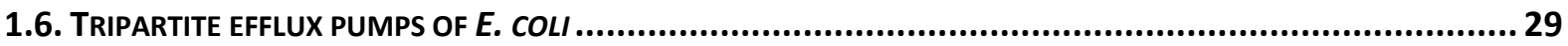

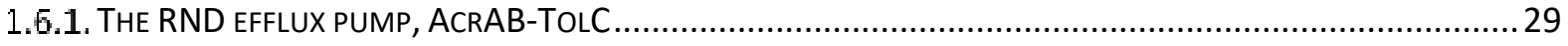

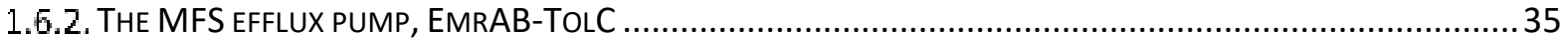

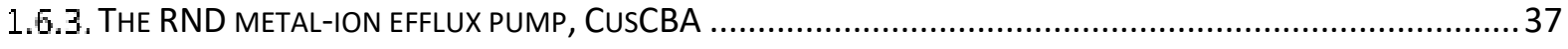

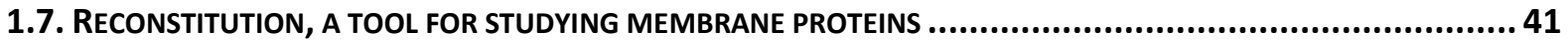

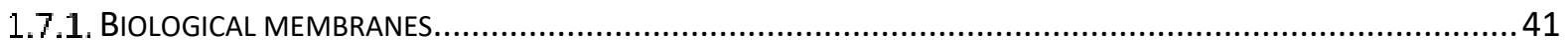

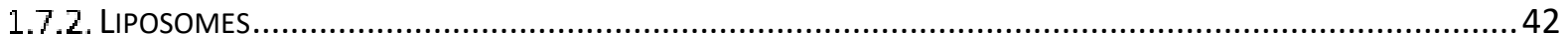

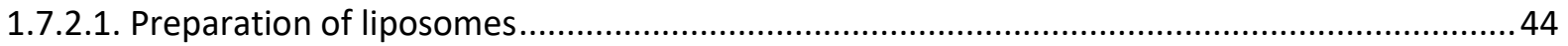

1.7.2.2. Applications of liposomes in medicine and pharmacology ..........................................45

1.7.3. RECONSTITUTION OF MEMBRANE PROTEINS IN LIPID VESICLES ...............................................46

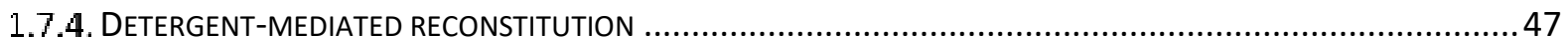




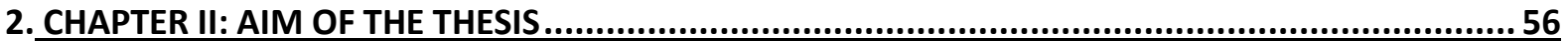

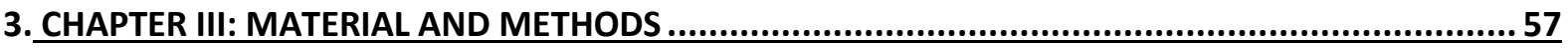

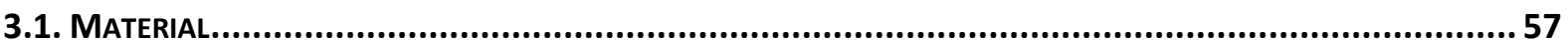

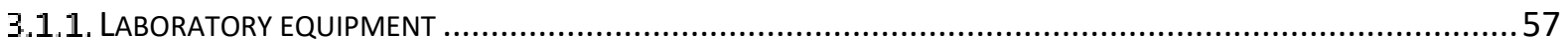

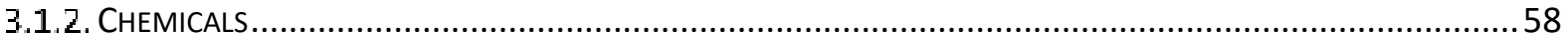

3.1.3. BACTERIAL STRAINS: FOR MOLECULAR BIOLOGY TECHNIQUES, FOR PROTEIN PRODUCTION, FOR FUNCTIONAL

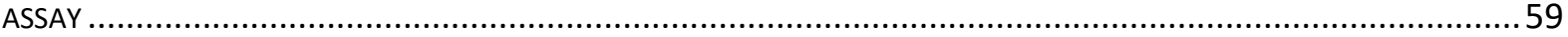

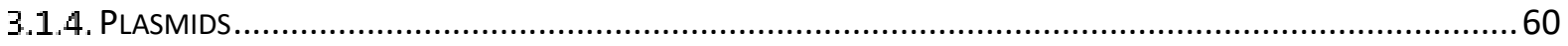

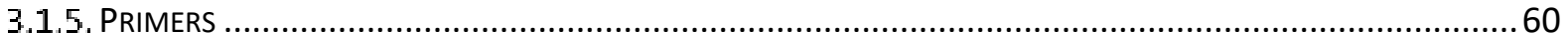

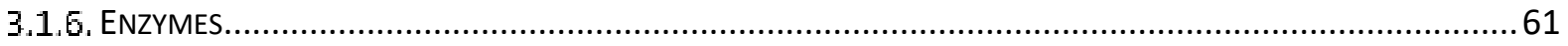

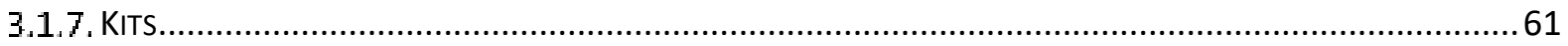

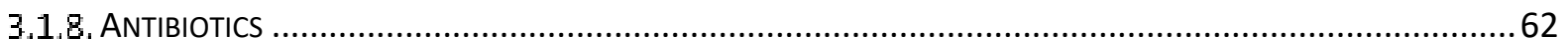

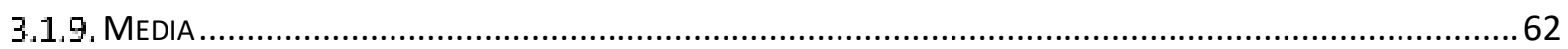

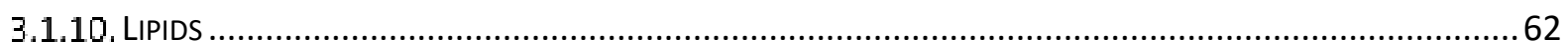

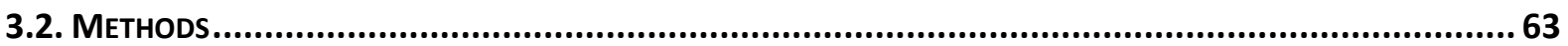

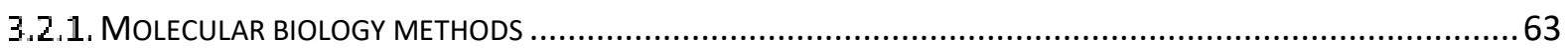

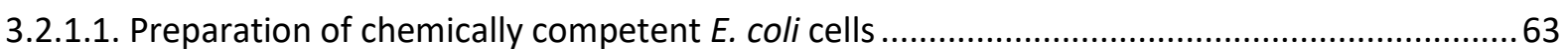

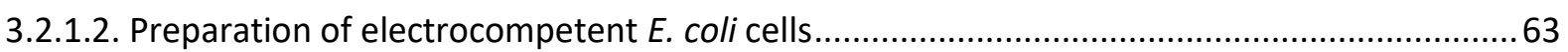

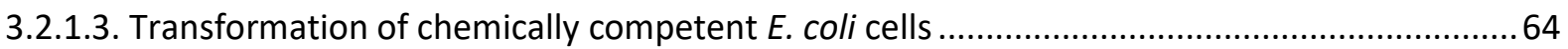

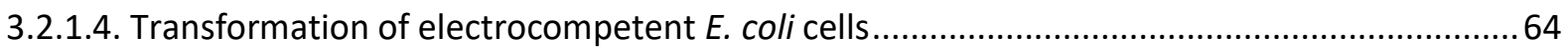

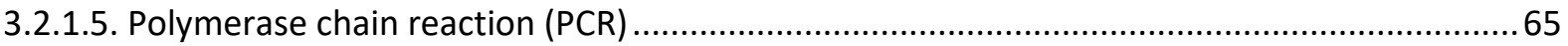

3.2.1.6. Agarose gel electrophoresis; extraction of DNA fragments from agarose gel .......................66

3.2.1.7. Photometric determination of the concentration of DNA and DNA sequencing ....................66

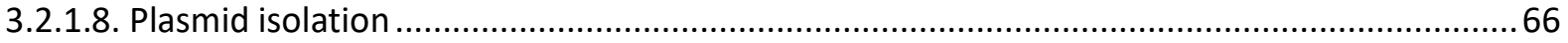

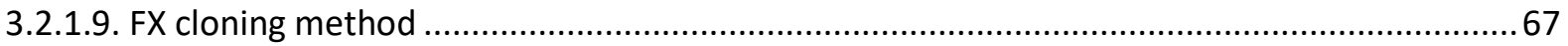

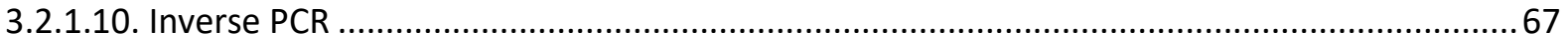

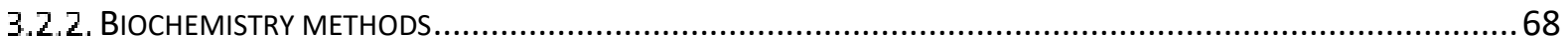

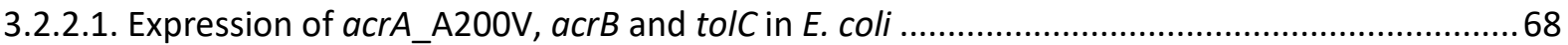

3.2.2.2. Purification of AcrA A200V, AcrB wild type, AcrB D407N and TolC .....................................68

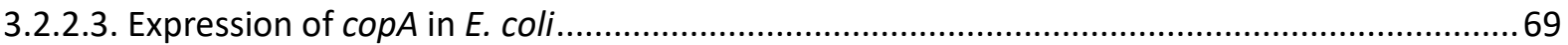




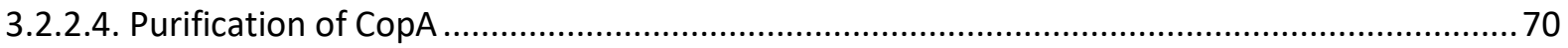

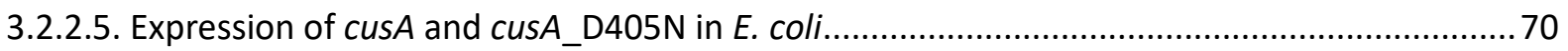

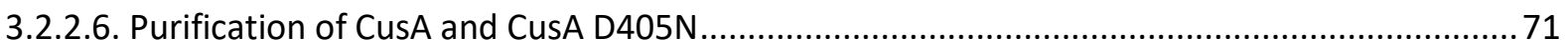

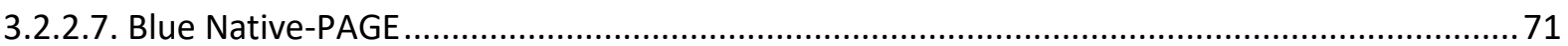

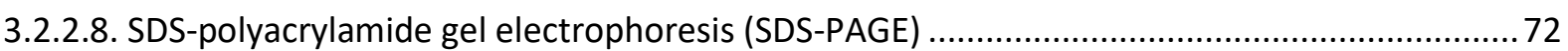

3.2.2.9. Preparation of the samples for SDS-PAGE: whole cells, solubilized cells, protein purification .73

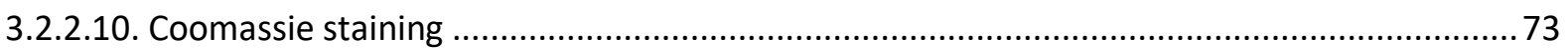

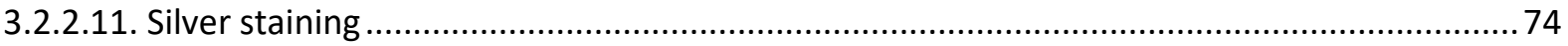

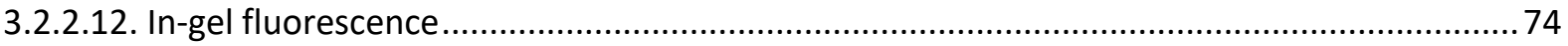

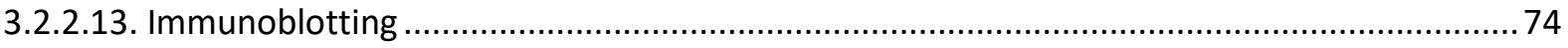

3.2.2.14. Determination of the protein concentration ...................................................................... 75

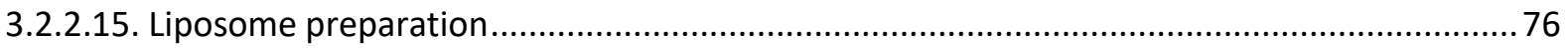

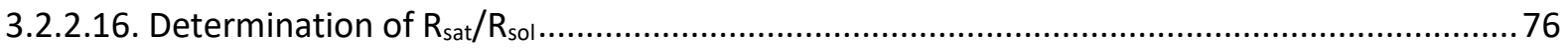

3.2.2.17. Reconstitution of AcrA A200V, AcrB, AcrB D407N, AcrB F628L and TolC in LUVs................ 77

3.2.2.18. Reconstitution of CopA, CusA wild type and CusA D405N in LUVs .................................... 78

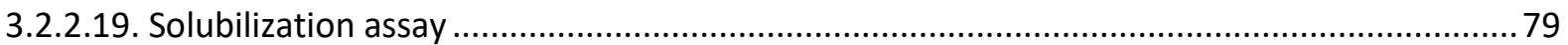

3.2.2.20. Pyranine fluorescence and $\mathrm{pH}$ dependence; PhenGreen SK titration with silver ions; TolC

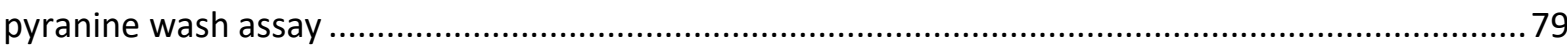

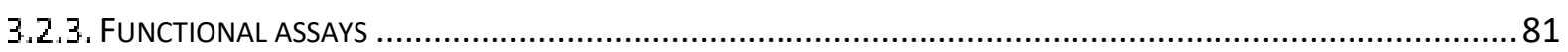

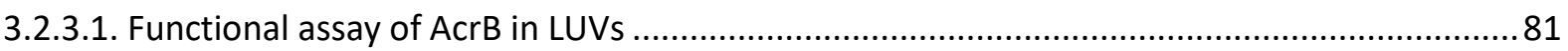

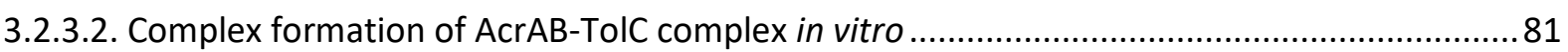

3.2.3.3. Determination of the Michaelis-Menten constant $\left(K_{m}\right)$ for ATP of CopA.............................. 82

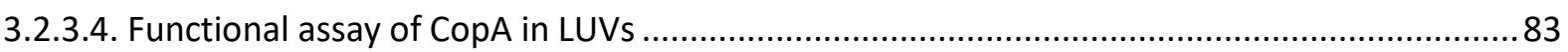

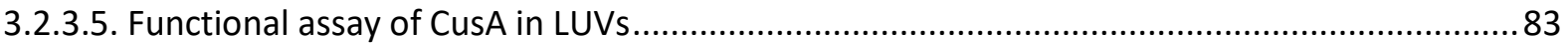

3.2.3.6. Drug agar plate dilution assay for testing the activity of EmrAB-TolC efflux pump ................ 84

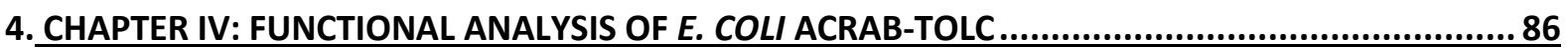

4.1. INTRODUCTION .86

4.1.1. THE TRANSPORT OF SUBSTRATES RESULTS IN ACRB-MEDIATED PROTON INFLUX, WHEN ACRB IS RECONSTITUTED

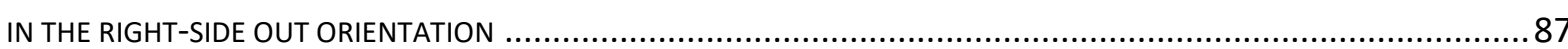

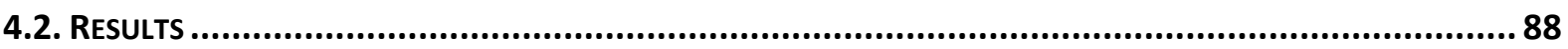

4.2.1. PYRANINE AS A REPORTER SYSTEM FOR OBSERVATIONS OF PROTON FLUX IN THE PROTEOLIPOSOME ASSAY ....... 88 
4.2.2. PRODUCTION OF PROTEINS FOR IN VITRO ASSAYS .90

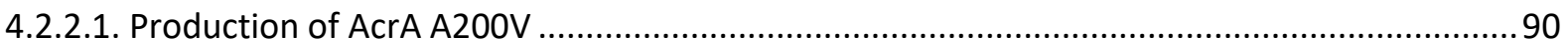

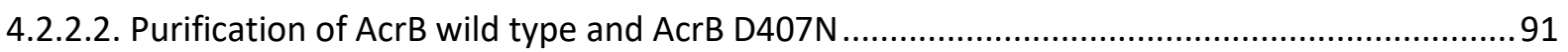

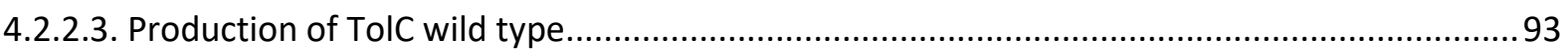

4.2.3. THE BLUEPRINT OF THE PROTEOLIPOSOME ASSAY - DETECTION OF PERIPLASMIC DRUG TRANSPORT BY ACRB...94

4.2.3.1. The activity of AcrB in the presence of MBX3132 and Phe-Arg $\beta$-naphthylamide (PAßN).....98

4.2.3.2. The $\mathrm{H}^{+}$-transport activity of the MBX3132-insensitive variant, AcrB F628L ........................102

4.2.4، IN VITRO ASSEMBLY OF THE EFFLUX SYSTEM ACRAB-TOLC IS INDEPENDENT OF THE PRESENCE OF SUBSTRATE 103 4.2.4.1. MagStrep-mediated pull-down of proteoliposomes as a tool for detection of the tripartite

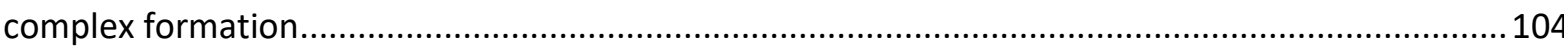

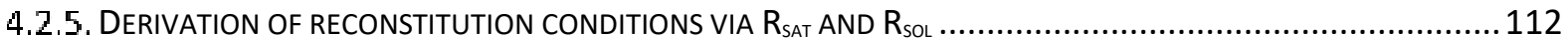

4.2.6. SOLUBILIZATION ASSAY TO VALIDATE THE RECONSTITUTION OF MEMBRANE PROTEINS INTO ARTIFICIAL MEMBRANES.

4.2.7. RECONSTITUTION EFFICIENCY AND ESTIMATIONS OF PROTEIN AMOUNT INCORPORATED IN THE LIPOSOMES...116 TOLC - THE OUTER MEMBRANE PROTEIN OF THE ACRAB-TOLC SYSTEM

4.3. DISCUSSION AND CONCLUSION 120

4.3.1. PYRANINE AS A REPORTER SYSTEM 120

4.3.2. DESIGNING AN IN VITRO ASSAY FOR MONITORING H ${ }^{+}$-TRANSPORT OF ACRB IN AN ARTIFICIAL MEMBRANE ....121

4.3.3. USAGE OF STREPTAVIDIN-BIOTIN BOND FOR MONITORING ASSEMBLY OF THE EFFLUX PUMPS ..... .124

4.3.4. TOLC WILD TYPE IN A CLOSED STATE IS AN ESSENTIAL PART FOR STUDYING THE SUBSTRATE TRANSPORT ........126

4.3.5. EFFICIENCY OF THE PROTEIN INCORPORATION INTO THE MEMBRANE. 128

\section{CHAPTER V: SILVER-ION RESISTANCE IN ESCHERICHIA COLI IS MEDIATED BY THE EFFLUX PUMPS COPA AND CUSCBA

5.1. The USE OF P-TYPe ATPASE COPA AS PROOF-OF-PRINCIPLE FOR SILVER-ION TRANSPORT OBSERVATIONS .... 130

5.1.1. INTRODUCTION 130

5.1.2. RESULTS 131

5.1.2.1. Production of CopA wild type for the functional analysis 131

5.1.2.2. CopA ATPase activity. 132

5.1.2.3. Phen Green SK ${ }^{\mathrm{TM}}$ as a reporter system 134

5.1.2.4. Solubilization assay to validate the reconstitution of CopA wild type into artificial membranes 
5.1.2.5. Phen Green SK as a reporter system for the transport of silver ions by the P-type ATPase CopA

5.1.3. DISCUSSION

5.1.3.1. Produced CopA is active in detergent micelles.

5.1.3.2. CopA wild type shows active transport activity towards silver ions.

5.1.3.3. Reconstitution with freeze-thaw procedure

5.2. THE tRIPARTITE Cu(I) AND Ag(I) EFFLUX PUMP CUSCBA CAN SEQUESTER METAL IONS FROM THE PERIPLASM

AND FROM THE CYTOPLASM

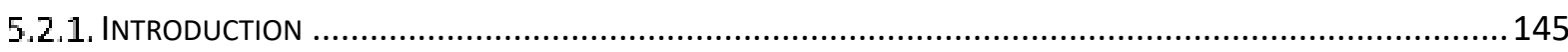

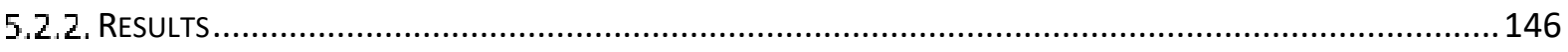

5.2.2.1. Production of CusA wild type for reconstitution and transport assays................................146

5.2.2.2. Production of CuSA D405N variant for reconstitution and transport assays ....................... 147

5.2.2.3. Solubilization assay to validate the reconstitution of CusA wild type into artificial membranes

5.2.2.4. Different orientations of CusA in liposomes can be triggered by the application of different

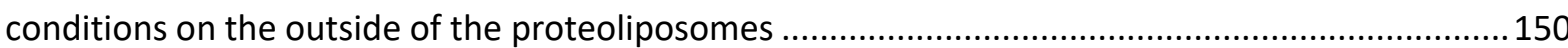

5.2.2.5. ACMA as a reporter system of the right-side out oriented CusA in liposomes. 151

5.2.2.6. PhenGreen SK dye as a reporter system of $\mathrm{Ag}^{+}$-transport by CusA reconstituted in liposomes 158

5.2.3. DISCUSSION 162

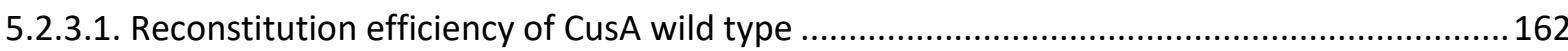

5.2.3.2. CusA wild type reconstituted into liposomes shows activity ..............................................163

5.2.3.3. Transport of silver ions by CusA wild type proteoliposomes ...............................................168

\section{CHAPTER VI: FUNCTIONAL ANALYSIS OF A TRIPARTITE MAJOR FACILITATOR SUPERFAMILY}

(MFS)-TYPE EFFLUX PUMP EMRAB-TOLC FROM E. COLI ..................................................... 172

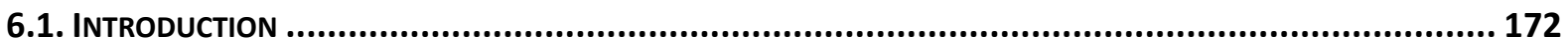

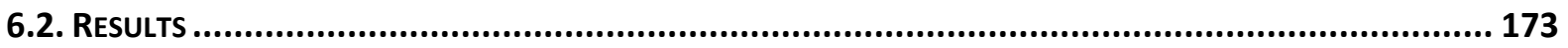

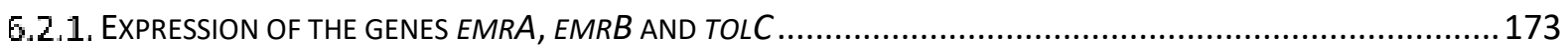

6.2.2. TESTING OF THE E. COLI STRAINS FOR THE OPTIMIZATION OF THE SUBSTRATE CONCENTRATION OF CARBONYL

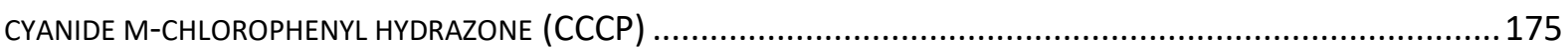

6.2.3. CO-EXPRESSION OF FUSION PROTEINS EMRB-MRFP AND EMRA-TOLC-GFP FORMS A FUNCTIONAL TRIPARTITE SYSTEM. 
6.2.4. A DRUG SUSCEPTIBILITY ASSAY OF E. COLI EXPRESSION STRAIN, C41(DE3), HARBOURING GENES EXPRESSING THE

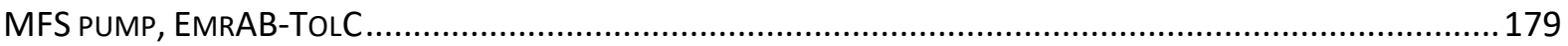

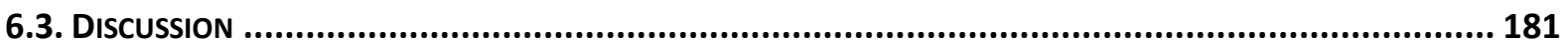

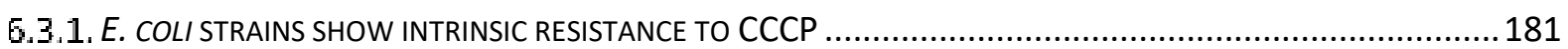

6.3.2. CELLS EXPRESSING THE MFS COMPONENT SHOW MORE SUSCEPTIBILITY TOWARDS CCCP COMPARED TO CELLS

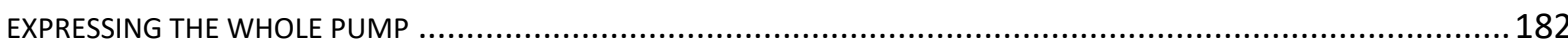

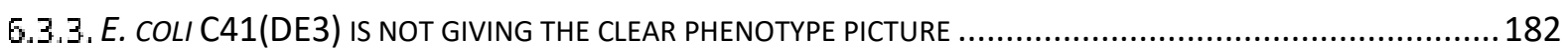

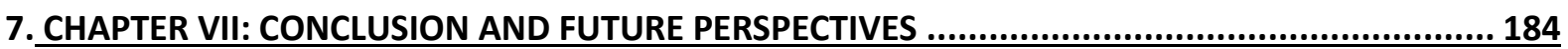

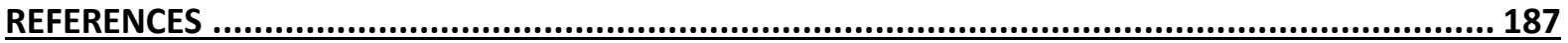

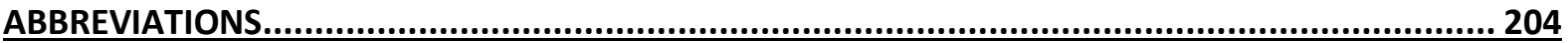

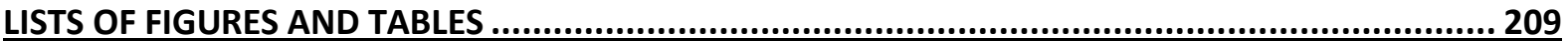

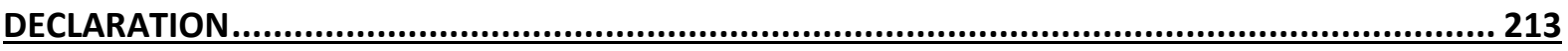

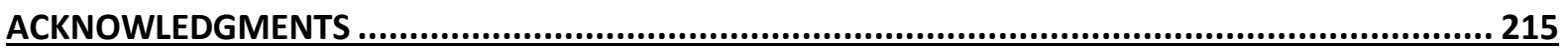

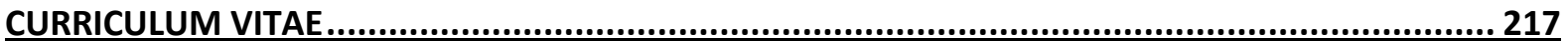




\section{$\underline{\text { Abstract }}$}

Resistant microbes are a growing concern. It was estimated that about 33,000 of people die because of the infections caused by multidrug resistant bacteria each year in Europe (ECDC, 2018, https://www.ecdc.europa.eu/). Bacteria can acquire resistance against toxic compounds via different mechanisms and intrinsic active efflux is one of the first mechanisms deployed by bacterial cells. The membrane-localized efflux pumps catalysing this reaction, extract toxic compounds from the interior of the cell and transport these to the outside, thereby maintaining sub-lethal toxin levels in the cytoplasm, periplasm and membranes. Gramnegative three-component efflux pumps, analysed in this study, are composed of an inner membrane protein, a member of the Resistance-Nodulation cell Division (RND) superfamily, an Outer Membrane Factor (OMF) protein and a Membrane Fusion Protein (MFP) that connects the two afore mentioned components into an active efflux pump. The pumps described in this work, AcrAB-TolC and EmrAB-TolC, are drug efflux pumps belonging to the RND and MFS superfamilies, respectively, while CusCBA is an efflux pump that belongs to the RND heavy metal efflux family. Another efflux pump that was used as a model for the design of an in vitro assay for the silver ion transport studies, CopA, belongs to the P-type ATPase superfamily. All pumps analysed in this study are part of the resistance system of Escherichia coli, which is a highly clinically relevant pathogen.

In order to examine the AcrAB-TolC, CopA and CusA efflux pumps, the individual components were separately produced in E. coli, purified to monodispersity and reconstituted in large unilamellar vesicles, LUVs. Means for the optimized production and adequate conditions for efficient reconstitution were presented in this study. The activity of AcrB in LUVs was detected using fluorescence quenching of the dye 8-hydroxy1,3,6-pyrenetrisulfonate (pyranine), which is incorporated inside the proteoliposomes and is sensitive to the $\mathrm{pH}$ changes in its surrounding. The inactive AcrB variant with a substitution in the proton relay network, D407N, showed no activity in proteoliposomes, which correlates with the measurements done in empty liposomes. When AcrA was co-reconstituted with AcrB D407N proteoliposomes it did not restore protein activity. To test the assembly of the AcrAB-TolC pump out of its single components, an in vitro assay was established where the complex assembly was tested with AcrAB- and TolC-containing liposomes. These experiments showed putative AcrAB-TolC formation in the presence or absence of a pump 
substrate, taurocholate, as well as in the presence of the pump inhibitor, MBX3132. The assembly appeared stable over time and results were invariant in the presence or absence of a $\mathrm{pH}$ gradient across the AcrAB-containing membrane.

After determination of the ATPase activity of the P-type ATPase, CopA, in detergent micelles, the protein was reconstituted in LUVs. Quenching of the $\mathrm{Ag}^{+}$-sensitive dye Phen Green SK (PGSK), present on the inside of the CopA-containing proteoliposomes, was observed in presence of ATP and $\mathrm{Ag}^{+}$. Under the same conditions, but in absence of $\mathrm{Ag}^{+}-$ ions, quenching was reduced by $80 \%$ after 300 seconds. No PGSK-quenching was observed in control liposomes in the presence of ATP and $\mathrm{Ag}^{+}$. The additional presence of sodium azide led to minimal reduction of the PGSK-quenching as expected since sodium azide is not an inhibitor of P-type ATPases, but the quenching rate was similar to that of the same experimental condition with control liposomes.

The RND superfamily member CusA, as part of the tripartite CusCBA efflux pump, has been proposed to sequester $\mathrm{Ag}^{+}$or $\mathrm{Cu}^{+}$from either the cytoplasmic or periplasmic side of the inner membrane. The periplasmic transport of silver ions was implied from an in vitro assay where the quenching of a $\mathrm{pH}$ sensitive dye, 9-amino-6-chloro-2-methoxyacridine (ACMA), indicates acidification of the lumen of the proteoliposomes containing CusA when an inwardly directed $\Delta \mathrm{pH}$ was imposed. The same experiment with the CusA D405N variant, which was previously reported to be an inactive variant, also led to ACMA quenching, although at a slightly lower rate. Under application of an inwardly directed $\Delta \mathrm{pH}$ and a $\Delta \psi$ (negative inside), CusA-containing proteoliposomes showed a strong quenching of the incorporated PGSK dye, suggesting strong $\mathrm{Ag}^{+}$influx.

The Major Facilitator Superfamily-(MFS-) type EmrAB-TolC pump has an analogous structural setup as the RND-type AcrAB-TolC pump. To examine the efflux of one of its substrates, carbonyl - cyanide m-chlorophenylhydrazone (CCCP), a plate-based susceptibility assay was used. The presence of the EmrAB-TolC pump confers lower susceptibility levels towards CCCP in E. coli, compared to cells not expressing the pump or cells expressing only the MFS component, indicating that EmrAB-TolC extrudes CCCP.

The work done in this study opens up a path towards investigation of drug and metal resistance in vitro. The methodologies to obtain proteoliposomal samples of multicomponent efflux pumps and subsequent measurements of drug/metal ion and $\mathrm{H}^{+}$fluxes, as well as the determination of pump assembly are crucial for the future research on pump catalysis and 
transport kinetics. The in vivo drug-plate assays done in this work provide initial insights for future investigations of the drug susceptibility of E. coli expressing the MFS-type tripartite efflux pumps. 


\section{Zusammenfassung}

Einer der weltweit größten Sorgen ist die steigende Zahl der Infektionen, die durch multiresistente Bakterienstämme verursacht werden. Jährlich sterben 33,000 (ECDC，2018, https://www.ecdc.europe.eu/) von Menschen aufgrund mangelnder Behandlungsmöglichkeiten und unwirksamen Antibiotika. Pathogene Bakterien können über verschiedene Mechanismen Antibiotikaresistenzen aufbauen. Efflux von Antibiotika aus der Zelle ist einer dieser Mechanismen. Die bakteriellen Effluxpumpen sind für den Export der toxischen Verbindungen aus dem Zytoplasma und Periplasma heraus verantwortlich. Auf diese Weise bleiben die Toxine unter ihrer wirksamen Konzentration in der Zelle und das Bakterium überlebt. In Gram-negative Bakterien sind die komplexen DreigliedrigeEffluxpumpen am effektivsten. Sie durchspannen die gesamte Bakterienhülle und bestehen aus einer inneren Membrankomponente, welches ein Mitglied der RND-Superfamilie („Resistance-Nodulation cell Division“) ist, einem OMF-Protein („Outer Membrane Factor“) und einem MFP-Protein („Membrane Fusion Protein“), welches die innere und äußere Membran-Komponenten zu einer aktiven Effluxpumpe verbindet. Proteine der RNDSuperfamilie in Gram-negativen Bakterien spielen eine wichtige Rolle im bakteriellen Kampf gegen Antibiotika. Die meisten RND-Mitglieder sind Antibiotika-Transporter, einige Mitglieder exportieren aber auch Schwermetall-Ionen. Heute ist viel über die Strukturen der einzelnen Komponenten und der dreigliedrigen Gesamtstrukturen dieser Pumpen bekannt, aber es gibt immer noch unbeantwortete Fragen: Welche Faktoren hemmen oder begünstigen die Komplexbildung? Was sind die kinetischen Parameter des Substrattransports? Wie verhält sich die Pumpe in Gegenwart verschiedener Inhibitoren? Wie sieht der Substrattransportweg aus? Welche Möglichkeiten gibt es, diese Pumpen in vitro zu untersuchen? In der vorliegenden Arbeit werden einige dieser Fragen angegangen. Einige der Effluxpumpen des klinisch relevanten Bakteriums E. coli wurden isoliert und in Membran-rekonstituierten Systemen funktionell untersucht.

Die in dieser Studie analysierten Transporterkomplexe AcrAB-TolC und EmrAB-TolC sind Mitglieder der RND- bzw. MFS-Superfamilien und transportieren Antibiotika aus der Zelle im Austausch gegen Protonen über die innere Membran. Ebenfalls Teil dieser Arbeit ist CusCBA, ein $\mathrm{H}^{+} /$Schwermetall Antiporter der RND-Superfamilie. Die P-Typ ATPase CopA ist eine ATP-Hydrolyse angetriebene Schwermetall-Effluxpumpe, die in dieser Studie als 
Modell für die Entwicklung eines In-vitro-Assays für die Untersuchung des SilberionenTransports verwendet wurde.

Für die Untersuchung der Effluxpumpen - AcrAB-TolC, CopA und CusA wurden die einzelnen Komponenten überexprimiert, aufgereinigt, und in großen unilamellaren Vesikeln (LUVs - „Large Unilamellar Vesicles“) rekonstituiert. Die $\mathrm{H}^{+}$-Transport Aktivität von AcrB in LUVs wurde durch die Verwendung den pH-sensitiven Farbstoffs Pyranin gemessen. Der Farbstoff wurde während der Rekonstitution in die Proteoliposomen inkorporiert. Bei Ansäuerung des Lumens der Liposomen nimmt das Fluoreszenzsignal von Pyranin ab. AcrB benötigt für seine Aktivität einen Protonengradienten. In der Anwesenheit des pH-Gradienten und eines Substrates (Taurocholat), werden Protonen in die Proteoliposomen transportiert und das Fluoreszenzsignal des Farbstoffs wird gequenched. Verwendet man statt dem AcrB Wildtyp eine Variante von AcrB, mit einer Substitution im Protonenrelais-Netzwerk an der Position D407, kann keine Aktivität beobachtet werden. Die Ko-Rekonstitution von AcrA mit AcrB oder die inaktive AcrB_D407N Variante, ergab keine Steigerung des jeweiligen $\mathrm{H}^{+}$ Influx in den Proteoliposomen.

Für die Untersuchung der Komplexbildung von AcrAB-TolC wurde ein in vitro Assay etabliert. Die starke, nicht-kovalente, Streptavidin-Biotin-Bindung wurde genutzt, um gezielt in Liposomen rekonstituierte AcrAB-TolC-Komplexe $\mathrm{zu}$ isolieren. Die beiden Proteoliposomen-Typen (mit AcrAB oder TolC) waren im Wechsel jeweils mit Biotin oder ohne Biotin markiert. Nach erfolgreichem „Pull-Down“ der Proteoliposomen mit Streptavidin-beschichteten magnetischen Kügelchen, wurde die Interaktion der Pumpenkomponenten auf dem SDS-PAA-Gel nachgewiesen. Die Komplexbildung wurde unter verschiedenen Bedingungen detektiert, aber nur wenn die TolC-Proteoliposomen mit Biotin markiert waren, wurde eine deutliche Komplexbildung beobachtet. Auch in der Gegenwart eines Substrats und/oder eines pH-Gradienten über die Liposomenmembran, wurde die Komplexbildung beobachtet. Auch ohne pH-Gradient, konnte in der Anwesenheit von Substrat oder Inhibitor ein AcrAB- und TolC -Komplex nachgewiesen werden. Wenn kein $\mathrm{pH}$-Gradient angelegt wurde und auch kein Substrat oder Inhibitor vorhanden war, war die in vitro-Komplexbildung auch bei längerer Inkubationszeit zwischen den Komponenten noch möglich. 
Diese Studie legt nahe, dass AcrAB- und TolC-Proteoliposomen in vitro miteinander interagieren können, wodurch sich eine Vielzahl von Anwendungsmöglichkeiten und Assays ableiten lassen, die sich auf den Substrattransport konzentrieren.

Seit Jahren wird kontrovers diskutiert, ob RND- und OMP-Komponenten ohne MFP miteinander interagieren können. In dieser Arbeit wurde beobachtet, dass AcrB mit TolC interagieren kann, da beide Komponenten anschließend auf dem Gel nachgewiesen wurden. Dies sollte in Folgestudien weiter untersucht und bestätigt werden, da die Ergebnisse andeuten, dass diese beiden Komponenten miteinander interagieren können, obwohl sie keinen aktiven Komplex bilden. Der Pull-Down-Assay sollte auch mit z.B. CopAProteoliposomen, die bekanntermaßen nicht mit TolC interagieren, als Negativkontrolle getestet werden.

Um zu zeigen, dass der TolC-Wildtyp geschlossen bzw. inaktiv ist und keine Verbindungen transportiert, wurde TolC YFRS, eine immer offene Mutante, verwendet. TolC YFRS verhält sich wie eine Pore, durch die Pyranin das Lumen der LUVs verlassen kann, was zu einer Abnahme des Fluoreszenzsignals führt. Dies wird nicht beobachtet, wenn der Wildtyp TolC verwendet wurde, was die geschlossene Konformation dieser Komponente der Pumpe bestätigt.

Die Schwermetall-Effluxpumpe CopA wurde als „Proof-of-Principle“ für einen In-vitroAssay zur Beobachtung des Silbertransports in LUVs verwendet. Zunächst wurde bestätigt, ob das produzierte und aufgereinigte Protein in den Detergenzmizellen aktiv ist. Hierfür wurde ein NADH-gekoppelter Assay verwendet. Für dieses Assay wird die Absorption bei $340 \mathrm{~nm}$ gemessen. Diese nimmt ab, wenn NADH oxidiert wird. Dies geschieht proportional zur ATP-Hydrolyse. Das Quenchen der Fluoreszenz des im CopA-Proteoliposomen vorhandenen metall-sensitiven Farbstoffs Phen Green SK (PGSK) in Anwesenheit von ATP und $\mathrm{Ag}^{+}$wird beobachtet. Der Farbstoff kann Mono- und Divalente Metallionen binden, was die Fluoreszenz des Farbstoffs quencht. Wenn keine Silber-Ionen vorhanden waren wurde ein Quenching beobachtet, das nach 300 Sekunden 80\% weniger Quenching aufwies im Vergleich zum Experiment in Anwesenheit von $\mathrm{Ag}^{+}$Ionen. Wenn die Liposomen proteinfrei sind, wird kein Quenching beobachtet. Durch die Anwesenheit von Natriumazid, wird eine geringe Minderung der Proteinaktivität beobachtet. Dies bestätigt, dass Natriumazid kein Inhibitor der P-Typ-ATPase CopA ist. 
Die Untersuchungen an der zur RND-Superfamilie gehörenden SchwermetallionEffluxpumpe CusA aus E. coli konzentrierten sich auf die Identifizierung des Substrattransportwegs durch die Pumpe. Frühere Studien zeigten, dass CusA Silberionen direkt aus dem Zytoplasma aufnimmt und transportiert (Long et al., 2010). Es wurde vermutet, dass der Transport in vivo auch aus dem Periplasma erfolgen kann, wie es bei anderen RND-Mitgliedern (z. B. AcrB) der Fall ist. Das Vorhandensein des Substrats außerhalb des Proteoliposoms würde das „Right-side-Out“ orientierte CusA dazu veranlassen Substrate, die im Außenmedium vorhanden sind zu transportieren und somit das Periplasma imitieren. Wenn der äußere Puffer sauer ist, würden Protonen ins Innere der CusAProteoliposomen transportiert. Das Kontrolexperiment mit einer mutmaßlichen inaktiven CusA Variante, D405N, zeigte auch Protonen-Influx, allerdings mit einer etwas niedrigen Rate. Der Protonen-Influx wird mit dem pH-sensitiven Farbstoff ACMA nachgewiesen, der im Inneren des Liposoms durch Bindung von $\mathrm{H}^{+}$akkumuliert wird, was zu einer FluoreszenzQuenching führt. Wenn ein $\Delta \mathrm{pH}$ and a $\Delta \psi$ (Innen negativ) über die Membran angelegt wird, wird ein starker Silberionentransport, abgeleitet vom Quenching des Fluoreszenz-Farbstoffes PGSK beobachtet. Die Messungen, die mit ACMA und PGSK in der gleichen Anordnung durchgeführt wurden, wiesen darauf hin, dass die CusA Proteine in der „Inside-Out“ Orientierung eine Aktivität aufweisen. Die spezifischen Transportwege können jedoch nur durch weitere Experimente eindeutig festgelegt werden.

Die Anfälligkeit von E. coli in Gegenwart verschiedener Toxine wurde in vivo bestimmt. Diese Arbeit zeigt die geringere Anfälligkeit der Zellen gegenüber CCCP, wenn die Effluxpumpe EmrAB-TolC überexprimiert wird. Die Anfälligkeit der Zellen verändert sich nicht gegenüber dem Wildtyp E. coli, wenn nur die Major Facilitator Superfamily(MFS)Komponente EmrB überexprimiert wird. Zellen, die eine $a c r A B$ Deletion im Chromosom tragen zeigen eine höhere Anfälligkeit gegenüber diesem Toxin, was auf die Rolle der AcrAB-Pumpe bei der Extrusion dieser Verbindung hinweist.

Diese Studie behandelt ebenfalls die Frage nach der Qualität der Proben nach erfolgreicher Rekonstitution durch die Bestimmung der Rekonstitutionseffizienz. Auch wenn die Rekonstitutionseffizienz nicht ausschließlich für die Gesamtqualität der Probe verantwortlich ist, so spielt der Rekonstitutionsprozess dennoch eine ausschlaggebende Rolle. Es ist zu beachten, dass das Protein aus der natürlichen Umgebung entnommen und in eine künstliche Membran eingebracht wird. Die Bedingungen, denen das Protein hier ausgesetzt ist, beeinflussen die Stabilität und die Aktivität der Proteoliposomen und damit der 
Probenqualität. Daher ist zu empfehlen, dass das Design des Rekonstitutionsprozesses auf das entsprechende in vitro Assays abgestimmt und optimiert werden sollte, um die bestmögliche Rekonstitutionsbedingungen für das Inkorporieren des Zielproteins in die neue Lipidmembran zu schaffen.

Diese Studie liefert die Grundlage für zukünftige Studien von MehrkomponentenEffluxpumpen. In vitro Assays, die für die Charakterisierung von AcrAB-TolC, CusA sowie CopA verwendet wurden, liefern eine Grundlage für das weitere Studium des ToxinTransports in Gram-negativen Bakterien (Transport von Antibiotika, Farbstoffen, Schwermetall-Ionen, etc.). Dies wird durch die Untersuchungen der Komplexbildung unterstützt, die in zukünftigen Studien auf die Untersuchungen der AssemblierungDisassemblierungs-Kinetik der Effluxpumpen-Komponenten erweitert werden kann. Die Verwendung von Fluoreszenzfarbstoffen als Reportersystem hat sich als nützlich und reproduzierbar erwiesen, kann aber durch die Verwendung verschiedener Maschinen (z.B. Stop-Flow) für die Detektion verbessert werden. Dies könnte bei der Überwachung schneller Prozesse und schneller Transporte helfen, um die Kinetik der Systeme zu bestimmen. Diese Studie schlägt auch die Verwendung von radioaktiv markierten Substraten für die Beobachtung des Substrattransports in vitro vor, da radioaktive Komponenten mit deutlich höherer Sensitivität detektiert werden können.

In vivo-Studien, die hier begonnen wurden, können mit Untersuchungen der Mutante der EmrAB-TolC-Effluxpumpe fortgesetzt werden. Varianten, die eine Mutation im Protonenrelais-Netzwerk und/oder in den spezifischen Bindungsstellen tragen, sollten für die genauere Bestimmung des Transports, der Kinetik und der Affinität der Substrate verwendet werden. Dies erstreckt sich auch auf die Bestimmung des Verhaltens der aktuell bekannten Inhibitoren, was einen enormen Vorteil beim Design neuer potenter Inhibitoren darstellt.

Diese Studie leistet einen elementaren Beitrag zum Verständnis über den Mechanismus, mit dem Gram-negative Bakterien Medikamenten- und Metallresistenz erwerben. Qualitativ hochwertige Proteoliposomen-Proben und Etablierung von Assays zur Analyse des Multikomponenten-Efflux-Pumpen-Assembly sind die Basis für zukünftige Arbeiten. Erste Studien, die in in-vivo-Experimenten mit der MFS-Pumpe, EmrAB-TolC, durchgeführt wurden, geben Aufschluss über die Empfindlichkeitsstufen und Stämme, die in zukünftigen Studien zu dieser Pumpe verwendet werden sollten. Diese Arbeit präsentiert grundelegende 
Fakten, die für die funktionellen Untersuchungen von bakteriellen Membranproteinen erforderlich sind.

Bakterien finden einen Weg, Toxine auszustoßen, zu modifizieren oder gar nicht erst aufzunehmen, um zu überleben. Dieser Sachverhalt ist für die Bekämpfung von resistenten Mikroben von großer Wichtigkeit. Jede neue Erkenntnis über die Resistenzmechanismen ist ein Schritt vorwärts in der Bekämpfung multiresistenter Bakterienstämme. Diese Arbeit zeigt einen Weg auf, um gerichtet zukünftige Antibiotika und Efflux-Pumpen Inhibitoren auf ihre Potenz hin zu analysieren. 


\section{Chapter I: Introduction}

\subsection{Escherichia coli, a facultative pathogen}

Escherichia coli is a rod-shaped Gram-negative facultative anaerobic bacterium commonly found in the lower intestine of birds and mammals. Most of the E. coli strains are harmless and part of the normal microbiota of the gut. The relationship between E. coli and the host can be described as interdependency, where both, the host and the bacteria, receive benefits from each other without harming one another. The bacterium gets protected by the host; it is present in the host's environment, using host's nutrients, transport and circulation. In return, E. coli prevents colonization of pathogens by inducing colonization resistance in the host, while producing vitamin K (Krogfelt et al., 2004)(Rastegar Lari et al., 1990)(Vollaard \& Clasener, 1994)(Hudault, 2001)(Schamberger et al., 2004).

E. coli was first discovered in 1884 , by German microbiologist and paediatrician Theodor Escherich when he began a study of infant gut microbes and their role in digestion and disease. By the 1940s, E. coli was established as a model organism and later on it was used in the molecular biology revolution in the 1950s (Blount, 2015). Nowadays, for the laboratory work, E. coli is still the first organism of choice.

As any other microorganism, E. coli as well is a serious survivor and warrior, since it has developed mechanisms with which it fights for its life and existence in the world where a lot of molecules were designed just for the purpose of killing it.

\subsubsection{E. coli structure}

For decades, E. coli was used as a model system, since in a very small size $(1.5$ to $5.5 \mu \mathrm{m}$ long and 0.5 to $1.0 \mu \mathrm{m}$ in diameter) it packs its genetic material and the whole metabolic machinery (Nanninga, 1998). It divides every 20 minutes in a laboratory under aerobic, nutrient-rich conditions. 


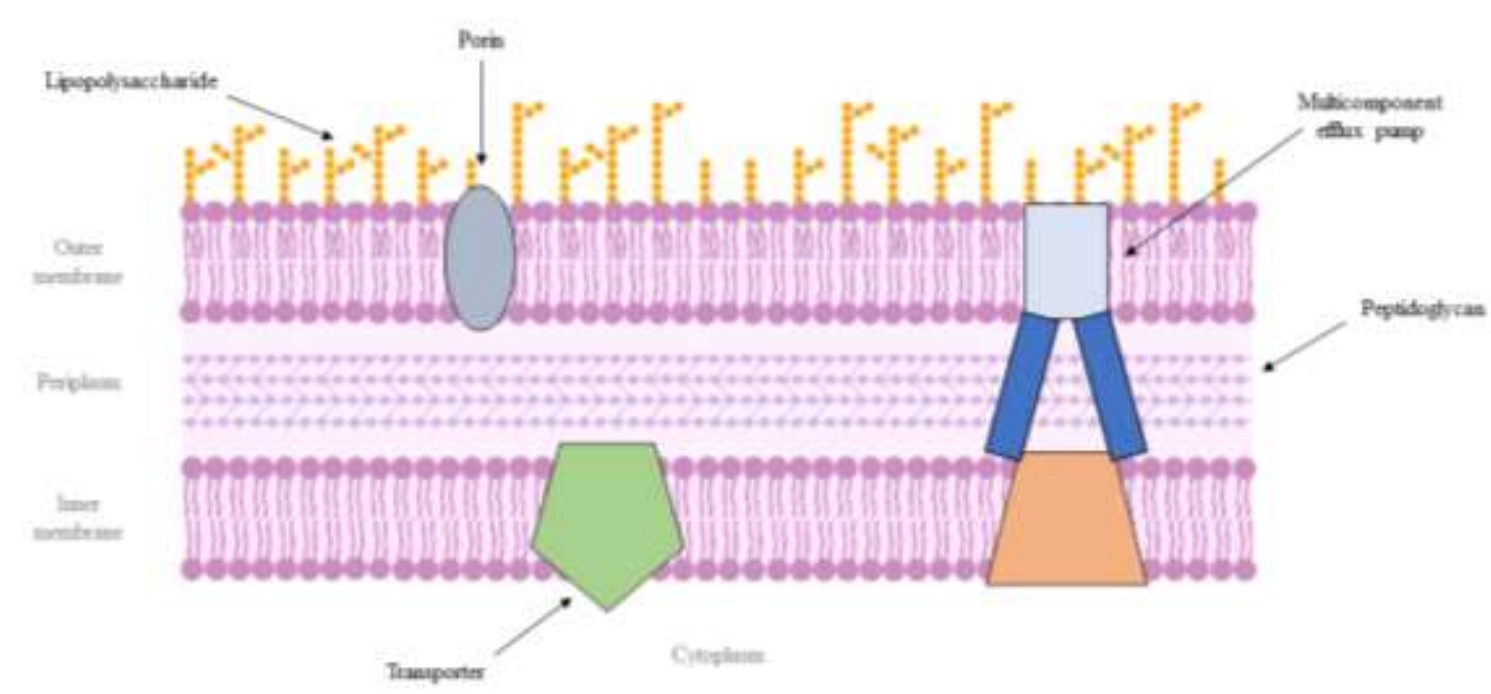

Figure 1. Schematic representation of the structure of the cell envelope of E. coli. The envelope consists of an outer and an inner membrane that encapsulate the periplasm, and separate the cytoplasm of the cell from its environment. The shape of the cell is defined by the peptidoglycan layer, positioned in the periplasm. Membrane proteins (e.g., porins, transporters) are embedded in the inner and outer membrane. Some membrane proteins can form complexes that span the bacterial envelope (multicomponent efflux pumps). The outer membrane has lipopolysaccharides that are part of the outer leaflet in most Gramnegative bacteria. Figure was made with help of BioRender (https://app.biorender.com/).

The envelope of rod-shaped E. coli has three layers: the cytoplasmic membrane, the peptidoglycan (murein) layer and the outer membrane (Figure 1). The peptidoglycan is embedded into the periplasm, a compartment between inner and outer membrane. The peptidoglycan layer is made of linear glycan strands cross-linked by short peptides. This layer defines the shape of the bacterial cell. The outer membrane of Gram-negative bacteria is the most asymmetric membrane described so far. The inner leaflet is composed of phospholipids, while the outer leaflet is built from glycolipids. For most Gram-negative bacteria the glycolipids are lipopolysaccharides (LPS), for a few species they are glycosphingolipids. It has been modelled with the asymmetric membrane with LPS being the main component in the bacterial outer leaflet and a phospholipid mixture (PL) as inner leaflet. It has to be mentioned, that unlike eukaryotic membranes, the bacterial membrane has a much higher degree of flexibility, which comes from the packing density of the LPS in the outer leaflet (Paulowski et al., 2020). 


\subsubsection{Pathogenic E. coli}

"Colibacillosis" is a term used for a disease caused by E. coli, which resides in the lower intestines of most warm-blooded mammals (Poirel et al., 2018). As much as E. coli is providing a healthy gastrointestinal tract, this microorganism can also be a cause of diarrheal diseases and is even responsible for colitis-associated colorectal cancer (CRC) (Arthur et al., 2012).

Diarrheal illnesses are severe death threat and remain the second leading cause of death in children younger than 5 years globally and nearly one in every five child deaths is due to this illness (Wardlaw et al., 2010). Diarrheal diseases are being responsible for about 750,000 of a total of 4.3 million deaths of African children up to four years of age (Bos et al., 2006). Enterotoxigenic E. coli (ETEC), is a group of E. coli that undergoes two-step pathogenic process: intestinal colonization, followed by elaboration of diarrheagenic enterotoxin(s) (Evans \& Evans, 1996).

Colibactin is a genotoxin that induces DNA double-strand breaks that have been suggested to lead to carcinogenesis. Colibactin is produced by $E$. coli strains harbouring the $p k s$ island that codes this polyketide-peptide genotoxin (Oliero et al., 2021). One of the E. coli strains connected with CRC is E. coli NC101 (Arthur \& Jobin, 2013).

The most dangerous strain of $E$. coli is "the infamous" $\mathrm{O} 157: \mathrm{H} 7$, an enterohemorrhagic shiga-toxin producer. This toxin attacks small blood vessels, killing intestinal cells and causing bloody diarrhoea uremic syndrome (HUS), which can be a deadly condition in the sensitive adults and infants (Griffin \& Tauxe, 1991). Especially difficult is that this condition cannot be treated with antibiotics, since they increase the risk of HUS (Smith et al., 2012).

E. coli strains that live outside the intestinal, extraintestinal pathogenic E. coli can cause very severe health problems. These E. coli strains can cause bacteremia, sepsis, neonatal meningitis and neonatal sepsis, where the later very often leads to death. Unfortunately, E. coli is present in intraabdominal infections and nosocomial pneumonia as well as in osteomyelitis, cellulitis, wound infections and in severe urinary infections (J. T. Poolman \& Wacker, 2016)(de Louvois, 1994). The severe nature of extraintestinal pathogenic E. coli strains cannot be dismissed but it is overlooked since it does not cause the global epidemic fashion as the E. coli strain O157:H7 does (Johnson \& Russo, 2002). 


\subsection{Antibiotics and antibiotic resistance}

Antimicrobial resistance is a global, tremendous and serious threat, that increases the risk of disease spreading and higher number of deaths caused by microbes. Nowadays, it is enormously important to understand how the resistance and survival mechanisms work in bacteria, viruses, fungi and parasites, since only the bigger understanding gives us more possibilities to ensure existence of the human race on this planet. As much as this sounds light years away, we are now, at this moment, suffering from a virus attack (SARS-CoV-2), which combined with bacterially induced inflammations, causes vary rapid, sever and lethal situations. That said, the emergence, spread and persistence of multidrug-resistant (MDR) bacteria or "superbugs" are one of the most important concerns not only for the scientific world but also on a global scale. MDR bacteria are present in animals and humans alike. Due to ever closer contact between animals and humans, bacteria can spread easily across species.

The antibiotic resistance crisis has been linked to the overuse and misuse of antibiotics. The lack of new drugs coming to the market comes from the reduced economic incentives and tighter regulations for the production.

It is intimidating when seeing the numbers: every year, 700,000 people die from infections caused by MDR bacteria (Dadgostar, 2019). The estimation by the Wellcome Trust and the British Government is that 10 million people will die during the year 2050 as a result of infections by antimicrobial resistant bacteria, which would then exceed the 8.2 million cancer deaths in 2019 (Wall, 2019)(Bassetti et al., 2017)(de Kraker et al., 2016). 


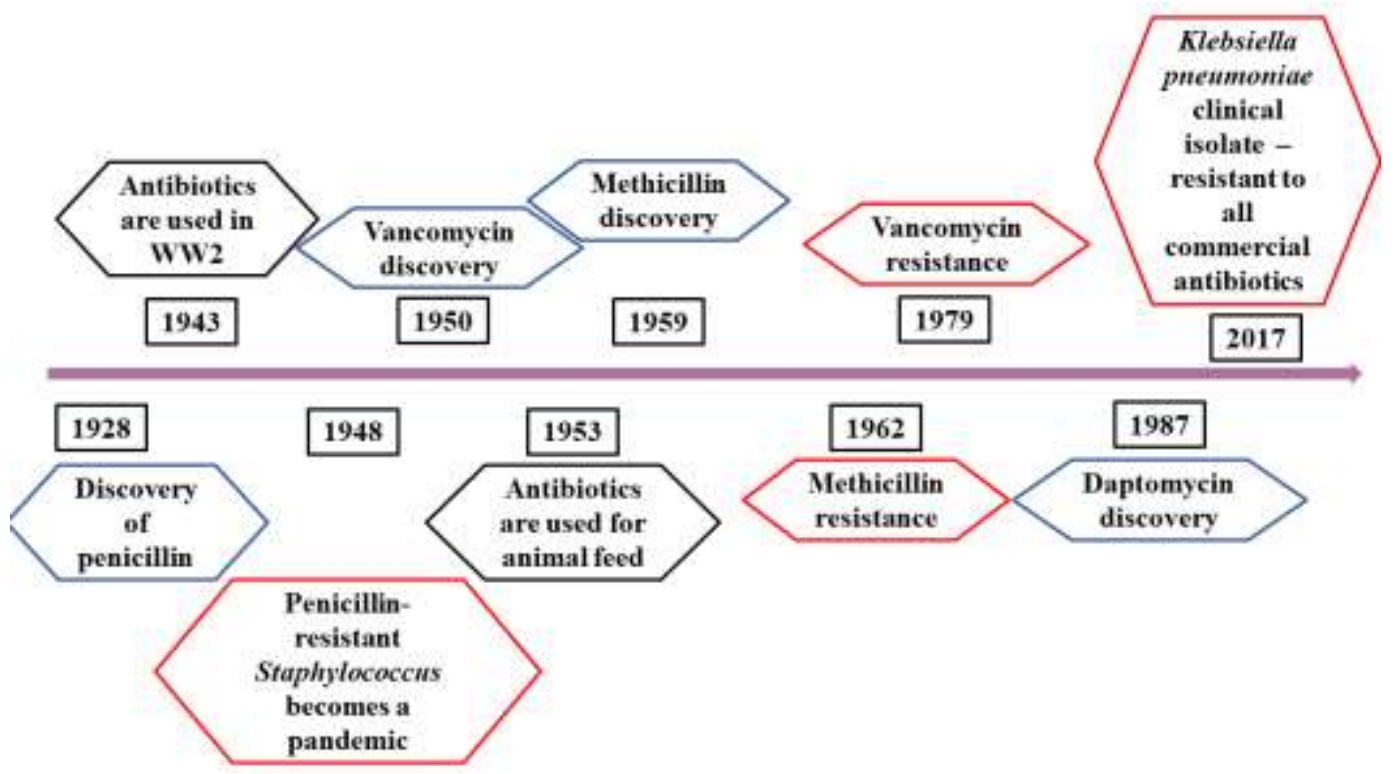

Figure 2. Schematic representations of the introduction of antibiotics on the market and appearance of their resistance. This is a fraction of all the antibiotics that have been developed. Blue shapes represent the event of antibiotic discoveries. Red shapes represent resistance development events. Black shapes depict historical events that mark the beginning of antibiotic success story that ultimately led to misuse (WW2- World War 2).

Since the first antibiotic was discovered in 1928 by Sir Alexander Fleming, many other antibiotic discoveries followed. Antibiotics were first prescribed in the 1940s. Mass use of penicillin was successfully implemented during the Second World War, which then backfired in the clinical problem of penicillin resistance. Even though this problem was overcome shortly after with the development of new antibiotics, the first case of methicillin-resistant Staphylococcus aureus (MRSA) was identified across the world, during 1960s (Figure 2) (Sengupta et al., 2013)(Ventola, 2015).

\subsubsection{Antibiotics and their targets - mechanism of action}

Antibacterial agents can be divided into several groups, according to the following criteria:

1) type of action, 2) source, 3) spectrum of activity, 4) chemical structure and 5) function.

1) According to the type of action, antibiotics can be described as bacteriostatic and bactericidal. The definition of bacteriostatic is that the agent prevents the growth of bacteria, while bactericidal means that it kills bacteria. However, the in vitro microbiological determination of whether the agent used is bactericidal or bacteriostatic might be dependent on 
growth conditions, bacterial density, test duration and extent of reduction in bacterial numbers. Better is to describe most antibacterials as potentially being both bactericidal and bacteriostatic (Pankey \& Sabath, 2004).

2) When talking about source, antibiotics can be from fungal sources, semi-synthetic or completely synthetic. Semi-synthetic products are obtained from natural products and altered chemically (e.g., the alteration helps in the increase of the effectiveness of the synthesised product).

3) Antibiotics can be with narrow or broad spectrum of activity. Narrow-spectrum antibiotics target a few types of bacteria (e.g., older penicillins (penG) or macrolides) while broadspectrum antibiotics (e.g., aminoglycosides, quinolones) target many types of bacteria.

4) According to their chemical structure, antibiotics can be divided into e.g., $\beta$-lactams, aminoglycosides, macrolides, quinolones and fluoroquinolones, nitroimidazoles, tetracyclines, sulphonamides or streptogramin antibiotics (Adzitey, 2015)(Ullah \& Ali, 2017).

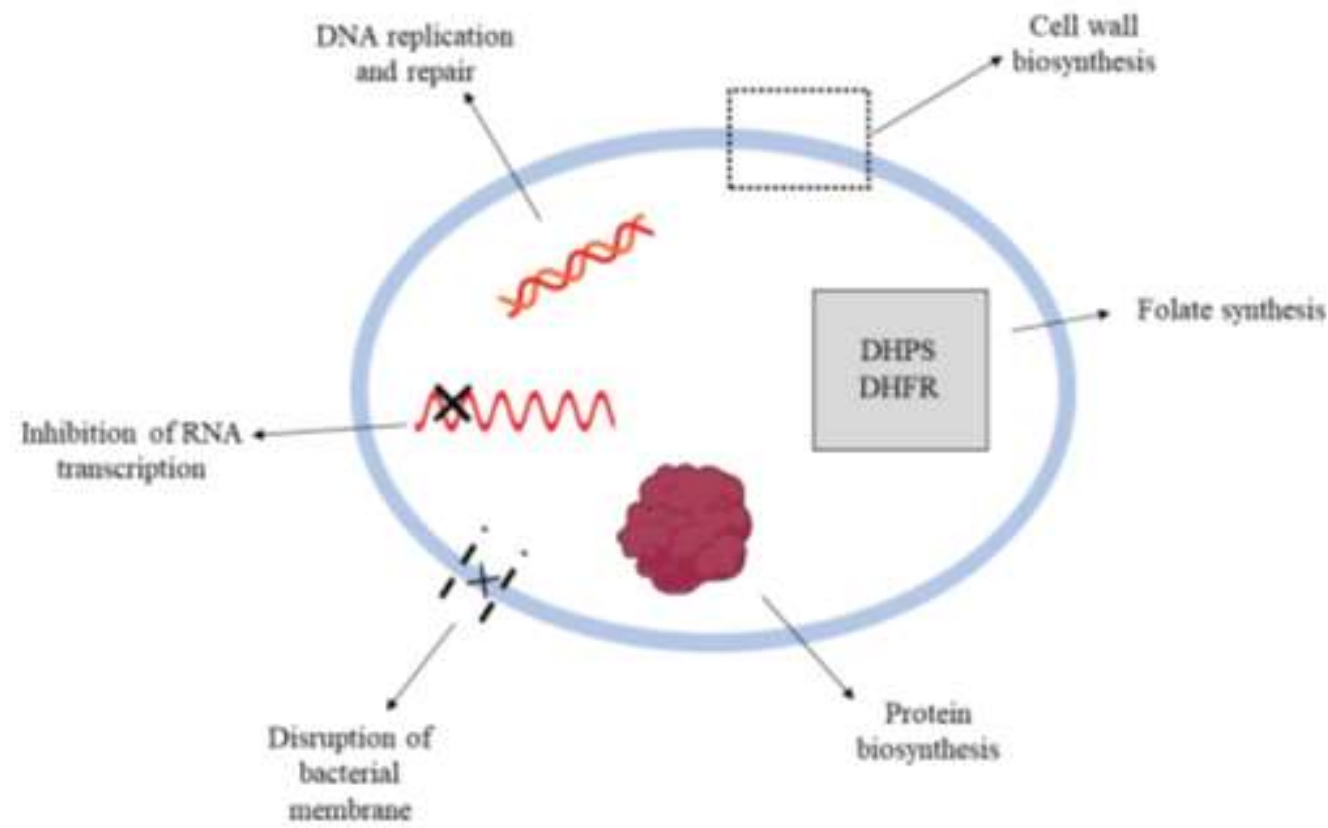

Figure 3. Schematic representation of drug targets in the bacterial cell. The bacterial cell structures that are targeted by the drugs are indicated. Figure was made with help of BioRender (https://app.biorender.com/). 
5) According to the function (or their target) antibiotics can be divided into: a) cell wall synthesis inhibitors, b) disruptors of bacterial membrane, c) protein synthesis inhibitors, d) inhibitors of DNA or RNA synthesis, and e) inhibitors of essential precursor synthesis (folate biosynthetic pathway).

a) One of the most used ways to stop the growth of the bacteria is to stop cell wall synthesis by inhibiting the synthesis of the peptidoglycan layer of bacterial cell walls. $\beta$-lactams (characterized by a chemical structure called a $\beta$-lactam ring) are mostly used for these purposes. The final step in the cell wall biosynthesis is facilitated by penicillin-binding proteins (D-alanyl-D-alanyl transpeptidases). $\beta$-lactams work as an equivalent molecule to Dalanyl-D-alanyl substrate and they inhibit the transpeptidase reaction and block the synthesis of the peptidoglycan layer. In addition to penicillin and cephalosporins, vancomycin from a class of glycopeptide antibiotics also targets the peptidoglycan, by targeting the peptide substrate and preventing it from reacting with either the transpeptidases or the transglucosylases. The effect is the same - failure to make peptidoglycan crosslinks, which at the end results in changes in cell shape and size and ultimately cell lysis. $\beta$-lactams and vancomycin show synergy when used in combination (Walsh, 2000)(Ullah \& Ali, 2017).

b) Daptomycin interacts with calcium ions in the cytoplasmic membrane of Gram-positive bacteria and when it inserts itself into the membrane, it causes depolarization of the membrane potential and loss of intracellular content (Straus \& Hancock, 2006).

c) Different classes of antibiotics are involved in the inhibition of the protein synthesis: macrolides, aminoglycosides, oxazolidinones, as well as tetracyclines and phenicols. This is not a surprise since a large machinery is involved in the translation of RNA into proteins. The bacterial ribosome is made of three ribosomal RNAs (rRNAs; 16S, 23S and 5S) and $\sim 54$ ribosomal proteins. Protein synthesis can be divided into four main steps: initiation, elongation, termination and recycling. Each step of the protein synthesis can be targeted by antibacterial drugs. The first targets are: the $30 \mathrm{~S}$ initiation complex, the formation of the $70 \mathrm{~S}$ ribosome by the $30 \mathrm{~S}$ initiation complex and the 50S ribosome, and the elongation process, assembly of amino acids into a polypeptide. However, even the final step of recycling for the next initiation is being inhibited if fusidic acid is being present (Wilson, 2014)(Arenz \& Wilson, 2016).

In more detail, the initiation of protein synthesis is inhibited by the antibiotics edeine, kasugamycin, pactamycin and thermorubin on the $30 \mathrm{~S}$ subunit, and by orthosomycins 
avilamycin and evernimicin as well as thiostrepton on the 50S subunit. The elongation cycle is inhibited by streptomycin, tetracyclines and glycylcyclines. Peptide-bond formation is inhibited by blasticidin S, chloramphenicol, lincosamides, oxazolidinones, pleuromutilins, puromycin, streptogramin A and sparsomycin. Translocation of the tRNAs inhibited by the tuberactinomycins capreomycin and viomycin, aminoglycosides hygromycin B, neomycin and paromomycin, as well as fusidic acid, spectinomycin and thiostrepton. Elongation of the nascent chain is inhibited by macrolides (erythromycin), streptogramin B and ketolides (telithromycin). The final phases of termination and recycling are inhibited by peptidyltransferase inhibitors, such as blasticidin S, cloramphenicol, puromycin and sparsomycin, whereas recycling is inhibited by translocation inhibitors, especially fusidic acid (Wilson, 2014).

d) Another important class of inhibitors is the nucleic acid inhibitor class, that can be divided into RNA synthesis inhibitors and DNA replication inhibitors. RNA synthesis inhibitors deal with the transcription process in which mRNA transcripts of genetic material are produced for later translation into proteins. Members of the rifamycin family bind to DNA-dependent RNA polymerase and inhibit the elongation of RNA (by binding to the $\beta$-subunit of the RNA polymerase during transcription). Quinolones work as DNA synthesis inhibitors. They target two essential type II topoisomerases, DNA gyrase and topoisomerase IV. Inhibition of the topoisomerases results in an excess of positively supercoiled DNA and, due to the topoisomerase mechanism of action, leads to DNA strand breakage. This prevents DNA replication (Walsh, 2000)(Fàbrega et al., 2009)(Shen, 1993).

e) The sulphonamide family (Walzer et al., 1988) and diaminopyrimidines (Songsungthong et al., 2021), often in combination, inhibit the folate synthesis, which involves two enzymes in bacteria, dihydropteroate synthase (DHPS) and dihydrofolate reductase (DHFR). 


\subsection{Antibiotic resistance mechanisms in bacteria}

Bacteria can have intrinsic resistance to certain antibiotics. The intrinsic resistance to a particular antibiotic comes from the ability to resist the action of the antibiotic as a result of inherent structural or functional characteristics of the bacterium. Resistance can also be acquired via mutations in chromosomal genes and by horizontal gene transfer (Blair et al., 2015).

Three main mechanisms of antibiotic resistance are: 1) prevention of antibiotic to reach the target, 2) changes in antibiotic target and 3) direct modification of the antibiotic (Blair et al., 2015).

1) To prevent antibiotics to reach their target in Gram-negative bacteria, the permeability of the outer membrane $(\mathrm{OM})$ can be reduced (Figure 4). The OM is a great permeability barrier, but allows passage of water, ions and small, hydrophilic molecules through narrow porins. Members of the ESKAPE pathogens (Enterococcus faecium, Staphylococcus aureus, Klebsiella pneumoniae, Acinetobacter baumannii, Pseudomonas aeruginosa and Enterobacter species), that are of special concern when it comes to antibiotic resistance, are Pseudomonas and Acinetobacter, since they show multidrug resistance phenotype because their OM has an exceptionally low permeability due to the mostly closed porin channels (Li \& Nikaido, 2009)(Sugawara et al., 2006). With this, they limit the number of molecules that can cross the OM. Another mechanism to prevent the antibiotic to reach the target occurs via efflux across the inner and outer membrane, mediated by bacterial efflux pumps (Blair et al., 2015).

2) A clever way of protecting themselves, bacteria developed preventive measures against efficient antibiotic binding via changing the structure of the antibiotic target (Figure 4). This can be performed via alteration of the antibiotic target by genetic mutation which can be obtained via acquisition of a gene homologous to the original target or a single point mutation in the gene encoding an antibiotic target. The alteration of the antibiotic target can happen via post-synthesis modification as well. An example for post-transcriptional modification is the erythromycin ribosome methylase ( $\mathrm{erm}$ ) family of genes, which encode for methylases that methylate 16S rRNA and alter the drug-binding site, thus preventing the binding of drug, specifically the binding of macrolides. Besides macrolides, aminoglycosides are protein synthesis inhibitors and they function by binding to the ribosome. One of the mechanisms of 
resistance to aminoglycosides is modification by methylation of the target ribosome (Blair et al., 2015).

3) If a cell directly modifies the antibiotic, it can inactivate it via hydrolysis or by addition of chemical groups (Figure 4). Bacterial enzymes can modify the vulnerable sites of antibiotics with acyl, phosphate, nucleotidyl or ribitoyl groups, thus creating steric hindrances. Several classes of antibiotics are known to be enzymatically modified: $\beta$-lactams, aminoglycosides and macrolides. The enzymes that are responsible form a large and diverse family of antibiotic-resistance enzymes. For example, aminoglycosides are phosphorylated by aminoglycoside phosphoryl transferases, but they can be acetylated by aminoglycoside acetyltransferases, or adenylated by aminoglycoside adenyl transferases (Nikaido, 2009) (Blair et al., 2015).

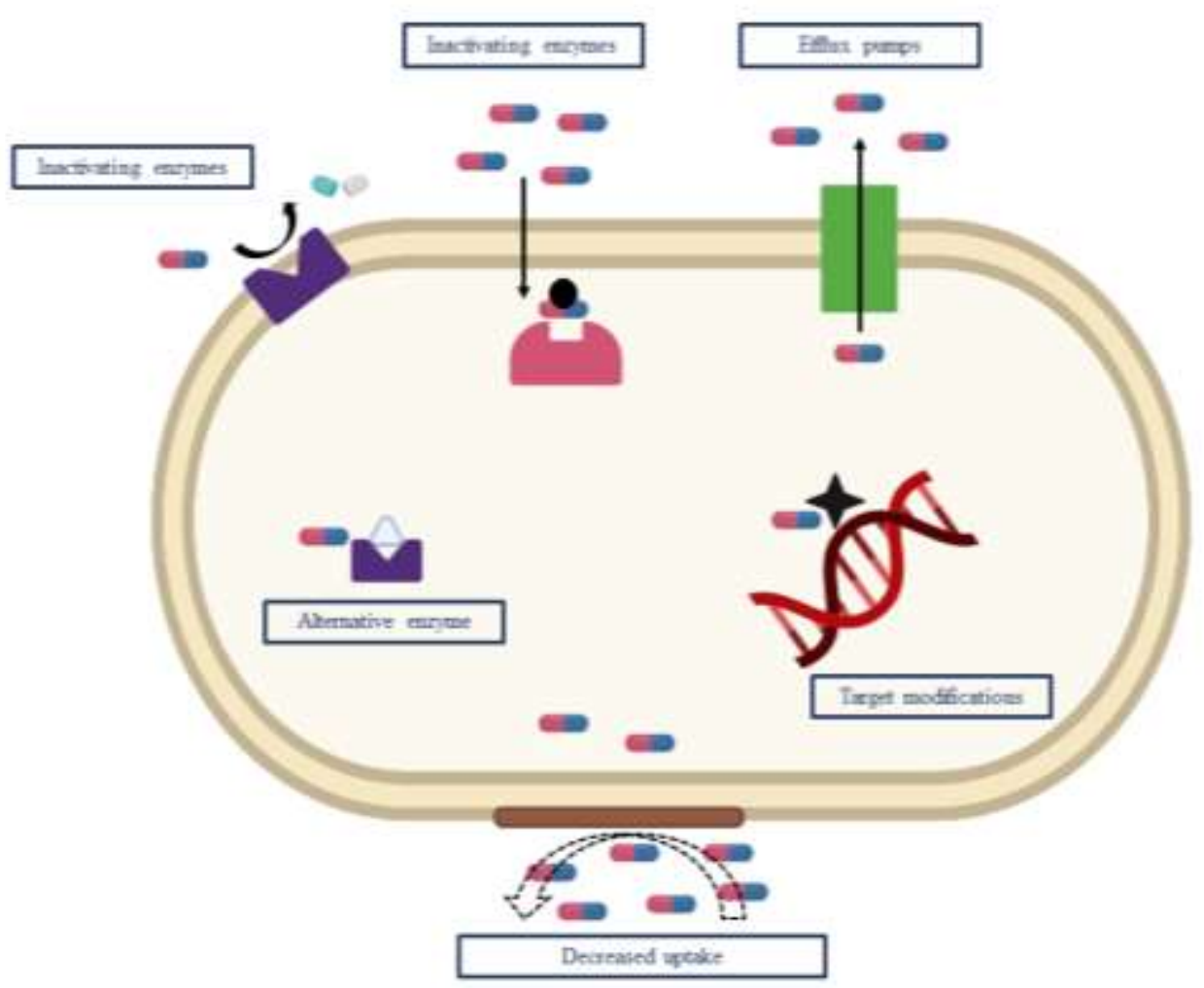

Figure 4. Mechanisms of antibiotic resistance in bacteria. Direct modifications of antibiotics via enzymatic reactions, usage of alternative enzymes, overexpression of the efflux pumps, target modifications or decrease of the permeability of the outer membrane (Gram-negative bacteria) are the ways that organisms use to survive in the environment that contains toxic compounds. Figure was made with help of BioRender (https://app.biorender.com/). 


\subsubsection{E. coli developed resistance to a various number of antibiotic classes}

Antimicrobial resistance in E. coli is thought to be one of the major challenges in both human and animal health. Over time antibiotic resistance has emerged in E. coli against various antibiotic classes. This section, gives an overview over some antibiotic classes and respective resistance to those classes.

$\boldsymbol{\beta}$-lactams. E. coli found in humans and animals developed resistance towards $\beta$-lactams due to specific genes that code for extended-spectrum $\beta$-lactamases (ESBLs) or by overexpressing cephalosporinases (AmpCs)(Poirel et al., 2018). ESBLs are causing the downsized treatment success and limit the possible choices of medications to use for doctors, especially veterinarians, since there is an increasing number of $\beta$-lactam resistant $E$. coli infections in food-producing and companion animals worldwide (Ewers et al., 2012).

Four classes of $\beta$-lactamases have been identified (Nikaido, 2009).

1) Class A enzymes hydrolyse ampicillin and penicillin. It includes: TEM and SHV enzymes, which are also called ESBL (extended spectrum $\beta$-lactamases).

2) Class B $\beta$-lactamases enzymes are used against carbapenems. This class includes metalloenzymes (e.g., NDM-1 (New Delhi Metallo- $\beta$-lactamase)).

3) Class C $\beta$-lactamases represents chromosomally coded enzymes (e.g., AmpC). Class A and $\mathrm{C}$ enzymes are commonly present in Gram-negative bacteria. These enzymes use a similar molecular mechanism as serine- and cysteine- proteases use for hydrolysis.

4) Class $D \quad \beta$-lactamases include a number of enzymes that have been identified in Acinetobacter spp., notably in Acinetobacter baumanii. The members of the oxacillinase (OXA) group are the vast majority of the class D members.

TEM- (named after a patient- Temoniera, from which the $\beta$-lactam-resistant bacteria were isolated) and SHV- (sulphydryl-variable) ESBLs are members of class A of $\beta$-lactamases that were dominant until 2000s and then CTX-M-ESBLs were described (CTX as its acronym, coming from a fact that it has hydrolytic activity against cefotaxime, -M from Munich) (Cantón et al., 2012)(Rawat \& Nair, 2010). Another class of enzymes that acquired resistance 
is the class $\mathrm{C} \beta$-lactamases, also known as AmpC-type, for which the resistance is chromosomally mediated. In E. coli, the $\operatorname{ampC}$ gene is expressed at low levels and is regulated by a growth rate-dependent attenuation mechanism, not by induction, since the $a m p R$ gene is missing in the $\operatorname{ampC}$ region (AmpR is a transcriptional activator that binds to DNA immediately upstream of the ampC promotor) (Honoré et al., 1986)(Jaurin et al., 1982)(Philippon et al., 2002). Carbapenemases started to bring some concerns recently, when the first carbapenemase determinant was identified. The first report on the presence of a carbapenemase encoding gene in E. coli was isolated from a livestock pig farm in Germany. This clinical E. coli isolate (R178) was detected to produce VIM-1 carbapenemase (Fischer et al., 2012).

Quinolones and fluoroquinolones. Quinolones and fluoroquinolones are an important class of antibiotics that is used as bactericidal against virtually all bacteria. Resistance towards these antibiotics comes from mutations in the drug target, the genes for DNA gyrase and topoisomerase IV, as well as reduced permeability of the outer membrane or utilization of efflux pumps (Hooper, 1999)(Hansen et al., 2004)(Yue et al., 2008)(Nikaido, 2003).

Aminoglycosides. Aminoglycosides influence a broad spectrum of bacteria by interfering with the translation. Resistance against aminoglycosides can be acquired by target mutations in 16S RNA and/or S5 and S12 ribosomal proteins. Resistance towards amikacin, tobramycin, gentamicin and netilmicin is developed with the modification of the target site, achieved by methylation of residues G1405 and A1408 of the A site of 16S rRNA (Doi et al., 2016)(Fourmy et al., 1998)(Griffey et al., 1999)(Llano-Sotelo et al., 2009). At the same time, three types of aminoglycoside-modifying enzymes are known: acetyltransferases, nucleotidyltransferases and phosphotransferases (Poirel et al., 2018).

Fosfomycin and tetracyclines. E. coli exhibits resistance towards fosfomycin and a range of tetracyclines. Fosfomycin (Phosphonomycin) is a bactericidal that effects the integrity of the bacterial cell wall (Hendlin et al., 1969). Once in the cytoplasm, fosfomycin acts as an analog of phosphoenolpyruvate (PEP) binding to MurA (UDP-GlcNAc enolpyruvyl transferase) and inactivating the enzyme enolpyruvyl transferase, an essential enzyme in peptidoglycan biosynthesis (Brown et al., 1995). Tetracyclines are a class of broad-spectrum antibiotics. They are used in treatment of a variety of infectious diseases. Tetracyclines inhibit the 30S ribosomal subunit, blocking the binding of the aminoacyl-tRNA to the acceptor site on the mRNA-ribosome complex. As soon as this is done, the cell can no longer function and will 
not be able to grow or further replicate. This characteristic makes the tetracyclines good bacteriostatic (Nemeth et al., 2015). Resistance to tetracycline in bacterial cells is usually mediated by energy-dependent pumping of tetracycline out of the cell (Karami et al., 2006).

Phenicols. Chloramphenicol $(\mathrm{Cm})$ and its fluorinated derivative florfenicol $(\mathrm{Ff})$ are highly potent inhibitors of bacterial biosynthesis of proteins. The resistance in E. coli is mediated by three mechanisms: enzymatic inactivation of nonfluorinated phenicols (cat genes), active efflux of nonfluorinated phenicols ( $\mathrm{cmlA}$ genes) or fluorinated and nonfluorinated phenicols (floR genes), and target site methylation encoded by the multiresistance gene $c f r$, which confers resistance to five classes of antimicrobial agents, including fluorinated and nonfluorinated phenicols (Schwarz et al., 2004).

Sulfonamides and trimethoprim. Resistance to sulfonamides and trimethoprim comes from sul genes encoding dihydropteorate synthetases-insensitive to sulfonamides or $d f r$ genes encoding dihydrofolate reductases-insensitive to trimethoprim, as well as from the modifications deriving from mutations in the genes encoding the target enzymes (van Duijkeren et al., 2018).

Polymyxins. The target of polymyxins is the OM of Gram-negative bacteria. After binding to lipopolysaccharides in the outer membrane, they disrupt both the outer and inner membranes. Because of its hydrophobic tail that is causing membrane damage, it is suggested that polymyxins act in a detergent-like mode of action (Garg et al., 2017). Resistance to polymyxins (colistin) can be acquired in relation with the activity of efflux pumps or due to mutations in chromosomal genes or because of acquired resistance genes. These genes produce modified LPS lipid A, that are less likely to interact with polymyxin (Poirel et al., 2018)(Trimble et al., 2016).

\subsection{Multidrug efflux pumps}

Many bacterial species have armed themselves with mechanisms to fight toxic compounds in nature. Overexpression of efflux pumps is one of the most important mechanisms that leads to intrinsic antibiotic resistance. This process is however, energetically expensive and needs an active metabolic system that will provide for the drug resistance (Zampieri et al., 2017). Efflux pumps reduce the accumulation of the drugs inside the cell. While the drugs are being 
exported through the pumps, bacteria are adapting to the antibiotic and become resistant through other mechanisms (mutations, alterations of the target, etc.) (Li \& Nikaido, 2009). From the first characterisation of the energy driven export of drugs (P-glycoprotein from mammalian cells (Juliano \& Ling, 1976)), the drug export in Gram-negative bacteria has been described as a two-stage process: compound goes from cytoplasm to the periplasm, and from the periplasm out of the cell (Tal \& Schuldiner, 2009).

The (super)families of multidrug transporters are: ATP-Binding Cassette (ABC), Resistance Nodulation cell Division (RND), Major Facilitator (MF), Multidrug and Toxic compound Extrusion (MATE), Small Multidrug Resistance (SMR), and Proteobacterial Antimicrobial Compound efflux (PACE) (super) families (Figure 5).

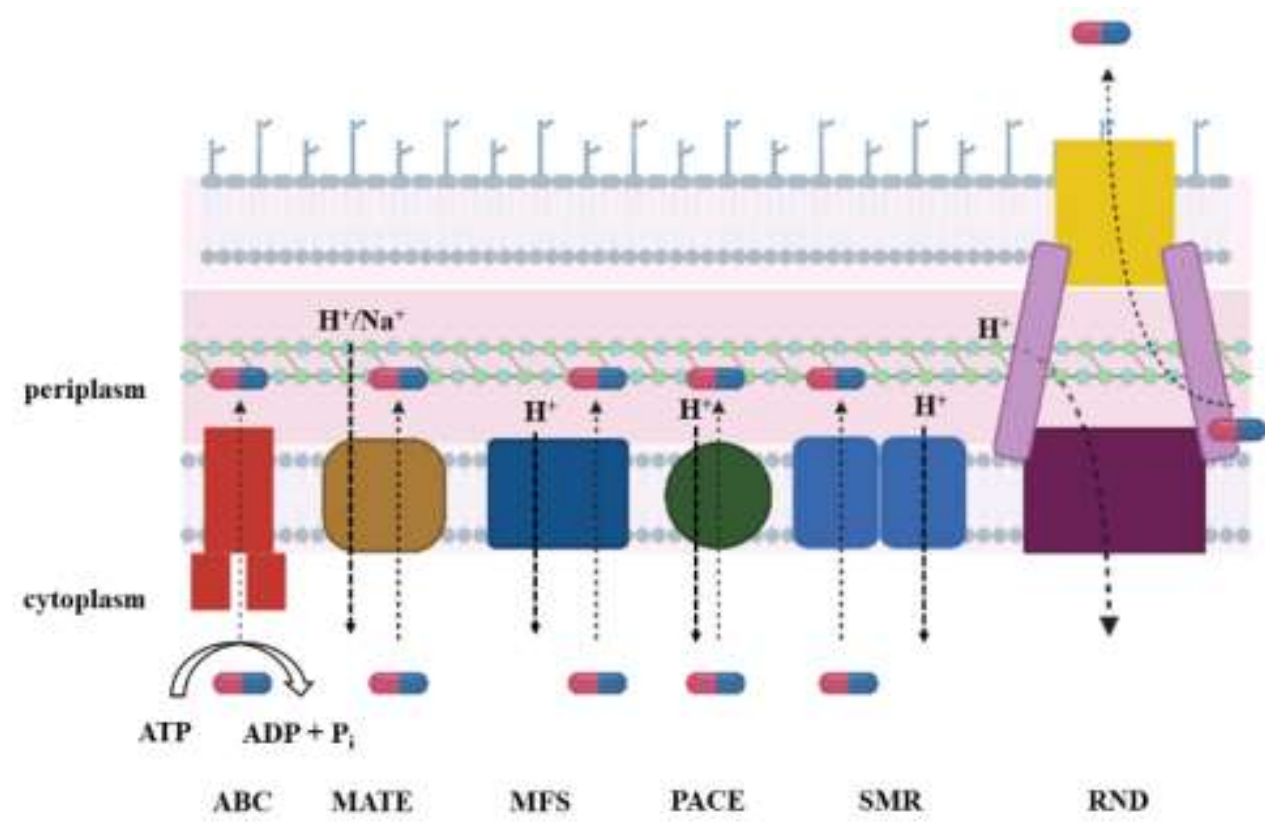

Figure 5. Multidrug efflux pumps in Gram-negative bacteria. Schematic representation of identified (super)families of drug efflux pumps in E. coli: ABC, MATE, MFS, PACE, SMR and RND. The arrows represent the pathway of the substrate (drug) or proton movement (in case of ABC-transporters: ATP-hydrolysis) in comparison with each other. The members of ABC MATE, MFS, PACE and SMR (super)families are known to function as single component pumps but some members can form tripartite complexes (e.g., AcrAB-TolC, MacAB-TolC and EmrAB-TolC, of the RND, ABC and MFS superfamily, respectively). Figure was made with help of BioRender (https://app.biorender.com/). 


\subsubsection{The ATP-Binding Cassette Superfamily (ABC)}

The members of the ATP-Binding Cassette (ABC) Superfamily are conserved from bacteria to humans and for the export of compound they use energy from ATP hydrolysis (Figure 5). Transporters are having a nucleotide binding domain (NBD) in the cytoplasm, for ATP binding and hydrolyses, and a transmembrane domain (TMD), for the transport of the substances (drugs, sugars, amino acids, ions, vitamins, proteins, polysaccharides). Members are monomers, homodimers or heterodimers and they can import or export from the cytoplasm. Several structures of ABC transporters have been shown until now, and recently the structure of the tripartite complex that is a member of this family, MacAB-TolC, has been solved (Fitzpatrick et al., 2017).

\subsubsection{The Multidrug and Toxic compound Extrusion Superfamily (MATE) and the Proteobacterial Antimicrobial Compound Efflux family (PACE)}

The Multidrug and Toxic compound Extrusion superfamily (MATE) transporter are found in many bacterial species as well as in animals, plants and humans. Their characteristics are the 12 TM helices (TMH) and the sodium and proton antiport across the inner membrane as a source of energy for transport activity. The members are divided into the NorM, DinF and eukaryotic subfamilies, on the basis of their amino acid sequence similarity (Du et al., 2018). Representative of MATE, NorM of Vibrio parahaemolyticus, with $12 \mathrm{TMH}$ as well, pumps out fluoroquinolones and ethidium in exchange for the influx of sodium ions (Morita et al., 1998). For the member of DinF subfamily, DinF-BH, it has been shown that the substrates are bound in the central cavity. The residue D40 is highly conserved within the DinF subfamily and is involved in the proton transport. D40 is located within a membraneembedded chamber with numerous hydrophobic amino acids and is effectively shielded from the solvent-exposed cleft by TM7 and TM8. Its side-chain, carboxylate, has a calculated pKa of 7.4 and can possibly undergo cycles of protonation and deprotonation as substrates are transported through the transporter in vivo (Lu et al., 2013)(Adam et al., 2007)(Bas et al., 
2008). This was a clear indication that this subfamily needs protons for the substrate transport (Figure 5).

The Proteobacterial Antimicrobial Compound Efflux family is a newly found family of efflux pumps (Figure 5). It was discovered in Acinetobacter and the first identified member was named AceI (the Acinetobacter chlorhexidine efflux protein)(Hassan, Elbourne, et al., 2015). It has been shown that PACE proteins energize the transport of their primary substrates using proton-motive force (Hassan et al., 2019). The members of this family are shown to be responsible for resistance towards synthetic bactericidal agents, like chlorhexidine, acriflavine, proflavine and benzalkonium (Hassan, Liu, et al., 2015)(Du et al., 2018).

\subsubsection{The Small Multidrug Resistance family (SMR)}

The proteins from the Small Multidrug Resistance family (SMR) induce resistance towards quaternary ammonium compounds (benzalkonium) and lipophilic cations (for example, ethidium) in archaea and bacteria (Figure 5). They are proton-drug antiporters. Transporters form this family are found to be homodimers (e. g. EmrE from E. coli) or heterodimers (e. g. YdfEF from E. coli). The representative family member, EmrE, has been shown to be an asymmetric, antiparallel homodimer. The obtained functional data of EmrE (using solution NMR dynamics) combine the asymmetric EmrE structure with the functional symmetry of residues in its active site (Morrison et al., 2012)(Du et al., 2018).

\subsubsection{The Major Facilitator Superfamily (MFS)}

The Major Facilitator Superfamily (MFS) is a large and diverse group of secondary active transporters that are involved in importing and exporting substances out of Gram-negative bacteria, plants and mammals. Members of this superfamily are either single component or tripartite systems. Most members are involved in the transport of sugars; however, several are involved in the transport of drugs, which makes them a part of the antibiotic resistance mechanism (Pasqua et al., 2019). Multidrug efflux is enabled by members of 
drug: $\mathrm{H}^{+}$antiporters 1 (DHA1) and drug: $\mathrm{H}^{+}$antiporters 2 (DHA2) families. These transporters contain 400 to 600 amino acid residues and comprise 12-14 TM segments, organized in two domains and each domain consists of six helices (Radestock \& Forrest, 2011)(Du et al., 2018). Until now, MFS members are known to be proton-drug antiporters. The single component MFS pump, MdfA from E. coli (DHA1), is part of the multidrug resistant phenotype with regard to cationic dyes and chloramphenicol. Deletion of this gene in $E$. coli showed hypersensitivity towards changes in the $\mathrm{pH}$, especially towards alkaline conditions. The activity of MdfA upon changes in $\mathrm{pH}$ is dependent on potassium or sodium ions (Lewinson et al., 2004) (Figure 5).

\subsubsection{The Resistance-Nodulation-(cell) Division (RND) superfamily}

The members of the Resistance-Nodulation-(cell) Division (RND) superfamily are widespread in all domains of life and are responsible for efflux of a variety of substrates (antibiotics, detergents, chemotherapeutics, toxic dyes, etc.). Homologues are found in animals and humans (e. g., Niemann-Pick C1 Like 1 protein) (Altmann, 2004) as well as in Gram-negative bacteria. Important to note is that not every member transports the same substrates. MexB from P. aeruginosa and AcrB from E. coli are not transporting aminoglycosides whereas the homologue AcrD from E. coli does transport this type of substrate (Aires \& Nikaido, 2005). This way, these superfamily members can help bacteria survive many different condition changes in the environment and, together with outer membrane permeability, help development of the resistance towards so many compounds. This makes the RND efflux pumps enormously clinically relevant (Figure 5).

Most studied members of the RND superfamily, AcrB from E. coli and MexB from $P$. aeruginosa, were analysed structurally and functionally. The structure of AcrB was first solved at the resolution $3.5 \AA$ by Murakami et al. (Murakami et al., 2002). This structure showed a symmetrical (LLL) trimer - which is considered the resting state of AcrB (Ruggerone et al., 2013). AcrB can be dived in three domains, the $\mathrm{H}^{+}$-coupling transmembrane domain, the substrate binding and transporting periplasmic porter domain and a more distal periplasmic docking or funnel domain. For both AcrB and MexB asymmetric 
structures were solved, where each of the protomers adopts a different conformation (Murakami et al., 2006)(M. A. Seeger, 2006)(Sennhauser et al., 2006)(Sennhauser et al., 2009). The roles of the different protomer conformations in substrate transport are further discussed below $(1.6 .1,1.6 .3)$.

\subsection{Metal-ion resistance in $E$. coli}

People have been using metals for a very long time, 5000 years, for a variety of purposes. The most widely used metals and metalloids are mercury, arsenic, copper, silver, zinc, which are present also in inorganic water-soluble compounds. In the $19^{\text {th }}$ century, with the development of chemistry, heavy metals started to be used in medical treatments (Pal et al., 2017). Antimicrobial metals have a broad spectrum of antimicrobial activity. Antimicrobial metals have been present in the environment for billions of years, through geological events and usage in medicine, agriculture and manufacturing. This kind of environment was a perfect place for microorganisms to develop resistance towards metal in order to survive. The first reports of acquired antimicrobial metal resistance in bacteria were made in the 1960s with mercury-resistant Staphylococcus aureus isolated from wounds (Moore, 1960).

The effects of silver on bacteria have been investigated for more than 80 years. Extrusion of metal ions is not just some passive diffusion, but it is an energy driven process that involves efflux pumps that are powered either by ATP-hydrolysis or by proton motive force (Nies, 1999). Silver resistance and silver-resistant bacteria were found where silver toxicity can be expected, from burn wards of hospitals where silver salts are used as antiseptics to treat burns, clinically silver-coated catheters, silver-coated wound bandages, polluted soil in mine surroundings, and many more (Silver, 2003). Silver is also used in the laboratory for the detection of proteins on polyacrylamide gels. $\mathrm{Ag}^{+}$binds to denatured proteins forming salts between -COO-groups (Asp and Glu) or by the complex formation of silver ions and nucleophilic groups such as imidazole (His), $-\mathrm{SH}$ (Cys), $-\mathrm{SCH}_{3}$ (Met) and - $\mathrm{NH}$ (Lys) (Rabilloud, 1990). The silver in stained gels is in form of the reduced polymeric Ag (0). 


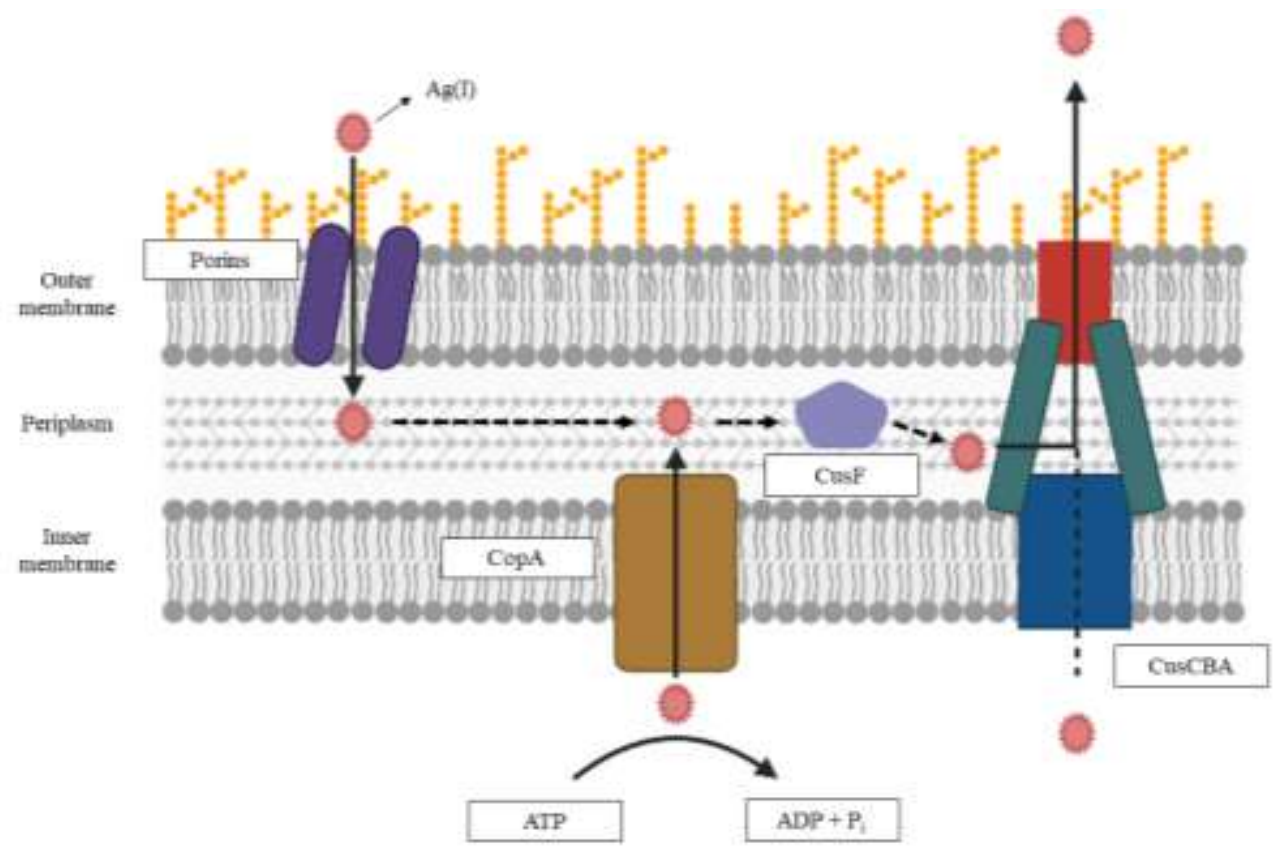

Figure 6. Proposed mechanism of silver efflux in Gram-negative bacteria. Silver ions are taken from cytoplasm and periplasm via chaperons to the transporters that would export ions out of the cell. Figure was made with help of BioRender (https://app.biorender.com/).

Binding to histidine residues, for example, is followed by stabilization of the polymeric $\mathrm{Ag}$ centre, where multiple Ag reduction events happen (Heukeshoven \& Dernick, 1985). One can only imagine how powerful an organism is that confers both antibiotic and metal resistance. That makes it even more important to understand the mechanism that lays under the transport of both metal ions and antibiotics.

One transporter from $E$. coli that is a member of a subfamily of transporters of cations of soft Lewis acids, CopA, is a participant in the copper and silver homeostasis and is involved in efflux of the mentioned ions (Rensing et al., 2000). CopA (834 amino acids, $87.87 \mathrm{kDa}$ ) is incorporated in the inner membrane of $E$. coli, and exports silver/copper ions from the cytoplasm to the periplasm and is regulated by CueR which activates with the change in the copper or silver levels. CopA uses ATP hydrolysis as energy for the transport of metal ions (Figure 6). 


\subsection{Tripartite efflux pumps of $E$. coli}

\subsubsection{The RND efflux pump, AcrAB-TolC}

The role of the RND transporters in the multidrug resistance comes also from the fact that these pumps become associated with the other classes of proteins: outer membrane protein (OMP) and membrane fusion protein (MFP).

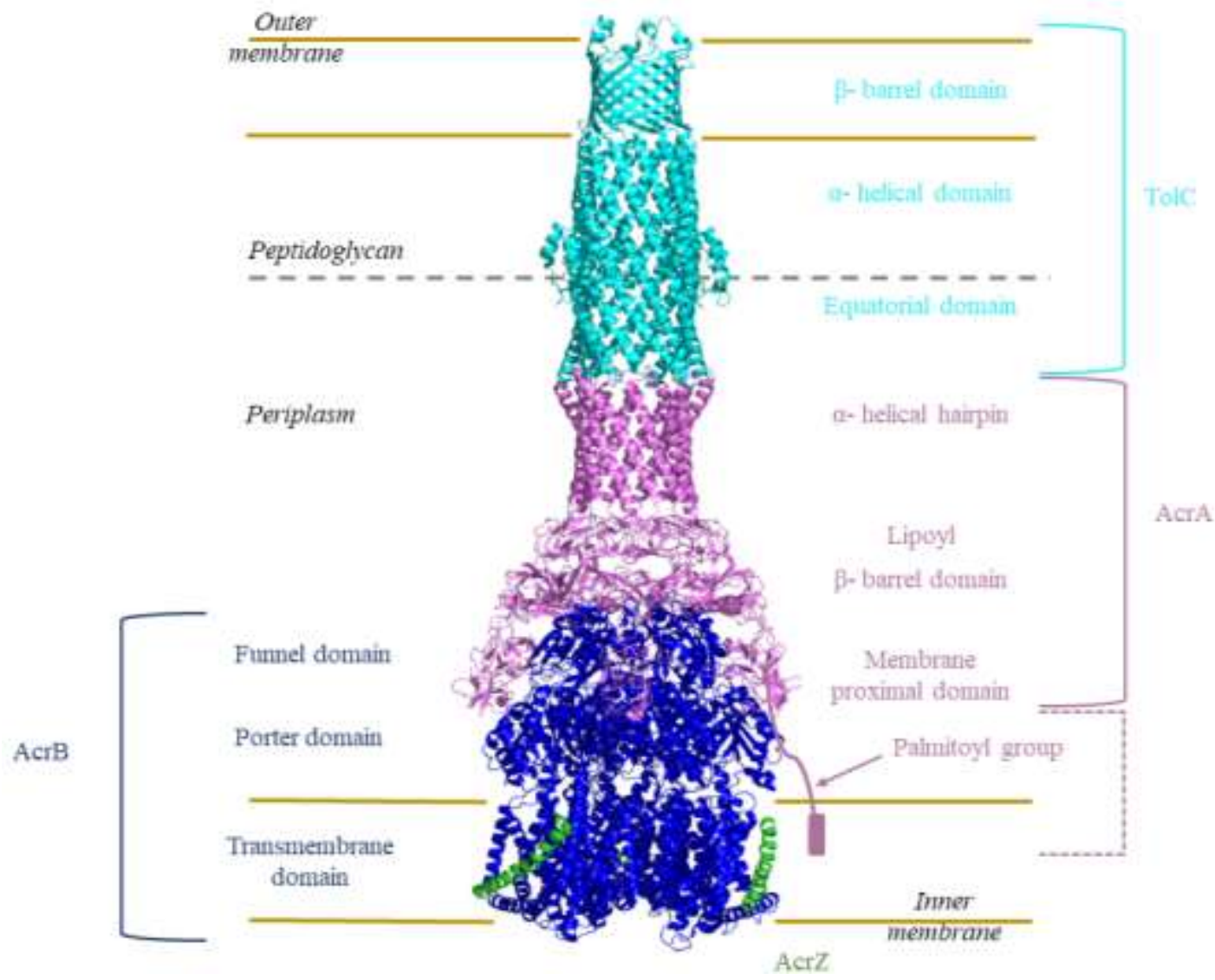

Figure 7. The organization of AcrABZ-TolC complex from E. coli (PDB: 5O66). A schematic diagram of the Gram-negative cell envelope is indicated, containing the inner and the outer membrane as well as the periplasm. In the scheme, a suggested location of the peptidoglycan layer and a proposed position of TolC equatorial domain is shown. Each component of the pump is shown in one colour: AcrA- magenta, AcrB- blue, TolC- cyan, and AcrZ- green. For each component, respective subdomains are shown. AcrA has $\alpha$-helical hairpin, lipoyl, $\beta$ - barrel and membrane proximal domain. AcrA has additionally the anchor to the inner membrane, palmitoyl group, at Cys25. AcrB has periplasmic (funnel and porter domain) and transmembrane domains. TolC has $\beta$ - barrel, $\alpha$ - helical and equatorial domains. Figure was prepared with the help of Pymol (https://pymol.org/). Adapted from (Du \& Luisi, 2015). 
In the case of AcrB, it binds with TolC (OMP) and AcrA (MFP). AcrB was found to interact with the small membrane acriflavine resistance protein $\mathrm{Z}$, AcrZ (49 amino acids, $5.3 \mathrm{kDa}$ ). AcrZ consists of a single tilted helix inside the membrane and thereby fits into a wide groove in the AcrB TMD (Wang et al., 2017).

Acriflavine resistance protein $\mathrm{B}$, AcrB, (1049 amino acids, $113.6 \mathrm{kDa})$ is a RND member conferring resistance to a variety of compounds: colouring agents and dyes, detergents, macrolide antibiotics, $\beta$-lactams, bile salts, solvents, anti-cancer drugs, novobiocin, fusidic acid, rifampicin, chloramphenicol, ciprofloxacin, linezolid, minocycline, tetraphenylphosphonium. Structural work enabled vision of the main characteristics of the pump: asymmetric trimers, where every protomer undergoes a different conformational state. When describing the conformational states, their roles were taken into consideration - the access, the binding and the extrusion of substrates.

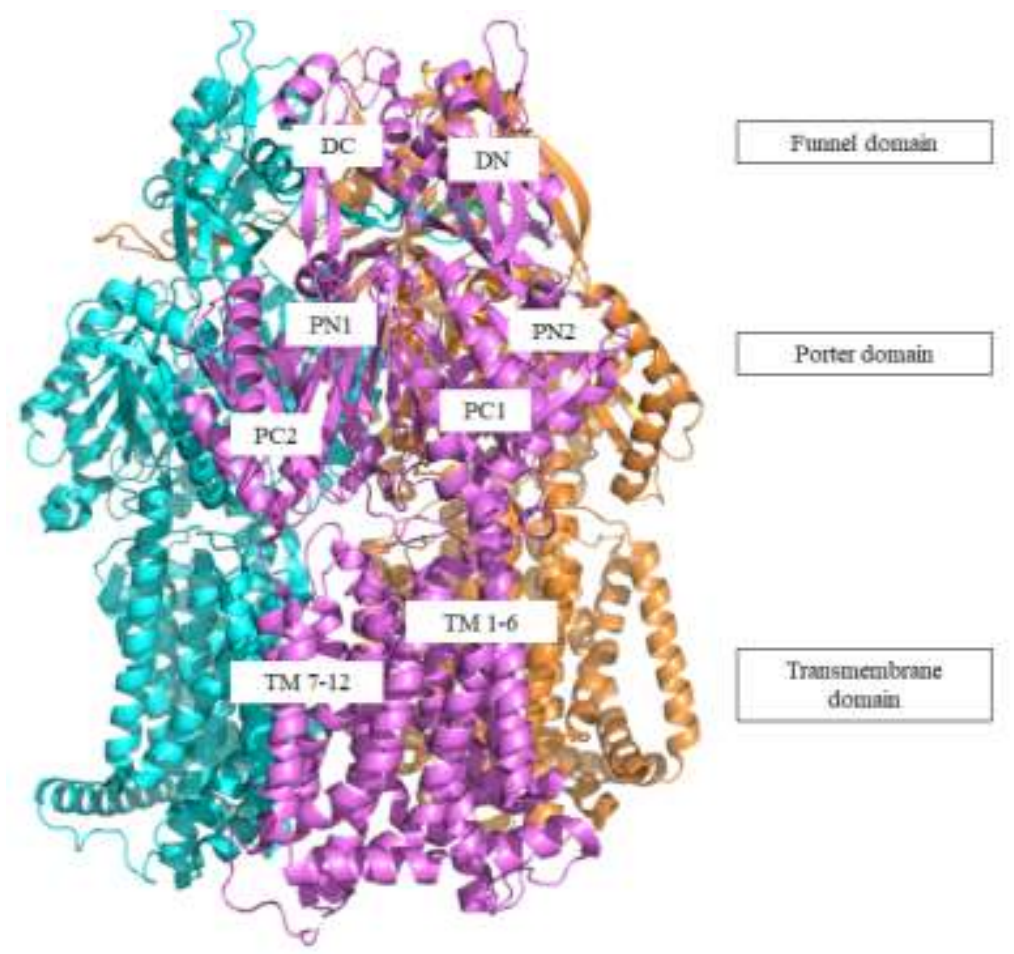

Figure 8. Structural characteristics of efflux pump AcrB (PDB: 4U8V). Side view of the asymmetric AcrB indicating three monomers, each shown in different colours (orange, violet and cyan). AcrB has periplasmic (funnel and porter domain) and a transmembrane domain. The four subdomains PN1, PN2, PC1 and PC2 in the porter domain, as well as two subdomains, DC and DN, in the funnel domain are shown. Positions of the transmembrane helices TM 1-6 and TM 7-12 are indicated. Figure was prepared with the help of Pymol (https://pymol.org/). Adapted from (Ruggerone et al., 2013). 
Respectively, states are called: access, binding and extrusion (Murakami et al., 2006) or alternatively Loose (L), Tight (T) and Open (O) (M. A. Seeger, 2006), in analogy to the states found in the $\mathrm{F}_{1} \mathrm{~F}_{\mathrm{o}}$ ATP synthase (Boyer, 1997). The pump is a substrate-proton antiporter and is composed of three domains: a periplasmic porter domain (PD) (recognition of substrate), a periplasmic funnel domain, and a TM domain (proton translocation) (Figure 8) (Nikaido, 2011)(Pos, 2009)(Kobylka et al., 2020).

The periplasmic part of the transporter is divided in the porter domain and funnel domain (also called docking domain). The porter domain is divided in four subdomains: PN1, PN2, PC1 and PC2, where PN1/PC2 and PN2/PC1 are structurally coupled. The PN2, PC1 and PC2 subdomains are laterally exposed to the periplasm, with a large solvent accessible cleft between PC1 and PC2 and the PN1 subdomain positioned at the interior of the AcrB trimer includes a central helix, that together with the central helices of PN1 subdomains of the other protomers forms a central pore. The funnel domain is divided in the subdomains DN and DC. The DN domain has a loop that extends towards the DC domain and is crucial for the AcrB trimerization (Cha et al., 2014). The funnel domain comprises a cavity, which is open to the periplasm and is suggested that is a pathway for the substrates to be transported into TolC/AcrA channel (Pos, 2009)(Du et al., 2015). The transmembrane domain contains 12 transmembrane helices (TMH) as well as two large periplasmic loops between TM1 and TM2, and between TM7 and TM8. The TM domains of every AcrB protomer make a central cavity (30-35 ̊), which is packed with lipids (Thomas Eicher et al., 2014)(Qiu et al., 2018).

AcrB substrates are being captured from the periplasm and from the outer leaflet of the inner membrane bilayer (Pos, 2009). Substrate transport by AcrB is described to happen via a functional rotation in which each protomer goes through a conformation change (L, T and $\mathrm{O}$ ). AcrB, a secondary active transporter, couples the efflux of substrate with the influx of protons. Two hydrophobic drug binding sites were described in AcrB: the access pocket (AP) and deep binding pocket (DBP), that have a flexible switch-loop between them (T. Eicher et al., 2012)(Thomas Eicher et al., 2014)(Cha et al., 2014).

During the L to $\mathrm{T}$ transition, the rearrangement of PN2/PC1 repeat happens (the repeat in the porter domain), which makes the downward movement of TM2 leading to the gap of the TMD towards the periplasmic site allowing proton entry via a water molecule network. Residues D407, D408 (TM4) and K940 (TM10) are forming a charged interface between two transmembrane repeats (TMR1: TM1+TM3-6; TMR2: TM7+TM9-12). The entry of protons 
leads to the protonation of D407 and D408. Their protonation happens during transition from $\mathrm{T}$ to $\mathrm{O}$ and substitution of these residues with not titratable residues results in the disabling AcrB to transport its substrates (Figure 9) (Thomas Eicher et al., 2014).

The coupling elements between the PD and the TMD are the helices TM2 and TM8; TM2 is connected to the PN2/PC1 repeat in the PD and TM8 is connected to the PN1/PC2 unit. The ionic network between D407, D408 and K940 breaks apart and K940 then orients to TM11 to compensate its charge with N941, T978, as well as with a water molecule. This reorientation leads to the upward movement of TM2 (since it involves the entire transmembrane repeat 2 including TM10). This upward movement closes the deep binding pocket. The TM rearrangement also affects the orientation of TM8 - the connected PN1/PC2 subdomains move to the PN2/PC1, and close the access binding cleft towards the periplasm. In the $\mathrm{O}$ state, the TM domain is open towards the cytoplasm. When the reorientation of R971 happens, that would change the pKa values of D407 and D408. This leads to the proton releasing to the cytoplasm. When the residues D407, D408 and K940 re-engage, TM2 and TM8 take their initial positions entering again into the $\mathrm{L}$ state. When the proton is released, the subdomain repeats $\mathrm{PN} 1 / \mathrm{PC} 2$ and $\mathrm{PN} 2 / \mathrm{PC} 1$ reorient and the access pocket reopens towards the periplasm (Thomas Eicher et al., 2014). 

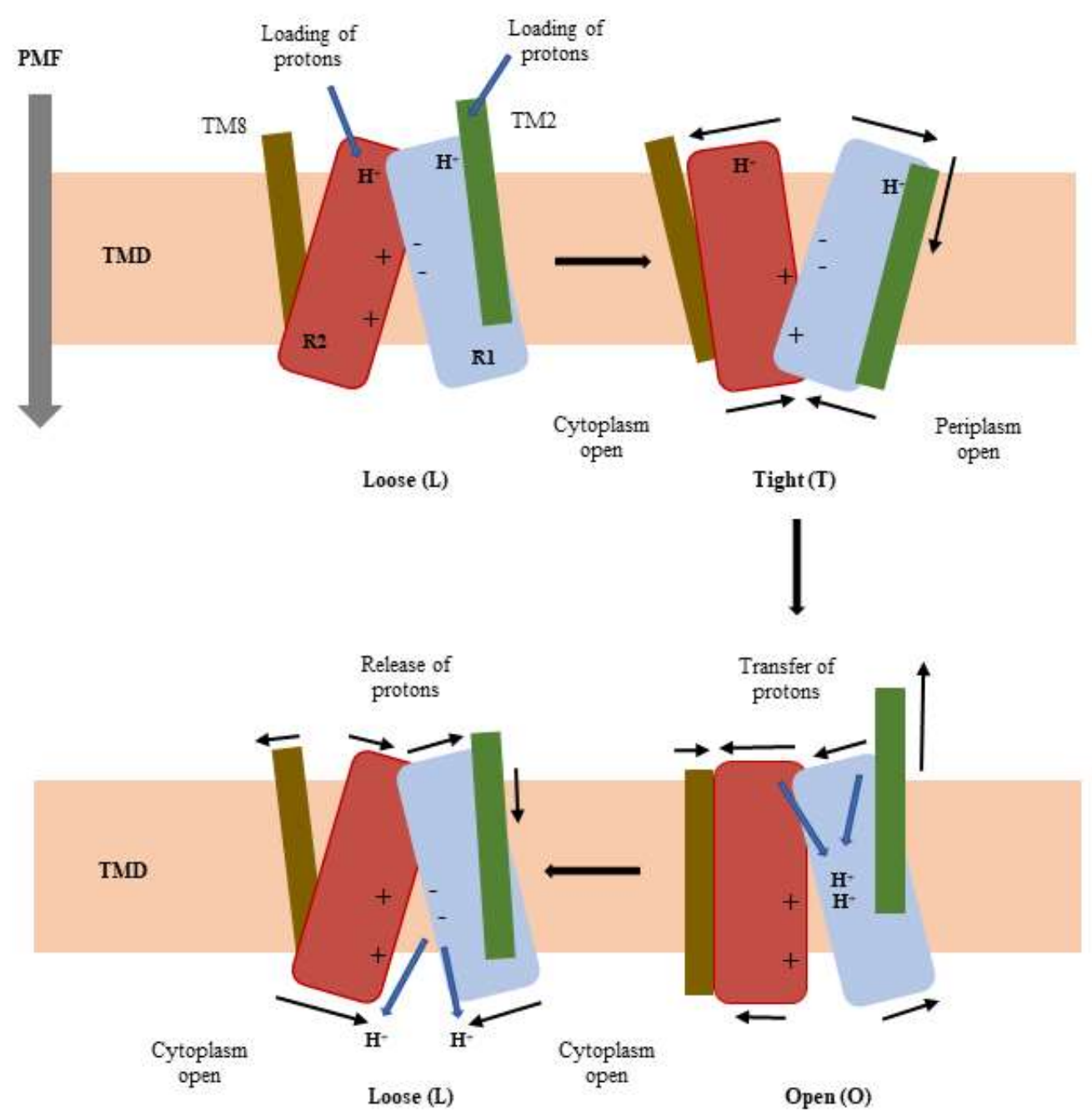

Figure 9. Suggested mechanism of proton transport through AcrB. The schematic representation of the proton pumping activity of AcrB is shown: R1, R2- structural repeats in the transmembrane (TM), light blue and red, respectively; TM2, TM8- TM helices 2 (green) and 8 (brown), respectively; TMD- transmembrane domain, seen from a membrane plane; PMFproton motive force. The conformational states Loose $(\mathrm{L})$, Tight $(\mathrm{T})$ and Open $(\mathrm{O})$ are indicated. Residues in the proton relay network- K940, R971, D407 and D408, are represented with "+” (positive) and "- "(negative) charges. Adapted from (Thomas Eicher et al., 2014).

Acriflavine resistance protein A, AcrA, (397 amino acids, $42.2 \mathrm{kDa}$ ) is a membrane fusion protein. The mature AcrA that lacks signal peptide is anchored then to the inner membrane via palmitoylation at Cys25 (H. I. Zgurskaya \& Nikaido, 1999). According to the tomography results and in vivo interactions, AcrA and AcrB associate to form a bipartite complex and the contact between the $\alpha$-hairpin domain of AcrA and the peptidoglycan layer is the one that gives stability to the AcrAB position and formation (Shi et al., 2019). AcrA forms a hexamer. Each protomer has subdomains (Figure 7). Only the $\beta$-barrel and the membrane proximal 
domain (MBP) are in direct contact with AcrB. AcrA connects AcrB and TolC and is necessary for the activity of the whole tripartite pump assembly (Du et al., 2014).

The outer membrane protein, TolC, (493 amino acids, $53.7 \mathrm{kDa}$ ) is involved in the extrusion of the small molecules and toxins across the outer membrane of Gram-negative bacteria (Helen I. Zgurskaya et al., 2011). It is a homotrimer and forms a 12-stranded $\beta$-barrel pore domain. Its opening merges into a $100 \AA$ long $\alpha$-barrel periplasmatic domain that consists of a total 12 alternating continuous and pseudo-continuous $\alpha$-helices. TolC was crystallized at 2.1 A resolution in a closed state, which is the state of the isolated wild type protein, where the helices at the lower end of the $\alpha$-barrel are twisted into each other - a closed iris diaphragm (Koronakis et al., 2000). It has been shown that AcrAB recruits TolC in the presence of the substrate and that both AcrA and TolC interact with the peptidoglycan in the complex (Shi et al., 2019).

In the absence of substrate, TolC in the apo-state is closed while the AcrA protomers are unsteady packed without sealing the cavity towards the periplasm. In the presence of substrate, AcrB adopts the asymmetric conformation resulting in the repacking of the AcrA $\beta$-barrel and MPD and the reorientation of the $\alpha$-helical coiled-coil hairpins. The hairpins of AcrA thereby form a tip-to-tip interaction with the tips of the $\alpha$-barrel at the lower end of TolC. This event causes a AcrA-induced opening of TolC (the iris-like opening) and at the same time seal the AcrA tunnel cavity towards the periplasm. The active AcrAB-TolC complex will compress $10 \AA$ in length compared to its pre-active tripartite state, where TolC is still closed (Shi et al., 2019). In conclusion, the entire tri-component efflux pump seems to be a highly allosteric machinery where the conformational changes in the inner membrane component are being linked to the outer membrane protein via the membrane fusion protein (Kobylka et al., 2020)(Wang et al., 2017). This conclusion is in line with the work done on a homologues pump MexAB-OprM from P. aeruginosa, where it is suggested that the mechanism of an opening of OprM might be similar to the one of TolC and depends on the MexA-OprM connection. The MexA and OprM are also suggested to have a more important role then only being a channel, by allosterically enhancing MexB-mediated drug transport in the transport-competent tripartite form (Tsutsumi et al., 2019)(Glavier et al., 2020). 


\subsubsection{The MFS efflux pump, EmrAB-TolC}

Most of the members of MFS superfamily do not form tripartite systems, in contrast to the RND superfamily members. However, it has been shown for one MFS member that it does form a tripartite efflux pump, for more efficient extrusion of drugs. This tripartite pump is consisting of the inner and outer membrane component (IMP and OMP, respectively) and MFP as well. That pump, EmrAB-TolC from E. coli, is briefly examined in this study and consists as an assembly of EmrA (MFP; 42.7 kDa, 390 amino acids) and EmrB (IMP; $55.6 \mathrm{kDa}, 512$ amino acids) and TolC (OMP; $53.7 \mathrm{kDa}, 493$ amino acids).

MFS-transporter EmrB component is predicted to contain 14 TMH (O. Lomovskaya \& Lewis, 1992). The first electron microscopy insights in the EmrAB-TolC from E. coli were obtained after reconstitution of purified complex in Amphipol A8-35 (Apol). This study showed that the entire EmrAB-TolC system is about $33 \mathrm{~nm}$ in length, similar to AcrAB-TolC and MacAB-TolC. EmrB is predicted not to contain any considerable periplasmic domains. The densities obtained between the periplasmic end of TolC and the second Apol belt are described to belong to EmrA (Yousefian et al., 2021).

The crystal structure of an MFS pump adaptor, EmrA from Aquifex aeolicus, shows arrangement consistent of: $\alpha$-helical coiled-coil, lipoyl, and $\beta$-barrel domains. However, it is lacking the fourth domain i.e., the MPD which was shown in other pumps (e.g., AcrA from E. coli) to interact with the IM transporter. The structure was obtained at $2.85 \AA$ resolution. The $\alpha$-hairpin is exceptionally long (127 ̊) (Hinchliffe et al., 2014). The length of the periplasmic region of EmrA from E. coli which is about $15 \mathrm{~nm}$ is shorter than the length described for the EmrA homolog from Aquifex aeolicus (18.5 nm) (Figure 10). Given the length of the EmrA from E. coli, the interaction with the periplasmic $\alpha$-helical barrel of TolC might most likely correspond to a "tip-to tip" manner of interaction. The oligomeric state of EmrB is still not known, even though it is assumed that it functions as a dimer (Yousefian et $a l ., 2021)$. TolC is present in the system as a trimer and EmrA densities suggest a hexameric arrangement (Yousefian et al., 2021). 


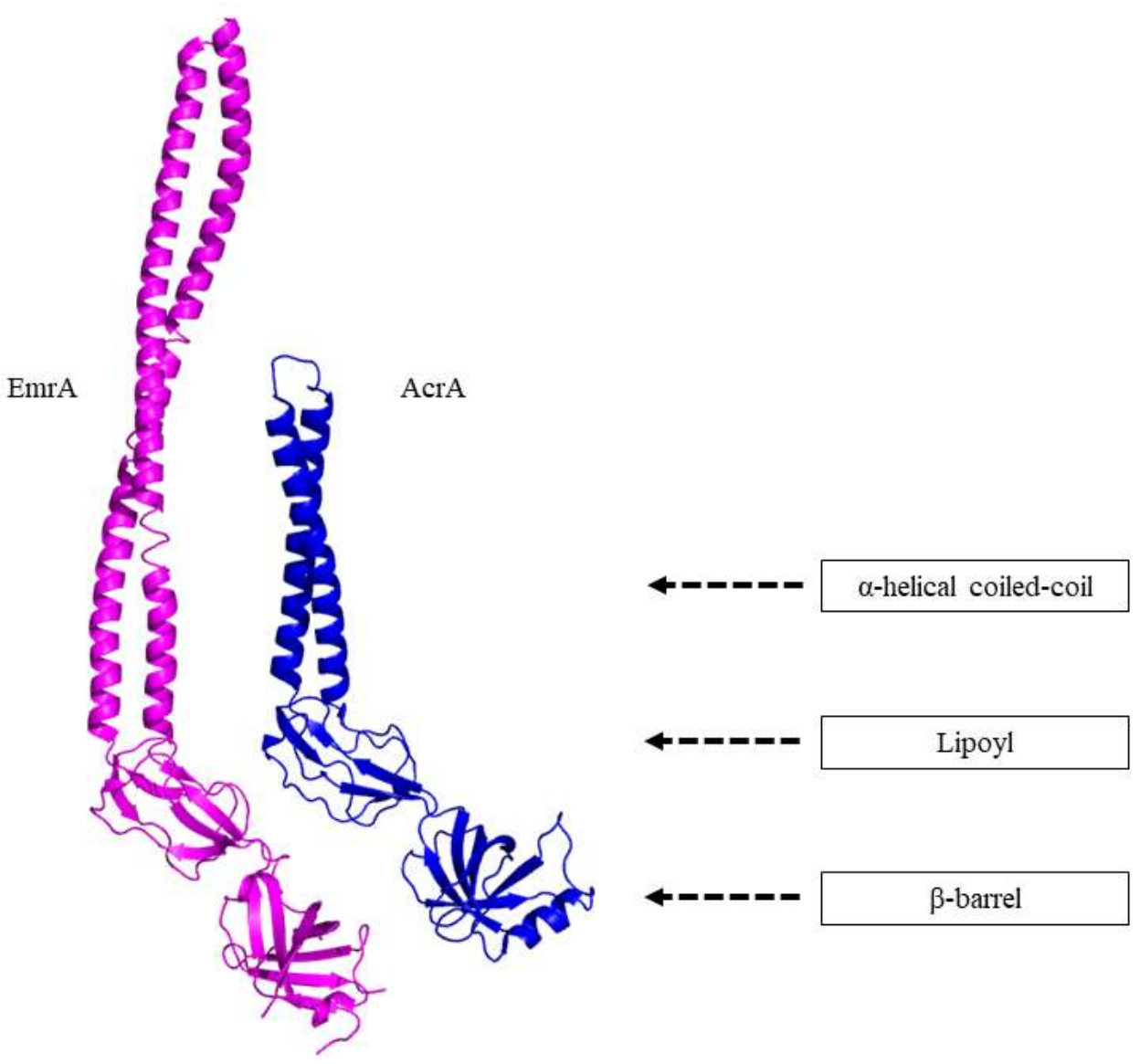

Figure 10. Structure of EmrA from Aquifex aeolicus (PDB: 4TKO; in magenta). For comparison, the structure of AcrA from E. coli is shown (PDB: 2F1M; in blue). The $\beta$-barrel, lipoyl and $\alpha$-helical coiled-coil domains are indicated. The $\alpha$-helical coiled-coil of EmrA from E. coli is shorter by 45 amino acids than EmrA from A. aeolicus. EmrB is predicted not to contain any substantial periplasmic domain(s) unlike AcrB, which explains the length of the hairpin domain that is longer than the one from AcrA. Figure was prepared with the help of Pymol (https://pymol.org/).

Functionally, EmrAB was first described 29 years ago, when it was shown that the overexpression of the $\operatorname{emr} A B$ operon, due to either induction or by a repressor mutation, results in intrinsic resistance of $E$. coli to carbonyl - cyanide m-chlorophenylhydrazone (CCCP) and nalidixic acid (O. Lomovskaya \& Lewis, 1992)(Furukawa et al., 1993). In vivo studies done with cells overexpressing the pump, confirmed that the EmrAB-TolC system is responsible for resistance against CCCP (Yousefian et al., 2021). 


\subsubsection{The RND metal-ion efflux pump, CusCBA}

Even though most RND proteins are hydrophobic and amphiphilic compound efflux transporters (HAE-RND), the heavy metal-ion efflux (HME-RND) subfamily exports heavy metal cations. A member of this family is CusA. CusA (1047 amino acids, $114.7 \mathrm{kDa}$ ) forms a tripartite complex with a member of the MFP family, CusB (407 amino acids, $44.3 \mathrm{kDa}$ ) and an outer membrane protein, CusC (457 amino acids, $50.3 \mathrm{kDa}$ ). A constructed CusCBA model (with CusBA and CusC) gives the final structural model of CusC3-CusB6-CusA3 (the same as it was found for AcrAB-TolC and MexAB-OprM). The tripartite efflux pump CusCBA is a $750-\mathrm{kDa}$ complex that spans both the inner and outer membrane of E. coli (Figure 11, Figure 6). This complex has high specificity for silver and copper ions $(\operatorname{Ag}(\mathrm{I})$ and $\mathrm{Cu}(\mathrm{I})$ ) (Figure 12). It was postulated that metal ion transport is possible from both cytoplasm and periplasm (Long et al., 2010). While for the cytoplasmic transport metal ions are captured by CusA, for the periplasmic transport metal ions are bound to $\mathrm{CusB}$, which triggers conformational changes in both CusB and CusA thus binding and transporting the ions further. The smaller periplasmic protein, CusF, is a chaperone that shuttles the ions present in the periplasm towards CusB (Figure 6).

Earlier on, the structures of CusA in the presence and absence of $\mathrm{Cu}(\mathrm{I})$ or $\mathrm{Ag}(\mathrm{I})$ have been obtained by X-ray crystallography (Long et al., 2010). CusA forms a trimer, and each subunit of CusA consists of 12 TMH (TM1-TM12) and a large periplasmic domain formed by two periplasmic loops between TM1 and TM2, and TM7 and TM8, respectively. The four helices, TM4, TM5, TM10 and TM11 extend into the cytoplasm where they form the cytoplasmic domain of CusA. On the other hand, TM2 and TM8, extend into the periplasm and are part of the periplasmic domain. 


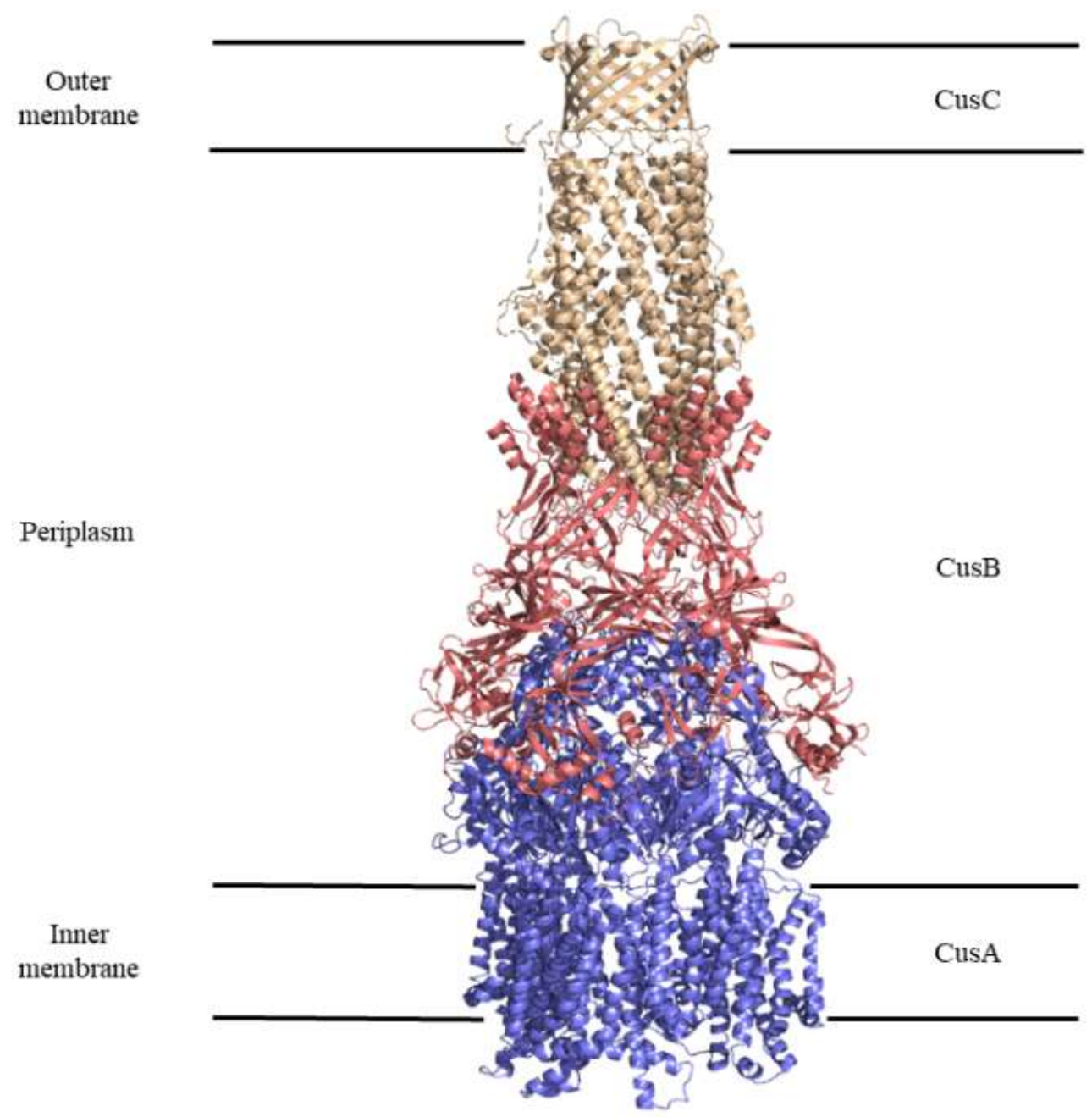

Figure 11. Proposed model of CusCBA efflux pump (PDB: 3NE5 for CusBA; PDB: 3PIK for CusC). The CusCBA complex spans the periplasmic space of Gram-negative bacteria when the cell is under heavy metal ion stress, when it assembles and expels metal ions. Metal ions are delivered to the CusB (shown in deep salmon red) metal-binding sites which in return activates the CusA pump. CusA (shown in slate blue) would take up and transport metal ions towards CusC (shown in wheat yellow), which will extrude them out of the cell. Figure was prepared with the help of Pymol (https://pymol.org/). Adapted from (Chacon et al., 2014).

The periplasmic domain of CusA is divided into six sub-domains, PN1, PN2, PC1, PC2, DN and DC (Long et al., 2010). Monomer 1 of CusB is mainly in contact with the upper regions of PN2 and PC1 and the DN subdomain of CusA while monomer 2 of CusB bridges the upper regions of PC1 and PC2, as well as the subdomain DC of the pump (Figure 12). The trimeric CusA pump directly interacts with six CusB adaptor protomers. The six CusB protomers form a channel at the top of the CusA trimer (Figure 12) (Su et al., 2011). 

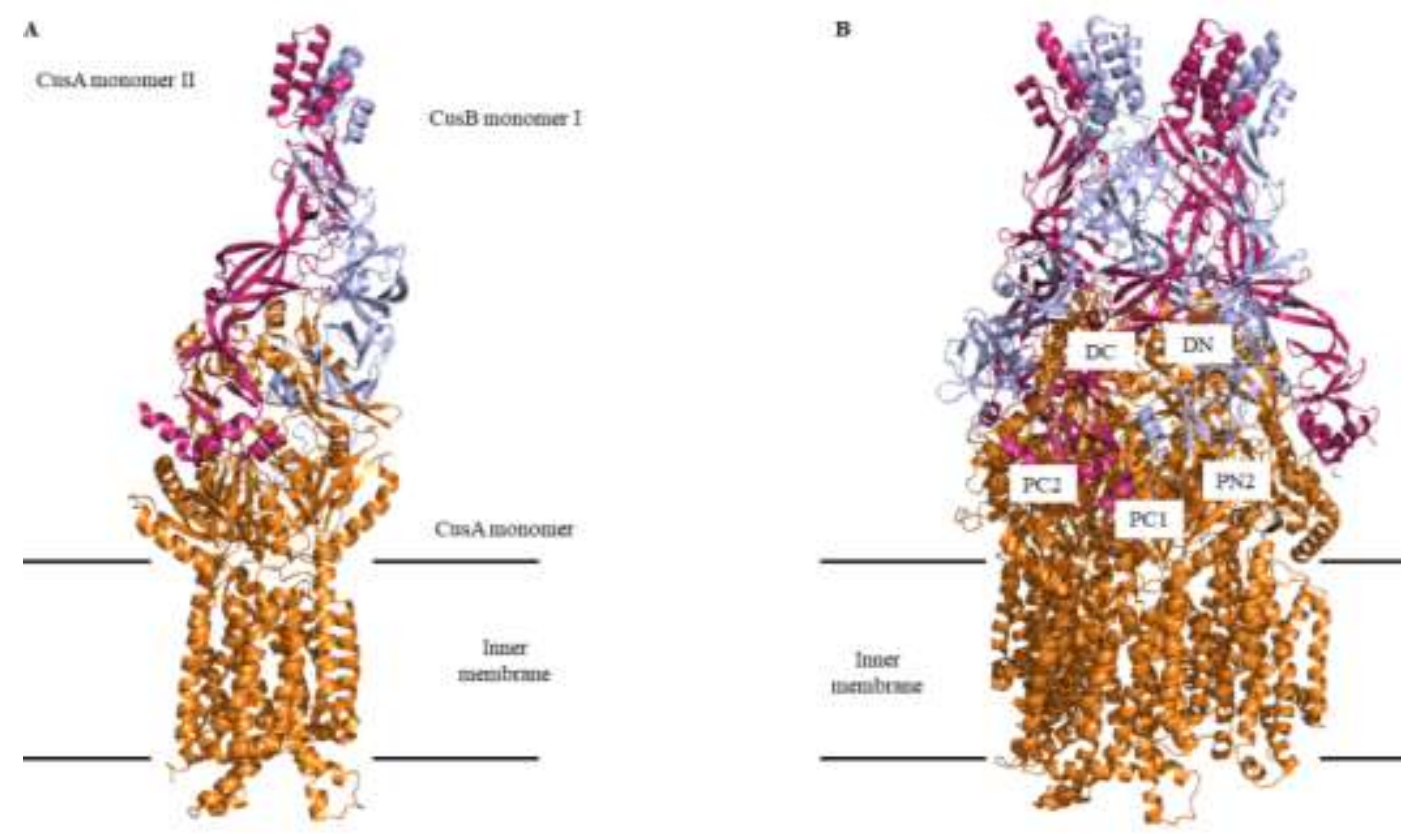

Figure 12. Structure of E. coli CusBA (PDB: 3NE5). A) Two monomers of CusB (magenta and blue) and one monomer of CusA (orange) are shown. B) Side view of the CusBA efflux complex. DC, DN, PC1, PC2 and PN2 are subdomains of CusA. Each subunit of CusA is shown in orange. Molecules of CusB are shown in magenta and blue. Figures were prepared with the help of Pymol (https://pymol.org/). Adapted from (Su et al., 2011).

Crystallographic data without the substrate being bound to CusA, showed the "resting" state conformation, where the periplasmic cleft is closed. Upon the addition of metal ions, both the CusA-Cu(I) and CusA-Ag(I) structures are shown to be in the open cleft conformation, where the PC2 subdomain is found to swing away from the PC1 subdomain by $30^{\circ}$ when compared with the CusA structure in the resting state. This conformational shift allows the periplasmic cleft to open. A single $\mathrm{Cu}(\mathrm{I})$ or $\mathrm{Ag}(\mathrm{I})$ is found to bind in the middle of the transient methionine triad (M573-M623-M672) that is located in the cleft. The CusA-Cu(I) and CusA$\operatorname{Ag}(\mathrm{I})$ structures are nearly identical to each other and represent the "binding" state of the CusA pump (Long et al., 2010).

Recently, cryo-EM structures of CusA in the presence of $\mathrm{Cu}(\mathrm{I})$ have been determinated. In a single cryo-EM sample, four different structures of CusA were present. Three CusA molecules can assemble as symmetric trimers (EEE and BBB trimeric structures, where E stands for "extrusion" and B for "binding"). Asymmetric trimers were also detected as suggested by the EEB (the assembly of two "extrusion" protomers and one "binding" protomer) and EBB (the assembly of one "extrusion" and two "binding" protomers) structures (Moseng et al., 2021). 
In the transmembrane domain of CusA, the conserved residues D405, E939, and K984 are essential for the efflux of metal ions. Seemingly, point mutations in these amino acids make CusA not able to pump metal ions and thus indicate the importance of the proton relay network for the transition between different conformational states (Long et al., 2010).

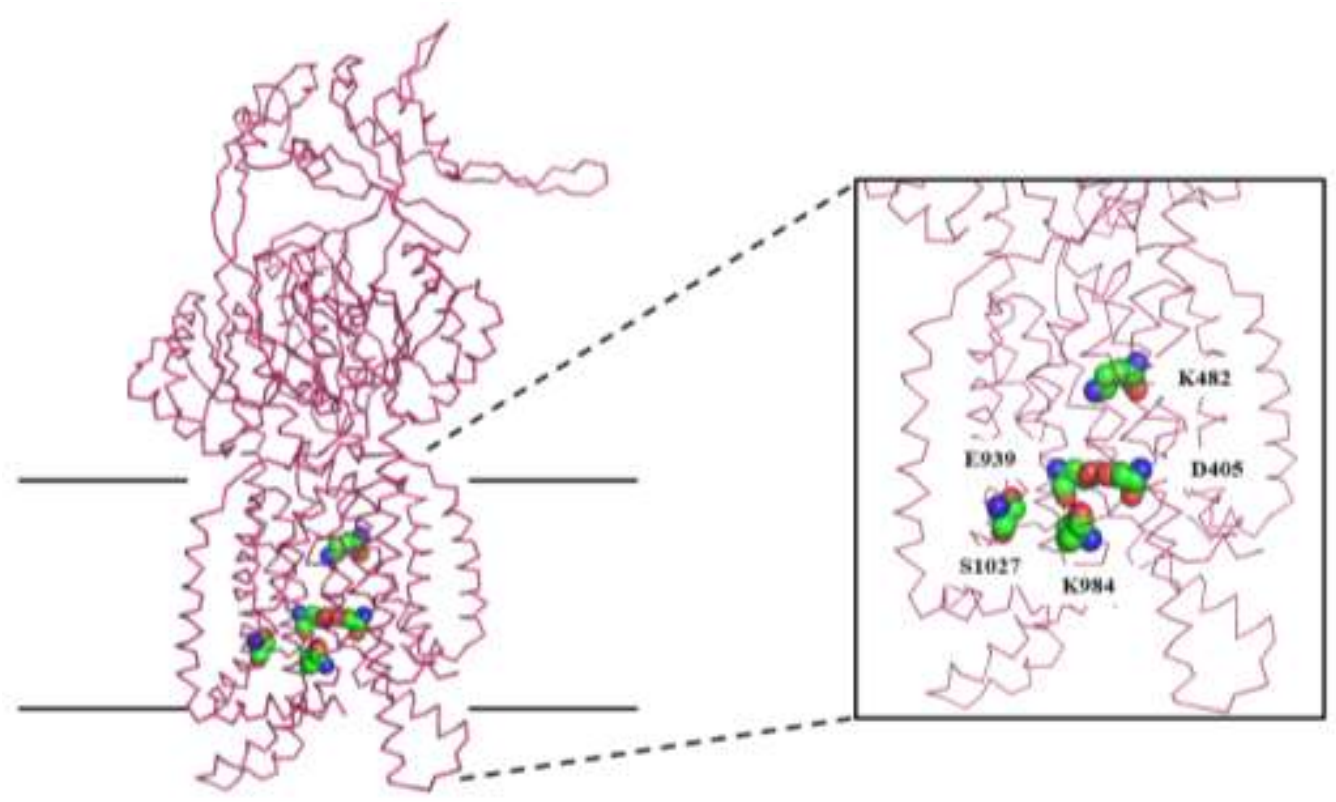

Figure 13. Proton relay network in CusA. An analysis of CusA (pink) showed the residues that are involved in the proton network. The residues D405, E939, K482, K984 and S1027 are shown as spheres. K984 is suggested to play a role in the transfer of protons from D405 to S1027. S1027 transports protons then towards the cytoplasm. Atoms are shown as: O- red, C- green and N- blue. Figure was prepared with the help of Pymol (https://pymol.org/).

The side chain of K984 sweeps away from D405 and shifts toward the cytoplasm and at the same time this alternating side chain orientation is able to create a new hydrogen bond with S1027. The proton can be then effectively passed on from D405 to K984, which then sweeps and gives the proton to S1027. This movement is coupled with the opening of the periplasmic cleft in order to bind $\mathrm{Cu}(\mathrm{I})$ (Moseng et al., 2021) (Figure 13). 


\subsection{Reconstitution, a tool for studying membrane proteins}

\subsubsection{Biological membranes}

Cells and organelles are enclosed with a system of lipids and proteins: biological membranes. The membrane has the function of a semi-permeable barrier that separates the inside and outside of an organism. However, the membrane is not just a separator of different partitions, it is also the complex arrangement that provides energy to the living entity by allowing gradients of ions to be created.

Early experiments by E. Gorter and F. Grendel in 1925 were the first to demonstrate that biological membranes are made as bilayers. By extracting the lipids from red blood cells and measuring them using a Langmuir film balance, they found that they occupied a space that was within the error twice the surface area of the monofilm. The conclusion was made that the membrane of the cell is made of two opposing thin molecular layers (two lipid layers form a bilayer with the polar head groups pointing toward the aqueous environment) (Watson, 2015)(Heimburg, 2009). From the first proposed fluid mosaic model by Singer and Nicolson in 1972, where it was said that the membrane is dynamic and fluid, with lipids and proteins that can laterally diffuse within the membrane, the model had some refinement since it has to be taken into account that distribution of lipids and proteins is not homogeneous. Certainly, this inhomogeneity can produce clusters within the membrane. The Mattress Model by Mouritsen and Bloom in 1984, takes into account that the hydrophobic core of proteins might not match thickness of the bilayer which leads to interfacial tensions and capillary forces between protein and lipids (Heimburg, 2009). The knowledge about the biological membrane is an ongoing study subject. Cell membranes are fully integrated structures that are responding to environmental and internal changes, which makes them dynamic and complex structures.

Main components of the membranes are lipids, proteins and sugars. Lipids are amphipathic molecules; they consist of a hydrophilic polar head and of (a) hydrophobic aliphatic tail(s). Phospholipids, sphingolipids, sterols and glycolipids are the major groups of lipids found in biological membranes. Membranes are composed of amphiphilic lipids which in most cases and conditions studied are glycerophospholipids, composed of fatty acyl chains, a glycerol 
phosphate, and a variable head group. E. coli has been the standard model for studying bacterial membrane lipids. The membranes of $E$. coli comprise three major membrane phospholipids. About $75 \%$ of membrane lipids is zwitterionic phosphatidylethanolamine (PE); in addition, the anionic lipid phosphatidylglycerol (PG) is present with about $20 \%$, as well as cardiolipin (CL) (1-5 \%) (Raetz, 1986).

Lipids play an important part in the membrane. By surrounding membrane proteins, they are considered to influence the activity of the mentioned. The co-dependence between lipids and proteins has been shown in the structural determinations of some membrane proteins, that could not be done unless lipids are present together with the proteins. Lipids in the membrane create the environment for the activity of membrane proteins.

This importance of the lipids must be taken into the consideration when proteins are studied in vitro. Purified proteins that are taken out from the native environment have to be incorporated into the new environment that will mimic the native one. This artificial membrane has to provide enough stability and organization for the proteins to show activity as in the original biological membrane.

Membrane proteins are proteins that are part of the membrane or interact with the membrane, and the understanding of the structure and function of membrane proteins is the key to the process of defeating the diseases that attack our living systems. Membrane lipids form a permeability barrier but specific membrane proteins are mediating almost all other membrane functions. Membrane proteins transport chemicals and information across the membrane. They are involved in gatekeeping (receptors), import of nutrients and ions as well as in the export of xenobiotic compounds (transporters). Another role of membrane protein is a maintenance of the permeability across membrane bilayers (channels). Also, membraneembedded enzymes catalyse chemical reactions.

\subsubsection{Liposomes}

Liposomes are small artificial, spherical vesicles that can be created from synthetized and natural phospholipids. The name - liposome, comes from the two words originated from the Greek language, "lipos" - fat and "soma" meaning body (Daraee et al., 2016). They have one 
or more lipid bilayers surrounding aqueous unites, where the polar head groups are facing towards the aqueous phases present in the interior and exterior of the vesicle (Figure 14).

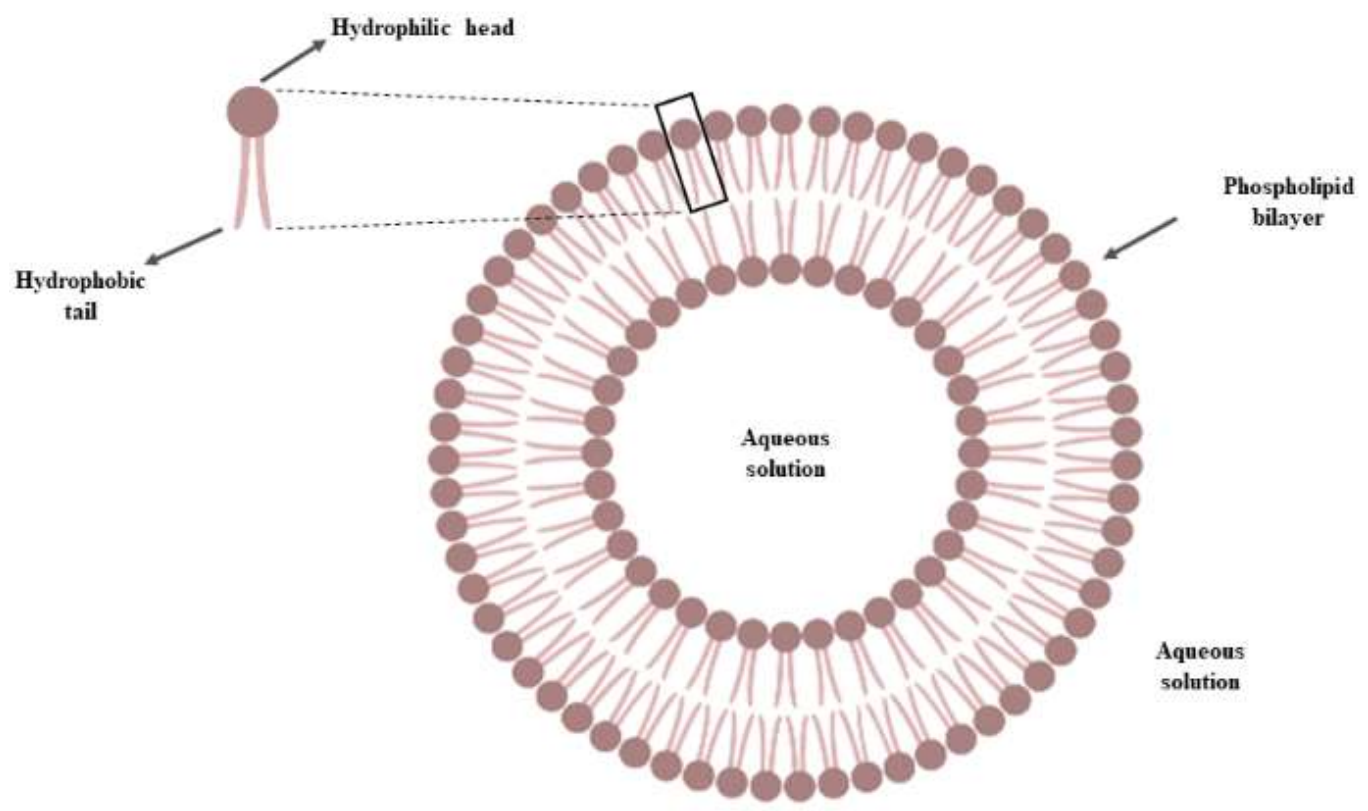

Figure 14. Schematic representation of the bilayer membrane of liposome. A lipid molecule consists of a hydrophilic head and a hydrophobic tail. The tail is oriented towards the inside of the bilayer and the head towards the aqueous surroundings. Figure was made with help of BioRender (https://app.biorender.com/).

Liposomes can be classified according to the number of lipid bilayers and their size. According to the number of the lipid bilayers, liposomes are divided into unilamellar liposomes with one bilayer and liposomes with more than one bilayer, that are called multilamellar vesicles (ULVs or MLVs, respectively (Figure 15)). The latter are between 200 and $1000 \mathrm{~nm}$ in diameter. ULVs are divided into: Small Unilamellar Vesicles (SUVs), with diameters smaller than $200 \mathrm{~nm}$; Large Unilamellar Vesicles (LUVs), with a diameter in between $200 \mathrm{~nm}$ and $1000 \mathrm{~nm}$ and Giant Unilamellar Vesicles (GUVs), with a diameter larger than $1000 \mathrm{~nm}$.

Liposomes were first discovered by British haematologist Alec Douglas Bangham in 1961 at the Babraham Institute, in Cambridge, while him and R. W. Horne were testing the new electron microscope by adding negative stain to dry phospholipids. First reports and protocols that describe the formation of liposomes were published in the following years (Bangham et al., 1965)(Bangham et al., 1974). 


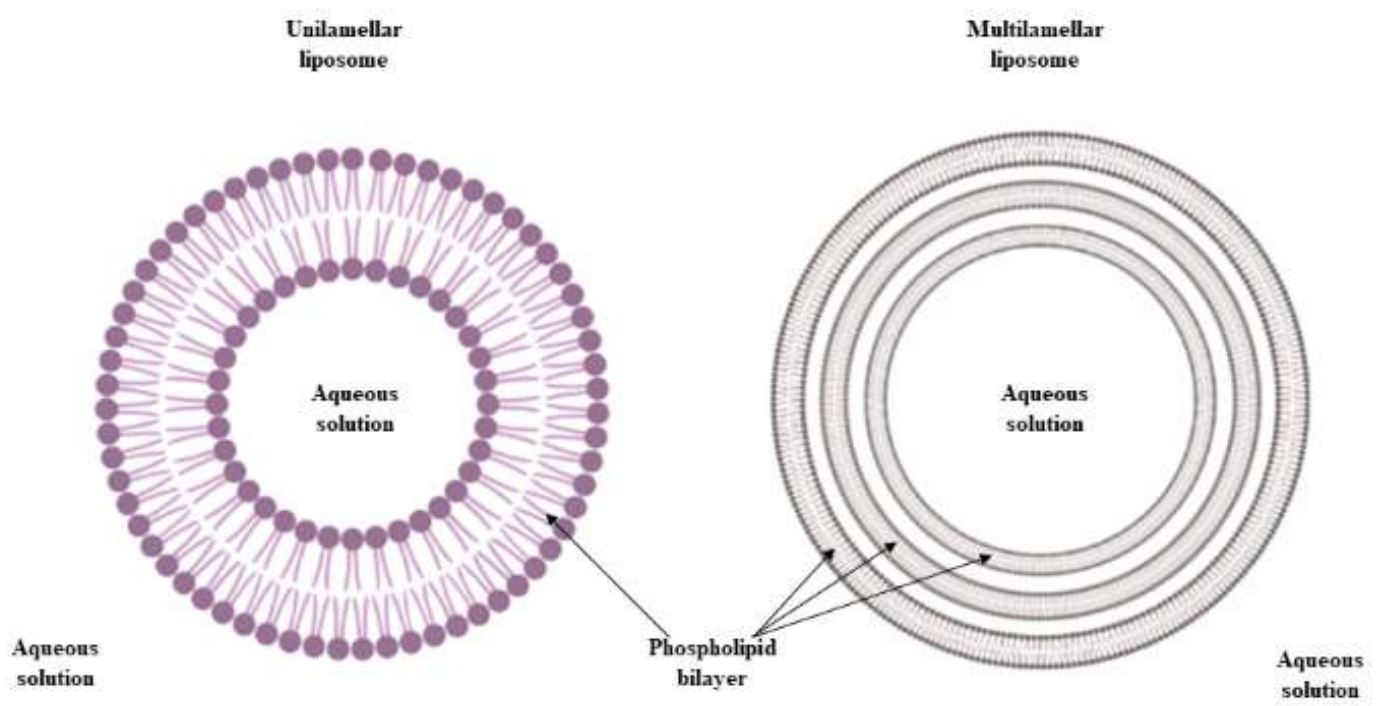

Figure 15. Schematic representation of unilamellar and multilamellar liposomes. Figure was made with help of BioRender (https://app.biorender.com/).

Since then, the usage of liposomes increased, and because of their biocompatibility, biodegradability, low toxicity and capacity to encapsulate hydrophilic and lipophilic drugs, and their ability to simplify site-specific drug delivery to tumor tissues, they are used as an investigation system and a drug delivery system. Studies that want to use low drug toxicity and target specific cells, use liposomes (Hofheinz et al., 2005)(Schiffelers, 2001).

\subsubsection{Preparation of liposomes}

Lipids are usually first dissolved in chloroform that has to be removed by drying. Chloroform is used in order to get the desired homogeneity of the lipid solution. The drying of chloroform is done by applying a vacuum, freezing, or via nitrogen flow. Using any of the methods for the solvent removal has to also unsure that the oxidation of the lipids would be avoided. When the lipid dry film is formed then it has to be hydrated back to solution with an aqueous buffer and vigorous mixing. After this step, the solution contains multilamellar large liposomes (MLVs). For the formation of the other types of liposomes, energy has to be provided and added to the system, e.g., sonication, which then leads to SUVs (de Freitas et $a l ., 2019$ ) and extrusion, leading to formation of LUVs (Hope et al., 1985) (Figure 16). Other methods for liposome formation can include: freeze-thawing, lipid film hydration by hand 
shaking, non-hand shaking or freeze drying, micro-emulsification, membrane extrusion, and solvent dispersion methods like ether and ethanol injection and reverse phase evaporation. Lastly, purified liposome product can be analysed. Liposomes are being analysed in order to ensure that they are in the desired shape and size. After this step, liposomes should be kept at the adequate temperature (above the transition temperature which is dependent on lipids used for the production). The preparation of the liposomes can be obtained via detergent solubilization and detergent removal (1.7.4).

Encapsulation of the specific desired molecules (e.g., for drug delivery in human body) can be performed either passively (e.g., the drug is encapsulated during liposome formation) or actively (e.g., after liposome formation).

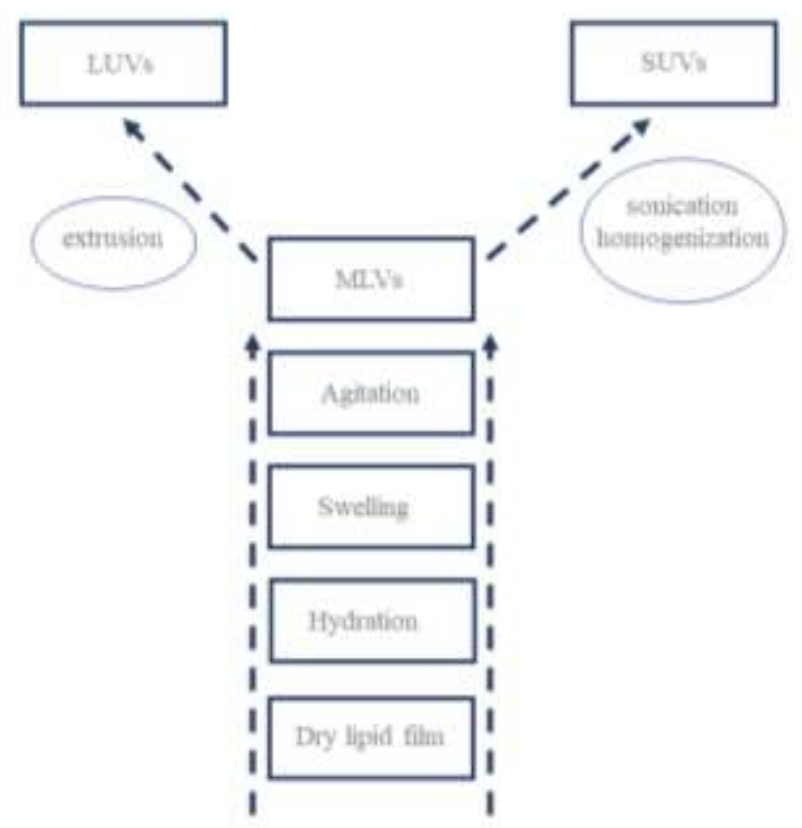

Figure 16. Schematic representation of the steps of the liposome preparation. In chloroform dissolved lipids are dried by removal of the solvent. The resulting lipid film is hydrated and agitated until the formation of MLVs. With the addition of energy to the system (e.g., extrusion or sonication) LUVs or SUVs are formed, respectively.

\subsubsection{Applications of liposomes in medicine and pharmacology}

Advantages of using liposomes make them a desirable tool in medicine and pharmacology. Liposomes can complex both with negatively and positively charged molecules and they can offer some degree of protection to DNA against degradation. Also, liposomes can carry 
molecules as big as the size of a chromosome. Liposomes can be designed to target specific cells and tissues. Liposome-mediated drug delivery systems have been successfully implemented into clinical usage, anti-cancer, anti-fungal and anti-inflammatory treatments. Drug delivery with liposomes is approved for intravenous, intramuscular, oral and spine delivery (Gabizon et al., 1994)(Glantz et al., 1999)(Bovier, 2008) (Bulbake et al., 2017)(He et al., 2019).

For example, liposomes are used in a wide range for the delivery of bactericides. An example is the usage of liposomes to deliver vancomycin in order to treat Staphylococcus aureus, methicillin-resistant bacteria (Onyeji et al., 1994). Ciprofloxacin incapsulated inside the liposomes were used for treatment of rat lung infected with Klebsiella pneumoniae and P. aeruginosa (Bakker-Woudenberg et al., 2001)(Bakker-Woudenberg et al., 2002). Cationic liposomes, when made of amphiphile dioctadecyl dimethylammonium bromide (DODAB) are bactericidal toward E. coli, Salmonella thyphimurium, P. aeruginosa and S. aureus (Campanhã et al., 1999).

A disadvantage of usage of liposomes is that the production of liposomes can be costly. Also, leakage or fusion of the encapsulated molecules can happen. Oxidation and hydrolysis-like reactions, short half-life, and low solubility are also the disadvantages of the liposome treatments, when they are delivered in the human body (Daraee et al., 2016). However, the observed stability and low toxicity made them part of the study in gene therapy (Daraee et al., 2016)(Malone et al., 1989) and drug delivery (Weiner et al., 1989).

\subsubsection{Reconstitution of membrane proteins in lipid vesicles}

When various organisms are analysed, about $25 \%$ of the sequenced genes encode for hydrophobic proteins, that are integrated into the biological membrane (Wallin \& von Heijne, 1998). These proteins are part of many biological processes and it is essential to study and gain knowledge about them. However, the complexity of the membrane does not allow easy investigation. For that reason, extraction of membrane proteins from the membrane and stabilizing them in the solution and then reconstituting into the artificial bilayer is necessary.

In order to imitate the biological membrane, liposomes were widely used. After extensive gain in the biochemical and biophysical knowledge, much more is known about how to 
achieve successful integration of the protein into the liposomes. Four basic methods are used for this purpose: mechanical means, freeze-thawing, organic solvents and detergents (Rigaud \& Lévy, 2003). Important characteristics of the reconstitution product have to be obtained: reconstitution efficiency, homogeneity of the protein insertion, orientation of the protein, size of the final proteoliposomes and possible leakage (permeability).

\subsubsection{Detergent-mediated reconstitution}

Even though there are a number of different methods that can be used for the protein incorporation, the most used is detergent-mediated reconstitution. The other methods are either producing rather small liposomes or damaged liposomes, and membrane proteins are being extracted from the membrane via usage of the detergent, hence it becomes the most logical choice.

It is important to be aware of the fact that reconstitution is a process of several very sensitive steps. First of them is the already mentioned liposomes formation. Next, step by step addition of detergent to the preformed liposomes. The addition of solubilized protein follows. The last but certainly not the least important step is detergent removal (Figure 17, Figure 18). 


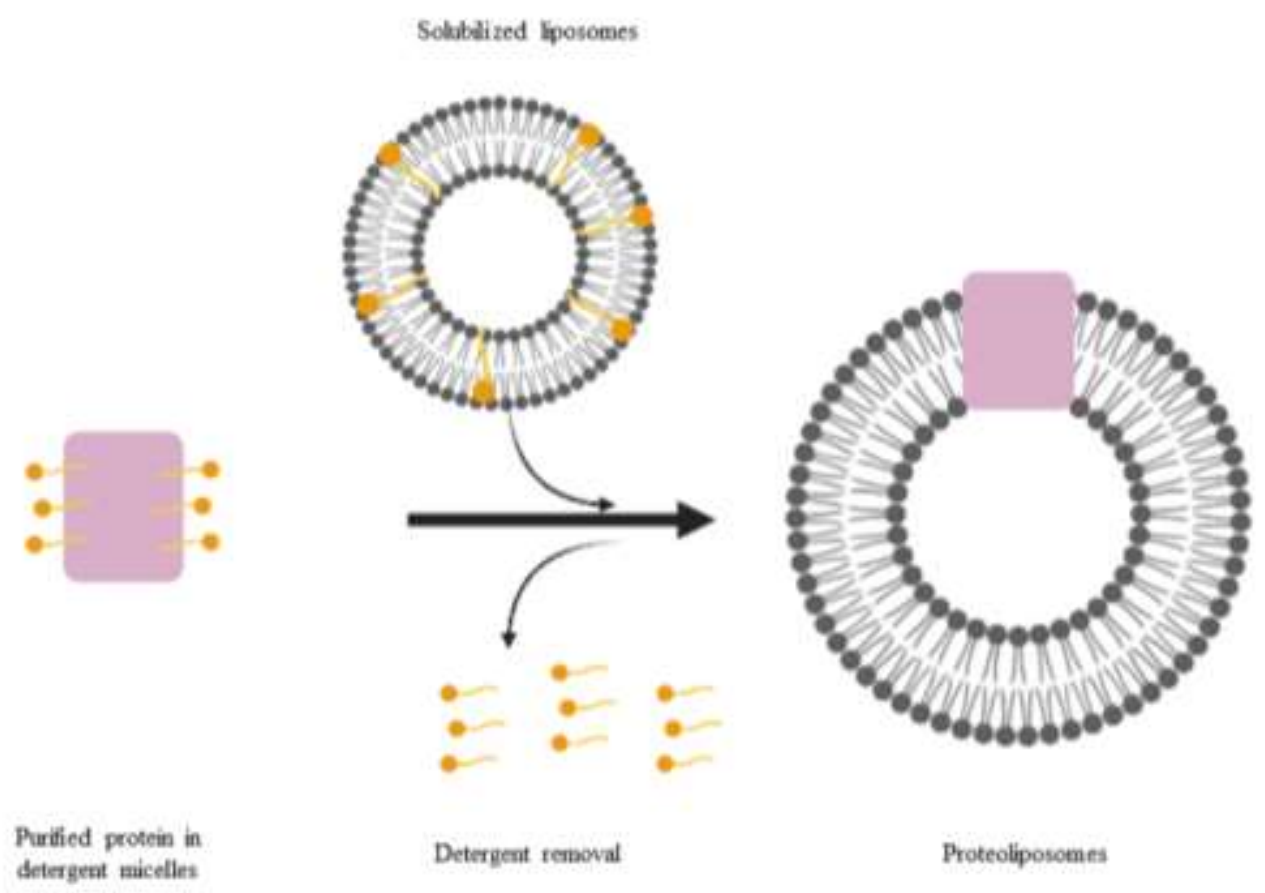

Figure 17. Steps of the detergent-mediated reconstitution. Purified protein is added to the solubilized liposomes. In order for the protein to be incorporated into the bilayer, detergent has to be removed. Figure was made with help of BioRender (https://app.biorender.com/).

Liposome solubilization has several phases. In the phase I, the solution has detergent micelles and liposomes. Phase II is represented with the equilibrium of detergent-saturated liposomes and detergent-phospholipid mixed micelles. Beginning of phase II is introduced as $\mathbf{R}_{\mathrm{sat}}$, marking the onset of solubilization (Geertsma et al., 2008). In phase III, the detergentsaturated liposomes are completely solubilized, and the whole solution contains detergentphospholipids mixed micelles. At the beginning of phase III, $\mathbf{R}_{\text {sol }}$ is introduced, marking total solubilization. This process can be monitor as a change in an absorbance signal of lipids (Rigaud \& Lévy, 2003) (Figure 18). The more solubilized the liposomes, the bigger the decrease of the signal, less turbid the solution.

The problem that can rise during the detergent-mediated reconstitution is the presence of the detergent itself, since it can interfere with the formation of the vesicles. For that reason, it is important to remove the excess amount of detergent. The removal of detergent can be performed via dialysis, gel filtration, dilution and via usage of polystyrene beads. 


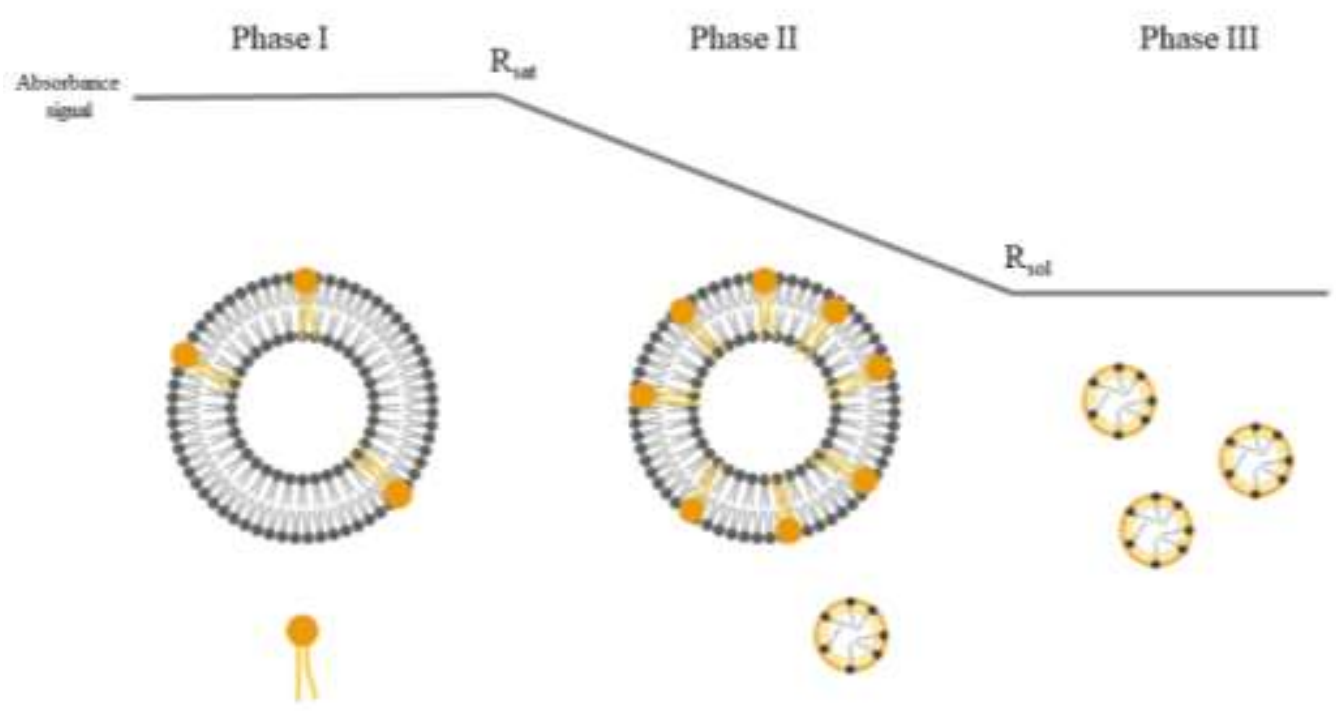

Figure 18. Solubilization of liposomes. Liposome solubilization undergoes three distinct phases. Phase I: In the solution detergent micelles and liposomes are present. Phase II: Detergent-saturated liposomes are in equilibrium with detergentphospholipids mixed micelles, which is accompanied by a decrease of the absorbance signal. Phase III: Solution has detergent-phospholipid mixed micelles. $R_{\text {sat }}$ is a point at the beginning of the phase II, and $R_{\text {sol }}$ at the beginning phase III. Figure was made with help of BioRender (https://app.biorender.com/). Adapted from (Rigaud \& Lévy, 2003).

Dialysis is used for detergent removal and its principle is that detergent monomers diffuse through the dialysis membrane, following their concentration gradient, while large proteoliposomes remain in the sample volume. The rate of the detergent removal depends on the monomer detergent concentration in the sample. Detergent monomers and micelles are in equilibrium and the critical micellar concentration (CMC) is a parameter which differs for all detergents. That is why detergent dialysis is used more conveniently with detergents like octyl-glycoside (high CMC), but less so with dodecyl maltoside (low CMC). CMC is defined as the concentration of surfactants above which micelles are being formed. On the other hand, membrane proteins are more stable and less prone to aggregation in the presence of certain detergents (like DDM) compared to others (like OG). The advantage of the dialysis technique is low cost and homogeneity of the sample. However, it has poor reproducibility, long time for removal, and a not controllable rate of the detergent removed. A nowadays rarely used method is gel filtration, where lipid-protein-detergent micellar mixture is eluted through the resin and results in the formation of vesicles and their extrusion from the pores of the matrix. Even though it is fast, usually it results in non-homogenous samples, with differently sized proteoliposomes. The dilution method is performed with detergent-free buffer which will dilute the liposomes-protein-detergent mixture below the $\mathrm{CMC}$ of the detergent, triggering 
formation of proteoliposomes. This simple technique is fast, and it is possible to monitor the rate of dilution. But not every detergent can be used, only the detergent with high CMCs, additional centrifugation steps are needed and the full detergent removal cannot be done completely with this procedure alone (Rigaud \& Lévy, 2003).

To remove the disadvantages that can happen while detergent removal, like usage of only detergent with high CMC, or the incomplete removal of detergent, another technique is used as a method for proteoliposome formation. The technique uses polystyrene beads that will hydrophobically adsorb detergent onto their resins. The resins used are for example Amberlite XAD or Bio-Beads SM-2. This method is reproducible and its main limitation is that if a surplus of resin is added, lipid-absorption is observed, and this can lead to disruption of proteoliposomes. However, within certain parameters, the resin/beads can completely remove detergent and lead to homogenous and tight proteoliposome samples. This is important for the analysis of the membrane protein transport since it creates samples that do not have high ion permeability (Rigaud \& Lévy, 2003).

\subsubsection{Designing an assay for investigation of RND transporters}

In vivo studies give enormous amount of data and especially the studies done with fluorescent dyes or using minimal inhibitory concentration experiments (MICs; the lowest concentration of an antimicrobial that would inhibit the visible growth of a microorganism) can show immediate effect of the observed protein in the system. The transport mechanism and substrate specificity as well as kinetic constants of the transporter can be examined in the cell, as well. The advantage of liposome usage is the possibility to uncouple the metabolism factor from the complex system as cell is. There is no breakdown or modification of the transported compound and for example, in case of purified transporters, they can be studied without the presence of other transporters (that would be present in the cell) which might have the same, or opposite (influx vs. efflux) effects.

Designing an assay for studying membrane proteins is a challenge. The protein has to be extracted from its native environment and transferred into the new, artificial membrane. Usually, liposomes are used as a host environment that mimics the native environment. The reconstitution of a transport protein allows vectorial substrate transport observations. When 
used with a specific purified membrane protein sample, transport activity can be monitored in absence of the complex cell membrane system.

The first assay in vitro involving membrane protein AcrB reconstituted in liposome, was done by Zgurskaya and Nikaido in 1999 (H. I. Zgurskaya \& Nikaido, 1999). The principle of the assay was that $\mathrm{AcrB}$ proteoliposomes containing $\mathrm{KCl}$ and pyranine (as $\mathrm{pH}$ sensor) were diluted into a buffer containing $\mathrm{NaCl}$. Then valinomycin was added in order to facilitate the movement of potassium ions through the lipid membrane (down the chemical gradient of potassium ions). The electrical potential created by the $\mathrm{K}^{+}$-ions led to proton influx due to the apparently $\mathrm{H}^{+}$permeable proteoliposomes and a $\Delta \mathrm{pH}$ (inside acid) was established, observed via the quenching of the pyranine fluorescence. Since AcrB is a $\mathrm{H}^{+} / \mathrm{drug}$ antiporter, the outward directed $\mathrm{pH}$ gradient was utilized in antiport fashion by AcrB in the presence of substrate, leading to the dequenching of the pyranine fluorescent signal.

Except using Valinomycin, the same was achieved by using a $\mathrm{pH}$ gradient, where AcrB proteoliposomes were diluted in acidic buffer. The $\mathrm{pH}$ inside the liposome was monitored using pyranine that was encapsulated in the liposomes. The fluorescence of entrapped pyranine in AcrB proteoliposomes was measured over time. When substrates of AcrB are then added and a $\Delta \mathrm{pH}$ (inside acidic) was applied over the membrane, the proton efflux was observed (Figure 19).

Another assay for showing the activity of AcrB was performed in the same study. In the presence of a $\Delta \mathrm{pH}$ (inside alkaline) fluorescent phospholipids (NBD- and rhodamine-labelled phospholipids) present in AcrB proteoliposomes were trapped by protein-free acceptor vesicles. In the presence of known substrates of AcrB, the efflux of fluorescent phospholipid was inhibited. The AcrB activity was enhanced by the addition of AcrA and also by addition of $\mathrm{Mg}^{2+}$. 


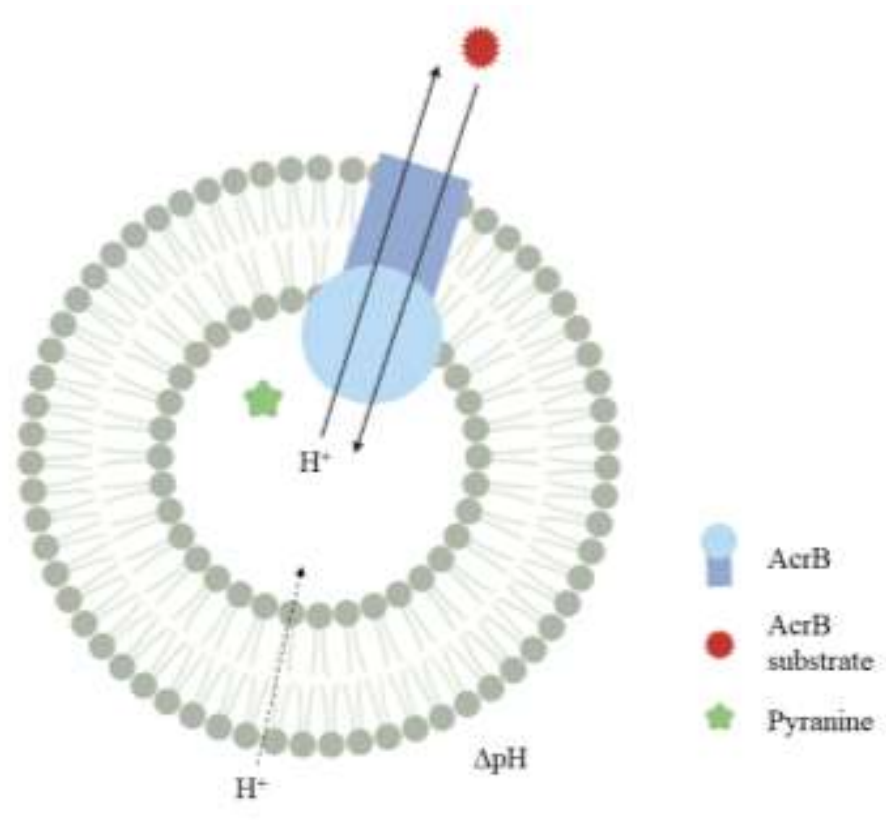

Figure 19. AcrB proteoliposomes assay. Schematic representation of AcrB proteoliposomes in the presence of a proton gradient, together with a substrate and a $\mathrm{pH}$ reporter, pyranine. AcrB is depicted in the inside-out orientation. The electrical gradient is converted in a $\mathrm{pH}$ gradient which then is used by AcrB in an antiport fashion to take up substrates. The $\mathrm{pH}$ gradient was obtained from an electrical gradient that is formed upon addition of valinomycin. The readout in this experiment is proton transport. Figure was made with help of BioRender (https://app.biorender.com/).

Another RND efflux pump that has been tested in vitro was MexB (Welch et al., 2010). MexB proteoliposomes contained DNA. To impose diffusion gradients, proteoliposomes contained potassium phosphate buffer $\mathrm{pH} \mathrm{7,} \mathrm{potassium} \mathrm{acetate} \mathrm{and} \mathrm{valinomycin} \mathrm{(to} \mathrm{prevent}$ the formation of a reverse $\Delta \psi$ ) were diluted into the same buffer (no gradient) or into potassium phosphate buffer $\mathrm{pH} 7$ containing $\mathrm{K}_{2} \mathrm{SO}_{4}$ to generate an acetic acid diffusioninduced $\Delta \mathrm{pH}$ (interior alkaline). Then Hoechst 33342 dye was added, which is a substrate of the pump. This dye is intercalating with DNA and results in an enhanced quantum yield and in the increase of the fluorescence. The fluorescence increase was observed when Hoechst 33342 intercalates with DNA after spontaneous diffusion of the dye across the lipid bilayer (when no active MexB is present or when the inactive protein, MexB D407N, is being reconstituted in liposomes). Hoechst 33342 is being transported out of the liposomes, resulting in a slow quench of fluorescent signal when active MexB is reconstituted in liposomes.

When CusA was reconstituted in liposomes (Long et al., 2010) and a stop flow assay was performed, it was shown that this RND protein has an active transport of $\mathrm{Ag}^{+}$from the cytoplasmic side of the protein. The reporter dye (PGSK) used in this study, binds metal ions 
and binding of silver ions decreases the fluorescent signal. PGSK was present inside the liposomes, and silver, a substrate of the pump, was present in the outside buffer. Upon imposition of a $\Delta \mathrm{pH}$ (inside acidic) the $\mathrm{H}^{+} / \mathrm{Ag}^{+}$antiport resulted in the transport of $\mathrm{Ag}^{+}$to the inside of the proteoliposomes observed by the fluorescent quenching of PGSK. When the CusA mutants (variants having mutations in the residues belonging to the proton relay network, or to the methionine ( $\mathrm{Ag}^{+}$-transport) pathway) were used, the protein did not show activity.

The first in vitro assay of the whole tripartite system was done by Verchère et al in 2015, when the MexAB-OprM pump was analysed (Verchère et al., 2015). The principle of the assay is based on two batches of proteoliposomes, one into which OprM was reconstituted, the other into which MexAB were reconstituted. The OprM proteoliposomes contained RNA so that, once ethidium is transported into the OprM liposomes, it binds to the RNA and the fluorescence increases. When ethidium bromide is added to the outside medium, and the pump is assembled and active, upon the imposition of a $\Delta \mathrm{pH}$ (inside alkaline), ethidium is transported via MexB into the OprM vesicle where it interacts with RNA (Figure 20).

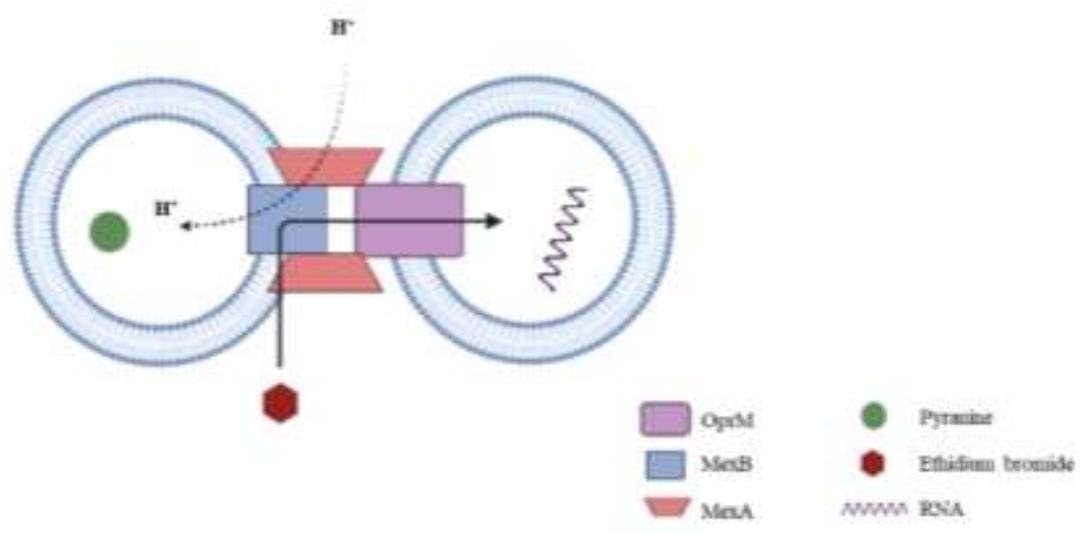

Figure 20. Schematic representation of the MexAB-OprM in vitro assay. MexB transports its substrate, ethidium bromide (EthB), to the TolC proteoliposome, where it would interact with RNA. Increase of the EthB fluorescence would be measured. On the other hand, together with the substrate transport, protons are being transported into MexB proteoliposome, where pyranine fluorescence would be quenched upon acidification of the lumen. Figure was made with help of BioRender (https://app.biorender.com/). Adapted from (Verchère et al., 2015).

At the same time, protons are being transported through MexB into the MexAB proteoliposomes where they quench the incapsulated dye, pyranine, by acidification of the liposome interior. $\mathrm{H}^{+}$-transport into the MexAB proteoliposomes was also observed in the presence of substrate meropenem. This activity was enhanced when OprM proteoliposomes 
were added to MexAB proteoliposomes. When the inactive variant of MexB was reconstituted with MexA $\left(\operatorname{Mex} \mathrm{B}_{\mathrm{D} 407 \mathrm{~N}}\right)$ or when empty liposomes were used, the pyranine fluorescent signal was stable, indicating that the proton transport does not take place.

As a complementary approach to the study of the MexAB-OprM activity in proteoliposomes (Verchère et al., 2015) a new strategy was presented that allowed investigation of the formation of efflux pumps (Ntsogo Enguéné et al., 2015). Usage of streptavidin-coated magnetic beads to specifically sediment MexAB proteoliposomes containing biotinylated lipids was used in order to examine the formation of the complex. When MexAB proteoliposomes were preincubated with OprM proteoliposomes and pulled down via biotinylated lipids in the MexAB proteoliposomes, all three proteins (MexA, MexB and OprM) were detected on the SDS-PAA gel, indicating that OprM proteoliposomes were coprecipitated. A complex was also formed when the inactive D407N variant of MexB was used. However, when MexB was reconstituted with non-membrane-anchored, soluble MexA or was reconstituted alone, no co-precipitation of OprM proteoliposomes was detected. The pull-down assay was also performed under the conditions of a transport assay, with applying $\Delta \mathrm{pH}$. For the actual activity observation of the assembled complex, pyranine and ethidium fluorescence were monitored. The MexAB-OprM complex formation was detected when $\Delta \mathrm{pH}$ was applied, at $\mathrm{t}=0$ minutes, but the complex was not detected after five minutes, $\mathrm{t}=5$ minutes. 


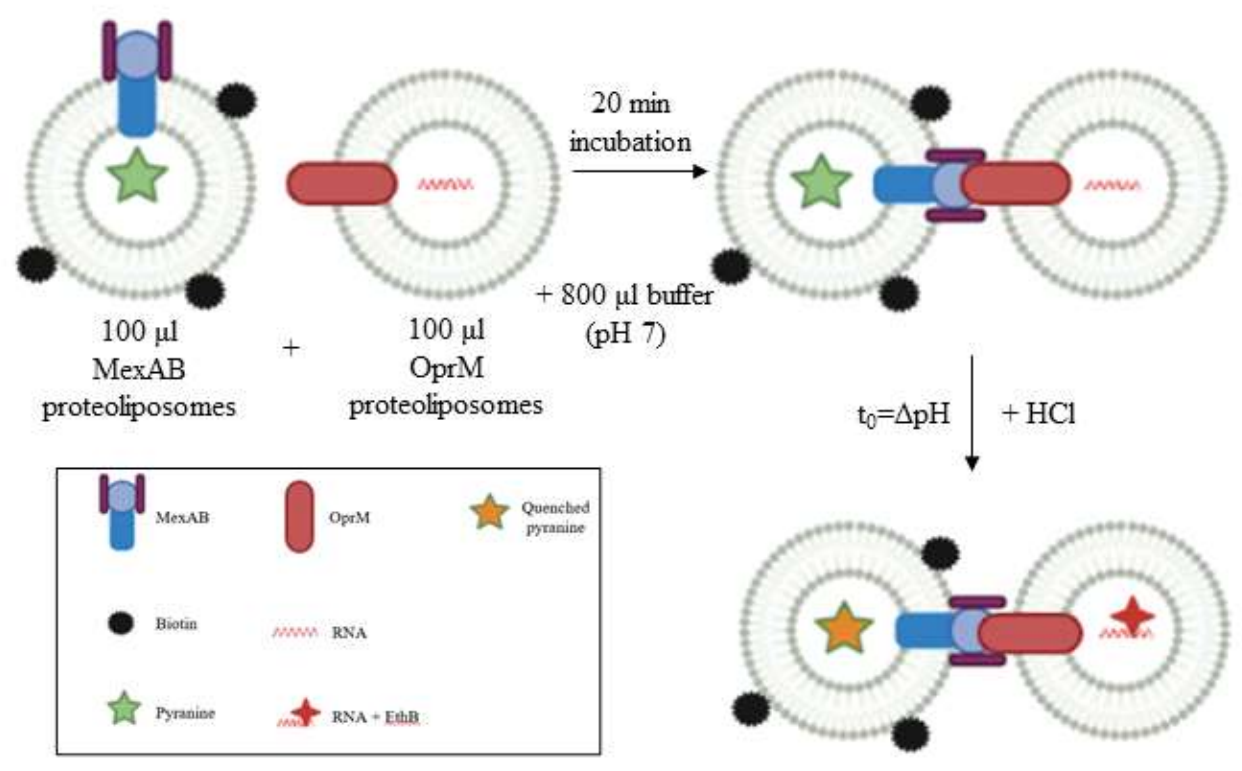

Figure 21. Schematic representation of the pull-down experiment with MexAB and OprM proteoliposomes. The proteoliposomes (MexAB and OprM) were mixed and incubated with EthB. A pH gradient was applied after 20 minutes incubation by addition of hydrochloric acid $(\mathrm{HCl})$. Mex $\mathrm{AB}$ proteoliposomes have a biotin-cap and pyranine incapsulated. The pull-down was observed on SDS-PAA gel and the activity of the pump was monitored with dual wavelength fluorescence spectroscopy. Figure was made with help of BioRender (https://app.biorender.com/). Adapted from (Ntsogo Enguéné et al., 2015).

However, MexAB D407N-OprM complex was detected both at $\mathrm{t}=0$ and $\mathrm{t}=5$ minutes, which was an indication that the efflux pump assembly does not require transport activity. These are two indications for the idea that the proton motive force promotes disassembly of the complex via $\mathrm{H}^{+}$-transport active MexB; first, the disappearing of OprM when the wild type pump has reached its steady state (as detected at $\mathrm{t}=5$ minutes) and second, by the maintaining of the assembly when the mutant defective in proton translocation, D407N, was used (Figure 21) (Ntsogo Enguéné et al., 2015).

These described assays are part of the remarkable accomplishments to understand how substrate transport works when RND transporters are involved. And even with this substantial knowledge that is collected over the years, in vitro analysis of $\mathrm{H}^{+}$and drug transport by tripartite systems is still awaiting more stable systems to measure transport kinetics. We need a universal, reproducible in vitro system, that can help us investigate tripartite multidrug efflux systems in order to understand transport and mechanisms of substrate translocation, but it also can help with the design of new, improved inhibitor classes. 


\section{Chapter II: Aim of the thesis}

The members of the Resistance Nodulation cell Division (RND) superfamily in Gramnegative bacteria play an important role in establishing resistance phenotypes. These inner membrane proteins interact with periplasmic membrane fusion proteins (MFPs), and the RND-MFP bipartite complexes recruit outer membrane proteins (OMPs). This threecomponent RND-MFP-OMP formation spans the Gram-negative inner and outer membranes and is in charge of expelling toxic compounds from both cytoplasm and periplasm. Many of the Gram-negative RND protein complexes are involved in the extrusion of antibiotics, but some members of this superfamily expel heavy metal ions.

Even though nowadays the structures of the individual components and of the entire three partite complexes of these pumps are known, some questions still remain unanswered. Which are the factors that allow or inhibit the complex formation? What is the behaviour of the pump in the presence of different inhibitors? What are the pathways of substrate transport within the RND component of the pump complex? What are ways to study these pumps in vitro? The current work tries to answer some of those questions while performing a functional description of the efflux pumps in vitro of the clinically relevant bacteria, E. coli.

In order to answer these questions, the work was focusing on: Overexpression and purification of the single drug efflux system components, reconstitution of these components in liposomes, establishing a two-batch proteoliposomal system mimicking the in vivo inner and outer membrane setup, analysis of the $\mathrm{H}^{+}$- and metal ion fluxes in the presence and absence of active drug/metal-ion transporters, and drug susceptibility assays.

Looking at the big picture, this study contributes to the better understanding of multicomponent efflux pumps and their assembly. This work provides the design for in vitro assays that in the future can be used for the determination of the kinetics of drug transport and may help the search and characterization of new potent efflux pump inhibitors. 


\section{Chapter III: Material and Methods}

\subsection{Material}

\subsubsection{Laboratory equipment}

Table 1. Laboratory equipment used in the thesis.

\begin{tabular}{|c|c|}
\hline Type of equipment & Name \\
\hline Centrifuges & $\begin{array}{l}\text { Sorvall Evolution RC (Thermo Scientific) } \\
\text { Sorvall WX Ultra Series (Thermo Scientific) } \\
\text { Optima XPN-100 (Beckman Coulter) } \\
\text { Optima TLX-120 (Beckman Coulter) } \\
\text { Heraeus Biofuge } 13 \text { (Heraeus) } \\
\text { Heraeus Multifuge 1S-R (Thermo Scientific) }\end{array}$ \\
\hline Rotors & $\begin{array}{l}\text { FiberLite F8-6x1000y (Thermo Scientific) } \\
\text { FiberLite F9-6x1000LEX (Thermo Scientific) } \\
\text { FiberLite F14-6x250y (Thermo Scientific) } \\
\text { Beckman Ti45 (Beckman Coulter) } \\
\text { Beckman Ti60 (Beckman Coulter) } \\
\text { Beckman TLA100 (Beckman Coulter) } \\
\text { Beckman TLX120 (Beckman Coulter) Heraeus Swing-out TTH } 400 \text { (Thermo } \\
\text { Scientific) }\end{array}$ \\
\hline Incubators & $\begin{array}{l}\text { Plate incubator (Heraeus Instrument) } \\
\text { HT Multitron (Infors) } \\
\text { C24 Incubator Shaker (New Brunswick Scientific) }\end{array}$ \\
\hline Imagers & $\begin{array}{l}\text { ImageQuant LAS4000 (GE Healthcare) } \\
\text { Intas Gel Imager (Intas) }\end{array}$ \\
\hline Spectroscopy & $\begin{array}{l}\text { Tecan Reader Infinite M200 (Tecan AG) Nanodrop spectrophotometer ND- } \\
1000 \\
\text { (Peqlab Biotechnologie GmbH) } \\
\text { Cell density meter Ultrospec } 10 \text { (Amersham Biosciences) } \\
\text { Cary } 50 \text { UV-Vis spectrophotometer (Varian Inc) } \\
\text { Jobin Yvon Fluorolog } 3 \text { (Horiba Scientific) } \\
\text { Fluorescence Spectrometer Cary Eclipse (Varian) }\end{array}$ \\
\hline Chromatography & Äkta Purifier (GE Healthcare) \\
\hline Various & $\begin{array}{l}\text { Mastercycler epgradient S (Eppendorf) Thermomixer comfort (Eppendorf) } \\
\text { Homogenizer RZR-1 (Heidolph) }\end{array}$ \\
\hline
\end{tabular}




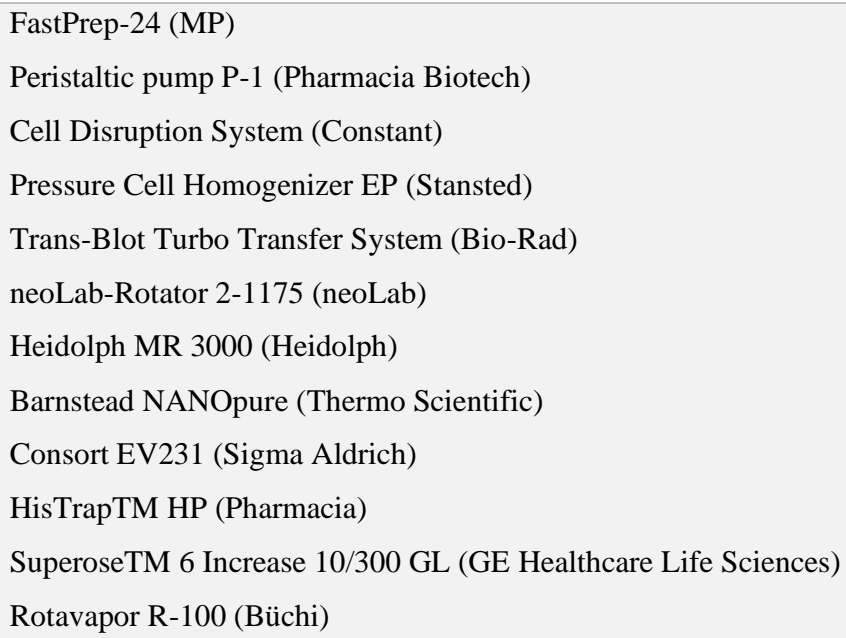

\subsubsection{Chemicals}

Chemicals were obtained from: Bio-Rad (Hercules, USA), NeoLab (Heidelberg, Germany), Sigma-Aldrich (ST. Louis, MO, USA), Carl Roth (Karlsruhe, Germany), Merck (Darmstadt, Germany), Avanti Polar Lipids (Birmingham, Alabama, USA), Thermo Fisher Scientific (Waltham, USA), Invitrogen (Carlsbad, USA), Applichem (Darmstadt, Germany), Glycon (Luckenwalde, Germany) or Life Technologies (Darmstadt, Germany), unless stated otherwise. 


\subsubsection{Bacterial strains: for molecular biology techniques, for protein production, for functional assay}

Table 2. Bacterial strains used in the thesis.

\begin{tabular}{|c|c|c|}
\hline Name & Description & Source \\
\hline $\begin{array}{l}\text { E. coli } \mathrm{MC1061} \\
\text { (FX cloning) }\end{array}$ & 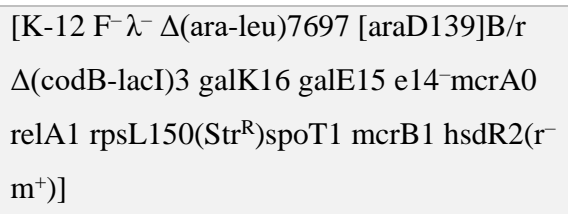 & $\begin{array}{l}\text { Lab collection } \\
\text { (Casadaban \& Cohen, } \\
\text { 1980) }\end{array}$ \\
\hline $\begin{array}{l}\text { E. coli } \mathrm{C} 43 \text { (DE3) } \triangle a c r A B \\
\text { (protein production) }\end{array}$ & $\begin{array}{l}{\left[\mathrm{F}^{-} \text {ompT hsdS } \mathrm{B}_{\mathrm{B}}\left(\mathrm{r}_{\mathrm{B}^{-}} \mathrm{m}_{\mathrm{B}}^{-}\right) \text {gal dcm (DE3)] }\right.} \\
\Delta \text { acrAB }\end{array}$ & $\begin{array}{l}\text { Lab collection } \\
\text { (Miroux \& Walker, } \\
\text { 1996) }\end{array}$ \\
\hline $\begin{array}{l}\text { E. coli } \mathrm{C} 43 \text { (DE3) } \triangle a c r A B D \\
\text { (protein production) }\end{array}$ & $\begin{array}{l}{\left[\mathrm{F}^{-} \text {ompT hsdS } \mathrm{B}_{\mathrm{B}}\left(\mathrm{rB}^{-} \mathrm{mB}^{-}\right) \text {gal dem (DE3)] }\right.} \\
\Delta \text { acrAB } \Delta \mathrm{acrD}\end{array}$ & $\begin{array}{l}\text { Lab collection } \\
\text { (Miroux \& Walker, } \\
\text { 1996) (T. Eicher et al., } \\
\text { 2012) } \\
\text { (as a courtesy of Dr. } \\
\text { Viveka Malviya Nand) }\end{array}$ \\
\hline $\begin{array}{l}\text { E. coli BW25113 (DE3) } \\
\triangle a c r A B \\
\text { (functional assay) }\end{array}$ & 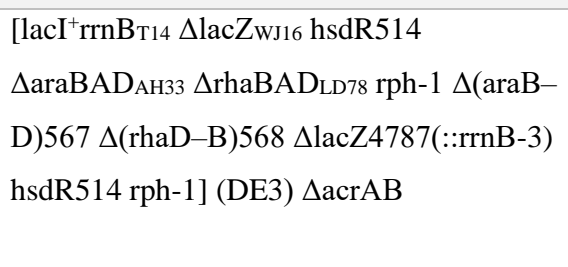 & $\begin{array}{l}\text { Lab collection } \\
\text { (Datsenko \& Wanner, } \\
2000) \\
\text { (Grenier et al., 2014) } \\
\text { (T. Eicher et al., 2012) }\end{array}$ \\
\hline $\begin{array}{l}\text { E. coli BW25113 } \\
\text { (functional assay) }\end{array}$ & $\begin{array}{l}{\left[\text { lacI }^{+} \mathrm{rrnB}_{\mathrm{T} 14} \Delta \mathrm{lacZ}_{\mathrm{WJ16}} \text { hsdR514 }\right.} \\
\Delta \operatorname{araBAD}_{\mathrm{AH} 33} \Delta \text { rhaBAD }{ }_{\text {LD78 }} \text { rph-1 } \Delta(\mathrm{araB}- \\
\text { D)567 } \Delta(\mathrm{rhaD}-\mathrm{B}) 568 \Delta \mathrm{lacZ} 4787(:: \mathrm{rrnB}-3) \\
\text { hsdR514 rph-1] }\end{array}$ & $\begin{array}{l}\text { Lab collection } \\
\text { (Datsenko \& Wanner, } \\
2000) \\
\text { (Grenier et al., 2014) }\end{array}$ \\
\hline $\begin{array}{l}\text { E. coli C41 (DE3) } \\
\text { (functional assay) }\end{array}$ & {$\left[\mathrm{F}^{-}\right.$ompT gal dcm hsdS $\left.\mathrm{B}_{\mathrm{B}}\left(\mathrm{r}^{-} \mathrm{mB}^{-}\right)(\mathrm{DE} 3)\right]$} & $\begin{array}{l}\text { (Miroux \& Walker, } \\
\text { 1996) }\end{array}$ \\
\hline $\begin{array}{l}\text { E. coli C41 (DE3) } \triangle a c r A B \\
\text { (functional assay) }\end{array}$ & $\begin{array}{l}{\left[\mathrm{F}^{-} \text {ompT gal dcm hsdS } \mathrm{S}_{\mathrm{B}}\left(\mathrm{r}_{\mathrm{B}^{-}} \mathrm{m}^{-}\right)(\mathrm{DE} 3)\right]} \\
\Delta \mathrm{acrAB}\end{array}$ & $\begin{array}{l}\text { Lab collection } \\
\text { (Miroux \& Walker, } \\
\text { 1996) }\end{array}$ \\
\hline
\end{tabular}

Genomic DNA E. coli BL21 (DE3) was used (lab collection). 


\subsubsection{Plasmids}

Table 3. Plasmids used in the thesis.

\begin{tabular}{|c|c|c|}
\hline Plasmid & Description & Gene fused tags \\
\hline \multicolumn{3}{|l|}{ Plasmids used for FX cloning } \\
\hline pINITcat (Geertsma \& Dutzler, 2011) & $\mathrm{Cam}^{\mathrm{r}}$ & \\
\hline P7XC3H (Geertsma \& Dutzler, 2011) & $\begin{array}{l}\text { T7 promoter } \\
\operatorname{Kan}^{\mathrm{r}}\end{array}$ & $\begin{array}{l}\text { C-terminal: HRV3C protease cleavage } \\
\text { recognition site } 10 x H i s \text { tag }\end{array}$ \\
\hline \multicolumn{3}{|l|}{ Plasmids used for functional studies } \\
\hline pUC19 (Norrander et al., 1983) & $\begin{array}{l}\text { T7 promoter } \\
\text { Amp }\end{array}$ & \\
\hline pET24a (Novagen) & $\begin{array}{l}\text { T7 promoter } \\
\operatorname{Kan}^{\mathrm{r}}\end{array}$ & C-terminal: $6 x$ His tag \\
\hline
\end{tabular}

Constructs: p7XC3RH_emrB and pRSFDMG_emrA_tolC, provided with courtesy by Dr. Narek Yousefian.

\subsubsection{Primers}

All primers were obtained from Sigma Aldrich.

Table 4. Primers used for FX cloning.

\begin{tabular}{|l|l|}
\hline Primer & Sequence $\left(\mathbf{5}^{\prime} \rightarrow \mathbf{3}^{\prime}\right)$ \\
\hline FW acrA_A200V & ATATATGCTCTTCTAGTGACGACAAACAGGCCCAACAAGG \\
\hline RV acrA_A200V & TATATAGCTCTTCATGCAGACTTGGACTGTTCAGGCTGAGC \\
\hline FW copA & ATATATGCTCTTCTAGTTCACAAACTATCGACCTGA \\
\hline RV copA & TATATAGCTCTTCATGCTTCCTTCGGTTTAAACCGC \\
\hline FW cusA & ATATATGCTCTTCTAGTATTGAATGGATTATTCGTC \\
\hline RV cusA & TATATAGCTCTTCATGCTTTCCGTACCCGATGTCGG \\
\hline FW cusA_D405N & AATGCTGCTATCGTCATGA \\
\hline RV cusA_D405N & GACCATCGCCCCGACGGCA \\
\hline
\end{tabular}


Table 5. Primers used for sequencing.

\begin{tabular}{|l|l|}
\hline Primer & Sequence $\left(5^{\prime} \rightarrow 3^{\prime}\right)$ \\
\hline pINITcat $\mathbf{F W}$ & GAGTAGGACAAATCCGCCGC \\
\hline pINITcat $\mathbf{R V}$ & CAACGTTCAAATCCGCTCCC \\
\hline $\mathbf{p 7 x} \mathbf{~ F W}$ & ACCTGTCCTACGAGTTGCATG \\
\hline $\mathbf{p 7 x} \mathbf{R V}$ & CTTCTGAGATGAGTTTTTG \\
\hline $\mathbf{T 7}$ & TAATACGACTCACTATAGGG \\
\hline T7 term & TGCTAGTTATTGCTCAGCGG \\
\hline copA mid & AGCCTGTGGTTGGTTATCGG \\
\hline cusA $\mathbf{1}$ & GGTTGTGTATTGCTTTTATTGTCATGCACTTCCAGG \\
\hline cusA 2 & AAGGTCCAGATTGGCCCGGAGATGCG \\
\hline
\end{tabular}

\subsubsection{Enzymes}

All restriction enzymes, DNA polymerases and DNA modifying enzymes were obtained from Thermo Fisher Scientific (Waltham, MA, USA, former Fermentas).

\subsubsection{Kits}

Table 6. Kits used in the thesis.

\begin{tabular}{|l|l|}
\hline Name & Company \\
\hline Mini Prep QIAPrep Spin Miniprep Kit & Qiagen, Venlo, Netherlands \\
\hline DNA Clean \& Concentrator ${ }^{\text {TM-5 Kit }}$ & Zymo Research, Irvine, CA, USA \\
\hline GeneRulerTM 1kb DNA ladder & Thermo Fisher Scientific, Waltham, MA, USA \\
\hline PageRulerTM protein ladder & Thermo Fisher Scientific, Waltham, MA, USA \\
\hline Zymoclean Gel DNA Recovery Kit & Zymo Research, Irvine, CA, USA \\
\hline
\end{tabular}




\subsubsection{Antibiotics}

Table 7. Antibiotics used in the thesis.

\begin{tabular}{|l|l|l|}
\hline Antibiotic & Stock concentration $(\mathbf{m g} / \mathbf{m l})$ & Solvent \\
\hline Ampicillin (Amp) & 100 & Water \\
\hline Kanamycin (Km) & 50 & Water \\
\hline Chloramphenicol (CAT) & 34 & Ethanol \\
\hline Carbenicillin (CARB) & 50 & water \\
\hline
\end{tabular}

\subsubsection{Media}

Table 8. Media used in the thesis.

\begin{tabular}{|l|l|}
\hline Media & Composition (1 l) \\
\hline LB (Lysogeny Broth) & $10 \mathrm{~g}$ Tryptone \\
& $5 \mathrm{~g}$ Yeast extract \\
& $10 \mathrm{~g} \mathrm{NaCl}$ \\
& For LB-agar plates: 16 g agar \\
\hline TB (Terrific Broth) & $12 \mathrm{~g}$ Tryptone \\
& $24 \mathrm{~g}$ Yeast extract \\
& $4 \mathrm{~mL}$ Glycerol \\
& $0.017 \mathrm{M} \mathrm{KH} \mathrm{PO}_{4}$ \\
& $0.072 \mathrm{M} \mathrm{K}_{2} \mathrm{HPO}_{4}$ \\
\hline MH (Müller-Hinton) Agar & $300 \mathrm{~g}$ Dehydrated infusion from beef \\
& $17.5 \mathrm{~g}$ Casein hydrolysate \\
& $1.5 \mathrm{~g}$ Starch \\
& $17 \mathrm{~g}$ Agar \\
\hline
\end{tabular}

\subsubsection{Lipids}

All the lipids used are purchased from Avanti Polar Lipid, Birmingham, Alabama, USA or Sigma Aldrich, Merck, Darmstadt, Germany, if not stated otherwise. 


\subsection{Methods}

\subsubsection{Molecular biology methods}

\subsubsection{Preparation of chemically competent $E$. coli cells}

Adequate E. coli strains were chosen for the needs of the experiments and the cells of the strains were streaked out on LB agar plate (lab glycerol stocks). Single clone from the plate was picked after overnight incubation at $37^{\circ} \mathrm{C}$, and inoculated into $5 \mathrm{ml} \mathrm{LB}$ medium for overnight growth. The preculture was grown at $37{ }^{\circ} \mathrm{C}$ under constant shaking. $300 \mu$ of the culture were transferred into $100 \mathrm{ml}$ fresh LB medium and cells were cultured at $37{ }^{\circ} \mathrm{C}$ up to an $\mathrm{OD}_{600}$ of 0.5-0.6. Importantly, all following steps were performed at $4{ }^{\circ} \mathrm{C}$. Cells were then harvested by centrifugation (10 minutes, $3000 \mathrm{~g}$ in Swing-out TTH 400 rotor (Heraeus)). The resulting pellet was resuspended in $10 \mathrm{ml}$ buffer $(60 \mathrm{mM} \mathrm{CaCl} 2+15 \%$ Glycerol $)$ and incubated for 1 hour on ice. Next, cells were centrifuged for 10 minutes at $3000 \mathrm{~g}$ in a Swingout TTH 400 rotor (Heraeus). After removal of the supernatant, cells were resuspended in 2 $\mathrm{ml}$ of the same buffer as stated above and kept on ice for additional 1 hour. 50-100 $\mu 1$ aliquots were frozen in liquid nitrogen and stored at $-80{ }^{\circ} \mathrm{C}$.

\subsubsection{Preparation of electrocompetent $E$. coli cells}

E. coli strains were streaked on LB agar plates and incubated overnight at $37{ }^{\circ} \mathrm{C}$. A single colony was inoculated into $10 \mathrm{ml}$ fresh LB-medium and cultivated. The next day, 0.51 fresh LB medium was mixed with $2.5 \mathrm{ml}$ of the preculture and cells were grown at $37{ }^{\circ} \mathrm{C}$ to an $\mathrm{OD}_{600}$ of 0.4 . Following steps were done at $4{ }^{\circ} \mathrm{C}$. The culture was kept on ice for 20 minutes, and then centrifuged for 20 minutes at $1000 \mathrm{~g}$ in a FiberLite F8-6x1000y rotor (Thermo Scientific). The pellet was resuspended in $50 \mathrm{ml}$ ice cold MilliQ H2O, then centrifuged for 15 minutes at $2000 \mathrm{~g}$ in a Swing-out TTH 400 rotor (Heraeus). Afterwards, when pellet was resuspended in $10 \%$ Glycerol, it was centrifuged again, for 15 minutes at $2000 \mathrm{~g}$ in a Swing- 
out TTH 400 rotor (Heraeus). Cells were resuspended in $10 \%$ Glycerol. $100 \mu$ l aliquots were prepared, frozen in liquid nitrogen and stored at $-80{ }^{\circ} \mathrm{C}$.

\subsubsection{Transformation of chemically competent $E$. coli cells}

Cells from the chosen $E$. coli strains were transformed by mixing $50 \mu$ l of chemically competent cells with the adequate plasmid in an amount of around $100 \mathrm{ng}$. The mix was incubated on ice for 20 minutes, and then the heat shock method was used - sample was incubated at $42{ }^{\circ} \mathrm{C}$ for $45-60$ seconds and placed back on ice for 2 minutes. $950 \mu \mathrm{l}$ of LB medium were added to the mixture and the cells were incubated at $37{ }^{\circ} \mathrm{C}$ for 1 hour under constant shaking. The cells were pelleted by centrifugation at $2000 \mathrm{~g}$ for 5 minutes, resuspended in 100-200 $\mu \mathrm{LB}$ medium and plated on LB agar plate containing the appropriate antibiotic. The plate was incubated at $37^{\circ} \mathrm{C}$ overnight.

\subsubsection{Transformation of electrocompetent $E$. coli cells}

Electrocompetent E. coli cells were mixed with desired plasmid, $100 \mu \mathrm{l}$ cells with $50 \mathrm{ng}$ plasmid. For co-transformation, $50 \mathrm{ng}$ of the second plasmid was added to the mix. Electroporation was done with a single pulse in $2 \mathrm{~mm}$ gap electroporation cuvette at $2000 \mathrm{~V}$. Mix was then quickly transferred to the prewarmed $5 \mathrm{ml} \mathrm{LB}$ medium, supplemented with $0.5 \%$ glucose and then incubated at $37{ }^{\circ} \mathrm{C}$ for 1 hour. Cells were then pelleted at $3000 \mathrm{~g}$ for 10 minutes in Swing-out TTH 400 rotor (Heraeus), the cell pellet was resuspended in $100 \mu \mathrm{l}$ LB medium and the cells were plated on LB agar plate supplemented with the respective antibiotic(s), $50 \mu \mathrm{g} / \mathrm{ml}$ Kanamycin alone or together with $50 \mu \mathrm{g} / \mathrm{ml}$ Carbenicillin. 


\subsubsection{Polymerase chain reaction (PCR)}

In order to perform FX cloning (3.2.1.9), amplification of DNA was performed via polymerase chain reaction (PCR). Reaction mixture and PCR program are shown in Table 9, Table 10 and Table 11, respectively.

Table 9. Polymerase Chain Reaction (PCR) mix.

\begin{tabular}{|l|l|}
\hline & Final concentration \\
\hline autoclaved ddH $\mathbf{H}_{\mathbf{2}} \mathbf{O}$ & Filled up until $50 \mu \mathrm{l}$ \\
\hline $\mathbf{5 X}$ Phusion GC buffer & $1 \mathrm{X}$ \\
\hline dNTPs $(\mathbf{1 0} \mathbf{~ m M})$ & $0.25 \mathrm{mM}$ \\
\hline fw primer $(\mathbf{1 0} \boldsymbol{\mu M})$ & $0.5 \mu \mathrm{M}$ \\
\hline rv primer $(\mathbf{1 0} \boldsymbol{\mu M})$ & $0.5 \mu \mathrm{M}$ \\
\hline genomic DNA & $50 \mathrm{ng}$ \\
\hline Phusion DNA polymerase & $1.0 \mathrm{units} / 50 \mu 1 \mathrm{PCR}$ \\
\hline
\end{tabular}

Table 10. PCR program for DNA amplification: denaturation (I step); denaturation, annealing, extension (II step) and final extension (III step). This program uses a touchdown PCR approach (*) where the temperature is decreasing from $65^{\circ} \mathrm{C}$ to $50{ }^{\circ} \mathrm{C}$ by $0.5^{\circ} \mathrm{C}$ in the annealing step (step II) of every cycle.

\begin{tabular}{|l|l|l|}
\hline I step & Denaturation & $98{ }^{\circ} \mathrm{C}(30-60 \mathrm{~s})$ \\
\hline II step & $\begin{array}{l}\text { Denaturation, annealing, extension } \\
(\mathrm{x} 30 \text { cycles })\end{array}$ & $98^{\circ} \mathrm{C}(10 \mathrm{~s}) \rightarrow 65^{\circ} \mathrm{C}^{*}(30 \mathrm{~s}) \rightarrow 72{ }^{\circ} \mathrm{C}(60 \mathrm{~s})$ \\
\hline III step & Final extension & $72{ }^{\circ} \mathrm{C}(10 \mathrm{~min}) \rightarrow 4^{\circ} \mathrm{C}$ \\
\hline
\end{tabular}

Table 11. PCR program for DNA amplification used for inverse PCR: denaturation (I step); denaturation, annealing, extension (II step) and final extension (III step). This program uses a touchdown PCR approach (*) where the temperature is decreasing from $67^{\circ} \mathrm{C}$ to $50^{\circ} \mathrm{C}$ by $0.5^{\circ} \mathrm{C}$ in the annealing step (step II) of every cycle.

\begin{tabular}{|l|l|l|}
\hline I step & Denaturation & $98^{\circ} \mathrm{C}(30 \mathrm{~s})$ \\
\hline II step & $\begin{array}{l}\text { Denaturation, annealing, } \\
\text { extension (x } 30 \text { cycles })\end{array}$ & $98{ }^{\circ} \mathrm{C}(10 \mathrm{~s}) \rightarrow 67{ }^{\circ} \mathrm{C}^{*}(10 \mathrm{~s}) \rightarrow 72{ }^{\circ} \mathrm{C}(70 \mathrm{~s})$ \\
\hline III step & Final extension & $72{ }^{\circ} \mathrm{C}(5 \mathrm{~min}) \rightarrow 4{ }^{\circ} \mathrm{C}$ \\
\hline
\end{tabular}




\subsubsection{Agarose gel electrophoresis; extraction of DNA fragments from agarose gel}

An agarose gel was made with $1 \%(\mathrm{w} / \mathrm{v})$ agarose dissolved in TAE buffer (40 mM Tris, $20 \mathrm{mM}$ acetic acid, $1 \mathrm{mM}$ EDTA, pH 8.0). The SERVA DNA stain clear G (Serva) was added in the gel for visualization of DNA $(30 \mu \mathrm{l} / \mathrm{l})$. Electrophoresis was performed with a constant voltage of $130 \mathrm{~V}$ for 30 minutes. DNA bands were visualized with Intas gel imager (Intas) and excised when needed. DNA extraction of the bands was carried out using Gel DNA Recovery Kit (Zymogen). Sample buffer (Thermo Fisher Scientific) was mixed with samples before they were loaded onto the gel. DNA fragments with adequate size were cut out from the agarose gel and purified with the DNA Clean \& Concentrator ${ }^{\mathrm{TM}_{-}} 5$ Kit according to manufacturer's instructions.

\subsubsection{Photometric determination of the concentration of DNA and DNA sequencing}

Concentrations of DNA samples were measured by UV-absorbance measurements at $260 \mathrm{~nm}$ using a Nanodrop spectrophotometer ND-1000 (Peqlab Biotechnologie). DNA samples were sequenced by GATC Biotech.

\subsubsection{Plasmid isolation}

For the purpose of sequencing and/or next steps of DNA amplification and cloning, DNA was purified from the culture media. A single colony was used and inoculated $5 \mathrm{ml} \mathrm{LB}$ medium. The medium was supplemented with adequate antibiotic. The culture was incubated at $37^{\circ} \mathrm{C}$, overnight, with constant shaking. The next day, culture was subjected to the plasmid extraction using QIAprep Spin Miniprep Kit (Qiagen). 


\subsubsection{FX cloning method}

The Fragment eXchange (FX) cloning method was performed according to the protocol from (Geertsma \& Dutzler, 2011). Primer design was done according to the recommendation of the protocol, so they contained 5'overhangs, for both forward (atatatGCTCTTCtAGT) and reverse (tatataGCTCTTCaTGC) primer. They also included recognition sites for the restriction enzyme LguI (SapI). Genes amplified from genomic DNA (3.2.1.5) were then used for a PCR reaction with FX cloning primers. PCR products were purified by agarose gel electrophoresis (3.2.1.6). For the first step of FX cloning, cloning into pINITcat was done, and vector and insert were mixed in a molar ratio of 1:5. Digestion of the product was done in Tango buffer with $2.5 \mathrm{U}$ LguI for 1 hour at $37{ }^{\circ} \mathrm{C}$. The enzyme was deactivated for 20 minutes at $65{ }^{\circ} \mathrm{C}$. $1.25 \mathrm{U}$ T4-Ligase and $10 \mathrm{mM}$ ATP were added and the reaction was incubated for 1 hour at $22{ }^{\circ} \mathrm{C}$. After inactivation $\left(20\right.$ minutes at $\left.65^{\circ} \mathrm{C}\right), 10 \mu \mathrm{l}$ of the sample was transformed into chemically competent E. coli MC1061 cells (3.2.1.3). Plasmids were isolated and sequenced $(3.2 .1 .8,3.2 .1 .7)$. Except the initial pINITcat vector, p7XC3H expression vector was used. After subcloning in expression vector (the same protocol as for the initial cloning), transformed cells were plated onto LB agar plate containing $50 \mu \mathrm{g} / \mathrm{ml}$ Kanamycin and additionally $7 \%$ sucrose. The ratio of pINITcat_gene (acrA_A200V,copA, cusA, cusA_D405N) to expression vector was 1:4.

\subsubsection{Inverse PCR}

Point mutation in cusA was introduced by inverse PCR (Ochman et al., 1988)). Back-to-back primers were designed with desired mutation ( $\mathrm{D}$ to $\mathrm{N}$ in residue 405). The blunt ends of the resulting linear PCR product (PCR program as in Table 11) are then phosphorylated and ligated in a two-step reaction upon purification by agarose gel extraction (3.2.1.6). In more detail, purified product was mixed with $10 \mathrm{mM}$ ATP and $1 \mathrm{U}$ Polynucleotide kinase (PNK), incubated for 30 minutes at $37{ }^{\circ} \mathrm{C}$ after which $5 \mathrm{mM}$ ATP and $1 \mathrm{U}$ T4-Ligase were added. Sample was incubated for 1 hour at $22{ }^{\circ} \mathrm{C}$ and transformed into E. coli chemically competent cells, strain MC1061 (3.2.1.3). Further, plasmid was isolated from a single colony and the sequence was checked $(3.2 .1 .8,3.2 .1 .7)$. 


\subsubsection{Biochemistry methods}

\subsubsection{Expression of acrA_A200V, acrB and tolC in E. coli}

Chemically competent $E$. coli C43(DE3) $\triangle a c r A B D$ were transformed with pET24a_acrB, pET24a_tolC and p7XC3H_acrA_A200V (3.2.1.3). After overnight growth, a single colony was inoculated in $100 \mathrm{ml}$ TB medium supplemented with $50 \mu \mathrm{g} / \mathrm{ml} \mathrm{KAN}$. The culture was grown overnight at $37{ }^{\circ} \mathrm{C}$ under constant shaking. The next morning, overnight cultures were diluted into fresh TB medium containing $50 \mu \mathrm{g} / \mathrm{ml}$ Kanamycin. The cells were cultured at $37{ }^{\circ} \mathrm{C}, 120 \mathrm{rpm}$ and grown to an $\mathrm{OD}_{600}$ of 0.5-0.8. Protein expression was induced with $0.5 \mathrm{mM}$ IPTG, and proceeded overnight at $20{ }^{\circ} \mathrm{C}$. All following steps were performed at 4 ${ }^{\circ} \mathrm{C}$. The cells were harvested by centrifugation at $7808 \mathrm{~g}$ in a FiberLite F9-6x1000 LEX rotor for 20 minutes. The cell pellet was collected and resuspended in $2 \mathrm{ml} / \mathrm{g} 20 \mathrm{mM}$ Tris- $\mathrm{HCl} \mathrm{pH}$ 7.5, $150 \mathrm{mM} \mathrm{NaCl}, 10 \mathrm{mM} \mathrm{MgCl}_{2}, 2 \mathrm{mM} \mathrm{CaCl} 2$ and $0.2 \mathrm{mM}$ DFP (or $1 \mathrm{mM}$ PMSF) supplemented with $10 \mu \mathrm{g} / \mathrm{ml}$ Lysozyme and $10 \mu \mathrm{g} / \mathrm{ml}$ DNase. The cell suspension was stirred for 1 hour and then lysed by two runs through Stansted SPCH-EP-10 pressure cell homogenizer (Homogenising Systems Ltd, UK) at $20 \mathrm{Kpsi}$. The lysate was cleared from cell debris by centrifugation for 45 minutes at $26074 \mathrm{~g}$ (rotor FiberLite F14-6x250y). To isolate the membranes, the supernatant was again centrifuged for 2 hours at $142000 \mathrm{~g}$ (Beckmann 45 Ti rotor). The membrane pellet was resuspended in $4 \mathrm{ml} / \mathrm{g} 20 \mathrm{mM}$ Tris- $\mathrm{HCl} \mathrm{pH}$ 7.5, $150 \mathrm{mM}$ $\mathrm{NaCl}$. The membrane aliquots were snap-frozen in liquid nitrogen and stored at $-80^{\circ} \mathrm{C}$.

\subsubsection{Purification of AcrA A200V, AcrB wild type, AcrB D407N and TolC}

The membranes containing acrA_A200V, acrB, acrB_D407N and tolC were thawed and supplemented with $1 \%$ n-Dodecyl- $\beta$-D-maltopyranoside (DDM). The membranes were solubilized for 1 hour while stirring at $4^{\circ} \mathrm{C}$. After centrifugation of the mixture for 35 minutes at $186000 \mathrm{~g}$ in a Beckmann $45 \mathrm{Ti}$ rotor, the supernatant was filtered $(0.22 \mu \mathrm{m})$ and applied to $1 \mathrm{ml} \mathrm{Ni-NTA}$ column equilibrated in $20 \mathrm{mM}$ Tris- $\mathrm{HCl} \mathrm{pH}$ 7.5, $150 \mathrm{mM} \mathrm{NaCl}, 0.02 \% \mathrm{DDM}$ 
and $10 \mathrm{mM}$ Imidazole. After batch binding for 1 hour, the flow through was collected and the nickel column was washed with $20 \mathrm{mM}$ Tris- $\mathrm{HCl} \mathrm{pH}$ 7.5, $150 \mathrm{mM} \mathrm{NaCl}$, supplemented with $0.02 \% \mathrm{DDM}$ and (1) $10 \mathrm{mM}$, (2) $40 \mathrm{mM}$, (3) $80 \mathrm{mM}$ and (4) $300 \mathrm{mM}$ Imidazole, for AcrB and AcrB_D407N or $0.03 \%$ DDM and (1) $10 \mathrm{mM}$, (2) $50 \mathrm{mM}$, (3) $200 \mathrm{mM}$ Imidazole, for AcrA_A200V and TolC. Washing steps 1 and 3 were performed with $10 \mathrm{CV}$ of buffer, step 2 with $15 \mathrm{CV}$ of buffer, for AcrB and AcrB_D407N or washing steps 1 and 2 with $20 \mathrm{CV}$, for AcrA_A200V and TolC. The proteins were eluted with 15 CV of the same buffer containing 300 mM Imidazole (AcrB and AcrB_D407N) or 200 mM (AcrA_A200V and TolC). Purified proteins were concentrated to $1 \mathrm{ml}$ using an Amicon Ultra-15 spin concentrator $50 \mathrm{kDa}$ cutoff (for AcrB, AcrB_D407N and TolC) and $30 \mathrm{kDa}$ cut-off (for AcrA_A200V), at 3,000 g in a Swing-out TTH 400 rotor (Heraeus) and applied to SEC using a Superose 6 10/300 increase column equilibrated with $20 \mathrm{mM}$ Tris- $\mathrm{HCl} \mathrm{pH}$ 7.5, $150 \mathrm{mM} \mathrm{NaCl}, 0.02 \%$ DDM (for AcrB, AcrB_D407N) or $0.03 \%$ DDM (for AcrA_A200V and TolC).

\subsubsection{Expression of $\operatorname{cop} A$ in $E$. coli}

Chemically competent E. coli C43(DE3) $\triangle a c r A B D$ were transformed with p7XC3H_copA (3.2.1.3). The next day, a single colony was picked from the LB agar plate and inoculated into $100 \mathrm{ml} \mathrm{LB}$ medium supplemented with $50 \mu \mathrm{g} / \mathrm{ml}$ Kanamycin. The culture was grown overnight at $37{ }^{\circ} \mathrm{C}$ under constant shaking. The next day, $91 \mathrm{LB}$ medium containing $50 \mu \mathrm{g} / \mathrm{ml}$ KAN were inoculated with the overnight culture. The cells were cultured at $37{ }^{\circ} \mathrm{C}, 120 \mathrm{rpm}$ and grown to an $\mathrm{OD}_{600}$ of $0.5-0.8$. Protein expression was induced with $1 \mathrm{mM}$ IPTG, and proceeded growing at $37{ }^{\circ} \mathrm{C}$ for 4 hours. The cells were suspended in $2 \mathrm{ml} / \mathrm{g}$ of buffer $\mathrm{S}: 20$ $\mathrm{mM}$ Tris $/ \mathrm{SO}_{4} \mathrm{pH} \quad 7.5,25 \mathrm{mM} \mathrm{Na}_{2} \mathrm{SO}_{4}, \quad 5 \quad \mathrm{mM}^{2} \quad \mathrm{MgSO}_{4}, 25 \quad \mathrm{mM} \quad \mathrm{K}_{2} \mathrm{SO}_{4}, \quad 1 \mathrm{mM} \beta-$ mercaptoethanol, $20 \%$ glycerol (v/v), and the buffer was supplemented with $1.2 \mathrm{mM}$ PMSF, $5 \mu \mathrm{g} / \mathrm{ml}$ DNaseI, $1 \mathrm{mM}$ EDTA and Lysosyme (1:1000). The cell suspension was stirred for 1 hour and then lysed by two runs through Stansted SPCH-EP-10 pressure cell homogenizer (Homogenising Systems Ltd, UK) at $20 \mathrm{Kpsi}$. Unbroken cell debris was removed with centrifugation for 45 minutes at $26074 \mathrm{~g}$ (rotor FiberLite F14-6x250y). To isolate the membranes, the supernatant was again centrifuged for 2 hours at $142000 \mathrm{~g}$ (Beckmann $45 \mathrm{Ti}$ rotor). The membrane pellet was resuspended in $4 \mathrm{ml} / \mathrm{g}$ of buffer $\mathrm{S}$. The membrane aliquots were snap-frozen in liquid nitrogen and stored at $-80{ }^{\circ} \mathrm{C}$. 


\subsubsection{Purification of CopA}

The thawed membrane suspension was complemented with $1 \mathrm{mM}$ PMSF and $2 \%$ DDM and subjected to the 1 -hour solubilization with stirring at $4{ }^{\circ} \mathrm{C}$. After centrifugation of the mixture for 40 minutes at $186000 \mathrm{~g}$ in a Beckmann 45 Ti rotor, the supernatant was filtered $(0.22 \mu \mathrm{m})$ and applied to $1 \mathrm{ml} \mathrm{Ni-NTA}$ column equilibrated in $20 \mathrm{mM}$ Tris- $\mathrm{Cl} \mathrm{pH} 7.5,5 \mathrm{mM} \mathrm{MgSO}_{4}$, $1 \mathrm{mM} \beta$-mercaptoethanol, $10 \%$ glycerol (v/v), 0,05\% DDM, $0.2 \mathrm{mM} \mathrm{PMSF,} 10 \mathrm{mM}$ Imidazole. After batch binding for 1 hour, the flow through was collected and the nickel column was washed with $20 \mathrm{mM}$ Tris-Cl $\mathrm{pH} 7.5,5 \mathrm{mM} \mathrm{MgSO}_{4}, 1 \mathrm{mM} \beta$-mercaptoethanol, $10 \%$ glycerol (v/v), $0.2 \mathrm{mM}$ PMSF, supplemented with $0.05 \%$ DDM and (1) $10 \mathrm{mM}$, (2) 50 $\mathrm{mM}$, (3) $80 \mathrm{mM}$ and (4) $250 \mathrm{mM}$ Imidazole. Washing steps 1 and 2 were performed with 15 $\mathrm{CV}$ of buffer, step 3 with $5 \mathrm{CV}$ of buffer. CopA was eluted with $20 \mathrm{CV}$ of the same buffer containing $250 \mathrm{mM}$ Imidazole. Purified CopA was concentrated to $1 \mathrm{ml}$ using an Amicon Ultra-15 spin concentrator $50 \mathrm{kDa}$ cut-off at $3000 \mathrm{~g}$ in a Swing-out TTH 400 rotor (Heraeus) and applied to SEC using a Superose 6 10/300 increase column equilibrated with $20 \mathrm{mM}$ HEPES/OH pH 7.5, 5 mM MgSO 4 , 1mM $\beta$-mercaptoethanol, $10 \%$ glycerol, $0.2 \%$ PMSF, $0,05 \%$ DDM.

\subsubsection{Expression of cusA and cusA_D405N in E. coli}

Chemically competent $E$. coli C43(DE3) $\triangle a c r A B D$ were transformed with p7XC3H_cusA and p7XC3H_cusA_D405N (3.2.1.3). The next day, a single colony was picked from the LB agar plate and inoculated into $100 \mathrm{ml} \mathrm{LB}$ medium supplemented with $50 \mu \mathrm{g} / \mathrm{ml} \mathrm{Kanamycin}$. The culture was grown overnight at $37{ }^{\circ} \mathrm{C}$ under constant shaking. The next day, LB medium containing $50 \mu \mathrm{g} / \mathrm{ml} \mathrm{KAN}$ was inoculated with the overnight culture. The cells were cultured at $37{ }^{\circ} \mathrm{C}, 120 \mathrm{rpm}$ and grown to an $\mathrm{OD}_{600}$ of 0.5-0.8. Protein expression was induced with $1 \mathrm{mM}$ IPTG, and proceeded growing at $25{ }^{\circ} \mathrm{C}$ overnight. The cells were suspended in $2 \mathrm{ml} / \mathrm{g}$ of buffer F: $100 \mathrm{mM}$ Disodium phosphate $\mathrm{pH}$ 7.2, $1 \mathrm{mM}$ EDTA, $10 \%$ glycerol (v/v) and $1 \mathrm{mM}$ PMSF, and the buffer was supplemented with $1 \mathrm{mM}$ PMSF, $5 \mu \mathrm{g} / \mathrm{ml}$ DNaseI, $1 \mathrm{mM}$ EDTA and Lysosyme (1:1000). The cell suspension was stirred for 1 hour and then lysed by two runs through Stansted SPCH-EP-10 pressure cell homogenizer (Homogenising 
Systems Ltd, UK) at $20 \mathrm{Kpsi}$. Unbroken cell debris was removed with centrifugation at for 45 minutes at $26074 \mathrm{~g}$ (rotor FiberLite F14-6x250y). To isolate the membranes, the supernatant was again centrifuged for 2 hours at $142000 \mathrm{~g}$ (Beckmann 45 Ti rotor). The membrane pellet was resuspended in $4 \mathrm{ml} / \mathrm{g}$ of buffer $\mathrm{F}$. The membrane aliquots were snap-frozen in liquid nitrogen and stored at $-80^{\circ} \mathrm{C}$.

\subsubsection{Purification of CusA and CusA D405N}

The thawed membrane suspension was complemented with $1 \mathrm{mM}$ PMSF and $2 \%$ DDM and subjected to the 1-hour solubilization with stirring at $4^{\circ} \mathrm{C}$. After centrifugation of the mixture for 30 minutes at $186000 \mathrm{~g}$ in a Beckmann 45 Ti rotor, the supernatant was filtered $(0.22 \mu \mathrm{m})$ and applied to $1 \mathrm{ml}$ Ni-NTA column equilibrated in $20 \mathrm{mM}$ HEPES-NaOH $\mathrm{pH}$ 7.6, $1 \mathrm{mM}$ PMSF, $10 \%$ glycerol (v/v), 0.05\% DDM, $10 \mathrm{mM}$ Imidazole. After batch binding for 1 hour, the nickel column was washed with $20 \mathrm{mM}$ HEPES-NaOH pH 7.6, $1 \mathrm{mM}$ PMSF, $10 \%$ glycerol (v/v), $10 \mathrm{mM}$ Imidazole, supplemented with $0.05 \%$ DDM and (1) $10 \mathrm{mM},(2)$ $50 \mathrm{mM}$, (3) $100 \mathrm{mM}$ and (4) $250 \mathrm{mM}$ Imidazole. Washing step 1 was performed with $15 \mathrm{CV}$ of buffer, step 2 with $10 \mathrm{CV}$, washing step 3 with $5 \mathrm{CV}$ of buffer. CusA wild type and CusA_D405N were eluted with $20 \mathrm{CV}$ of the same buffer containing $250 \mathrm{mM}$ Imidazole. Purified CusA and CusA_D405N was concentrated to $1 \mathrm{ml}$ using an Amicon Ultra-15 spin concentrator $50 \mathrm{kDa}$ cut-off at $3000 \mathrm{~g}$ in a Swing-out TTH 400 rotor (Heraeus) and applied to SEC using a Superose 6 10/300 increase column equilibrated with $20 \mathrm{mM}$ HEPES-NaOH pH 7.6, 0.05\% DDM, $200 \mathrm{mM} \mathrm{KCl.}$

\subsubsection{Blue Native-PAGE}

For analysis of the oligomeric state of produced proteins, Blue Native PAGE (BN-PAGE) was used. Protein sample in detergent was mixed with BN-sample buffer $(2$ x concentrated: $0.5 \%$ Coomassie-G250, $50 \mathrm{mM}$ E-aminocaproic acid, $10 \mathrm{mM}$ Bis-Tris/HCl pH 7.0). Samples were applied on 4-20\% Mini-PROTEAN TGX Precast Protein Gels (Bio-Rad). Two different buffers were used for cathode: buffer $1(15 \mathrm{mM}$ Bis-Tris/ $\mathrm{HCl} \mathrm{pH} 7.0,0.02 \%$ 
Coomassie G250), and buffer 2 (15 mM Bis-Tris-HCl pH 7.0, $0.002 \%$ Coomassie-G250). The Anode buffer is $50 \mathrm{mM}$ Bis-Tris- $\mathrm{HCl} \mathrm{pH} 7.0,50 \mathrm{mM}$ Tricine. After 15 minutes at constant voltage of $100 \mathrm{~V}$ and 1 hour at $300 \mathrm{~V}$, Cathode buffer 1 was exchanged to Cathode buffer 2. $300 \mathrm{~V}$ were applied for additional 2 hours afterwards. The gel was distained overnight in $30 \%(\mathrm{v} / \mathrm{v}) \mathrm{MeOH}, 10 \%(\mathrm{v} / \mathrm{v})$ acetic acid.

\subsubsection{SDS-polyacrylamide gel electrophoresis (SDS-PAGE)}

Protein samples were analysed with SDS-PAGE, which allows separation by molecular weight (LAEMMLI, 1970). Composition of the gels used is shown in Table 12.

Table 12. Composition of the SDS-page gels.

\begin{tabular}{|l|l|l|}
\hline & Separating gel & Stacking gel \\
\hline Acrylamide concentration & $10 \%$ & $4 \%$ \\
\hline H20 & $4 \mathrm{ml}$ & $2.7 \mathrm{ml}$ \\
\hline $\mathbf{3 0} \%$ acrylamide mix (29:1 acrylamide: bisacrylamide) & $3.3 \mathrm{ml}$ & $0.67 \mathrm{ml}$ \\
\hline $\mathbf{1 . 5}$ M Tris-Cl pH 8.8 & $2.5 \mathrm{ml}$ & $/$ \\
\hline $\mathbf{1}$ M Tris-Cl pH 6.8 & $/$ & $0.5 \mathrm{ml}$ \\
\hline $\mathbf{1 0} \%$ SDS & $0.1 \mathrm{ml}$ & $0.04 \mathrm{ml}$ \\
\hline $\mathbf{1 0} \%$ APS & $0.1 \mathrm{ml}$ & $0.04 \mathrm{ml}$ \\
\hline TEMED & $0.004 \mathrm{ml}$ & $0.004 \mathrm{ml}$ \\
\hline
\end{tabular}

Protein samples were mixed with sample buffer (45 mM Tris-Cl, $\mathrm{pH} \mathrm{6.8,} 10 \%$ (v/v) glycerol, $1 \%(\mathrm{w} / \mathrm{v})$ SDS, $50 \mathrm{mM} \%(\mathrm{v} / \mathrm{v})$ B- mercaptoethanol, $0.01 \%$ (w/v) bromophenol blue), incubated at $37{ }^{\circ} \mathrm{C}$ for 5 minutes and centrifuged for 10 minutes at $21000 \mathrm{~g}$. Separation of proteins was carried out by 15 minutes at $100 \mathrm{mV}$ (stacking gels), followed by 1 hour at $160 \mathrm{mV}$ (separating gel), in running buffer (25 mM Tris, $250 \mathrm{mM}$ glycine, $0.1 \%$ (w/v) SDS). 


\subsubsection{Preparation of the samples for SDS-PAGE: whole cells, solubilized cells, protein purification}

Protein purification samples: All fractions collected (from membrane solution until the purified protein) during Immobilized Metal Affinity Chromatography (IMAC) as well as from Size Exclusion Chromatography (SEC) purification were collected and mixed with SDS-PAGE sample buffer (5x Protein sample buffer: $45 \mathrm{mM}$ Tris-Cl, $\mathrm{pH}$ 6.8, $10 \%$ (v/v) glycerol, $1 \%(\mathrm{w} / \mathrm{v})$ SDS, $50 \mathrm{mM} \%(\mathrm{v} / \mathrm{v})$ B- mercaptoethanol, $0.01 \%(\mathrm{w} / \mathrm{v})$ bromophenol blue).

Solubilized cells for expression test: Whole cell pellets obtained from a $1 \mathrm{ml}$ culture at $\mathrm{OD}_{600}$ of 10 were resuspended in $2000 \mu \mathrm{l}$ of the corresponding Lysis buffer $(20 \mathrm{mM}$ Tris/Cl $\mathrm{pH} 8$, $500 \mathrm{mM} \mathrm{NaCl}, 2 \mathrm{mM} \mathrm{MgCl} 2$ ) supplemented with $10 \mu \mathrm{g} / \mathrm{ml}$ Lysozyme, $10 \mu \mathrm{g} / \mathrm{ml}$ DNase and $200 \mu \mathrm{M}$ phenylmethyl sulfonyl fluoride (PMSF). Cells were lysed by shearing force with a Fastprep machine (using glass beads) and samples were centrifuged for 20 minutes at 20000 g. Supernatants were suspended to ultracentrifugation for 10 minutes at $240000 \mathrm{~g}$ in a F50L$24 \times 1.5$ rotor. Pellet was suspended into $100 \mathrm{ul}$ buffer containing $1 \% \mathrm{DDM}$, and mixed for 1 hour at $4{ }^{\circ} \mathrm{C}$. The samples were then again ultracentrifuged for 20 minutes at $240000 \mathrm{~g}$. Samples before and after ultracentrifugation were mixed with 5x Protein sample buffer (see above for final concentrations) and samples were applied to SDS-PAGE.

Proteoliposome sample: for applying to the SDS-PAGE gel, samples containing proteoliposomes were mixed with SDS-PAGE sample buffer and loaded to the SDSpolyacrylamide gel.

\subsubsection{Coomassie staining}

For Coomassie staining, SDS-polyacrylamide gels were treated with a fixation solution (50\% EtOH, $10 \%$ Acetate) for 10 minutes at room temperature. Gels were incubated with a Staining solution (41.5\% (v/v) EtOH, $5.25 \%$ (v/v) acetic acid, $0.03 \%$ Coomassie-G250) and heated up (20 seconds in the microwave, at $500 \mathrm{~V}$ ). The heated gel in Staining solution was incubated for 1 hour at room temperature, followed by the exchange of the Staining solution 
with the Destaining solution (30\% (v/v) MeOH, $10 \%(\mathrm{v} / \mathrm{v})$ acetic acid) and kept at room temperature until the gel matrix was cleared of the dye, revealing protein as blue bands.

\subsubsection{Silver staining}

Another method was used for detection of the proteins on the SDS-polyacrylamide gel, silver staining. Method was performed according to Nesterenko et al (Nesterenko et al., 1994). SDS-polyacrylamide gel was placed into the Fixation solution ((50\% (v/v) acetone, $1.25 \%$ (w/v) TCA, $0.015 \%(\mathrm{v} / \mathrm{v})$ formaldehyde) for 5 minutes, after which it was washed with water. In the next step, the gel was incubated with $50 \%(\mathrm{v} / \mathrm{v})$ acetone for 5 minutes, and subsequently with $0.03 \%(\mathrm{w} / \mathrm{v}) \mathrm{Na}_{2} \mathrm{~S}_{2} \mathrm{O}_{3}$ for 1 minute, following another washing with water. The gel was kept in the Staining solution $\left(0.25 \%(\mathrm{w} / \mathrm{v}) \mathrm{AgNO}_{3}, 0.37 \%(\mathrm{v} / \mathrm{v})\right.$ formaldehyde) for 8 minutes, and then washed with water. For visualization, the Developing solution was used ( $\left(2 \%\right.$ (w/v) $\mathrm{Na}_{2} \mathrm{CO}_{3}, 0.37 \%$ (v/v) formaldehyde, $0.0075 \%$ (w/v) $\mathrm{Na}_{2} \mathrm{~S}_{2} \mathrm{O}_{3}$ ). After detection of the first bands, the reaction was stopped with $1 \%$ acetic acid.

\subsubsection{In-gel fluorescence}

Proteins that have a fluorescent tag were detected on the SDS-polyacrylamide gel via In-gel fluorescence. Membrane fusion protein (MFP), EmrA-sfGFP-cMyc, was detected at $\lambda_{\mathrm{ex}} / \lambda_{\mathrm{em}}-$ $460 \mathrm{~nm} / 515 \mathrm{~nm}$, while RND component, EmrB-mRFP1-His10, was detected at $\lambda_{\mathrm{ex}} / \lambda_{\mathrm{em}}-$ $630 \mathrm{~nm} / 670 \mathrm{~nm}$. Gels were analysed using ImageQuant LAS4000.

\subsubsection{Immunoblotting}

Immunoblotting was performed using semidry blotting technique, according to Towbin et al (Towbin et al., 1979). Transfer buffer (48 mM Tris, $39 \mathrm{mM}$ glycine, $20 \%$ (v/v) methanol and $0.01 \%(\mathrm{w} / \mathrm{v}) \mathrm{SDS}, \mathrm{pH}$ 9.2) was used for soaking filter paper before assembling the sandwich 
"filter paper- the nitrocellulose membrane-SDS-polyacrylamide gel-filter paper". The transfer was done in the Bio-Rad Turbo blotting machine with the Standard SD mode protocol, 1.0 A, $25 \mathrm{~V}$ for 30 minutes. The nitrocellulose membrane was then incubated in the Blocking solution (TBST - $10 \mathrm{mM}$ Tris-HCl pH 8.0, $150 \mathrm{mM} \mathrm{NaCl}, 0.05 \%$ (v/v) Tween 20 with $3 \%$ (w/v) BSA) for at least 1 hour at room temperature and with mild agitation. After incubation, the membrane was washed three times for 5 minutes in TBST solution. The membrane was incubated for 1 hour with primary antibody solution (TBST with $1 \%$ BSA supplemented with adequate antibody to buffer ratio). When necessary, the second antibody was used, following membrane washing 3 times in TBST solution between two antibodies. After the antibody incubation, the membrane was washed 3 times for 5 minutes with TBST buffer (10 $\mathrm{mM}$ Tris-Cl, $\mathrm{pH} 8.0,150 \mathrm{mM} \mathrm{NaCl}, 0.05 \%(\mathrm{v} / \mathrm{v})$ Tween 20) and 3 times for 5 minutes with TMN solution (100 mM Tris-Cl, pH 9.5, $100 \mathrm{mM} \mathrm{NaCl}$ and $5 \mathrm{mM} \mathrm{MgCl}_{2}$ ). Finally, the membrane was soaked in Developing solution $(0.33 \mathrm{mg} / \mathrm{mL}$ NBT, $0.225 \mathrm{mg} / \mathrm{mL}$ BCIP dissolved in TMN buffer) until first bands were detected. Reaction was stopped by washing the membrane extensively with water.

Table 13. Antibody description.

\begin{tabular}{|l|l|l|}
\hline Primary antibody & Company & Dilution \\
\hline $\begin{array}{l}\text { Monoclonal Anti-polyHistidine- } \\
\text { Alkaline Phosphatase }\end{array}$ & Sigma Aldrich & $1: 4000$ \\
\hline $\begin{array}{l}\text { Precision Protein } \\
\text { Conjugate }\end{array}$ & & $1: 2000$ \\
\hline Antri-Myc Tag Antibody, clone 4A6 & Sigma Aldrich & \\
\hline Secondary antibody & Bio-Rad & $1: 2000$ \\
\hline Anti-mouse & Sigma Aldrich & Dilution \\
\hline
\end{tabular}

\subsubsection{Determination of the protein concentration}

Determination of protein concentration was done using Nanodrop spectrophotometer ND1000 (Peqlab Biotechnologie). Photometric determination was performed by measuring the absorbance at $280 \mathrm{~nm}$ (Wilkins et al., 1999). Molar absorption coefficients were calculated for each protein utilizing online tool Protparam (https://web.expasy.org/protparam/). 


\subsubsection{Liposome preparation}

Round bottom flask was cleaned with ethanol. Residual ethanol is acceptable. With the glass pipette, lipid solution in Chloroform was pipetted into the flask. For AcrB, CopA, CusA proteoliposome assays (3.2.3.1, 3.2.3.4, 3.2.3.5), Cholesterol and DOPC in a ratio 1:3 (w/w). For the pull-down assay, additionally DOPE-biotin cap (18:1) for the final ratio 1:10 (w/w) was added. For TolC assays (3.2.2.20, 3.2.3.2), E. coli polar lipids and DOPC were used in ratio $7: 3(\mathrm{w} / \mathrm{w})$. Lipid solution was dried at a rotary evaporator at 474 mbar for 45-50 minutes, at $30{ }^{\circ} \mathrm{C}\left(50{ }^{\circ} \mathrm{C}\right.$ when Cholesterol was used). Drying was then continued at maximum vacuum pressure for 1 hour. Lipid film was then hydrated with corresponding buffer till the desired final concentration $(3.2 .2 .17,3.2 .2 .18)$. The lipids were afterwards heated in the water bath at $37^{\circ} \mathrm{C}$ for 10 minutes. Solution was then sonicated (Branson Sonifier 250) for 10 minutes (30 second pulse, 30 second break). Aliquoted $1 \mathrm{ml}$ liposomes were subjected to freeze and thaw cycles $\left(5\right.$ cycles, thaw at $\left.55^{\circ} \mathrm{C}\right)$. At the final freezing step in liquid nitrogen, they were stored at $-80{ }^{\circ} \mathrm{C}$, until usage. Stored like this, liposomes are stable at least 6 months.

\subsubsection{Determination of $\mathbf{R}_{\text {sat }} / \mathbf{R}_{\text {sol }}$}

Thawed liposomes were extruded through a polycarbonate filter membrane (LiposoFast Liposome Factory polycarbonate membrane, Sigma Aldrich, Darmstadt, Germany) in order to obtain liposomes of equal size. The filter size depends on the protein to be reconstituted (3.2.2.17). Filtered liposomes were then pipetted into a 96 well plate, $100 \mu \mathrm{l}$ in each well. In each well an increasing detergent amount was added. For AcrB related liposomes, $\beta$-OG was added in a range of $0.3-0.7 \%$ (with an increment of $0.1 \%$ ). The detergent was pipetted carefully into the liposome solution. The exact liposome preparation depends on the protein they are intended for (3.2.2.15). Solubilization of the liposomes then took place for one hour at room temperature with constant rocking. Afterwards, absorption at $540 \mathrm{~nm}$ was measured using Tecan Microplate Reader (Tecan, Switzerland). 


\subsubsection{Reconstitution of AcrA A200V, AcrB, AcrB D407N, AcrB F628L and TolC in LUVs}

Prepared liposomes (3.2.2.15; lipid concentration- $1 \mathrm{mg} / \mathrm{ml}$ ) were extruded 11 times through a $200 \mathrm{~nm}$ polycarbonate filter membrane. Liposomes intended for AcrB (as well as for AcrAB, AcrB D407N, AcrAB D407N, AcrB F628L) reconstitution were additionally extruded through $100 \mathrm{~nm}$ pore-size filter. Liposomes for TolC reconstitution were extruded through $200 \mathrm{~nm}$ polycarbonate filter membrane. At the indicated concentrations, detergent was added to liposomes so that the solution contains solubilized liposomes and mixed micelles. For DOPC/Cholesterol liposomes (AcrA/AcrB proteoliposomes) and DOPC/E. coli polar liposomes (TolC proteoliposomes), $0.55 \% \beta$-OG was added for $1 \mathrm{mg}$ of lipids. Solubilization took place for 1 hour at $4{ }^{\circ} \mathrm{C}$. Solubilized liposomes were mixed with proteins in ratios: for AcrB - 1:20 (protein to lipid ratio; (w/w)); for TolC - 1:10 (protein to lipid ratio; (w/w)); AcrA was added 2.5:1 (AcrA to AcrB ratio; (w/w)) (AcrB concentration: $50 \mu \mathrm{g} / \mathrm{ml}$, AcrA concentration: $125 \mu \mathrm{g} / \mathrm{ml}$, TolC concentration: $100 \mu \mathrm{g} / \mathrm{ml})$. Together with proteins, pyranine ( $2 \mathrm{mM}$, final concentration) was added to the mixture. The proteins-lipids mixture was incubated on a spin wheel at $4{ }^{\circ} \mathrm{C}$ for 45 minutes. For the purpose of detergent removal, Bio-Beads were added, $30 \mathrm{mg}$ of beads per $1 \mathrm{mg}$ of detergent. The total amount of Bio-Bead was added in four portions (7.5 $\mathrm{mg}$ of Bio-Beads per $1 \mathrm{mg}$ detergent per portion): two portions one hour apart for overnight washing, and two the next day one hour apart. All the steps were done with the samples placed on a spin wheel. The supernatant was collected (Bio-Beads removal with careful pipetting) and then the proteoliposomes were passed through a NAP-10 column, in order to remove the external pyranine. The proteoliposomes were eluted from the NAP-10 column with reconstitution buffer. For additional washing and dye removal, proteoliposomes were centrifuged at $279000 \mathrm{~g}$ in TLA 120 rotor Beckman Coulter TLX-120, for 30 minutes, rinsed with reconstitution buffer (10 mM HEPES/KOH pH 7.6, $5 \mathrm{mM} \mathrm{Mg}_{2} \mathrm{SO}_{4}, 150 \mathrm{mM} \mathrm{K}_{2} \mathrm{SO}_{4}$ ), and again centrifuged for $30 \mathrm{~min}$ as described above.

For the TolC pyranine wash assay, proteoliposomes and control liposomes were prepared in reconstitution buffer (20 mM Tris/Cl, $150 \mathrm{mM} \mathrm{NaCl} \mathrm{pH} \mathrm{7.5),} \mathrm{with} \mathrm{DOPC} \mathrm{and} \mathrm{E.} \mathrm{coli} \mathrm{polar}$ lipids mixture 3:7 ratio (v/v). Liposomes were extruded 17 times through a $400 \mathrm{~nm}$ polycarbonate filter membrane and then destabilized by addition $4 \%$ Triton X-100 (v/v), final concentration $0.25 \%$. Lipids and detergent were incubated for 30 minutes at $4{ }^{\circ} \mathrm{C}$. All 
following steps were done at $4{ }^{\circ} \mathrm{C}$. Purified protein was added at 1:20 (w/w) and incubated for 1 hour. Detergent removal was performed with addition of Bio-Beads (16 mg of BioBeads per $1 \mathrm{mg}$ of detergent) that took place overnight, and total amount of beads added was divided into 4 portions ( $4 \mathrm{mg}$ of Bio-Beads per $1 \mathrm{mg}$ detergent per portion) subsequently added to the sample (two the same day, two next day, one hour apart each).

\subsubsection{Reconstitution of CopA, CusA wild type and CusA D405N in LUVs}

Prepared liposome solution (3.2.2.15; lipid concentration- $5 \mathrm{mg} / \mathrm{ml}$ ) was subjected to the extrusion through the membrane with a pore size of $400 \mathrm{~nm}$ in diameter. The extrusion was performed 11 times through the polycarbonate filter membrane. Afterwards, liposomes were mixed with purified CopA, CusA wild type or CusA D405N (protein to lipid ratio: 1:50 (w/w) for CopA, CopA concentration: $100 \mu \mathrm{g} / \mathrm{ml}$ and 1:45 (w/w) for CusA, CusA concentration: $111.11 \mu \mathrm{g} / \mathrm{ml}$ ), or with the respective purification buffer (for the empty liposomes control) for 1 hour at $4{ }^{\circ} \mathrm{C}$. The samples were placed on a rotating spin wheel. The solution was then subjected to four freeze and thaw cycles. Then, proteoliposomes were extruded again, 5 times, through a $400 \mathrm{~nm}$ pore-sized filter. In the case of CopA reconstitution, the dye PGSK $(100 \mu \mathrm{M})$ was added together with the protein to the liposomes before the start of the freeze and thaw cycles. After the freeze and thaw cycles, the solution with the PGSK dye was applied to the NAP-10 column for the removal of external dye, preequilibrated with the reconstitution buffer. The proteoliposomes were eluted with the reconstitution buffer and subjected to an additional extrusion through a $400 \mathrm{~nm}$ pore-sized filter for 5 times. The reconstitution buffer for CopA reconstitution - $20 \mathrm{mM} \mathrm{HEPES/KOH}$ $\mathrm{pH}$ 6.6, $50 \mathrm{mM} \mathrm{K}_{2} \mathrm{SO}_{4}$; for CusA reconstitution - $20 \mathrm{mM} \mathrm{HEPES} / \mathrm{KOH} \mathrm{pH} \mathrm{7.6,50} \mathrm{mM}$ $\mathrm{K}_{2} \mathrm{SO}_{4}$ or $20 \mathrm{mM}$ HEPES/KOH pH 6.6, $50 \mathrm{mM} \mathrm{K}_{2} \mathrm{SO}_{4}$. 


\subsubsection{Solubilization assay}

Proteoliposomes (3.2.2.17, 3.2.2.18), $200 \mu \mathrm{l}$, were centrifuged at $279000 \mathrm{~g}, 4{ }^{\circ} \mathrm{C}$ in TLA 100 rotor, Beckman Coulter TLX-120, for 30 minutes. Obtained supernatant was discarded and the pellet resuspended in $125 \mu \mathrm{l}$ of reconstitution buffer $(3.2 .2 .17,3.2 .2 .18)$. DDM (2 \%, final concentration) was added to the proteoliposomes solution for the solubilization step that takes place at $4{ }^{\circ} \mathrm{C}$, with constant rotation on a spin wheel, for 1 hour. Afterwards, $25 \mu \mathrm{l}$ sample was mixed with SDS-PAGE sample buffer (sample before ultracentrifugation). The remaining $100 \mu \mathrm{l}$ was centrifuged at $213000 \mathrm{~g}, 4{ }^{\circ} \mathrm{C}$ in TLA 100 rotor, Beckman Coulter TLX-120, for 10 minutes. $80 \%$ of the supernatant was taken and mixed with SDS-PAGE sample buffer (sample after centrifugation).

\subsubsection{Pyranine fluorescence and $\mathrm{pH}$ dependence; PhenGreen SK titration with silver ions; TolC pyranine wash assay}

Pyranine $\mathrm{pH}$ dependence was tested in buffer - $20 \mathrm{mM}$ HEPES (500 uM pyranine). The $\mathrm{pH}$ of the buffer was adjusted as indicated on Figure 24, from $\mathrm{pH} 5.2$ until $\mathrm{pH}$ 8.2. Measurements were done in Fluorescence Spectrometer Cary Eclipse Varian, excitation - 460 nm, emission $-510 \mathrm{~nm}$.

Phen Green SK was tested in buffer $2 \mathrm{mM}$ HEPES pH 7.5, $100 \mathrm{mM} \mathrm{K}_{2} \mathrm{SO}_{4}$. When indicated $5 \mathrm{mM} \mathrm{MgCl} 2$ was present in the buffer with or without $0.1 \mathrm{mM}$ EDTA (Figure 53). PGSK dye was added in concentrations $200 \mu \mathrm{M}, 1 \mu \mathrm{M}$ or $200 \mathrm{nM}$ and silver nitrate as indicated in Figure 52. All the measurements were performed using Tecan Microplate Reader (Tecan, Switzerland) at excitation $-480 \mathrm{~nm}$, emission $-530 \mathrm{~nm}$.

For the TolC pyranine wash assay, proteoliposomes and control liposomes were diluted in reconstruction buffer $(20 \mathrm{mM}$ Tris/Cl, $150 \mathrm{mM} \mathrm{NaCl}, \mathrm{pH} 7.5)$ in ratio 1:10, v/v. The samples were incubated on ice for at least 15 minutes. Afterwards, samples were applied onto the filter membrane $(0.22 \mu \mathrm{m}$ GSFT Merck Millipore) previously washed with reconstitution buffer. After filtration of the sample, filter was washed with the same buffer. Filter was then incubated for 10 minutes in PBS buffer supplemented with $1 \%$ SDS, with constant shaking 
at room temperature. The sample was transferred to the 96 well plate and the fluorescence of pyranine was measured (excitation - $460 \mathrm{~nm}$, emission - $510 \mathrm{~nm}$, using Tecan Microplate Reader (Tecan, Switzerland)). 


\subsubsection{Functional assays}

\subsubsection{Functional assay of AcrB in LUVs}

AcrB reconstituted into liposomes (AcrB wild type or AcrB variants, with or without AcrA) were used to monitor substrate-dependent proton uptake. Fluorescence measurements were conducted at $25{ }^{\circ} \mathrm{C}$ with continuous stirring using Horiba Jobin Yvon Fluorolog 3. Pyranine dye $(2 \mathrm{mM})$ was incorporated inside the liposomes during reconstitution. $100 \mu \mathrm{l}$ of proteoliposomes (protein to lipid ratio: 1:20 (w/w); AcrB concentration: $50 \mu \mathrm{g} / \mathrm{ml}$, AcrA concentration: $125 \mu \mathrm{g} / \mathrm{ml}$ ) or control liposomes were diluted in $900 \mu \mathrm{l}$ of assay buffer for the start of the measurement. The buffer added for the assay was acidic buffer, with the $\Delta \mathrm{pH}$ of 1 (10 mM HEPES/KOH pH 6.6, $5 \mathrm{mM} \mathrm{Mg}_{2} \mathrm{SO}_{4}, 150 \mathrm{mM} \mathrm{K}_{2} \mathrm{SO}_{4}$ ) in comparison to the buffer inside the liposomes (10 mM HEPES/KOH pH 7.6, $5 \mathrm{mM} \mathrm{Mg}_{2} \mathrm{SO}_{4}, 150 \mathrm{mM} \mathrm{K} \mathrm{SO}_{4}$ ). When indicated, $1 \mathrm{mM}$ final concentration sodium taurocholate (taurocholate is a substrate of AcrB), and/or inhibitors MBX3132 or PA $\beta \mathrm{N}$ (each at $1 \mu \mathrm{M}$, final concentration) were added. Slits for excitation and emission -2 and $5 \mathrm{~nm}$, respectively; excitation wavelength $-460 \mathrm{~nm}$, emission wavelength $-510 \mathrm{~nm}$.

\subsubsection{Complex formation of AcrAB-TolC complex in vitro}

$100 \mu \mathrm{l}$ of AcrAB (or AcrB) proteoliposomes (lipid concentration: $1 \mathrm{mg} / \mathrm{ml}$; AcrB concentration: $50 \mu \mathrm{g} / \mathrm{ml}$, AcrA concentration: $125 \mu \mathrm{g} / \mathrm{ml}$ ) were mixed with $100 \mu \mathrm{l}$ TolC proteoliposomes (lipid concentration: $1 \mathrm{mg} / \mathrm{ml}$; TolC concentration: $100 \mu \mathrm{g} / \mathrm{ml}$ ). Proteoliposomes contained $10 \mathrm{mM}$ HEPES pH 7.6/KOH, $150 \mathrm{mM} \mathrm{K}_{2} \mathrm{SO}_{4}, 5 \mathrm{mM} \mathrm{MgSO}$. $790 \mu \mathrm{l}$ of reconstitution buffer was added then to the proteoliposomes, together with $1 \mu \mathrm{M}$ MBX3132, or $1 \mathrm{mM}$ taurocholate. Upon addition of buffer (10 mM HEPES pH 7.6/KOH, $150 \mathrm{mM} \mathrm{K} \mathrm{SO}_{4}, 5 \mathrm{mM} \mathrm{MgSO}_{4}$ ), the mixture was incubated for 10 minutes at room temperature, with slight agitation. Then, $10 \mu \mathrm{l}$ of Streptavidin coated magnetic beads (MagStrep "type3" XT Beads, 5 \% (v/v) suspension, Iba, Göttingen, Germany, if not stated that some other beads were used instead) were added to the mix, and the sample was placed 
on a magnetic separator. With this, TolC-biotinylated proteoliposomes (or AcrABbiotinylated proteoliposomes) were magnetized and pelleted together with their interaction partners (AcrAB proteoliposomes or TolC proteoliposomes in the case of AcrAB-biotinylated proteoliposomes). Within minutes, a pellet was formed. The pellet was washed twice with 1 $\mathrm{ml}$ of buffer (100 mM Tris/Cl pH 7.5, $150 \mathrm{mM} \mathrm{NaCl}$ ). Finally, the pellet was suspended in 5x SDS loading buffer and directly loaded onto a $10 \%$ SDS-page gel. The bands of the protein were revealed with Silver staining.

In order to examine the influence of the incubation time: AcrAB proteoliposomes and TolC proteoliposomes $(100 \mu \mathrm{l}$ each) were mixed for $60 \mathrm{~min}$, without substrate or inhibitor, followed by addition of $10 \mu \mathrm{l}$ Streptavidin coated magnetic beads. As described above, the pellet was washed and suspended in 5x SDS loading buffer and directly loaded onto a $10 \%$ SDS-page gel.

In order to examine the influence of $\mathrm{pH}$ gradient: $100 \mu \mathrm{l}$ AcrAB proteoliposomes and $100 \mu \mathrm{l}$ TolC proteoliposomes were mixed with constant rotation on a spin wheel, for 10 minutes. To the sample, $900 \mu \mathrm{l}$ acidic buffer (10 mM HEPES pH 6.6/KOH, $150 \mathrm{mM} \mathrm{K} \mathrm{SO}_{4}, 5 \mathrm{mM}$ $\mathrm{MgSO}_{4}$ ) was added, containing $1 \mathrm{mM}$ sodium taurocholate. At $\mathrm{t}=0,10 \mu \mathrm{l}$ magnetic beads were added and a pellet was formed as described above. The pellet was washed twice. The same procedure was done at $\mathrm{t}=5$ (five minutes after addition of acidic buffer with the substrate). Samples were collected for SDS-PAGE analysis.

\subsubsection{Determination of the Michaelis-Menten constant $\left(K_{m}\right)$ for ATP of} CopA

ATP hydrolysis activity was measured by a NADH-coupled ATP regenerating system at $25^{\circ} \mathrm{C}$ according to the adapted protocol from Warren et al, from 1974 (Warren et al., 1974). ATPase activity was measured in a reaction that uses the coupled pyruvate kinase (PK)/lactate dehydrogenase (LDH) assay to report on the ATP hydrolysis by monitoring NADH oxidation as decrease in absorbance at $340 \mathrm{~nm}$. Reaction buffer contained $2 \mathrm{mM}$ HEPES pH 7.5, $0.1 \mathrm{M} \mathrm{K}_{2} \mathrm{SO}_{4}, 1.25 \mathrm{mM}$ phosphoenolpyruvate (PEP), $0.25 \mathrm{mM}$ NADH (dipotassium salt), $10 \mathrm{mM} \mathrm{MgCl}_{2}, 10$ Units/mg lactate dehydrogenase (LDH), 7 Units/mg pyruvate kinase (PK), $0.01 \%$ (wt/vol) DDM and $2 \mathrm{mM} \mathrm{NaCl}$. ATP $(0.25-5 \mathrm{mM}$ ) was added 
to buffer separately in a way it is indicated below. ATP of defined concentration $(0.25-5 \mathrm{mM})$ was added to $0.5 \mathrm{ml}$ of reaction buffer and pre-incubated for 5 minutes at room temperature. In parallel, $0.5 \mathrm{ml}$ of reaction buffer (ATP-free) was pre-incubated with sample (20 $\mu \mathrm{g})$ and a substrate, silver ions in a form of silver nitrate, $20 \mu \mathrm{M}$. Absorbance was recorded in Brand ${ }^{\circledR}$ disposable plastic UV-transparent semi-micro cuvettes, at $340 \mathrm{~nm}$ using a Cary $50 \mathrm{UV}$-Vis spectrophotometer (Varian Inc). The ATP-hydrolysis reaction was initiated by addition of the reaction buffer containing pre-incubated enzyme and substrate to the reaction buffer preincubated with ATP. Activity was calculated as Units/mg ( $\mu \mathrm{M} \mathrm{ATP} / \mathrm{min} / \mathrm{mg}$ of protein) from the obtained slopes. For the determination of the $\mathrm{K}_{\mathrm{m}}$ and $\mathrm{V}_{\max }$ values, the curve fitting Michaelis-Menten function was obtained in the program Origin (Origin 8.6, OriginLab Corporation, Northampton, MA, USA) was used.

\subsubsection{Functional assay of CopA in LUVs}

CopA reconstituted into liposomes containing the $\mathrm{Ag}^{+}$-sensitive dye Phen Green SK (PGSK) was used to monitor ATP-dependent $\mathrm{Ag}^{+}$uptake. Fluorescence measurements were conducted at $25{ }^{\circ} \mathrm{C}$ with the continuous stirring using Horiba Jobin Yvon Fluorolog 3. CopA proteoliposomes (protein to lipid ratio: 1:50 (w/w); CopA concentration: $100 \mu \mathrm{g} / \mathrm{ml}$ ) or control liposomes, $100 \mu \mathrm{l}$, made in buffer $20 \mathrm{mM}$ HEPES/KOH pH 6.6, $50 \mathrm{mM} \mathrm{K}_{2} \mathrm{SO}_{4}$, containing PGSK dye $(100 \mu \mathrm{M})$, were mixed just before the measurement with $900 \mu \mathrm{l}$ assay buffer (20 mM HEPES/NaOH pH 7.6, $50 \mathrm{mM} \mathrm{K}_{2} \mathrm{SO}_{4}, 10 \mathrm{mM} \mathrm{MgSO}_{4}$ ) supplemented with 50 $\mathrm{nM}$ valinomycin and $500 \mu \mathrm{M}$ silver nitrate. The cuvette with the solution was placed in the spectrophotometer. ATP (5 mM, final concentration) was added to the sample in order to initiate the reaction. Slit width for excitation and emission $-2 \mathrm{~nm}$; excitation wavelength$480 \mathrm{~nm}$, emission wavelength- $530 \mathrm{~nm}$.

\subsubsection{Functional assay of CusA in LUVs}

CusA reconstituted into liposomes (CusA wild type or CusA D405N) were used to monitor proton-dependent $\mathrm{Ag}^{+}$uptake. Fluorescence measurements were conducted at $25{ }^{\circ} \mathrm{C}$ with the 
continuous stirring using Horiba Jobin Yvon Fluorolog 3. CusA wild type or CusA_D405N proteoliposomes (protein to lipid ratio: 1:45 (w/w); CusA concentration: $111.11 \mu \mathrm{g} / \mathrm{ml}$ ) or control liposomes, $100 \mu \mathrm{l}$, made in buffer $20 \mathrm{mM}$ HEPES/KOH pH 7.6, $50 \mathrm{mM} \mathrm{K}_{2} \mathrm{SO}_{4}$ or $20 \mathrm{mM}$ HEPES/KOH pH 6.6, $50 \mathrm{mM} \mathrm{K}_{2} \mathrm{SO}_{4}$, were mixed just before the measurement with the ACMA dye, $1 \mu \mathrm{M}$. The cuvette with the solution was placed in the spectrophotometer. In order to initiate the reaction, $900 \mu \mathrm{l}$ assay buffer $(20 \mathrm{mM}$ HEPES/KOH pH 7.6, $50 \mathrm{mM}$ $\mathrm{K}_{2} \mathrm{SO}_{4}$ or $20 \mathrm{mM}$ HEPES/KOH pH 6.6, $50 \mathrm{mM} \mathrm{K}_{2} \mathrm{SO}_{4}$ ) supplemented with $50 \mathrm{nM}$ valinomycin and $500 \mu \mathrm{M}$ silver nitrate was added to the sample. Slit width for excitation and emission $-2 \mathrm{~nm}$; excitation wavelength- $410 \mathrm{~nm}$, emission wavelength- $500 \mathrm{~nm}$.

In the case of the experiment presented in the Figure 67, Figure 68, Figure 69, proteoliposomes were diluted in $900 \mu \mathrm{l}$ assay buffer- $20 \mathrm{mM}$ HEPES/NaOH pH 6.6, $50 \mathrm{mM}$ $\mathrm{Na}_{2} \mathrm{SO}_{4}, 1 \mathrm{mM} \mathrm{AgNO}$ or $20 \mathrm{mM}$ HEPES/NaOH pH 7.6, $50 \mathrm{mM} \mathrm{Na}_{2} \mathrm{SO}_{4}, 1 \mathrm{mM} \mathrm{AgNO}$. In the case of the experiment presented in the Figure 70, PGSK dye, $100 \mu \mathrm{M}$, was reconstituted inside the vesicles. $50 \mathrm{nM}$ valinomycin was present from the beginning of the measurement in all described experiments. In the case of the experiment presented in the Figure 71 , proteoliposomes $(100 \mu \mathrm{l})$ contained $20 \mathrm{mM}$ HEPES/NaOH pH 6.6 and PGSK dye, $200 \mu \mathrm{M}$, were diluted in $900 \mu \mathrm{l}$ of buffer $20 \mathrm{mM}$ HEPES/NaOH pH 7.4 supplemented with $1 \mathrm{mM}$ $\mathrm{AgNO}_{3}$. width for excitation and emission $-2 \mathrm{~nm}$; excitation wavelength $-480 \mathrm{~nm}$, emission wavelength- $530 \mathrm{~nm}$.

\subsubsection{Drug agar plate dilution assay for testing the activity of EmrAB- TolC efflux pump}

For testing of the substrate mediated efflux by the efflux pump EmrAB-TolC, the drug agar plate dilution assay was used (Wiegand et al. 2008). Electrocompetent E. coli BW25113(DE3) $\triangle a c r A B$ cells were transformed with pRSFDMG_emrA_tolC and/or p7XC3RH_emrB (3.2.1.4). Chemically competent E. coli BW25113(DE3) $\triangle a c r A B$ were transformed with pET24a_acrB (Pos \& Diederichs, 2002) or pUC19. The E. coli BW25113 wild type strain was used as a control in comparison with E. coli BW25113(DE3) $\triangle a c r A B$ to test the sensitivity for carbonyl cyanide m-chlorophenyl hydrazone (CCCP) . When indicated, E. coli C41(DE3) wild type or C41(DE3) $\triangle a c r A B$ strains were used. Single colonies were picked to inoculate $5 \mathrm{ml} \mathrm{LB}$-medium supplemented with $50 \mu \mathrm{g} / \mathrm{ml} \mathrm{KAN}$ and/or $50 \mu \mathrm{g} / \mathrm{ml}$ 
CARB in case the cells contained plasmids with selective markers. Cultures were incubated overnight at $37{ }^{\circ} \mathrm{C}$ and $180 \mathrm{rpm}$. The overnight cultures were diluted in a series of 10 -fold steps from $\mathrm{OD}_{600} 10^{0}$ to $10^{-5}$ with fresh LB. $4 \mu$ drops of each dilution were spotted on Müller-Hinton agar plates supplemented with $20 \mu \mathrm{M}$ IPTG and the indicated CCCP concentration. Cells containing plasmids were plated on Müller-Hinton agar plates which were supplemented in addition with $50 \mu \mathrm{g} / \mathrm{ml} \mathrm{KAN} \mathrm{and/or} 50 \mu \mathrm{g} / \mathrm{ml}$ CARB. Plates were incubated overnight at $37{ }^{\circ} \mathrm{C}$. Expression levels were determined from overnight cultures grown in medium containing $50 \mu \mathrm{g} / \mathrm{ml} \mathrm{KAN,} 50 \mu \mathrm{g} / \mathrm{ml}$ CARB and $20 \mu \mathrm{M}$ IPTG. Solubilized cells samples for SDS-PAGE were prepared as described in 3.2.2.9 and proteins were identified by Western blot. 


\section{Chapter IV: Functional analysis of $E$. coli AcrAB-TolC}

\subsection{Introduction}

Even though we know structural details about each component of the pump AcrAB-TolC and in vivo analysis gave insights of some of the functional characteristics of the pump, there is still the necessity of designing in vitro assays which would make it possible to study and understand the transport mechanism of the pump (during substrate translocation), under controlled conditions. In vivo studies can have limitations, for example, the influx of the substrates can be limited in some Gram-negative bacteria across the OM barrier (Nikaido, 2009). The main advantage of in vitro assays with purified systems compared to in vivo assays is that effects from other proteins and OM limited-diffusion can be excluded. In vitro assay gives the possibility to study transporters outside the environment that can pose difficulties for manipulation.

The goal of the study presented in this thesis was to come upon a proof of principle of the method, an in vitro system, which will enable to study complex transport systems in detail. This chapter describes the basis for the in vitro assembly of AcrAB-TolC pump of E. coli. Before showing the results concerning interaction of the components of the pump reconstituted in liposomes, the chapter introduces the production of the proteins, reconstitution efficiency assays as well as activity assays of the RND component, AcrB.

As shown in Figure 22, the setup of the assay is designed in a way that one liposomal batch contains reconstituted inner membrane component AcrB and inner membrane-anchored fusion protein AcrA, while TolC is reconstituted in the other batch of liposomes. The driving force for drug extrusion by the AcrAB-TolC pump is the proton motive force (1.6.1). In this proteoliposomal system, more acidic buffer will be present outside the proteoliposomes, while a more alkaline buffer will be present inside the proteoliposomes (3.2.3.1). This creates a $\mathrm{pH}$ gradient across the membrane $(\Delta \mathrm{pH})$ directed towards the inside. The substrate of the pump will be added on the outside of the proteoliposomes. This setup would allow the antiport of $\mathrm{H}^{+} /$drugs for the $\mathrm{Acr} A B$ population that is in the right-side out orientation. 


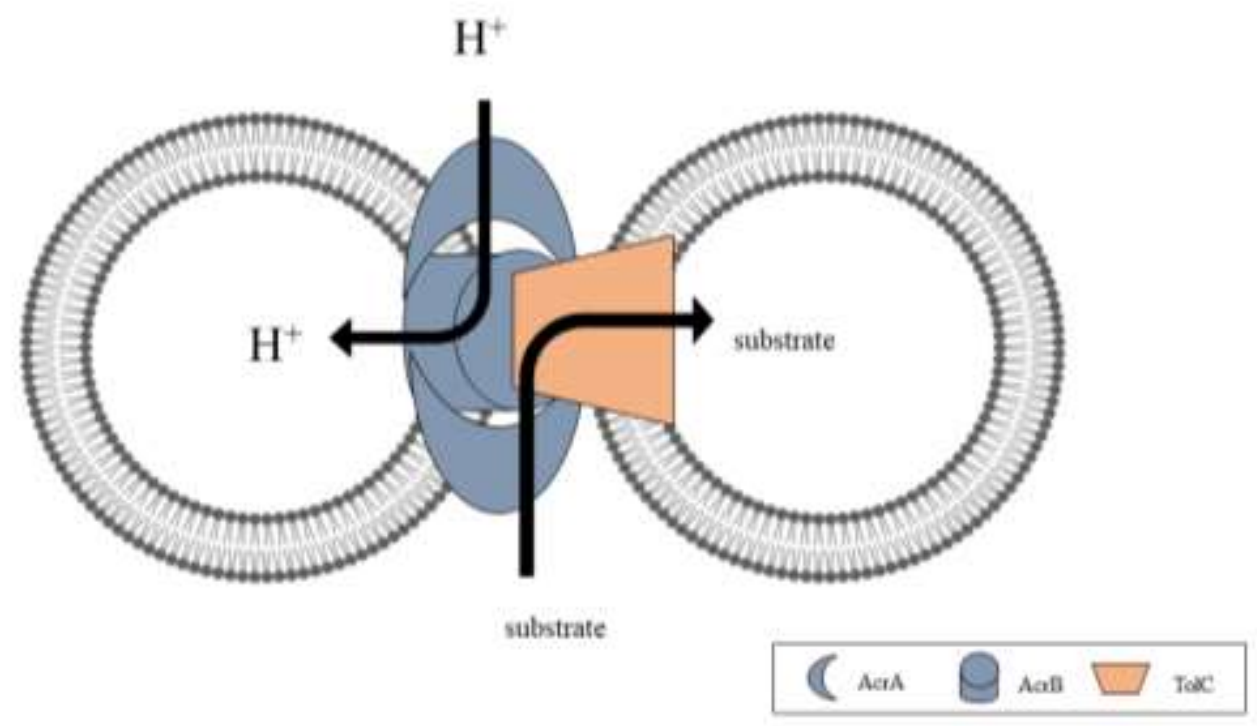

Figure 22. In vitro investigation of flux events mediated by AcrAB-TolC. AcrB and AcrA are incorporated in one liposome and TolC in another. An inward directed $\mathrm{pH}$ gradient $(\Delta \mathrm{pH}=1)$ energizes the $\mathrm{H}^{+} / \mathrm{drug}$ antiport. The drug substrate is recognized by AcrB from the outside medium as indicated. Drug will be subsequently transported into the TolC liposome.

\subsubsection{The transport of substrates results in AcrB-mediated proton influx, when AcrB is reconstituted in the right-side out orientation}

In order to establish an assay that will monitor the substrate transport mediated by the RND transporter AcrB, it is very important to know the general stability of the system, including stability of the proteoliposomes and presence of the basal $\mathrm{H}^{+}$-transport activity of the protein. For that purpose, proton transport across the membrane can be measured. In the system presented here, a $\Delta \mathrm{pH}$ (inside alkaline) is imposed, and protons are transported into the proteoliposomes. Transport of protons across the membrane can be non-carrier mediated causing acidification of the internal volume of the liposome (caused by the leakiness of the proteoliposomal membrane). Furthermore, carrier-mediated proton transport can be anticipated in the absence or presence of substrate. In the case of a tight membrane without non-carrier-mediated $\mathrm{H}^{+}$-transport, the rate of proton transport is anticipated to be faster in the presence of AcrB substrate (added to the outside), since AcrB couples the influx of protons with the binding and release of drug molecules. To monitor the acidification of the (weak) buffer on the inside of the AcrAB-containing liposomes, the pH-sensitive dye, 
pyranine is incorporated into the proteoliposomes during the reconstitution procedure and its fluorescence (excitation wavelength- $460 \mathrm{~nm}$; emission wavelength - $510 \mathrm{~nm}$ ) was measured over time (Figure 23).
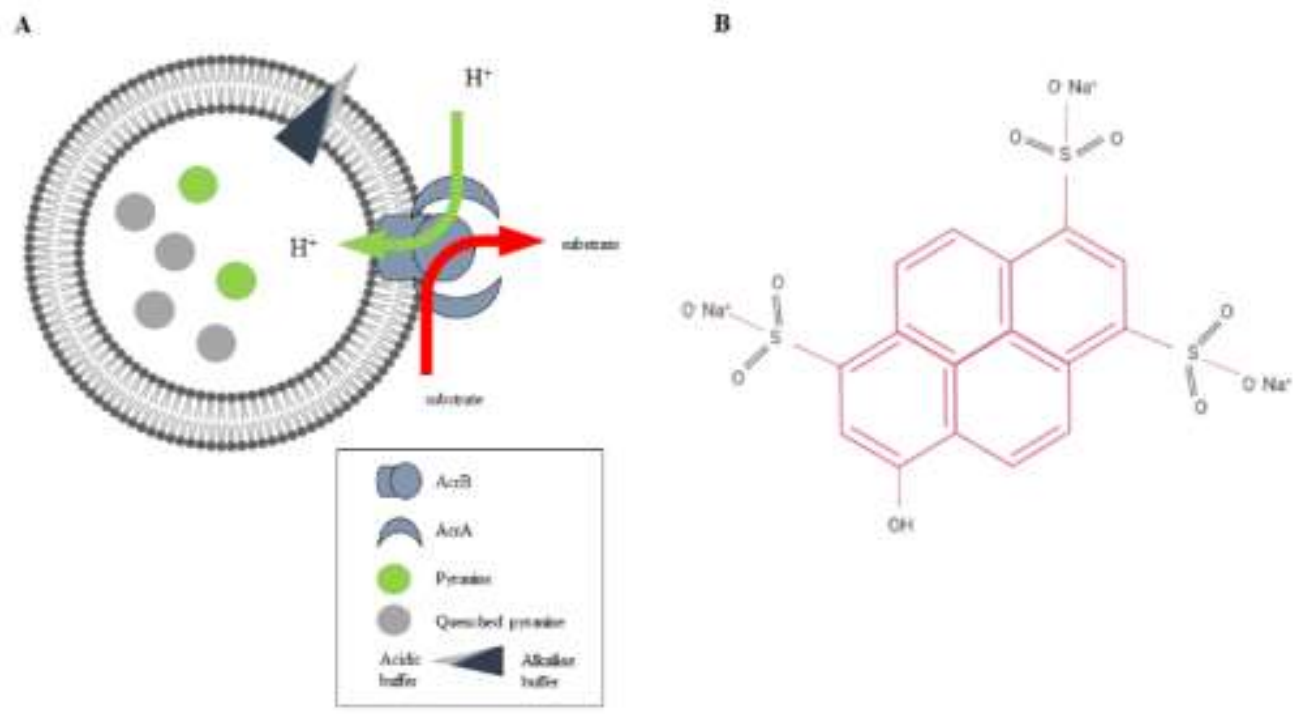

Figure 23. Blueprint of an RND efflux pump assay. A. Experimental setup. A pH-sensitive dye, 8-hydroxy-1,3,6-pyrenetrisulfonate (pyranine), is used to monitor the acidification of the lumen of the proteoliposome that contains reconstituted AcrB or AcrAB. By diluting proteoliposomes into the buffer with lower $\mathrm{pH}$ compared to the $\mathrm{pH}$ inside the proteoliposomes, a $\Delta \mathrm{pH}$ (alkaline inside) is imposed across the membrane. Upon proton-transport into the proteolipomes, the internal buffer volume acidifies, resulting in pyranine fluorescence quenching. B. Structure of pyranine.

\subsection{Results}

\subsubsection{Pyranine as a reporter system for observations of proton flux in the proteoliposome assay}

Pyranine is a dye that is highly soluble in water, due to presence of three sulfonate groups in its structure (Figure 23B). The dye can be incapsulated inside the liposomes during reconstitution (Verchère et al., 2015). Pyranine that is not incapsulated inside the liposomes can be removed by washing (centrifugation/resuspension) and/or by gel filtration of the proteoliposomes. In this work, external pyranine was removed via passing the sample over a NAP10-desalting column followed by several iterations of centrifugation (washing/resuspension steps). Pyranine does not leak out of the liposomes spontaneously and 
does not bind to the membrane of the liposomes at the conditions used (Kano \& Fendler, 1978). Transport of protons into the proteoliposomes and thus monitoring acidification of their lumen through quenching of the dye, is a reliable way to monitor the activity of the inner membrane protein, AcrB.

Several features of pyranine can be readily used to indirectly monitor the drug transport activity of AcrB transporter: 1) The ability of the dye to be exclusively incorporated into the liposomes; by removal of the dye on the outside of the proteoliposomes; 2) Pyranine does not spontaneously leak out the liposome, and does not interact with the membrane; 3) Pyranine quenches upon acidification, which is the important feature for studying the $\mathrm{H}^{+}$-transport activity of $\mathrm{H}^{+}$-dependent RND antiporters.

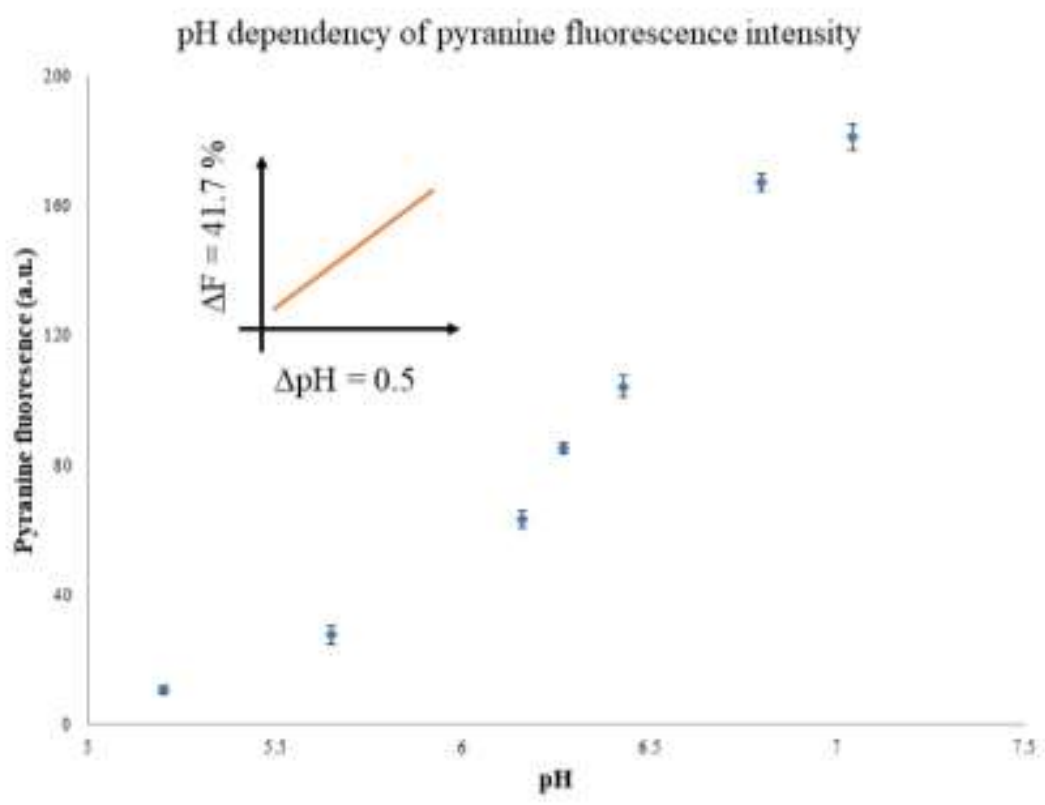

Figure 24. Fluorescent signal of pyranine in dependence of the pH. Pyranine $(0.5 \mathrm{mM})$ was tested in a buffer $(20 \mathrm{mM}$ HEPES) at different $\mathrm{pH}$ values, in a range of $\mathrm{pH} 5.2$ to $\mathrm{pH} 7.04$. Fluorescence was measured at $\lambda_{\mathrm{ex}}=460 \mathrm{~nm} ; \lambda_{\mathrm{em}}=510 \mathrm{~nm}$ and $\mathrm{F}$ at $510 \mathrm{~nm}$ is shown. Each point is average of three consecutive measurements $(\mathrm{n}=3)$. Inset: Linear correlation between pyranine fluorescence and $\mathrm{pH}$, between $\mathrm{pH} 5.2$ and $\mathrm{pH} 7.04, \Delta \%$ fluorescence $\mathrm{F}=99.157 \mathrm{x} \Delta \mathrm{pH}-525.56\left(\mathrm{R}^{2}=0.9387\right)$.

Figure 24 shows the linear dependence between $\mathrm{pH}$ and fluorescence intensity of pyranine (here shown as fluorescence intensity at $510 \mathrm{~nm}$ ). This linear dependence is especially important since the changes in the fluorescence signal can be correlated with the changes in the $\mathrm{pH}$ inside the proteoliposomes. This provides insights into the proton transport activities. 


\subsubsection{Production of proteins for in vitro assays}

Production of highly stable and pure proteins was performed in E. coli C43(DE3) $\triangle a c r A B D$ as described in 3.2.2.2. In the sections below the profiles of the Size Exclusion Chromatography (SEC) for each purified protein is shown, as well as the purity levels and possible degradation products present in the sample.

\subsubsection{Production of AcrA A200V}

The AcrA variant, A200V (AcrA A200V) was used in this study, since it had been discovered that this variant gives better yield of production then the AcrA wildtype, without affecting the phenotype (Dr. Ravi KR Marreddy, personal communication). For the purpose of production, the gene encoding AcrA A200V was cloned into the p7XC3H vector and expressed in E. coli C43(DE3) $\triangle a c r A B D$ as a C-terminal His-tagged version using IPTG $(0.5 \mathrm{mM})$ as an inducer. As shown in Figure 25, AcrA A200V was purified in a two-step procedure, using $\mathrm{Ni}^{2+}$-NTA system and Size Exclusion Chromatography (SEC). Purification was facilitated by ion metal affinity chromatography (IMAC) employing $\mathrm{Ni}^{2+}-\mathrm{NTA}$ resins. Detergent $\mathrm{n}$-dodecyl- $\beta$-maltoside (DDM) was used for membrane solubilization and membrane protein purification. The last step of purification was Size Exclusion Chromatography (SEC) so that aggregates and low molecular weight contaminants are removed. The SEC profile indicated highly pure and monodisperse protein. AcrA A200V was present as a dimer, as concluded from the elution volume in the SEC, as well as from Blue Native PAGE (BN-PAGE) analysis. Protein yield amounted to approximately 1.5 - $2 \mathrm{mg}$ of purified AcrA A200V after SEC per litre of culture. 
A

B
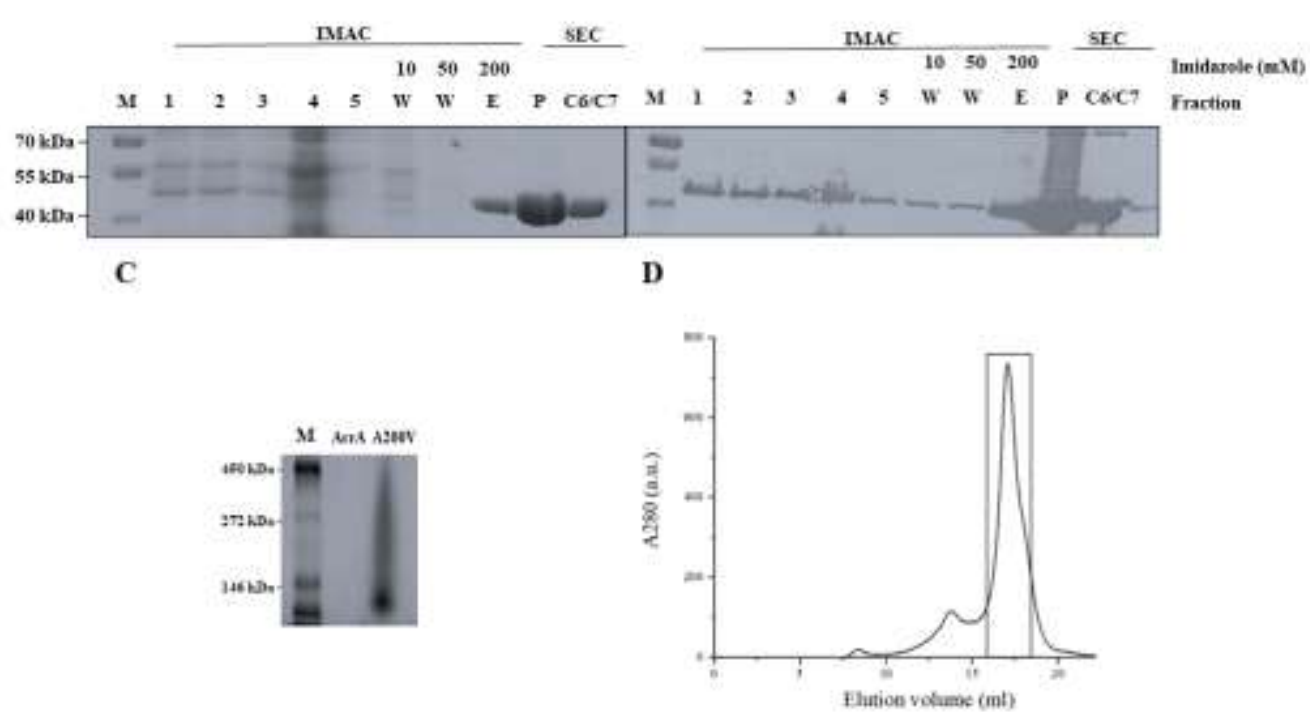

Figure 25. Characterization of the purity, monodispersity and oligomeric state of AcrA A200V. A. Coomassie-stained $10 \%$ SDS-PAA gel of the purification steps toward purified AcrA A200V. B. Western Blot analysis with identical samples from A. The Western Blot was probed with monoclonal anti-polyHistidine-alkaline phosphatase produced in mouse (Sigma A5588). M-Marker (PageRuler Prestained Protein Ladder (ThermoScientific)), 1-Membrane, 2-Solubilized membrane, 3Supernatant after ultracentrifugation of the solubilized membrane sample, 4-Pellet after ultracentrifugation of the solubilized membrane sample, 5-Flow through of the supernatant sample from 3 after application on a $\mathrm{Ni}^{2+}$-NTA-column, W-Samples from indicated (buffer with $10 \mathrm{mM}$ imidazole or $50 \mathrm{mM}$ imidazole) wash steps from $\mathrm{Ni}^{2+}$-NTA purification, E-Eluate, PConcentrated protein sample after $\mathrm{Ni}^{2+}$-NTA purification, C6/C7-Samples from the fractions collected after SEC. Applied: 1-P (samples were diluted 12.5x in SDS-sample buffer) $10 \mu \mathrm{l}$ for Coomassie, $5 \mu 1$ for Western blot analysis; P- $3 \mu \mathrm{g}$ for Coomassie, $3 \mu \mathrm{g}$ for Western blot; C6/C7- $2 \mu \mathrm{g}$ for Coomassie, $2 \mu \mathrm{g}$ for Western blot. C. Purified AcrA A200V (12 $\mu \mathrm{g})$ was loaded on 4-20\% Mini-PROTEAN TGX Precast Protein Gels (Bio-Rad). 4-20\% Mini-PROTEAN TGX Precast Protein Gels $($ Bio-Rad). Molecular weight marker = NativeMark Unstained Protein Ladder (Thermo Fisher). D. Size Exclusion Chromatography profile of a $\mathrm{Ni}^{2+}$-NTA purified sample of AcrA A200V. The protein was purified on a Superose 6 Increase $30 / 100$ column equilibrated with $20 \mathrm{mM}$ Tris/Cl, $\mathrm{pH} 7.5,150 \mathrm{mM} \mathrm{KCl}, 0.03 \% \mathrm{DDM}$, with a flow rate of $0.4 \mathrm{ml} / \mathrm{min}$. The peak was detected at $17 \mathrm{ml}$.

\subsubsection{Purification of AcrB wild type and AcrB D407N}

Production of highly stable and pure AcrB with 10xHis tag was successfully performed. The expression in E. coli C43(DE3) $\triangle a c r A B D$ with a C-terminal His-tagged version using IPTG $(0.5 \mathrm{mM})$ as an inducer was performed as described in 3.2.2.1. Expression and purification are adapted from Pos and Diederichs (Pos \& Diederichs, 2002). The AcrB wild type protein was purified as a trimer. The oligomeric state was confirmed by SEC which yielded a monodisperse peak at the elution volume corresponding to the molecular weight of the trimeric RND, as well as by BN-PAGE analysis. The purity was confirmed by SDS-PAGE analysis and Coomassie staining and via Western Blot analysis (Figure 26). The yield of pure and monodisperse AcrB wildtype protein was approximately $1-1.5 \mathrm{mg}$ per litre of culture. 
A

B

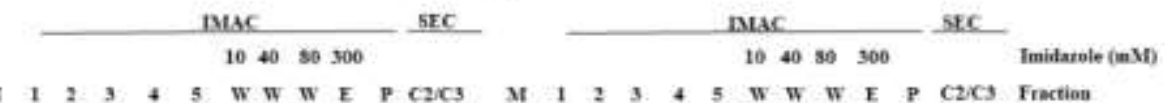

$\mathrm{M} 123+5 \mathrm{~W}$ W $\mathrm{W}$ P $\mathrm{C}_{2} \mathrm{Cl}_{3}$

M 123.45 W W W E C2/CS Fraction

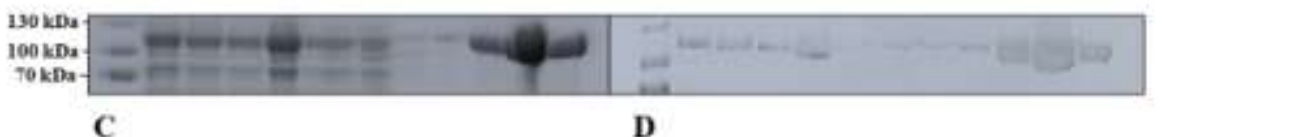

C

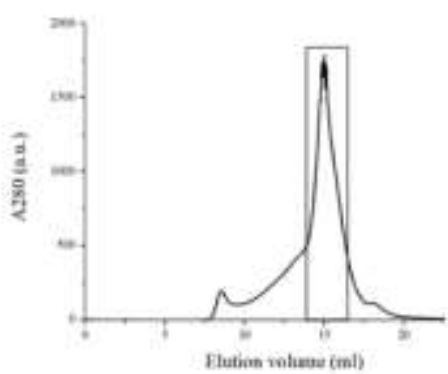

Figure 26. Characterization of the purity, monodispersity, and oligomeric state of AcrB wild type. A. Coomassie-stained $10 \%$ SDS-PAA gel of the purification steps toward purified AcrB. B. Western Blot analysis with identical samples from A. The Western Blot was probed with monoclonal anti-polyHistidine-alkaline phosphatase produced in mouse (Sigma A5588). M-Marker (PageRuler Prestained Protein Ladder (ThermoScientific)), 1-Membrane, 2-Solubilized membrane, 3Supernatant, 4-Pellet, 5-Flow through, W-Samples from indicated wash steps from $\mathrm{Ni}^{2+}$-NTA purification, E-Eluate, PConcentrated protein sample after $\mathrm{Ni}^{2+}$-NTA purification, C2/C3-Samples from the fractions collected after SEC. Applied: 1-P (samples were diluted 12.5x in SDS-sample buffer) $10 \mu \mathrm{l}$ for Coomassie, $5 \mu \mathrm{l}$ for Western blot; P- $5 \mu \mathrm{g}$ for Coomassie, 2 $\mu \mathrm{g}$ for Western blot; C2/C3- $3 \mu \mathrm{g}$ for Coomassie, $1 \mu \mathrm{g}$ for Western blot. C. Purified AcrB (18 $\mu \mathrm{g}$ ) was loaded on 4-20\% Mini-PROTEAN TGX Precast Protein Gels (Bio-Rad). 4-20 \% Mini-PROTEAN TGX Precast Protein Gels (Bio-Rad). Molecular weight marker $=$ NativeMark Unstained Protein Ladder (Thermo Fisher). D. Size Exclusion Chromatography profile of a $\mathrm{Ni}^{2+}$-NTA purified sample of AcrB. The protein was purified on a Superose 6 Increase 30/100 column equilibrated with $20 \mathrm{mM}$ Tris/Cl pH 7.5, $150 \mathrm{mM} \mathrm{KCl}, 0.02 \% \mathrm{DDM}$, with a flow rate of $0.4 \mathrm{ml} / \mathrm{min}$. The peak was detected at $14.9 \mathrm{ml}$.

The AcrB D407N variant was purified with the same conditions for the AcrB wild type and with the same purity and oligomerization state (shown on Figure 27C). Protein production usually gave around $2.6 \mathrm{mg}$ from $10 \mathrm{ml}$ membrane. For the purpose of production, membrane aliquot was provided with courtesy by Andrea Herrmann. 


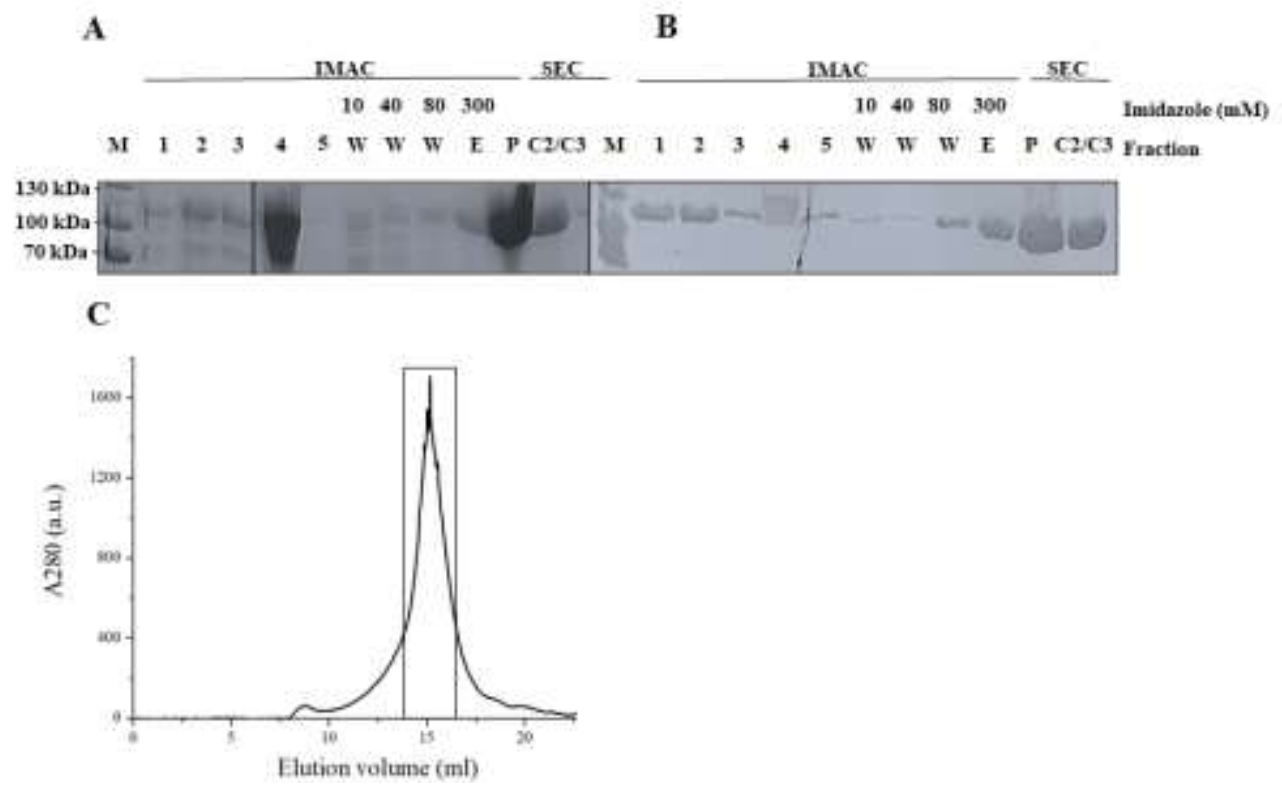

Figure 27. Characterization of the purity, monodispersity and oligomeric state of AcrB D407N. A. Coomassie-stained $10 \%$ SDS-PAA gel of the purification steps toward purified AcrB D407N. B. Western Blot analysis with identical samples from A. The Western Blot was probed with monoclonal anti-polyHistidine-alkaline phosphatase produced in mouse (Sigma A5588). M-Marker (PageRuler Prestained Protein Ladder (ThermoScientific)), 1-Membrane, 2-Solubilized membrane, 3Supernatant, 4-Pellet, 5-Flow through, W-Samples from indicated wash steps from $\mathrm{Ni}^{2+}$-NTA purification, E-Eluate, PConcentrated protein sample after $\mathrm{Ni}^{2+}$-NTA purification, C2/C3-Samples from the fractions collected after SEC. Applied: 1-P (samples were diluted 12.5x in SDS-sample buffer) $10 \mu \mathrm{l}$ for Coomassie, $5 \mu \mathrm{l}$ for Western blot; P- $6 \mu \mathrm{g}$ for Coomassie, 2 $\mu \mathrm{g}$ for Western blot; C2/C3- $2 \mu \mathrm{g}$ for Coomassie, $2 \mu \mathrm{g}$ for Western blot. C. Size Exclusion Chromatography profile of a $\mathrm{Ni}^{2+}$-NTA purified sample of AcrB D407N. The protein was purified on a Superose 6 Increase 30/100 column equilibrated

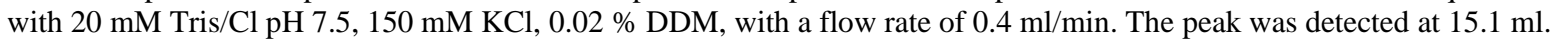

\subsubsection{Production of TolC wild type}

The expression of TolC in E. coli C43(DE3) $\triangle a c r A B D$ with a C-terminal His-tagged version using IPTG $(0.5 \mathrm{mM})$ as an inducer was performed as described in 3.2.2.1. TolC wild type was purified as a trimer, shown by Blue Native PAGE analysis and SEC (Figure 28). The trimer peak was detected at $15.3 \mathrm{ml}$. However, some traces of monomer are present in the sample after $\mathrm{Ni}^{2+}$-NTA purification and some additional "shoulder peak" is visible on the SEC. Looking at the elution volume of the shoulder peak of around $17 \mathrm{ml}$, this should correspond to the monomer of TolC. An additional band of a TolC monomer is visible on the Western blot lane with a sample after SEC. The sample of purified TolC contains besides the trimeric TolC also some minor fraction of the monomer present. Protein production usually gave around $0.5 \mathrm{mg}$ per litre of culture. 


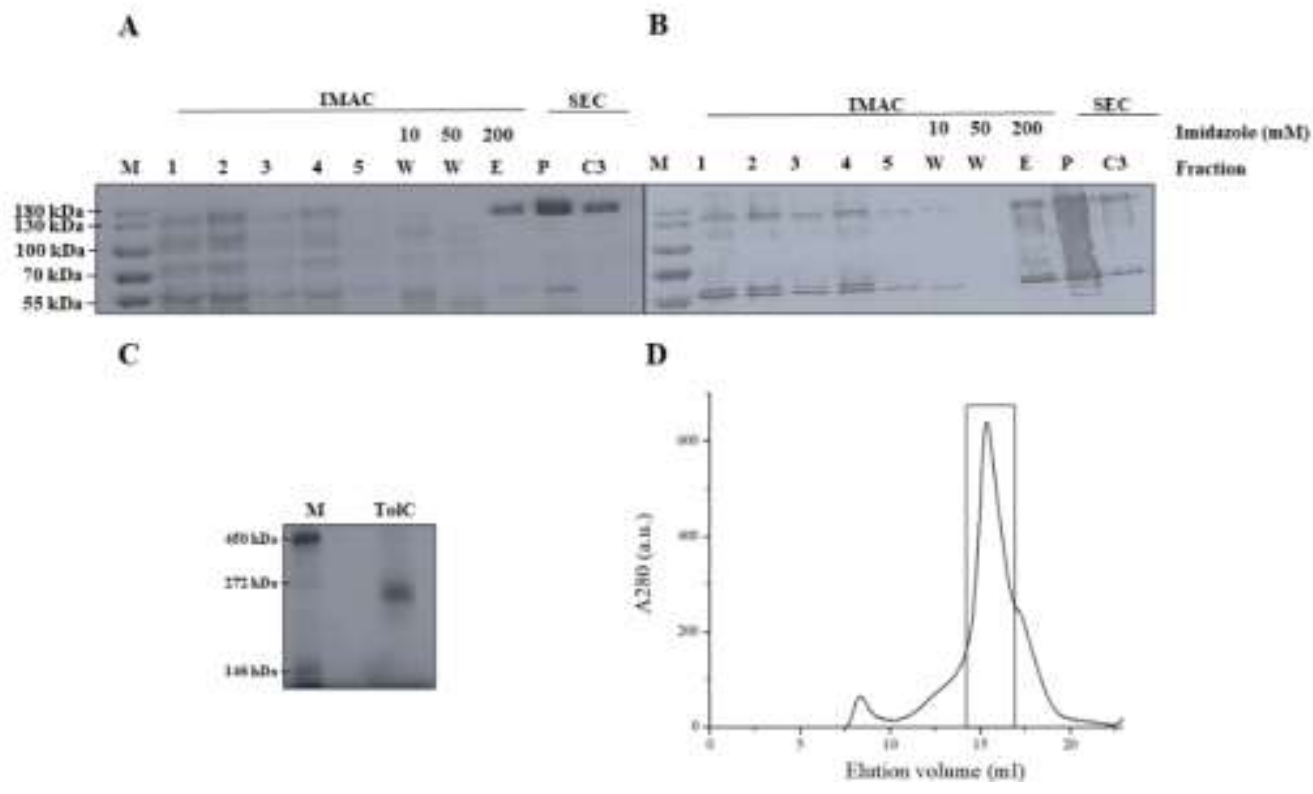

Figure 28. Characterization of the purity, monodispersity and oligomeric state of TolC wild type. A. Coomassie-stained $10 \%$ SDS-PAA gel of the purification steps toward purified TolC. B. Western Blot analysis with identical samples from A. The Western Blot was probed with monoclonal anti-polyHistidine-alkaline phosphatase produced in mouse (Sigma A5588). M-Marker (PageRuler Prestained Protein Ladder (ThermoScientific)), 1-Membrane, 2-Solubilized membrane, 3Supernatant, 4-Pellet, 5-Flow through, W-Samples from indicated wash steps from $\mathrm{Ni}^{2+}$-NTA purification, E-Eluate, PConcentrated protein sample after $\mathrm{Ni}^{2+}$-NTA purification, C2/C3-Samples from the fractions collected after SEC. Applied: 1-E (samples were diluted 12.5x in SDS-sample buffer) $10 \mu \mathrm{l}$ for Coomassie, $5 \mu \mathrm{l}$ for Western blot; P- $2 \mu \mathrm{g}$ for Coomassie, 2 $\mu \mathrm{g}$ for Western blot; C3- $1.5 \mu \mathrm{g}$ for Coomassie, $1 \mu \mathrm{g}$ for Western blot. C. Purified TolC (6.4 $\mu \mathrm{g})$ was loaded on 4- $20 \%$ Mini-PROTEAN TGX Precast Protein Gels (Bio-Rad). 4-20\% Mini-PROTEAN TGX Precast Protein Gels (Bio-Rad). Molecular weight marker $=$ NativeMark Unstained Protein Ladder (Thermo Fisher). D. Size exclusion chromatography profile of a $\mathrm{Ni}^{2+}$-NTA purified sample of AcrB. The protein was purified on a Superose 6 Increase 30/100 column

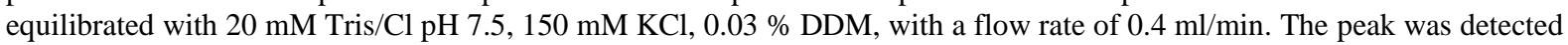
at $15.3 \mathrm{ml}$ corresponding to the trimer of TolC.

\subsubsection{The blueprint of the proteoliposome assay - detection of periplasmic drug transport by AcrB}

AcrB wild type was reconstituted in Cholesterol/DOPC (1:3 ratio, w/w) liposomes, via detergent-mediated reconstitution. The assay was performed as described in 3.2.3.1. Proteoliposomes containing AcrB wild type (buffer inside the proteoliposome $-10 \mathrm{mM}$

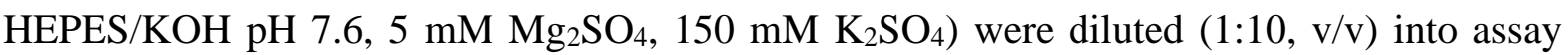
buffer - $10 \mathrm{mM}$ HEPES/KOH pH 6.6, $5 \mathrm{mM} \mathrm{Mg}_{2} \mathrm{SO}_{4}, 150 \mathrm{mM} \mathrm{K}_{2} \mathrm{SO}_{4}$, containing $1 \mathrm{mM}$ taurocholate (sodium salt), a substrate of the AcrB pump. Dilution into the assay buffer was done to start the reaction, $\mathrm{t}=0$ seconds. DOPC, which is used for liposome preparation is zwitterionic, while cholesterol is neutral. $5 \mathrm{mM} \mathrm{MgCl} 2$ was added to the buffers since $\mathrm{Mg}^{2+}$ 
stabilizes the lipid membrane surface by interacting with the negative phosphates of the lipids. $2 \mathrm{mM}$ pyranine is incorporated inside the liposomes.

A

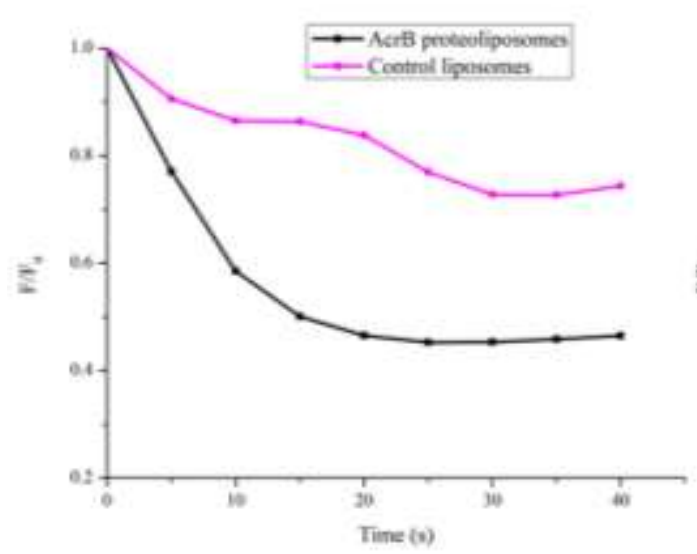

B

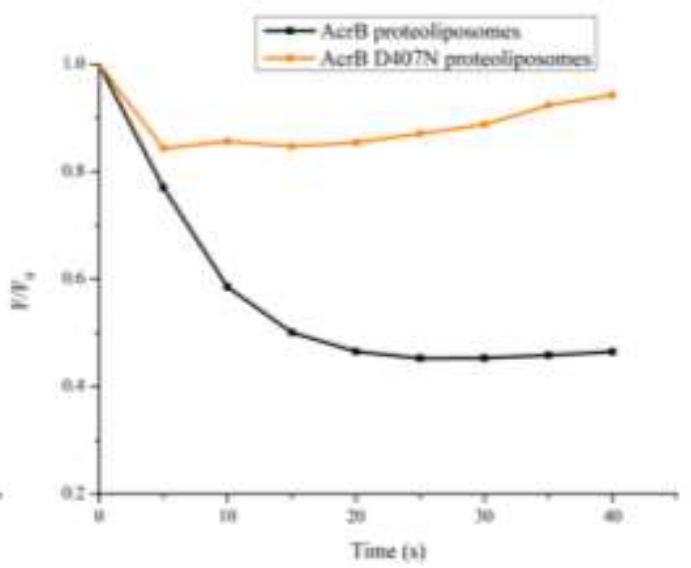

Figure 29. $\mathrm{H}^{+}$-transport in $\mathrm{AcrB}$ proteoliposomes and non-protein containing liposomes. A. $\mathrm{H}^{+}$influx into AcrB proteoliposomes compared to the $\mathrm{H}^{+}$influx into control liposomes (liposomes without protein incorporated). B. $\mathrm{H}^{+}$influx into AcrB proteoliposomes compared to $\mathrm{H}^{+}$influx into AcrB D407N-containing proteoliposomes. The AcrB proteoliposomes trace in $\mathrm{A}$ is identical with the one in $\mathrm{B}$. The change of fluorescence $\left(\mathrm{F} / \mathrm{F}_{0}\right)$ of the $\mathrm{pH}$ sensitive dye, pyranine, was monitored over time. At the $\mathrm{t}=0$ seconds, the (proteo)liposomes containing buffer at $\mathrm{pH} 7.6$, were diluted 1:10 (v/v) into the same buffer at $\mathrm{pH} 6.6$, imposing a $\Delta \mathrm{pH}$ of 1 across the membrane. The buffer with a $\mathrm{pH} 6.6$ was supplemented with the substrate, taurocholate (sodium salt, $1 \mathrm{mM}$ ). Fluorescence values were normalized using $F / F_{0}$, where $F_{0}$ is the initial value at $\mathrm{t}=0$ seconds. Fluorescence was measured at $\lambda_{\mathrm{ex}}=460 \mathrm{~nm} ; \lambda_{\mathrm{em}}=510 \mathrm{~nm}$.

As shown in Figure 29, pyranine fluorescence is quenched $\left(\mathrm{F} / \mathrm{F}_{0}\right.$ approximately 0.45 at $\mathrm{t}=40$ seconds) in the sample with AcrB wild type proteoliposomes in the presence of taurocholate. The liposome sample without incorporated protein does only show moderate quenching ( $\mathrm{F} / \mathrm{F}_{0}$ approximately 0.8 at $\mathrm{t}=40$ seconds), most likely due to non-carrier mediated proton leakage through the membrane, that can be induced by interactions of hydrophobic substrates with the liposome membrane.

Except the control liposomes (empty liposomes, no protein incorporated), the assay was performed with vesicles containing a proton-transport deficient variant, AcrB D407N. Previously it has been shown that this variant has a mutation that targets the proton relay network, which influences the transport capability of AcrB as a proton antiporter (Markus A. Seeger et al., 2009). With this variant present in the liposomes, pyranine fluorescence quenching was comparable in rate and extent as with liposomes without reconstituted protein, indicating that the observed quenching is most likely due to non-carrier mediated influx of 
protons. The AcrB D407N variant is therefore a good negative control for the further $\mathrm{H}^{+}$ transport studies (Figure 29).

As shown by Zgurskaya and Nikaido (H. I. Zgurskaya \& Nikaido, 1999), when soluble (nonmembrane anchored) AcrA was used in the assay in the presence of $\mathrm{MgCl}_{2}, \mathrm{AcrB}$-dependent $\mathrm{H}^{+}$-transport activity was greatly stimulated. To test the influence of membrane-anchored AcrA in the system presented here, AcrA was co-reconstituted with AcrB. Proteoliposomes ( $\mathrm{pH}$ inside 7.6) were diluted into buffer with $\mathrm{pH} 6.6$ to impose a $\mathrm{pH}$ gradient. A substrate of the pump, taurocholate (sodium salt, $1 \mathrm{mM}$ ), was present in the assay buffer. Proteoliposomes including $\mathrm{Acr} A \mathrm{~B}$, showed a faster pyranine quenching rate and final quenching after 5 minutes compared to the experiment with non-protein-containing liposomes under equivalent conditions (Figure 30).

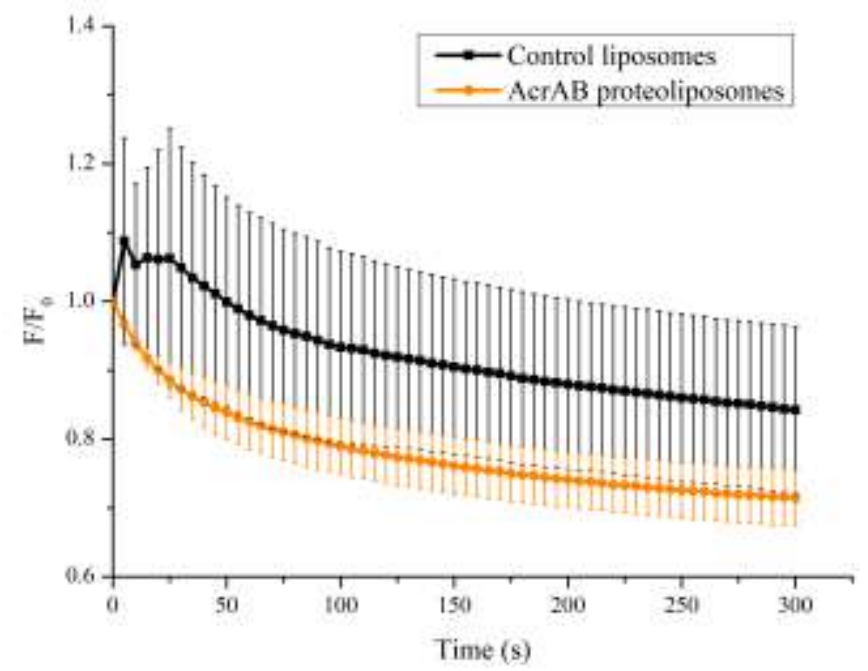

Figure 30. $\mathrm{H}^{+}$-transport in $\mathrm{Acr} A \mathrm{~B}$ proteoliposomes and non-protein containing liposomes. The change of fluorescence $\left(\mathrm{F} / \mathrm{F}_{0}\right)$ of the $\mathrm{pH}$ sensitive dye, pyranine, was monitored over time. At the $\mathrm{t}=0$ seconds, the (proteo)liposomes containing buffer at $\mathrm{pH} 7.6$, were diluted $1: 10(\mathrm{v} / \mathrm{v})$ into the same buffer at $\mathrm{pH} 6.6$, imposing a $\Delta \mathrm{pH}$ of 1 across the membrane. The buffer with a $\mathrm{pH} 6.6$ was supplemented with the substrate, taurocholate (sodium salt, $1 \mathrm{mM}$ ). Fluorescence values were normalized using $\mathrm{F} / \mathrm{F}_{0}$, where $\mathrm{F}_{0}$ is the initial value at $\mathrm{t}=0$ seconds. Fluorescence was measured at $\lambda \mathrm{ex}=460 \mathrm{~nm} ; \lambda \mathrm{em}=510 \mathrm{~nm}$. Each point is an average of three separate measurements $(n=3)$.

In contrast with the research previously done (H. I. Zgurskaya \& Nikaido, 1999), this study shows that when AcrA was co-reconstituted with AcrB in liposomes, AcrAB proton pumping activity seems not to be influenced; the pyranine signal decreases since the protons that enter the liposomes quench the fluorescence of the dye (Figure 31, black curve). The decrease in 
fluorescence is similar to the decrease observed for AcrB proteoliposomes (Figure 31, blue curve). This observation was made in comparison with the empty vesicles as well (Figure 30). The difference in the fluorescence quenching values $\left(\mathrm{F} / \mathrm{F}_{0}\right)$ between Figure $29\left(\mathrm{~F} / \mathrm{F}_{0}=\right.$ 0.45, $\mathrm{t}=40$ seconds $)$ and Figure $30\left(\mathrm{~F} / \mathrm{F}_{0}=0.85, \mathrm{t}=40\right.$ seconds $)$ are notable, and they most likely come from the fact that reconstitution success as well as activity of the protein are batch to batch dependent hence the quenching might vary between two samples from two different reconstitution experiments.

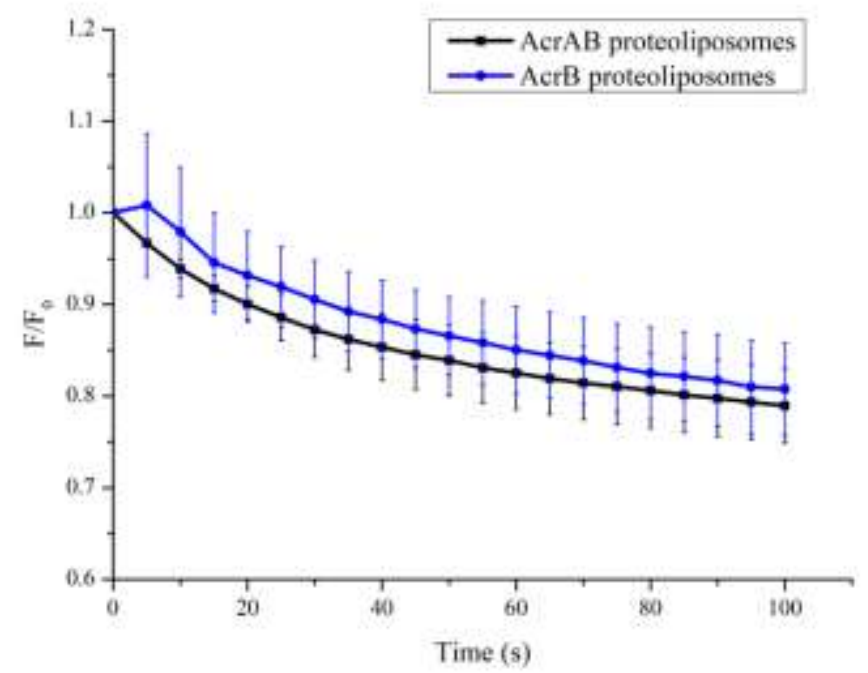

Figure 31. $\mathrm{H}^{+}$-transport in $\mathrm{AcrB}$ and $\mathrm{Acr} A \mathrm{~B}$ proteoliposomes. The change of fluorescence $\left(\mathrm{F} / \mathrm{F}_{0}\right)$ of the $\mathrm{pH}$ sensitive dye, pyranine, was monitored over time. At the $\mathrm{t}=0$ seconds, the (proteo)liposomes containing buffer at $\mathrm{pH}$ 7.6, were diluted 1:10 $(\mathrm{v} / \mathrm{v})$ into the same buffer at $\mathrm{pH} 6.6$, imposing a $\Delta \mathrm{pH}$ of 1 across the membrane. The buffer with a $\mathrm{pH} 6.6$ was supplemented with the substrate, taurocholate (sodium salt, $1 \mathrm{mM}$ ). Fluorescence values were normalized using $\mathrm{F} / \mathrm{F}_{0}$, where $\mathrm{F}_{0}$ is the initial value at $\mathrm{t}=0$ seconds. Fluorescence was measured at $\lambda \mathrm{ex}=460 \mathrm{~nm} ; \lambda \mathrm{em}=510 \mathrm{~nm}$. Each point is an average of three separate measurements $(n=3)$.

The presented data indicate that, under the current experimental conditions, AcrA does not stimulate the activity of AcrB as it was shown for the homologues of AcrA and AcrB, from P. aeruginosa, MexA and MexB, respectively (Verchère et al., 2012).

When AcrA was co-reconstituted with AcrB D407N, no quenching of pyranine fluorescent signal was observed, indicating that proton transport is not taking place (olive curve, Figure 32). The data was compared with the data of AcrB wild type and the inactive variant, AcrB D407N as well as with control liposomes containing no protein. All data taken for comparison are the same as in Figure 29. 


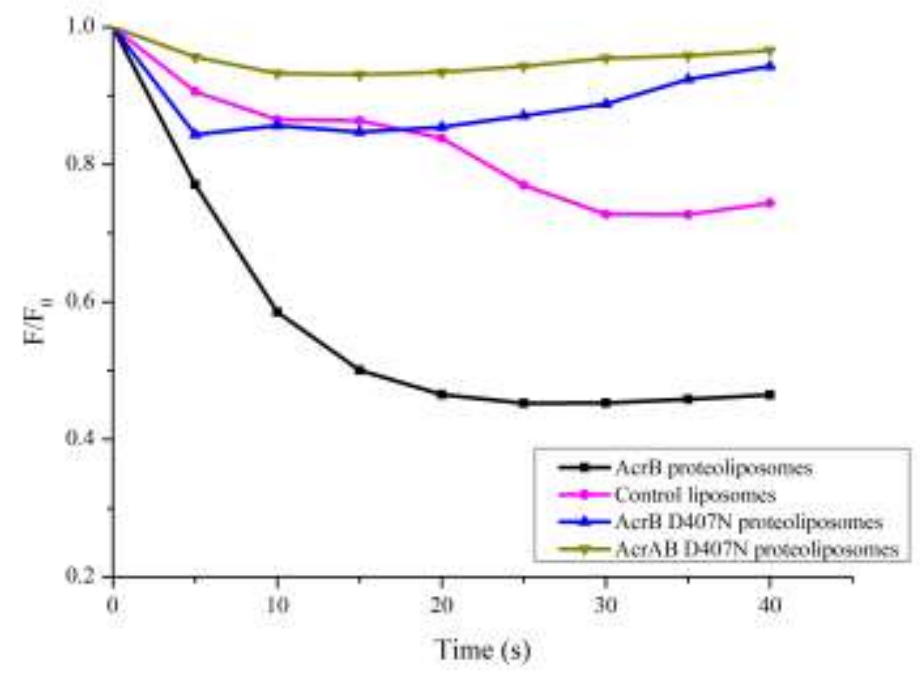

Figure 32. AcrA does not restore the activity of AcrB D407N. $\mathrm{H}^{+}$-transport in AcrB, AcrB D407N, AcrAB D407N and nonprotein containing (proteo)liposomes. The change of fluorescence $\left(\mathrm{F} / \mathrm{F}_{0}\right)$ of the $\mathrm{pH}$ sensitive dye, pyranine, was monitored over time. At the $\mathrm{t}=0$ seconds, the (proteo)liposomes containing buffer at $\mathrm{pH} 7.6$, were diluted 1:10 (v/v) into the same buffer at $\mathrm{pH} 6.6$, imposing a $\Delta \mathrm{pH}$ of 1 across the membrane. The buffer with a $\mathrm{pH} 6.6$ was supplemented with the substrate, taurocholate (sodium salt, $1 \mathrm{mM}$ ). Fluorescence values were normalized using $F / F_{0}$, where $F_{0}$ is the initial value at $\mathrm{t}=0$ seconds. Fluorescence was measured at $\lambda_{\mathrm{ex}}=460 \mathrm{~nm} ; \lambda_{\mathrm{em}}=510 \mathrm{~nm}$.

\subsubsection{The activity of AcrB in the presence of MBX3132 and Phe-Arg $\beta$ - naphthylamide (PAßN)}

The usage of the known AcrB inhibitor MBX3132 (Figure 33) together with the substrate taurocholate (sodium salt) increases the quenching of pyranine fluorescence $\left(F / F_{0}=0.8\right.$, $\mathrm{t}=40$ seconds $)$ compared to experiments without inhibitor $\left(\mathrm{F} / \mathrm{F}_{0}=0.85, \mathrm{t}=40\right.$ seconds $)$.

In the experiment shown in Figure 34, AcrB and AcrAB proteoliposomes were used. The quenching of pyranine fluorescence in $\mathrm{Acr} A \mathrm{~B}$ proteoliposomes is comparable with the quenching of pyranine fluorescence in AcrB proteoliposomes $\left(\mathrm{F} / \mathrm{F}_{0}=0.90\right.$ and $\mathrm{F} / \mathrm{F}_{0}=0.85$ at $\mathrm{t}=40$ seconds, for AcrB and AcrAB proteoliposomes, respectively). MBX3132 was added together with taurocholate at the beginning of the measurement. Even with MBX3132, slopes look similar, except for the first seconds, when the slope is slightly steeper. 


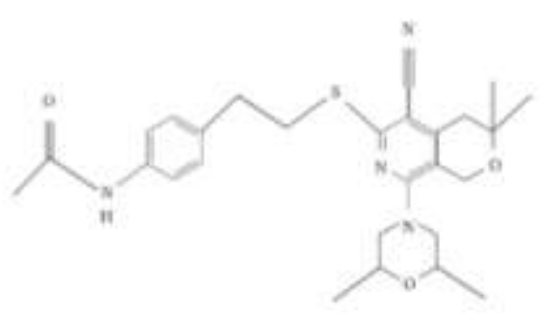

$\operatorname{MBX} 3132$

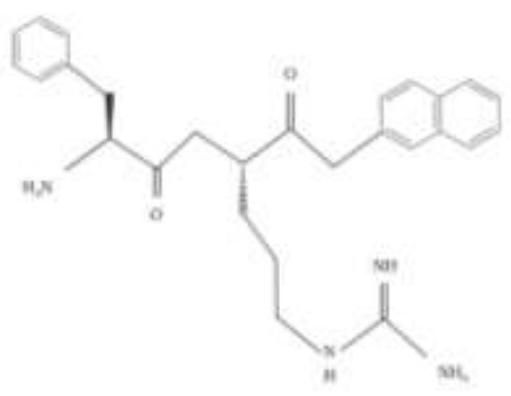

PABN

Figure 33. Structures of AcrB inhibitors, MBX3132 and Phe-Arg- $\beta$-naphthylamide (PA $\beta N$; MC-207,110). Figure was made with help of BioRender (https://app.biorender.com/).

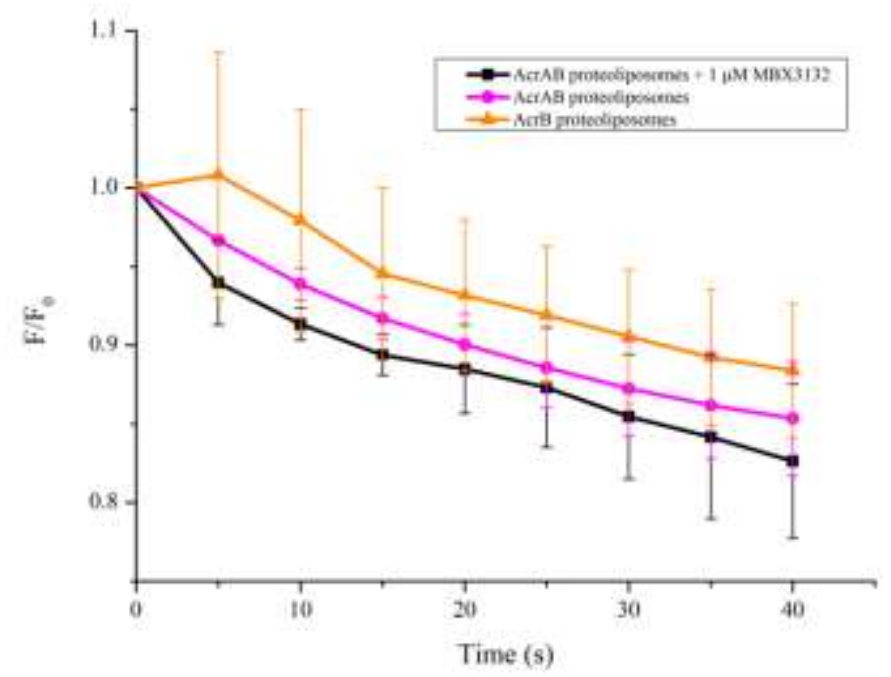

Figure 34. $\mathrm{H}^{+}$-transport in $\mathrm{AcrB}$ and $\mathrm{Acr} A \mathrm{~B}$ proteoliposomes. AcrB and AcrAB proteoliposomes are tested in the presence of substrate (taurocholate) and AcrAB proteoliposomes in the presence of substrate (taurocholate) and inhibitor (MBX3132). The change of fluorescence $\left(\mathrm{F} / \mathrm{F}_{0}\right)$ of the $\mathrm{pH}$ sensitive dye, pyranine, was monitored over time. At the $\mathrm{t}=0$ seconds, the (proteo)liposomes containing buffer at $\mathrm{pH} 7.6$, were diluted 1:10 (v/v) into the same buffer at $\mathrm{pH} 6.6$, imposing a $\Delta \mathrm{pH}$ of 1 across the membrane. The buffer with a $\mathrm{pH} 6.6$ was supplemented with the substrate, taurocholate (sodium salt, $1 \mathrm{mM}$ ) (orange and magenta lines). In the case of AcrAB proteoliposomes (black line), MBX3132 $(1 \mu \mathrm{M})$ was added additionally. Fluorescence values were normalized using $\mathrm{F} / \mathrm{F}_{0}$, where $\mathrm{F}_{0}$ is the initial value at $\mathrm{t}=0$ seconds. Fluorescence was measured at $\lambda_{\mathrm{ex}}=460 \mathrm{~nm} ; \lambda_{\mathrm{em}}=510 \mathrm{~nm}$. Each point is an average of three separate measurements $(\mathrm{n}=3)$.

As shown on Figure 29 and Figure 35, control liposomes (empty liposomes) show quenching of the pyranine fluorescence, indicating that some proton leakage is taking place, due to the interaction of the compounds (taurocholate and MBX3132) with the membrane. While quenching stabilizes for the control liposomes, proteoliposomes containing AcrAB in the presence of the inhibitor MBX3132 continue to show a decrease in the fluorescence signal 
over time. The continuous decrease of fluorescence mediated by proton influx could be caused by apparent residual proton flux through AcrB in AcrAB proteoliposomes.

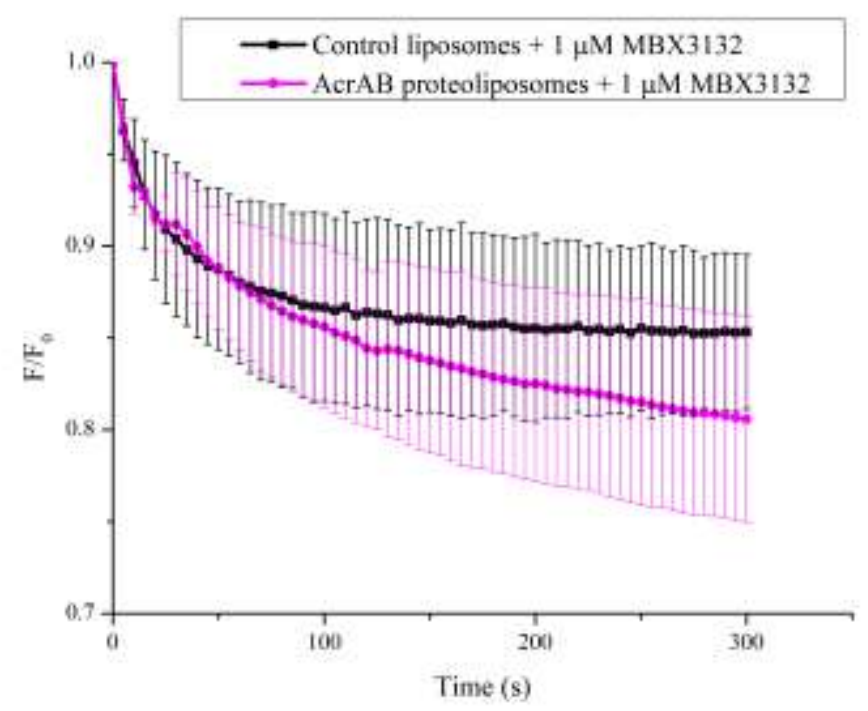

Figure 35. General influence of MBX3132 on the liposomes and protein activity. $\mathrm{H}^{+}$-transport in AcrAB proteoliposomes and non-protein containing liposomes. The change of fluorescence $\left(\mathrm{F} / \mathrm{F}_{0}\right)$ of the $\mathrm{pH}$ sensitive dye, pyranine, was monitored over time. At the $\mathrm{t}=0$ seconds, the (proteo)liposomes containing buffer at $\mathrm{pH} 7.6$, were diluted 1:10 (v/v) into the same buffer at $\mathrm{pH} 6.6$, imposing a $\Delta \mathrm{pH}$ of 1 across the membrane. The buffer with a $\mathrm{pH} 6.6$ was supplemented with the substrate, taurocholate (sodium salt, $1 \mathrm{mM}$ ) and inhibitor, MBX3132 $(1 \mu \mathrm{M})$. Fluorescence values were normalized using F/F 0 , where $\mathrm{F}_{0}$ is the initial value at $\mathrm{t}=0$ seconds. Fluorescence was measured at $\lambda_{\mathrm{ex}}=460 \mathrm{~nm} ; \lambda_{\mathrm{em}}=510 \mathrm{~nm}$. Data points are average of two consecutive measurements $(n=2)$.

Phe-Arg- $\beta$-naphthylamide (PA $\beta N$, also known by the name of MC-207,110) is the best studied inhibitor of $P$. aeruginosa RND efflux pumps (Figure 33). It has been indicated that $\mathrm{PA} \beta \mathrm{N}$ is a broad-spectrum EPI that is found to be active against several efflux pumps, like MexAB-OprM, MexCD-OprJ and MexEF-OprN (Olga Lomovskaya et al., 2001). Numerous studies were done in order to analyse the influence of this inhibitor. One study compared the influence of inhibitor from the MBX class (MBX2319) and PA $\beta N$ on AcrB activity. Higher concentrations of MBX2319 (1 to $10 \mu \mathrm{M}$ ) completely inhibited nitrocefin efflux, while PA $\beta \mathrm{N}$ at concentrations ranging from 0.2 to $10 \mu \mathrm{M}$ had no effect on the efflux of nitrocefin. These results suggested that $\mathrm{PA} \beta \mathrm{N}$ does not directly affect $\mathrm{AcrB}$ function at lower concentrations (Opperman \& Nguyen, 2015). 


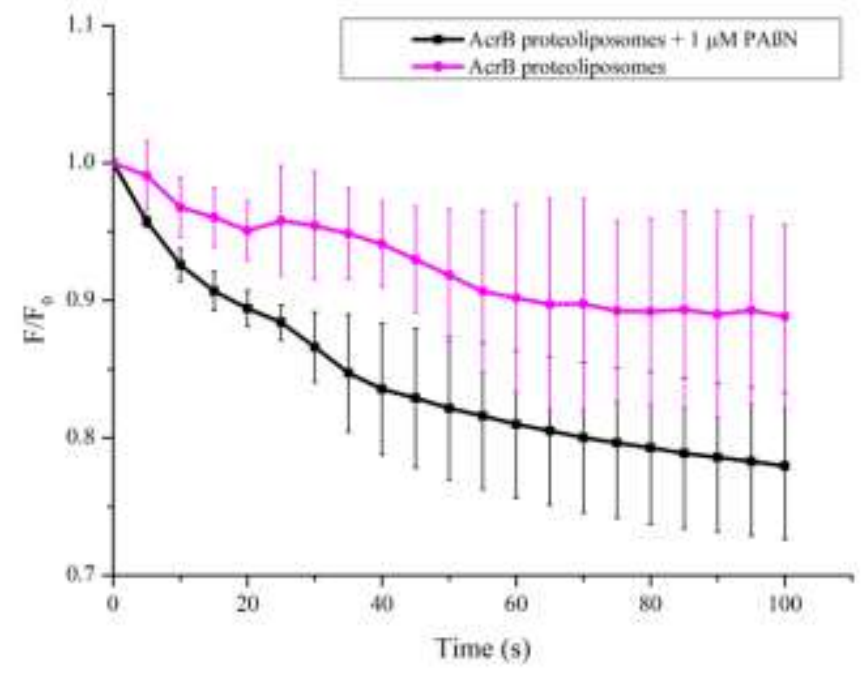

Figure 36. Effect of Phe-Arg- $\beta$-naphthylamide $(\mathrm{PA} \beta \mathrm{N})$ on $\mathrm{H}^{+}$influx into proteoliposomes containing AcrB. The change of fluorescence $\left(\mathrm{F} / \mathrm{F}_{0}\right)$ of the $\mathrm{pH}$ sensitive dye, pyranine, was monitored over time. At the $\mathrm{t}=0$ seconds, the (proteo)liposomes containing buffer at $\mathrm{pH} 7.6$, were diluted 1:10 (v/v) into the same buffer at $\mathrm{pH} 6.6$, imposing a $\Delta \mathrm{pH}$ of 1 across the membrane. The buffer with a $\mathrm{pH} 6.6$ was supplemented with the substrate, taurocholate (sodium salt, $1 \mathrm{mM}$ ) and inhibitor, $\operatorname{PA} \beta \mathrm{N}(1 \mu \mathrm{M})$. Fluorescence values were normalized using $\mathrm{F} / \mathrm{F}_{0}$, where $\mathrm{F}_{0}$ is the initial value at $\mathrm{t}=0$ seconds. Fluorescence was measured at $\lambda_{\mathrm{ex}}=460 \mathrm{~nm} ; \lambda_{\mathrm{em}}=510 \mathrm{~nm}$. Data points are average of two consecutive measurements $(\mathrm{n}=2)$.

To further investigate the behaviour of AcrB in the presence of this efflux pump inhibitor, the assay was performed where $1 \mu \mathrm{M}$ PA $\beta \mathrm{N}$ was added to proteoliposomes containing AcrB wild type, together with $1 \mathrm{mM}$ of the substrate taurocholate. The result was compared with the result of AcrB wild type proteoliposomes incubated only with taurocholate. As seen on Figure 36, a significant decrease in the fluorescence signal of AcrB proteoliposomes incubated with PA $\beta \mathrm{N}$ was observed. The decrease of the fluorescent signal intensity is even more reduced then when MBX3132 was used (Figure 34). Considerable interactions to both the groove and the cave region of deep binding pocket suggested that MBX inhibitors prevent substrates from entering deep binding pocket and/or conformationally arrest the protomer in the tight (T) state (Kobylka et al., 2020)(Sjuts et al., 2016)(Nakashima et al., 2013). However, in this study, it is observed that the presence of either increases the proton flux.

AcrB D407N, contains a substitution in the proton relay (D407, D408, K940) of AcrB. Previous analysis has shown that a titratable group on position 407 or 408 is essential for the ability to confer a drug resistance phenotype to E. coli. AcrA seems not to increase the activity of AcrB wild type. Proteoliposomes with co-reconstituted AcrA and the AcrB D407N variant do not exhibit a proton flux. This is in stark contrast to the proton influx 
measured with reconstituted AcrB and comparable to the proteoliposomes containing AcrB D407N or with liposomes containing no reconstituted protein (Figure 32).

The data presented here indicate that AcrB is active when reconstituted in liposomes. When no protein in incorporated in the bilayer, quenching of the pyranine fluorescence is not prominent and it most likely corresponds to the leakage of $\mathrm{H}^{+}$into the liposomes. AcrA seems not to have an influence on AcrB $\mathrm{H}^{+}$-transport under the conditions tested and it does not restore the activity of the inactive variant AcrB D407N. The inhibitor of AcrB, MBX3132 most likely inhibits AcrB reconstituted in the liposomes and some residual flux of protons seems to happen when MBX3132 is present with AcrAB proteoliposomes.

\subsubsection{The $\mathrm{H}^{+}$-transport activity of the MBX3132-insensitive variant, AcrB F628L}

Another mutant of AcrB, AcrB F628L, was tested in vitro using proton transport assay in proteoliposomes. This variant was characterized in vivo as insensitive and limited in its interactions with MBX3132, which was suggested to happen because of the steric clash of the L628 side chain towards the pyranopyridine core of the MBX3132 compound (Reinke T. Müller, 2019). However, the cells producing AcrB wild type are still susceptible to the inhibitor at the same concentration $(10 \mu \mathrm{M}$ MBX3132 was used for both AcrB wild type and AcrB F628L). The mutation from Phe to Leu, in the presence of the substrates shows similar activity as AcrB wild type. The protein AcrB F628L was provided with courtesy by Dr. Reinke Müller. After reconstitution of the F628L variant in liposomes, the $\mathrm{H}^{+}$influx activity was analysed in the presence of $1 \mu \mathrm{M}$ MBX3132, $1 \mathrm{mM}$ taurocholate (sodium salt), or in the presence of $1 \mathrm{mM}$ ampicillin (member of $\beta$-lactam antibiotics) as a chosen representative from its class (Figure 37).

In the presence of substrates, taurocholate and ampicillin, AcrB F628L proteoliposomes show moderate quenching of pyranine fluorescence, indicating proton translocation through AcrB (Figure 37, black and blue curves, respectively). In the presence of MBX3132 alone, AcrB F628L proteoliposomes show decrease of the fluorescence signal, which could indicate that some proton translocation is taken place (in this case, $\mathrm{t}=25$ seconds; Figure 37, magenta curve). The decrease in fluorescence seems to be similar when using both substrates and 
inhibitor. When compared with the behaviour of the AcrAB proteoliposomes in the presence of taurocholate and MBX3132 (Figure 35), the decrease of the signal is in the similar range.

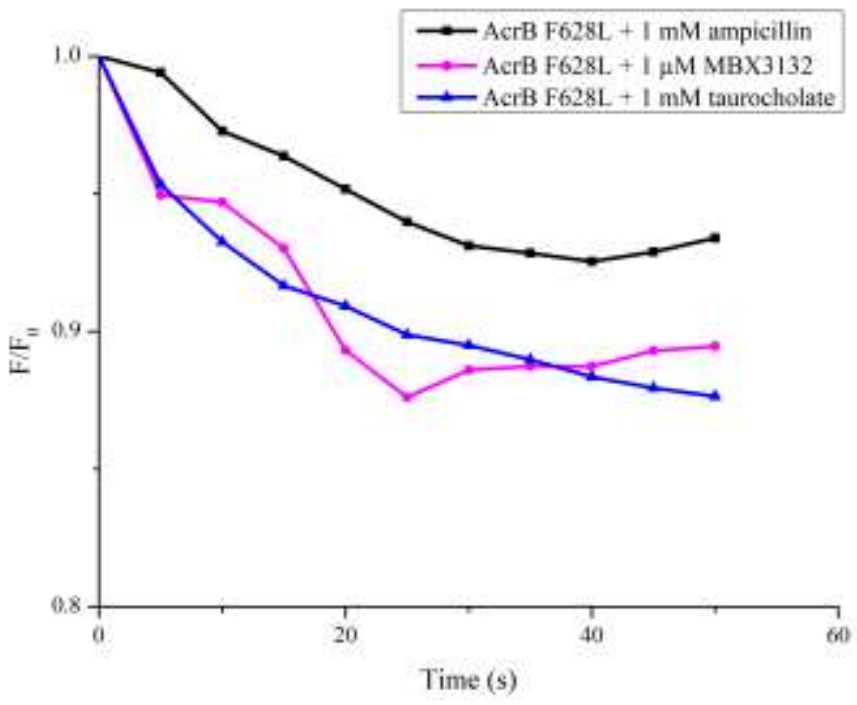

Figure 37. $\mathrm{H}^{+}$-transport in AcrB F628L proteoliposomes. The change of fluorescence $\left(\mathrm{F} / \mathrm{F}_{0}\right)$ of the $\mathrm{pH}$ sensitive dye, pyranine, was monitored over time. At the $\mathrm{t}=0$ seconds, the (proteo)liposomes containing buffer at $\mathrm{pH}$ 7.6, were diluted 1:10 ( $/ \mathrm{v}$ ) into the same buffer at $\mathrm{pH} 6.6$, imposing a $\Delta \mathrm{pH}$ of 1 across the membrane. The buffer with a $\mathrm{pH} 6.6$ was supplemented with the substrates, taurocholate (sodium salt, $1 \mathrm{mM})$, ampicillin $(1 \mathrm{mM})$ or inhibitor, MBX3132 $(1 \mu \mathrm{M})$. Fluorescence values were normalized using $\mathrm{F} / \mathrm{F}_{0}$, where $\mathrm{F}_{0}$ is the initial value at $\mathrm{t}=0$ seconds. Fluorescence was measured at $\lambda_{\mathrm{ex}}=460 \mathrm{~nm}$; $\lambda_{\mathrm{em}}=510 \mathrm{~nm}$.

\subsubsection{In vitro assembly of the efflux system AcrAB-TolC is independent of the presence of substrate}

The full assembly of the efflux pump is a complex process. Recent investigation of the pump included Cryo-ET measurements in situ that observed intermediate AcrAB complexes without TolC and discovered the importance of peptidoglycan in the periplasm for the tripartite assembly (Shi et al., 2019). According to this data, a mechanism of complex formation was proposed. AcrB and AcrA make first the bipartite complex, AcrAB. In the next step, AcrA recruits TolC and thus forms the tripartite complex already in the absence of substrate. When the substrate is present, it will be captured by the inner membrane component, AcrB, and conformational changes will occur to open TolC, so that the substrate can be expelled out of the cell (Shi et al., 2019). The structural and functional data were taken together for the modelling of the mechanism of tripartite efflux pump AcrAB-TolC first by 
Du et al in 2015. First pseudo-atomic structural data of AcrAB-TolC were obtained by Du et al in 2014, and later by Daury et al in 2016. In 2017, higher resolution structure of the pump was obtained by Wang et al (Du et al., 2014)(Du et al., 2015)(Daury et al., 2016)(Wang et $a l .$, 2017). Later, tripartite structures of the homolog MexAB-OprM were reported (Tsutsumi et al., 2019)(Glavier et al., 2020).

Several independent studies were done where it was observed whether individual components are interacting with each other. In vivo cross-linking studies on this topic showed that AcrA and TolC, as well as AcrB and TolC interact (Touzé et al., 2004). Another in vivo study using cross-linking showed that AcrA and TolC can interact in the absence of AcrB (Husain et al., 2004). Debate is still going on about AcrB and TolC interaction. Two independent studies were done on this topic, one suggests that AcrB-TolC can form a complex through a two-step mechanism via transient tip-to-tip interaction of AcrB and TolC (Tamura et al., 2005). The second study was done using computer simulations and again suggested direct and stable tipto-tip interaction between the outer membrane channel TolC and RND efflux transporter AcrB (Schmidt et al., 2016). The study from Shi et al, that was done in situ, did not observe interactions between AcrA and TolC nor between AcrB and TolC (Shi et al., 2019).

For the analysis of the $\mathrm{H}^{+} /$substrate antiport kinetics of the tripartite pump in an in vitro system, an important step is to show assembly of the full pump (Figure 22). The work presented here shows that all three components of the AcrAB-TolC pump can have full assembly in vitro, without using any cross-linking agents.

\subsubsection{MagStrep-mediated pull-down of proteoliposomes as a tool for detection of the tripartite complex formation}

The streptavidin-biotin system is a protein-ligand interaction which is one of the strongest non-covalent bonds in nature. It captures the small molecule - biotin (vitamin B7), with femtomolar affinity and this complex between streptavidin and biotin is resilient to harsh conditions (pH, temperature, denaturation) (Michael Green, 1990)(González et al., 1999). Streptavidin is a tetramer, and it can bind four molecules of biotin. The characteristics of the streptavidin-biotin complex (their interaction, affinity, resistance, etc.) are used for variety of purposes such as: biomolecule labelling, purification, immobilization and patterning 
(Howarth \& Ting, 2008). One way to use this complex is for discovering the interactions between the components of the multicomponent systems.

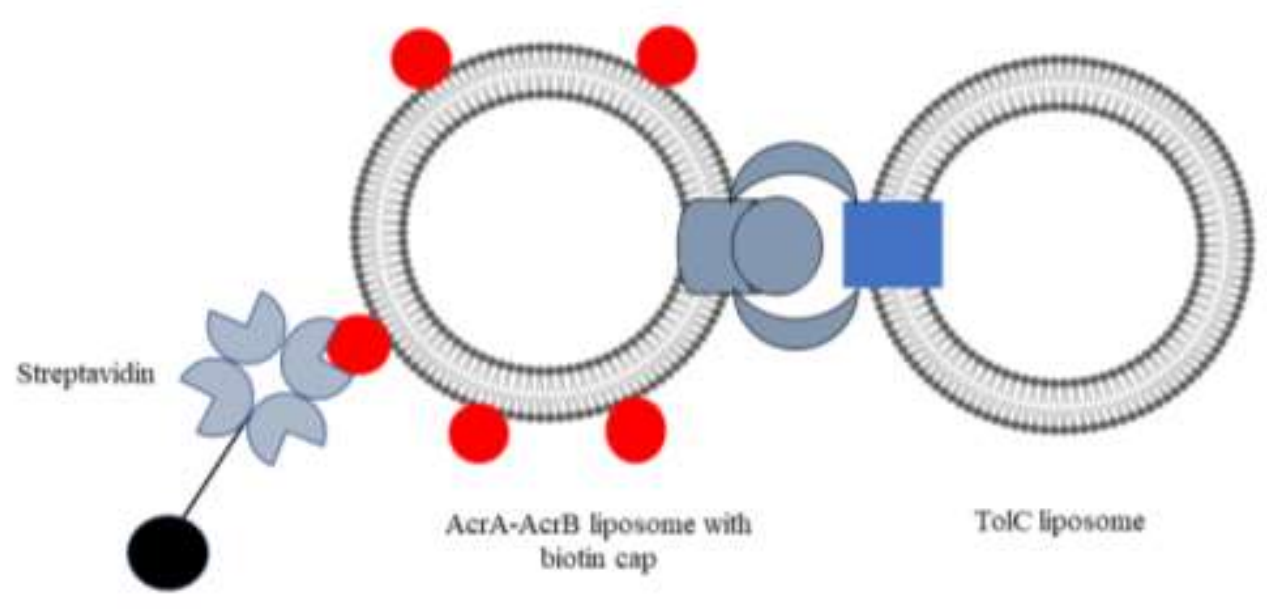

Figure 38. Streptavidin-biotin complex like a tool for proving AcrAB-TolC complex formation. Both AcrAB and TolC proteoliposomes can be labelled with biotin. In this example, only the AcrAB-containing liposomes are biotinylated. The biotinylated liposomes would be pulled down with streptavidin-coated beads. If complex formation takes place, AcrAB and TolC would interact with each other, and by affinity pull-down of the biotinylated AcrAB-containing proteoliposomes, the TolC-containing proteoliposomes will co-precipitate.

The basic idea of the usage of this system in this study is that liposomes containing AcrAAcrB or TolC would have a biotin-cap which would be recognized by streptavidin that is coated onto magnetic beads (Ntsogo Enguéné et al., 2015). When biotin from the biotinylated liposomes interacts with streptavidin from the magnetic beads, and magnetic force is applied to the beads, streptavidin-coated beads would pull down the biotin-coated liposomes (Figure 38). The same result is predicted to happen when either AcrAB proteoliposomes or TolC proteoliposomes are biotin-coated.

The kinetics of complex association and dissociation is not known, which opens up a lot of questions regarding in which conditions this assay should be performed, for example: how long liposomes should be incubated together in order to associate with each other, which conditions should be used for the stabilization of the complex, after what time the components of the pump dissociate from each other.

Previous studies that investigated in vitro assembly of tripartite systems were done on the homologue of the AcrAB-TolC pump, MexAB-OprM from P. aeruginosa (Ntsogo Enguéné et al., 2015). They have shown the formation of the complex in the conditions mimicking the 
transport as well as when no such conditions were applied to the system, for example, no $\Delta \mathrm{pH}$ applied over the membrane, usage of a soluble version of the membrane fusion protein, MexA, or usage of an inactive variant of MexB with the mutation on the D407.
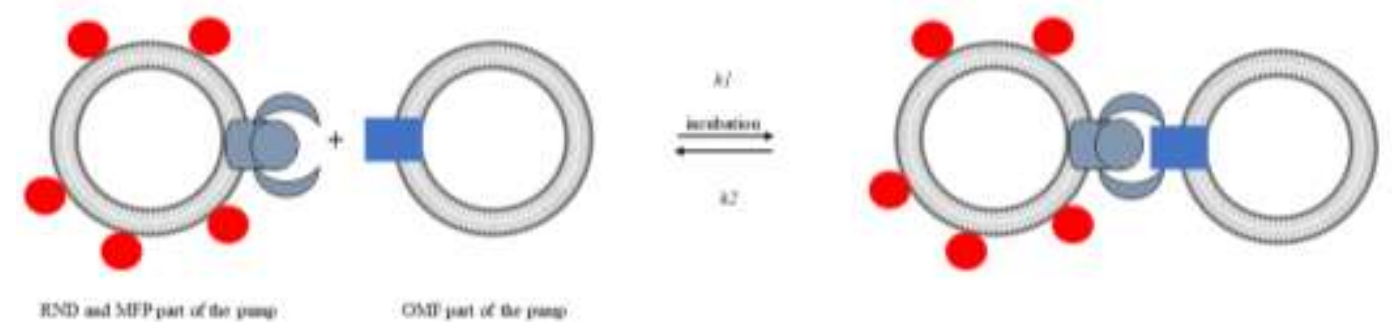

Figure 39. The pull-down assay for the observations of the association and dissociation of the components of the system. Depending on the conditions applied, it is possible to monitor the kinetic of assembly of the tripartite pump. k1/k2association/dissociation constant. The assembly/disassembly depends on the time of the incubation, as well as on the concentration of the protein components. The reversibility of the process is presented with the reversible arrows, between the non-complexed and the complexed proteoliposomes.

This pull-down assay strategy can be used for observation of the kinetics of the assembly of the pump. If shown that assembly is possible in vitro, it would be possible as well, under controlled conditions, to follow the timeline of interaction of the components of the pump. As shown in Figure 39, when proteoliposomes containing AcrA-AcrB and proteoliposomes containing TolC (either of them can be with biotin cap) are mixed, there are two possible scenarios. So far, this study was observing association of the system, as well as the dependence of the system assembly on the presence of substrate or inhibitor of the pump.

Table 14 shows the conditions tested with the first set-up of the experiment, which was to have a biotin cap on the AcrAB proteoliposomes. Conditions tested: time of the incubation of proteoliposomes (10 or 20 minutes, with or without buffer), buffer with $\Delta \mathrm{pH}=0$ or $\Delta \mathrm{pH}=1$, in the presence of the substrate $(0.2 \mathrm{mM}$ cloxacillin $)$ or inhibitor $(1 \mu \mathrm{M} / 400 \mu \mathrm{M} \mathrm{MBX} 3132)$, washing MagStrep beads or direct pelleting, amount of MagStrep beads used, testing another type of the magnetic beads (DynaBeads ${ }^{\mathrm{TM}} \mathrm{M}-280$ ).

All pulled down samples that are shown are loaded on the SDS-PAA gel directly after being precipitated and shortly incubated with SDS-PAGE sample buffer (containing $0.2 \%$ SDS).

Several conditions showed promising results regarding the complex being pulled down: 
1) when $1 \mu \mathrm{M}$ MBX3132 and proteoliposomes are incubated for 10 minutes in the buffer with $\Delta \mathrm{pH}=0$, with and without washing the MagStrep beads $(5 \mu \mathrm{l})-$ condition number 4 in Table 14 shown in Figure 40;

2) when $1 \mu \mathrm{M}$ MBX3132 and proteoliposomes are incubated for 10 minutes in the buffer with $\Delta \mathrm{pH}=0$, with washing the MagStrep beads $(10 \mu \mathrm{l})-$ condition number 9 in Table 14 shown in Figure 40;

3) when $1 \mu \mathrm{M}$ MBX3132 and proteoliposomes are incubated for 10 minutes in the buffer with $\Delta \mathrm{pH}=0$, with the Dynabeads ${ }^{\mathrm{TM}} \mathrm{M}-280$ beads $(10 \mu \mathrm{l})$ - condition number 10 in Table 14 shown in Figure 40;

4) when $1 \mu \mathrm{M}$ MBX3132 and proteoliposomes are incubated for 5 minutes in the buffer with $\Delta \mathrm{pH}=1$, with the MagStrep beads, without washing $(10 \mu \mathrm{l})$ - condition number 5 in Table 14. 
Table 14. Tested conditions for the in vitro assembly of the AcrAB-TolC system. All the conditions are tested with the AcrAB-biotin cap labelled proteoliposomes. Three out of ten different conditions (differences: beads used, buffers, washing steps, substrate/inhibitor used, etc.) showed complex formation.

\begin{tabular}{|c|c|c|c|c|c|c|c|c|c|c|}
\hline ह & & 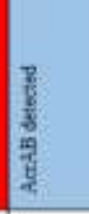 & 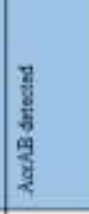 & 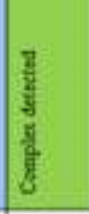 & 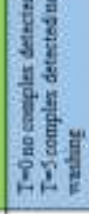 & 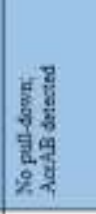 & & 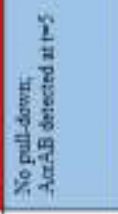 & $\begin{array}{l}\frac{7}{8} \\
\frac{8}{3} \\
\frac{8}{8} \\
\frac{8}{3} \\
\end{array}$ & 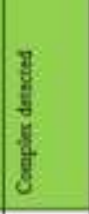 \\
\hline 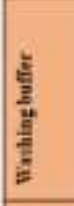 & 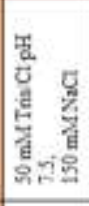 & 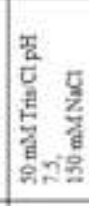 & 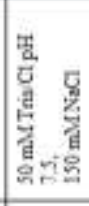 & 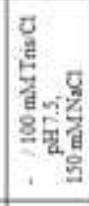 & 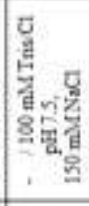 & 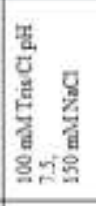 & 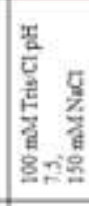 & 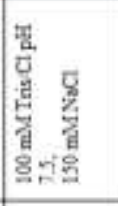 & 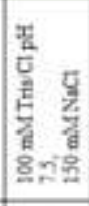 & 崫 \\
\hline 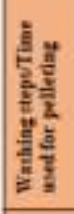 & 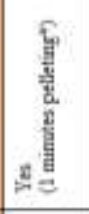 & 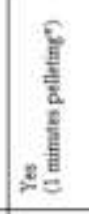 & 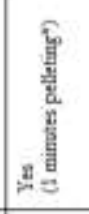 & 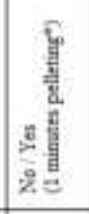 & 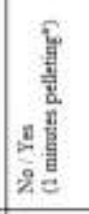 & 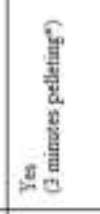 & 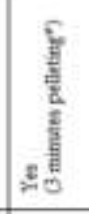 & 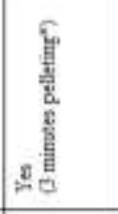 & 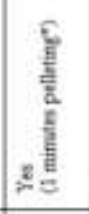 & 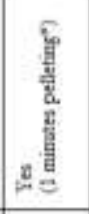 \\
\hline 童 & (. & . & 11 & 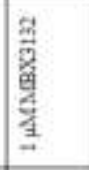 & 商 & 䞄 & 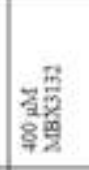 & 学 & 高 & 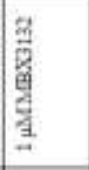 \\
\hline 蒫 & 1 & 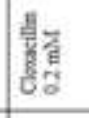 & 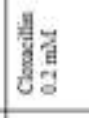 & . & . & . & . & . & . & . \\
\hline 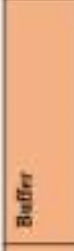 & 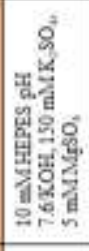 & 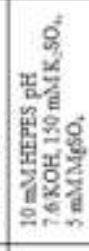 & 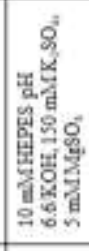 & 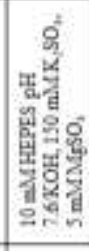 & 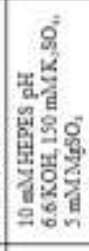 & 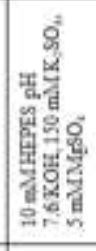 & 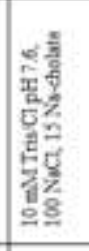 & 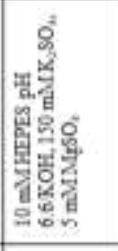 & 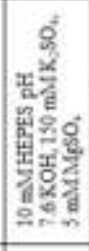 & 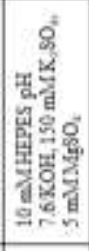 \\
\hline 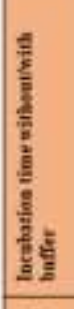 & 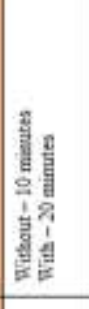 & 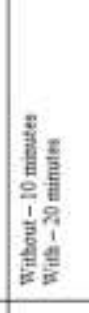 & 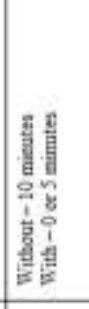 & 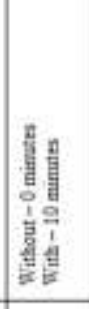 & 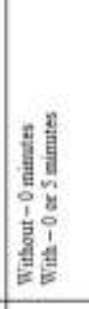 & 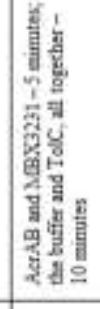 & 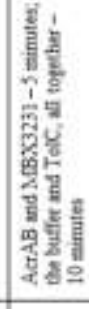 & 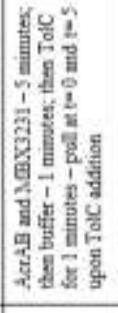 & 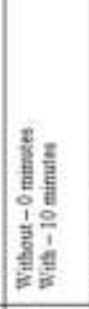 & 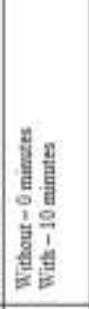 \\
\hline $\begin{array}{l}1 \\
\text { if } \\
\text { 章 } \\
\end{array}$ & a & In & $\exists$ & $\stackrel{\pi}{\Omega}$ & $a_{0}^{\pi}$ & वे & בै & वे & $\frac{\pi}{2}$ & a \\
\hline 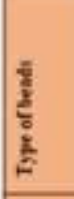 & 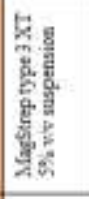 & 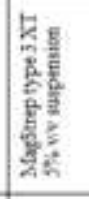 & 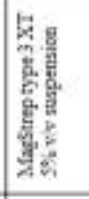 & 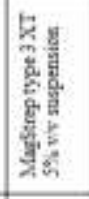 & 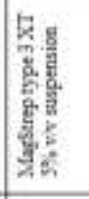 & 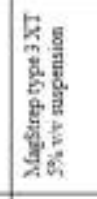 & 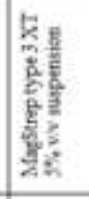 & 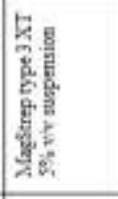 & 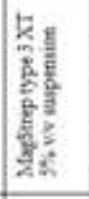 & 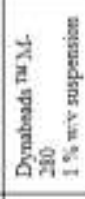 \\
\hline है & - & id & . & + & 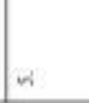 & 6 & - & - & a & 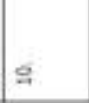 \\
\hline
\end{tabular}




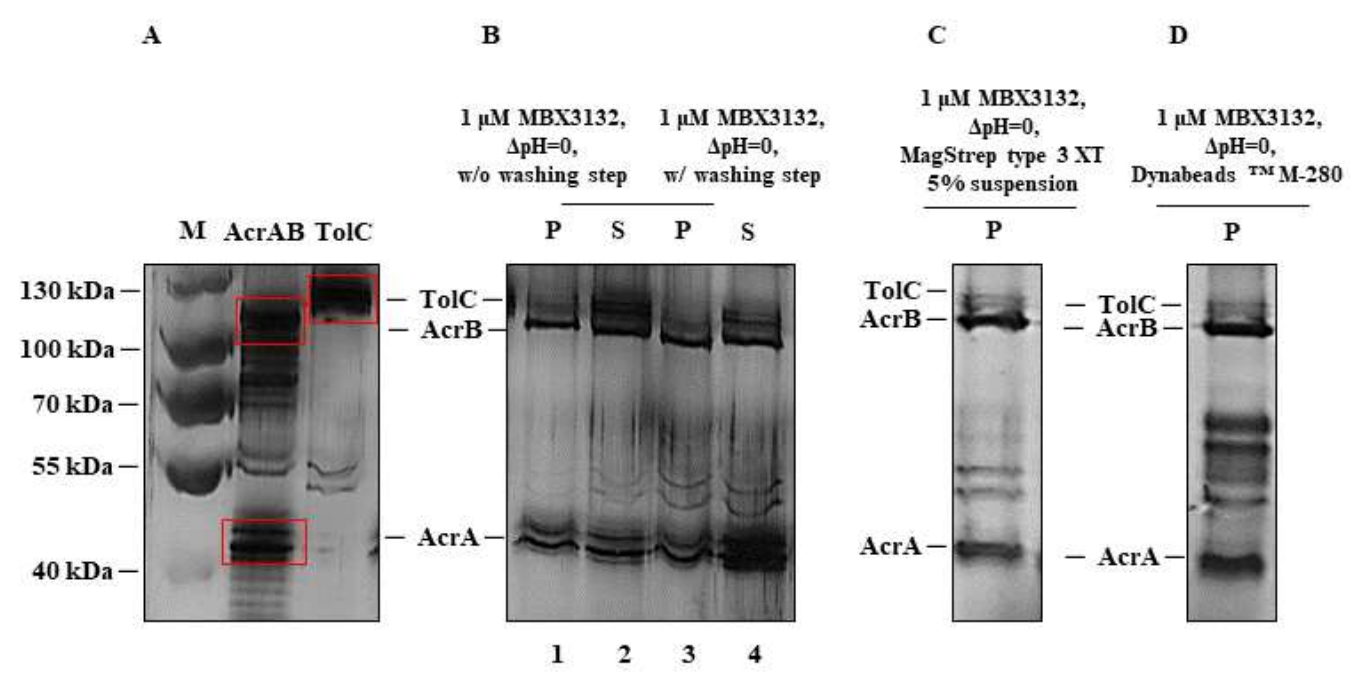

Figure 40. Pull-down experiments with AcrAB biotinylated proteoliposomes. The conditions shown in the figure correspond to the conditions described in Table 14. (conditions 4, 9 and 10, respectively). A. M-Marker (5 $\mu$ l) (PageRuler Prestained Protein Ladder (ThermoScientific)); AcrAB and TolC proteoliposome samples (15 $\mu \mathrm{l})$ before mixing the two batches of proteoliposomes. B. Samples of a pull-down $(20 \mu \mathrm{l})$ performed in the presence of $1 \mu \mathrm{M}$ MBX3132 and without $\mathrm{pH}$ gradient $(\Delta \mathrm{pH}=0)$, without $(\mathrm{w} / \mathrm{o})(1,2)$ or with $(\mathrm{w})(3,4)$ washing step. P- pull-down sample $(1,3)$; S- supernatant; proteins that are not pulled by beads $(2,4)$ (condition 4). C. Samples of a pull-down $(20 \mu l)$ in the presence of $1 \mu \mathrm{M} \mathrm{MBX3132}$ and without $\mathrm{pH}$ gradient $(\Delta \mathrm{pH}=0)$, using MagStrep type $3 \mathrm{XT}$ beads (condition 9). D. Samples of a pull-down $(20 \mu \mathrm{l})$ in the presence of $1 \mu \mathrm{M}$ MBX3132 and without $\mathrm{pH}$ gradient $(\Delta \mathrm{pH}=0)$ ), using Dynabeads ${ }^{\mathrm{TM}} \mathrm{M}-280$ beads (condition 10$)$. The samples were analysed via $10 \%$ SDS-PAA gel and silver-stained.

Even though the first experiments showed promising results, the number of conditions where the complex formation was observed were not as high as expected. Additionally, the formation of the complex was observed when the inhibitor of the pump was present, $1 \mu \mathrm{M}$ MBX3132. For investigation of the pump activity, it would be necessary to have a substrate of the pump present, rather than an inhibitor. This is especially important for the kinetic observations of the substrate transport.

In order to obtain the data about the complex formation in the presence of the substrate and/or inhibitor in more conditions, new experimental set up was used. Instead of having biotin cap on AcrAB proteoliposomes, this time the TolC proteoliposomes are biotinylated.

AcrAB proteoliposomes were mixed with TolC proteoliposomes that have a biotin cap (3.2.3.2). TolC proteoliposomes will be pulled down with streptavidin coated magnetic beads. If AcrAB proteoliposomes interact with TolC proteoliposomes, all three proteins would be detected via SDS-PAGE analysis.

Complex formation was observed independent of the presence of a $\mathrm{pH}$ gradient across the membranes. Complex formation was detected after 10 minutes of incubation of the different 
proteoliposomal batches (as indicated in the methods part, 3.2.3.2); an even more prominent interaction between proteoliposomes is detected after one hour of incubation, in absence or presence of substrate (Figure 41A). As a control, an aliquot of each batch of AcrAB or TolC proteoliposomes was subjected to SDS-PAGE analysis (Figure 40A, Figure 41A).

As shown in the study of complex assembly in situ (Shi et al., 2019), MBX3132 (inhibitor) locks the AcrB pump in a more homogenous conformation (predominantly in the symmetric TTT conformation) which helped that all the components of the system can interact and form stable complex. In this study, the tripartite assembly is observed in vitro also in the presence of the MBX3132, even in absence of a $\mathrm{pH}$ gradient.

Figure 42B shows in the line 2 (S-supernatant), how much protein is not pulled down by streptavidin; this represent the amount of the proteoliposomes that are not bound by the beads. This is also the proof that all three proteins are present in the mixture before the pulldown.

Previous studies showed very diverse results on the AcrB-TolC interaction in vitro and in vivo. This study shows that AcrB can interact with TolC even without cross-linking agent and without $\mathrm{pH}$ gradient applied, similar as seen for the complex formation with nanodiscsreconstituted AcrAB-TolC (Daury et al., 2016). For this experiment two different scenarios were used: AcrB biotinylated proteoliposomes that are mixed with TolC proteoliposomes and TolC biotinylated proteoliposomes that are mixed with AcrB proteoliposomes. In both cases, both proteins were detected on the gel, indicating that both are pulled down since the interaction between them happened. 
A

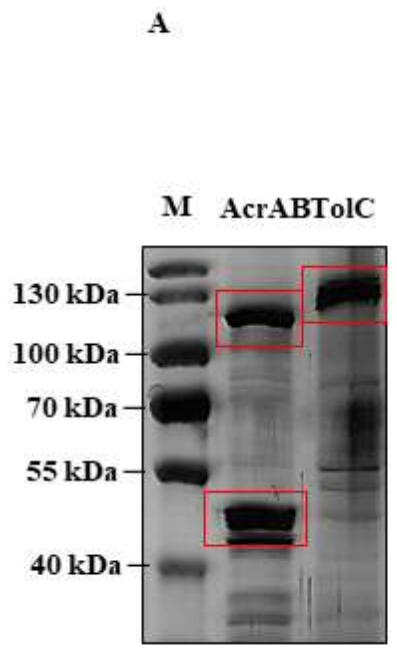

B

$1 \mathrm{mM}$ taurocholate, taurocholate, $\Delta \mathrm{pH}=1, \quad \Delta \mathrm{pH}=1$, $t=0$ minutes $t=5$ minutes P

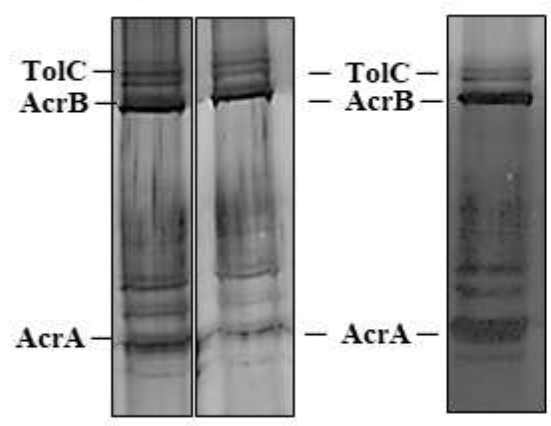

C

no substrate,
no inhibitor,

no inhibitor,

$\Delta \mathrm{pH}=0$,
ncubation 1

$\mathbf{P}$

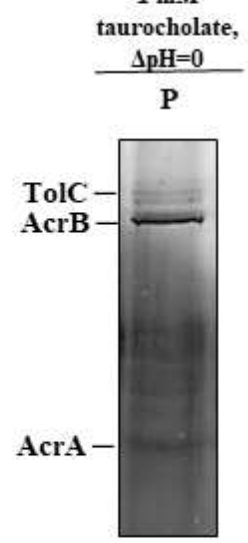

Figure 41. Formation of the tripartite system AcrAB-TolC. If the complex formation takes place, and AcrAB interacts with TolC, all three proteins would be detected on the SDS-PAA gel. Pull down experiments performed with TolC biotinylated proteoliposomes. A. M-Marker (5 $\mu \mathrm{l}$ ) (PageRuler Prestained Protein Ladder (ThermoScientific)); AcrAB and TolC proteoliposome samples before mixing and pull-down $(15 \mu \mathrm{l})$. B. Samples of a pull-down $(20 \mu \mathrm{l})$ performed in the presence of $1 \mathrm{mM}$ taurocholate and with $\mathrm{pH}$ gradient $(\Delta \mathrm{pH}=1)$, pulled at $\mathrm{t}=0$ minutes $(1)$ or $\mathrm{t}=5$ minutes $(2)$. $\mathrm{P}$ - pull-down sample $(1$, 2). C. Samples of a pull-down $(20 \mu \mathrm{l})$ in the absence of substrate and without $\mathrm{pH}$ gradient $(\Delta \mathrm{pH}=0)$, incubation time of AcrAB and TolC proteoliposomes $t=1$ hour (h). P- pull-down sample. D. Samples of a pull-down $(20 \mu \mathrm{l})$ in the presence of $1 \mathrm{mM}$ taurocholate and without $\mathrm{pH}$ gradient $(\Delta \mathrm{pH}=0)$. P-pull-down sample. The samples were analysed via $10 \%$ SDS-PAA gel and silver-stained.

Figure 42C shows that AcrB and TolC interact with each other, in the presence of substrate, $1 \mathrm{mM}$ taurocholate, in the case where the AcrB proteoliposomes are biotinylated and are pulled down, as well as when TolC containing proteoliposomes are biotinylated and pulled down. This goes with the assumption that when AcrB and TolC present in either proteoliposomes are in close proximity and have possibility to make tip-to-tip interaction, it is possible that they form a complex. It has to be mentioned that there is a possibility, that in all the experiments, some unspecific co-precipitation took place. 
A

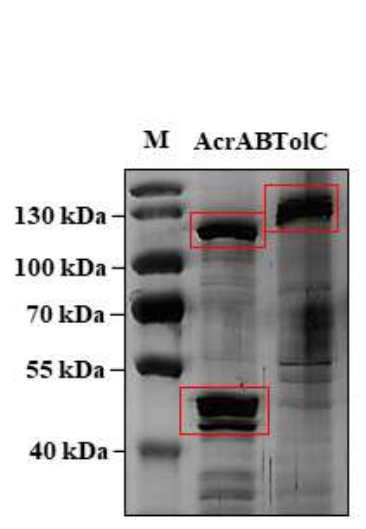

B

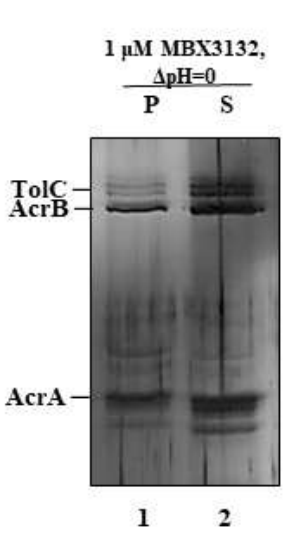

C

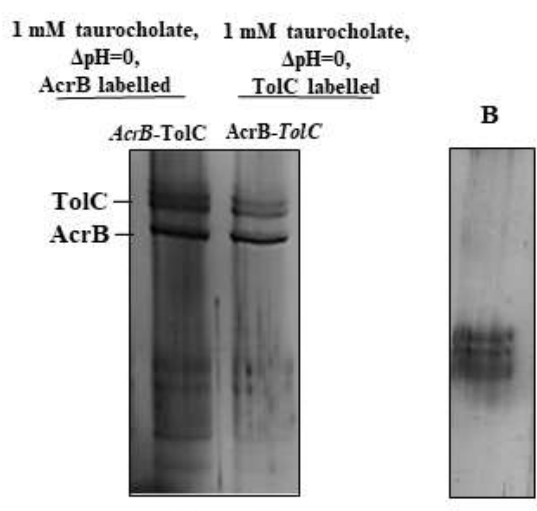

Figure 42. Formation of the tripartite system AcrAB-TolC. If the complex formation takes place, and AcrAB interacts with TolC, all three proteins would be detected on the SDS-PAA gel. Pull-down experiments performed with TolC biotinylated proteoliposomes. A. M-Marker $(5 \mu \mathrm{l})$ (PageRuler Prestained Protein Ladder (ThermoScientific)); AcrAB and TolC proteoliposome samples $(15 \mu \mathrm{l})$. B. Samples of a pull-down $(20 \mu \mathrm{l})$ performed in the presence of $1 \mu \mathrm{M}$ MBX3132 and without $\mathrm{pH}$ gradient $(\Delta \mathrm{pH}=0)$. $\mathrm{P}$ - pull-down sample (1). S-supernatant; proteins that are not pulled down by beads (2). C. Samples of a pull-down $(20 \mu \mathrm{l})$ in the presence of $1 \mathrm{mM}$ taurocholate and without $\mathrm{pH}$ gradient $(\Delta \mathrm{pH}=0)$. AcrB biotinylated proteoliposomes were pulled with TolC proteoliposomes (3) and TolC biotinylated proteoliposomes were pulled with AcrB proteoliposomes (4). D. Sample of MagStrep beads only (B). The samples were analysed via 10\% SDS-PAA gel and silver-stained.

\subsubsection{Derivation of reconstitution conditions via $\mathbf{R}_{\text {sat }}$ and $\mathbf{R}_{\text {sol }}$}

The methodology used for the preparation of AcrB (proteo)liposomes for the in vitro activity assay is detergent-mediated reconstitution. The reconstitution starts with membrane solubilization which is divided into three stages. During stage I, detergent present in the sample is in equilibrium between single molecules and detergent micelles. In the presence of liposomes, detergent is incorporated into their membranes. The second stage represents the point where detergent-saturated liposomes are in equilibrium with detergent-phospholipid mixed micelles. At the last stage, only a mixture of detergent-phospholipid micelles is present. The different stages and their transitions are detected by the light scattering of the liposome solution (Figure 18, 1.7.4). Figure 43 shows the membrane solubilization process in the case of liposomes prepared for the reconstitution of AcrB, AcrAB, AcrB D407N, AcrAB D407N and empty liposomes, for the assay for monitoring the activity of the AcrAB pump. 

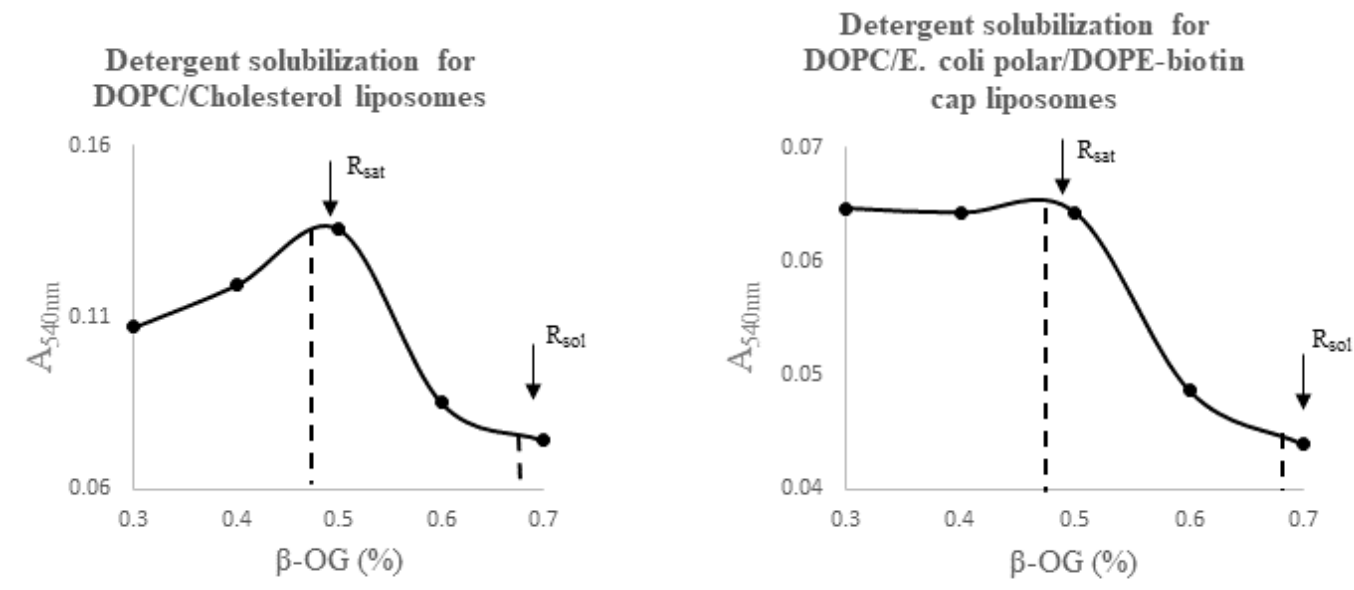

Figure 43. $\mathrm{R}_{\text {sat }} / \mathrm{R}_{\text {sol }}$ determination. The graphs show the obtained results for onset and total solubilization for the liposomes used in the AcrB functional assay (1,2-Dioleoyl-sn-glycero-3-phosphocholine (DOPC)/Cholesterol, ratio 3:1, w/w) and in vitro assembly assay (DOPC/Cholesterol with addition of 1,2-dioleoyl-sn-glycero-3-phosphoethanolamine (DOPE), end ratio 30:10:1, w/w/w). The absorbance of the lipid/detergent mixture was measured at $540 \mathrm{~nm}$ at different concentrations of the detergent, $\beta$-OG.

In short, the first stage is followed with a slight increase of the signal, until the $\mathrm{R}_{\text {sat }}$ point; at the $\mathrm{R}_{\mathrm{sat}}$ point (until $\mathrm{R}_{\mathrm{sol}}$ ), turbidity of the solution decreases since the sample at this point contains the mixture of solubilized liposomes and liposomes containing detergent micelles. Total solubilization $\left(\mathrm{R}_{\mathrm{sol}}\right)$ of the vesicles is shown in the third section of the graph (after $\mathbf{R}_{\mathrm{sol}}$ point) where the turbidity is low and steady.

Performing experiments which will provide information about the amount of detergent to be added to the lipid solution is crucial. Incorporation of the protein in the artificial environment is dependent on the stability and state of it. The basic understanding of the lipid-detergent interactions is a highly significant prerequisite for efficiently seeking the optimal conditions for solubilization and reconstitution of any membrane protein (Lambert et al., 1998). $\mathrm{R}_{\text {sat }}$ and $\mathrm{R}_{\text {sol }}$ indicate the concentration range of detergent to be added to the liposomes (dependent on the lipid type and detergent) for efficient incorporation of proteins. The orientation of the protein can be influenced by the detergent to lipid ratio. The unidirectional insertion of transmembrane proteins is dependent on both the type and the concentration of detergent present in the lipid bilayer. The concentration of detergent should be close to $R_{\text {sat }}$ (just below the onset of liposome solubilization). In that case, proteins are inserted in the membrane through their most hydrophobic domains first (Lambert et al., 1998)(Rigaud \& Lévy, 2003). For instance, reconstitution at $\mathrm{R}_{\text {sat }}$ allows for unidirectional reconstitution as shown by Dezi 
et al in 2013, where they unidirectionally reconstituted three types of transmembrane proteins in GUVs (Dezi et al., 2013).

\subsubsection{Solubilization assay to validate the reconstitution of membrane proteins into artificial membranes}

In order to examine how much protein is actually incorporated in the bilayer of the liposomes during reconstitution, an appropriate test should be used. For that reason, a solubilization assay was conducted. The assay was done as described in 3.2.2.19. Figure 44 shows the SDSPAGE analysis with subsequent Coomassie-staining of the solubilized samples obtained.

Samples of proteoliposomes (3.2.2.17) were collected and prepared for according to the 3.2.2.19. Proteoliposomes were solubilized with $1 \%$ DDM before the ultracentrifugation step was performed. Samples before ultracentrifugation should contain proteins that are incorporated into the membrane of the liposomes as well as the protein that was not successfully incorporated in the liposomes (aggregates). Supernatant after ultracentrifugation should contain only the solubilized proteins that were incorporated inside the membrane, since the aggregates are not solubilized by the detergent and are pelleted during ultracentrifugation. 


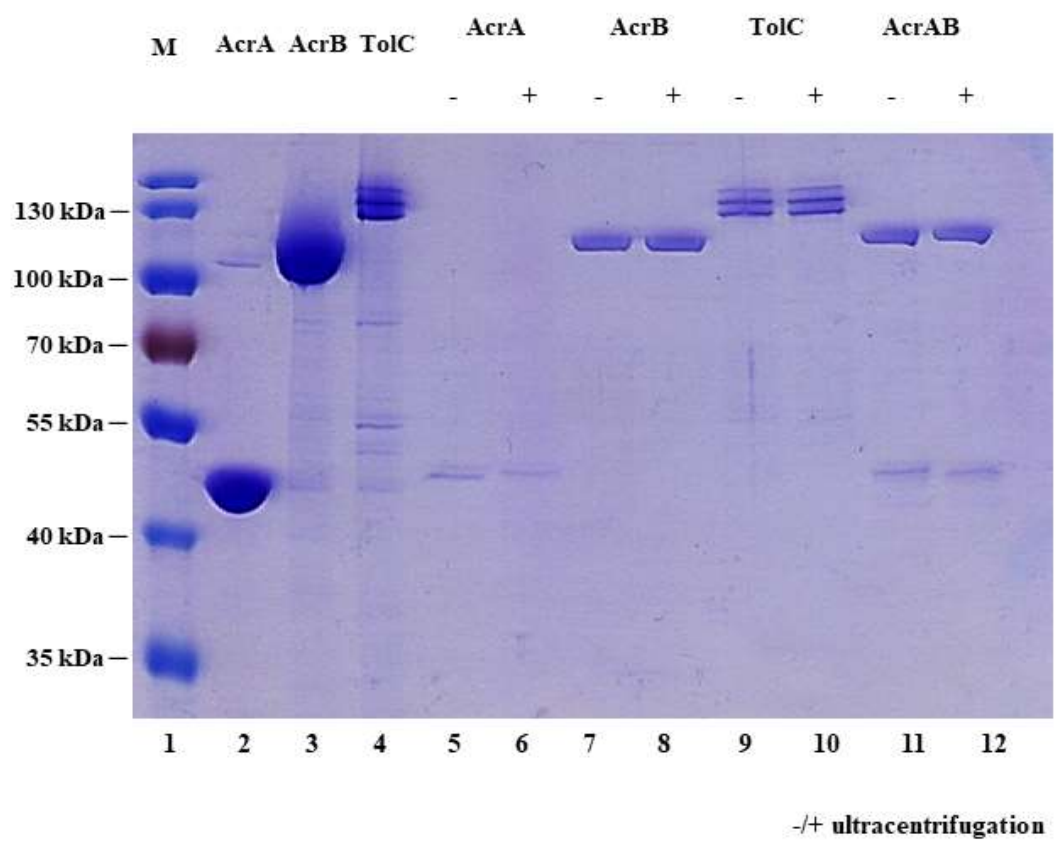

Figure 44. Solubilization assay of AcrA, AcrB, TolC and AcrAB proteoliposomes. Coomassie-stained $10 \%$ SDS-PAA gel of samples obtained during the solubilization assay. M-Marker (PageRuler Prestained Protein Ladder (ThermoScientific)) $(5 \mu \mathrm{l}, 1)$. For the calculation of the protein amount in liposomes, the sample of the purified protein was loaded on the SDSPAA gel, AcrA- $1.9 \mu \mathrm{g}(2)$, AcrB- $3.9 \mu \mathrm{g}$ (3), TolC- $1.7 \mu \mathrm{g}$ (4). AcrA proteoliposomes $(5,6)$, AcrB proteoliposomes $(7,8)$, TolC proteoliposomes $(9,10)$ and AcrAB proteoliposomes $(11,12)$ are loaded $(15 \mu \mathrm{l})$ before $(-)$ and after $(+)$ solubilization and ultracentrifugation, respectively.

Table 15. Determination of the efficiency of protein incorporation in the liposome. The efficiency was defined as the percentage of the protein correctly incorporated in the liposomes (after ultracentrifugation) compared to both correctly and not correctly incorporated protein amount (before ultracentrifugation). The protein amounts were evaluated according to the Image J (Schneider et al., 2012) estimations of the band intensities before and after ultracentrifugation as shown in the Figure 44.

\begin{tabular}{|l|l|}
\hline Sample name & $\begin{array}{l}\text { Estimation of correctly incorporated protein in } \\
\text { liposomes }(\%)\end{array}$ \\
\hline AcrA in AcrA proteoliposomes & 48.51 \\
\hline AcrB in AcrB proteoliposomes & 95.39 \\
\hline TolC in TolC proteoliposomes & 90.40 \\
\hline AcrA in AcrAB proteoliposomes & 92.27 \\
\hline AcrB in AcrAB proteoliposomes & 90.41 \\
\hline
\end{tabular}

In an ideal case, bands before and after ultracentrifugation should be the same in intensity (100\% successful incorporation of the protein). For comparison of the band intensities program ImageJ was used (Schneider et al., 2012). Table 15 shows the efficiencies of the incorporation of proteins in the samples from AcrA, AcrB, AcrAB and TolC reconstitutions, 
shown in Figure 44. It has to be noted that these efficiencies can vary from batch to batch and that can also influence the quality of the samples used in the transport assays.

\subsubsection{Reconstitution efficiency and estimations of protein amount incorporated in the liposomes}

If desired, a solubilization assay can be used for estimations of the protein amount in liposomes and of the reconstitution efficiency. If, on the same gel, samples of proteoliposomes as well as samples of the same purified protein is loaded (Figure 44), the amount of protein inserted in the liposomes can be determined. The exact amount of pure protein loaded on the gel has to be known. The intensities of the bands on the Coomassiestained SDS-PAA gel can be compared. At the same time, if it is known how much protein is incorporated in the liposomes, that can be used for the estimation of the reconstitution efficiency. In that case, the amount of protein used in the reconstitution (protein amount added to the solubilized liposomes) is compared with the amount of protein that is estimated that is present in the liposomes (after Bio Beads removal, washing steps, solubilization and ultracentrifugation).

Table 16. Determination of the reconstitution efficiencies and the protein amounts incorporated in the proteoliposomes. The reconstitution efficiency was defined as the percentage of the protein correctly incorporated in the liposomes (after solubilization and ultracentrifugation) compared to protein amount used in the reconstitution (the amount of protein added to the solubilized liposomes). The protein amounts were evaluated according to the Image J (Schneider et al., 2012) estimations of the band intensities as shown in the Figure 44. The amount of protein in $100 \mu$, which is used in the AcrB activity assay in proteoliposomes, is shown.

\begin{tabular}{|l|l|l|}
\hline Sample name & Reconstitution efficiency $(\boldsymbol{\%})$ & $\begin{array}{l}\text { Protein amount used in } \\
\text { the activity assay }(\boldsymbol{\mu g})\end{array}$ \\
\hline AcrA in AcrA proteoliposomes & $8 \%$ & 0.32 \\
\hline AcrB in AcrB proteoliposomes & $98 \%$ & 4.88 \\
\hline TolC in TolC proteoliposomes & $54 \%$ & 5.42 \\
\hline AcrA in AcrAB proteoliposomes & $4 \%$ & 0.56 \\
\hline AcrB in AcrAB proteoliposomes & $83 \%$ & 4.15 \\
\hline
\end{tabular}




\subsubsection{TolC - the outer membrane protein of the AcrAB-TolC system}

TolC is an outer membrane protein from E. coli which is necessary for the extrusion of virulence factors and toxic compounds (1.6.1) (Benz et al., 1993).

The following experiment indicates the behaviour of TolC when reconstituted in liposomes and at the same time gives an indication of the conformational state of the protein (open or closed). TolC wild type is closed, hence pyranine incorporated in TolC proteoliposome will not be transported out. For the control of the experiment, empty liposomes (non-protein containing liposomes) and liposomes containing open variant of TolC, YFRS, were used.

For the TolC pyranine wash assay was performed as described in 3.2.2.20. In short, proteoliposomes and control liposomes were diluted in reconstruction buffer $(20 \mathrm{mM}$ Tris/Cl, $150 \mathrm{mM} \mathrm{NaCl}, \mathrm{pH} 7.5)$, in ratio 1:10, v/v. The samples were then incubated on ice for at least 15 minutes. Afterwards, samples were applied onto the filter membrane $(0.22 \mu \mathrm{m})$ previously washed with reconstitution buffer. Filter was washed with the same buffer, two times and then incubated for 10 minutes in PBS buffer supplemented with $1 \%$ SDS, with constant shaking at room temperature. The samples were was measured at excitation $-460 \mathrm{~nm}$ and emission $-510 \mathrm{~nm}$.

TolC wild type and TolC YFRS variant were incorporated into pyranine-loaded liposomes. Empty liposomes (without reconstituted protein) were used as a negative control. Since TolC wild type is natively closed, expectance is that after incubation, measured pyranine fluorescence is higher than the fluorescence measured in the TolC YFRS samples, since YFRS is an "open variant" of TolC. As shown in Figure 45, pyranine fluorescence signal is lower for the proteoliposomes containing TolC variant, YFRS in comparison to both TolC wild type containing- and protein-free (proteo)liposomes. This suggests that pyranine can exit through TolC YFRS, but not through TolC wild type. The empty liposome control in addition shows that pyranine does not diffuse through the membrane. TolC wild type and TolC YFRS used for this experiment were provided with courtesy by Dr. Alina Ornik-Cha. 


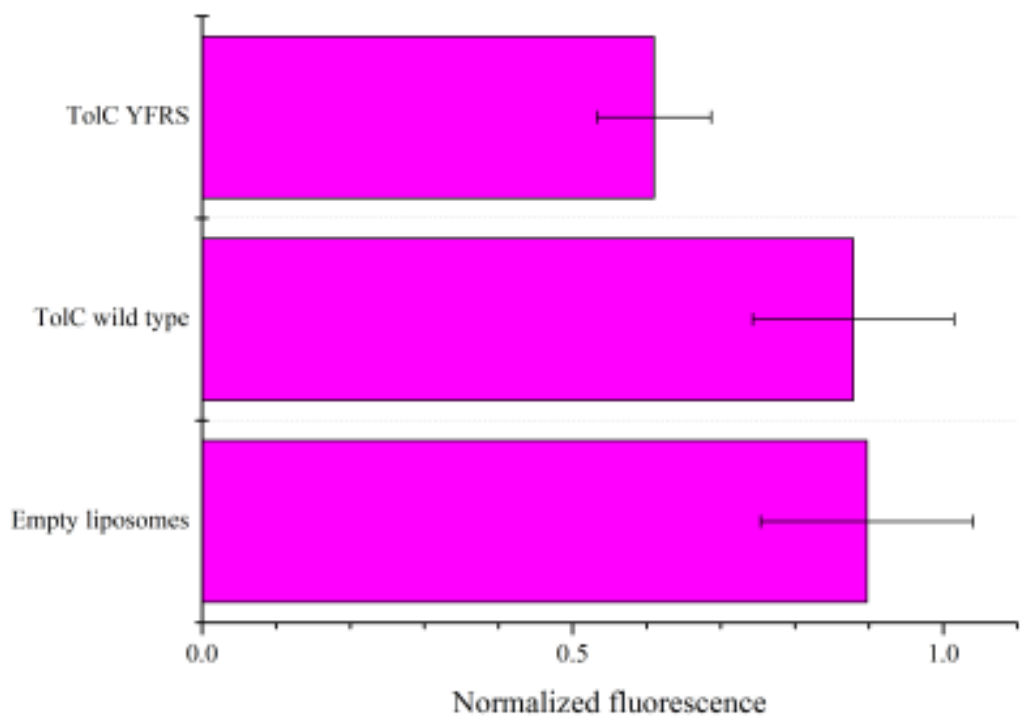

Figure 45. TolC proteoliposome pyranine efflux assay. TolC wild type is in a closed state while the TolC variant, YFRS, is in an open state. (Proteo)liposomes were diluted in reconstruction buffer $(20 \mathrm{mM}$ Tris/ $\mathrm{Cl}, 150 \mathrm{mM} \mathrm{NaCl}, \mathrm{pH} 7.5)$, in ratio $1: 10$, and incubated on ice for at least 15 minutes. Samples were applied onto the filter membrane $(0.22 \mu \mathrm{m}$, washed with reconstitution buffer). The filter was washed with the same buffer, two times and then incubated for 10 minutes in PBS buffer supplemented with $1 \%$ SDS, with constant shaking at room temperature. The TolC variant YFRS shows lower fluorescence signal compared to the wildtype TolC containing proteoliposomes and the liposomes without reconstituted protein. The experiment indicates that pyranine is diffusing out of the TolC YFRS proteoliposomes. Fluorescence values were normalized using $\mathrm{F} / \mathrm{F}_{0}$, where $\mathrm{F}_{0}$ is the initial value at $\mathrm{t}=0$ minutes. Fluorescence was measured at $\lambda_{\mathrm{ex}}=460 \mathrm{~nm} ; \lambda_{\mathrm{em}}=$ $510 \mathrm{~nm}$. Each bar represent normalization of six independent measurements $(\mathrm{n}=6)$.

In order to show the significance of the data, Student's t-test was performed (Table 17). The t- test tells how significant the differences (means) between two groups are; it tells if those differences could have happened by chance. The data of the samples are brought to the number, the t- value.

Table 17. Student's t-test analysis on TolC proteoliposomes assay.

\section{Sample comparison}

TolC wild type/Empty liposomes

TolC YFRS/Empty liposomes

TolC wild type/TolC YFRS

\section{Statistical significance}

p>.05

$\mathrm{p}<.05$

$\mathrm{p}<.05$

The results show that the p- value for the samples TolC wild type and TolC YFRS is less than the alpha value, $\mathrm{p}<.05$, (alpha is the probability of rejecting the null hypothesis when it is 
true; in this case, a $5 \%$ risk of concluding that a difference exists when there is no actual difference). This would indicate that wild type and YFRS samples do not belong to the same category of the samples, but are belonging to the different groups. That would mean that the difference between fluorescence signals of wild type and the open mutant YFRS is statistically significant. The same is true for the samples TolC YFRS and empty liposomes. However, the p- value for wild type and empty liposomes is higher than the alpha value, meaning that between these two samples the difference is not statistically significant.

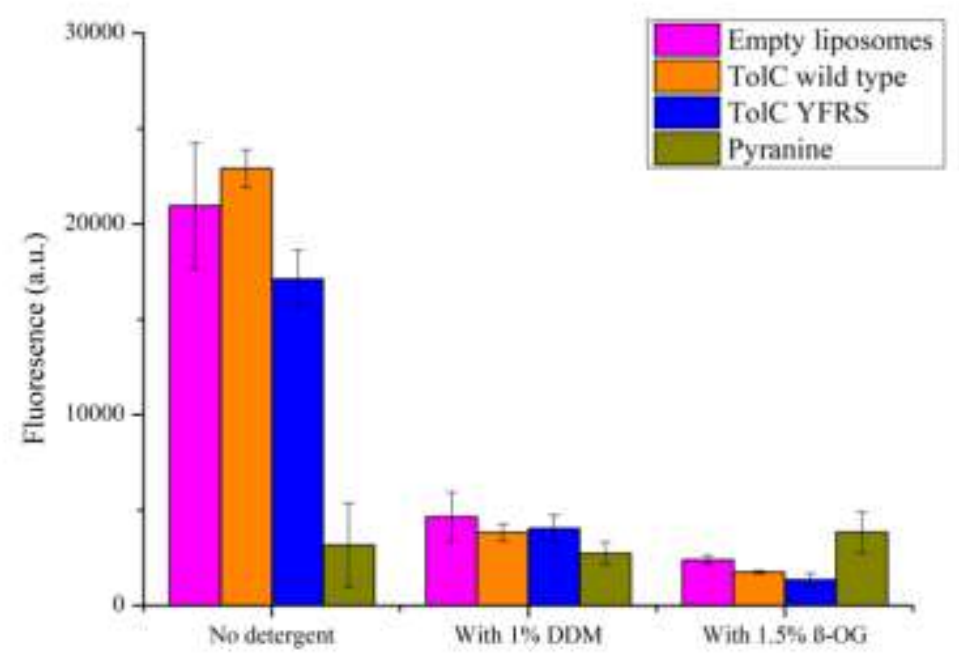

Figure 46. Complete solubilization of liposomes leads to loss of fluorescent signal. TolC wild type as well as TolC variant, YFRS, were reconstituted and proteoliposomes were subjected to the solubilization with detergents DDM and $\beta-O G$ at the concentrations indicated. As control, empty liposomes were used. The pyranine sample is the application of the same amount of pyranine as in the (proteo)liposome assay in buffer to indicate the binding of pyranine to the filter during the filter washing assay. Fluorescence was measured at $\lambda_{\mathrm{ex}}=460 \mathrm{~nm} ; \lambda_{\mathrm{em}}=510 \mathrm{~nm}$. Each bar represents average of three consecutive measurements $(n=3)$.

Another way to show that reconstitution was successful, besides the solubilization assay described in case of the AcrB proteoliposomes, as well as to show that (proteo)liposome formation happened together with encapsulation of the dye, is to simply solubilize liposomes and then measure the fluorescence signal. Here, two different detergents were used, DDM as well as $\beta$-OG, both tested for TolC purification. The percentage of the detergent used also corresponds to the percentage used for solubilization of membrane vesicles containing TolC, during purification. 
DDM was not as efficient as $\beta-\mathrm{OG}$, more residual fluorescence was measured, but the loss of the signal intensity is significant when compared with the proteoliposome samples or with control liposomes without detergent treatment (Figure 46). This indicates that proteoliposome formation took place, where pyranine was incorporated inside the proteoliposomes during the reconstitution process. When the (proteo)liposomes were solubilized and the filter washed subsequently, loss of the fluorescence is prominent - indicating efficient solubilization of the (proteo)liposomes and removal of the dye via the filter wash procedure.

\subsection{Discussion and conclusion}

\subsubsection{Pyranine as a reporter system}

For the purpose of monitoring the $\mathrm{H}^{+}$-transport activity of the efflux pump $\mathrm{AcrB}$, an in vitro assay was established where pyranine fluorescence was monitored as a function of time. As shown in Figure 24, if the $\mathrm{pH}$ of the solution rises, getting to more alkaline solution, the fluorescence rises; moreover, if we look at the AcrB proteoliposome assay set up, if the activity of the protein is present, and the protein is in right-side out orientation, the acidification of the lumen of the proteoliposome takes place (Figure 23), which leads to the quenching of pyranine fluorescence. The changes of $\mathrm{pH}$ that are happening in this assay are set up to be between $\mathrm{pH} 6$ and $\mathrm{pH}$ 8. There is a linear dependence between the fluorescence of pyranine and $\mathrm{pH}$ (Figure 24), that gives the possibility to observe the events in the lumen of the proteoliposome and to draw conclusions of the activity of the protein used. Taking into consideration this linear dependence from Figure 24 ( $\Delta \%$ fluorescence $\mathrm{F}=99.157 \mathrm{x} \Delta \mathrm{pH}$ 525.56), it can be calculated that the $\mathrm{pH}$ change in the AcrB proteoliposomes is about 0.7 while for control liposomes, $\mathrm{pH}$ of the lumen of the liposomes has changed for 0.2 (Figure 29).

The dye can be easily incorporated into the (proteo) liposome and the removal of pyranine at the outside of the proteoliposomes can be done either by washing the proteoliposomes or by passing them over the gel filtration column, or both. Another important feature is that pyranine does not diffuse through the membrane. 
With the characteristic that were summed up in 4.2 .1 , it can be concluded that pyranine is a great choice for robust, reproducible and accurate detector/reporter for the proton transport (translocation) experiment.

\subsubsection{Designing an in vitro assay for monitoring $\mathrm{H}^{+}$-transport of AcrB in an artificial membrane}

In order to establish an in vitro assay for detection of the activity of AcrB pump when reconstituted in liposomes, several types of liposomes were prepared: control liposomes (no protein incorporated), AcrB proteoliposomes, and AcrAB proteoliposomes. In order to confirm the results that are obtained, proteoliposomes with AcrB variant, D407N were prepared, as well as AcrAB D407N. Preparation of these liposomes gave an opportunity for investigating the influence of $\Delta \mathrm{pH}$ on the activity of the RND pump, when it was induced by applying $\Delta \mathrm{pH}$ over the bilayer. The influence of membrane fusion protein, AcrA, seems to be not observed in the experiments done in this study. When another variant of AcrB was used, AcrB F628L (Figure 37), the quenching of pyranine fluorescence was observed in the presence of substrates taurocholate and ampicillin, indicating proton influx. In the presence of MBX3132 the proton influx is more prominent than in the presence of ampicillin. It was suggested that because of the steric clash of the L628 side chain towards the pyranopyridine core of the MBX3132 compound, F628L is less susceptible towards this inhibitor (Reinke T. Müller, 2019).

As explained in this chapter, the $\mathrm{pH}$ sensitive dye - pyranine, was used as a reporter, and its fluorescence was monitored as a function of time. Liposomes were diluted in the buffer with acidic $\mathrm{pH}$, which created a proton gradient over the membrane. The substrate was added in the outside buffer (buffer with lower $\mathrm{pH}$ ), which triggered the activity of proteins in the liposomes in the right-side out orientation (i.e., periplasmic part of AcrB is located outside the proteoliposome) to be active (Figure 23).

Since liposomes were solubilized at $\mathrm{R}_{\text {sat }}$ for the subsequent protein incorporation, the orientation of the protein tends to be unidirectional, during which the hydrophobic parts of the protein insert first in the detergent-saturated liposomes. The lactose transport protein (LacS) of Streptococcus thermophilus has a polytopic membrane (hydrophobic carrier) 
domain and a carboxyl-terminal cytoplasmic (180 amino acids big hydrophilic) domain (B. Poolman et al., 1989); it has been shown that hydrophobic part of the protein LacS inserts first into the lipid bilayer by fusion of detergent-saturated liposomes and protein/detergent micelles (Knol et al., 1996). This would also favour reconstitution of right-side out proteoliposomes, in the case of AcrB. And only those proteins would be triggered for activity. In order to determinate the orientation of AcrB in liposomes, several experiments can be conducted: introduction of a probe via cysteine crosslinking (requires an introduction of a cysteine in a periplasmatic domain) which can be detected via fluorescence spectroscopy (for fluorescence probe) or via Electron Spin Resonance (requires spin probes). The labelling should happen after reconstitution to only label proteins in specific orientation. Additionally, electron microscopy can be used for visualization of the proteoliposomes and the protein orientation in the liposomes.

How much activity is observed during the measurement, depends on reconstitution success and efficient incorporation of the protein in the artificial membrane. These characteristics are the one that determent the quality of the sample and leads to the batch-to-batch difference when showing the intensity of the activity of AcrB and/or AcrAB.

General and reproducible observations are: AcrB reconstituted in the artificial lipid membrane is active (shows basal activity) and AcrA seems not to enhance the activity of AcrB when co-reconstituted in the liposomes (4.2.3).

It has been shown by Atzori et al in 2020, that the deep binding pocket of AcrB can be divided into sub-parts, like groove, cave, interface (Atzori et al., 2020). The indication exists that substrates of AcrB with higher molecular weight, like taurocholate, were predicted to be groove binders. For MBX3132 (Figure 33) has been shown that it tightly binds to basically the entire deep binding pocket (Sjuts et al., 2016). In addition to this, computational studies suggest the binding affinities of the AcrB inhibitor to be much higher than for AcrB substrates (Vargiu et al., 2014)(Sjuts et al., 2016). These information are considered crucial for interpreting the data gathered in this study. The assay in this work observes the proton translocation, not the substrate translocation. When both substrate and inhibitor are present in the solution with AcrB or AcrAB proteoliposomes, quenching of pyranine fluorescence has been observed indicating proton translocation. Possible explanation could be that the inhibitor causes a "leakage" in AcrB, a shortcut, and the protons are then being transported in an uncoupled fashion through AcrB. The inhibitor would bind to AcrB (higher affinity) and 
prevent the activity. However, due to possible shortcut, the inhibitor can mimic a state in which the transmembrane domain is permeable for protons. Against this interpretation is the observation that the $\mathrm{MBX}$ inhibitor does not compromise the proton motive force in $E$. coli to an extent that it affects $\beta$-D-[methyl- $\left.{ }^{3} \mathrm{H}\right]$ Thiogalactopyranoside $\left(\left[{ }^{3} \mathrm{H}\right] \mathrm{TMG}\right)$ uptake (Opperman et al., 2014).

Despite PA $\beta N$ being defined as an inhibitor, there have been clear indications that AcrB transports PA $\beta N$ out of the cell; studies were done in the wild type strain 3-AG100, using the accumulation properties of PA $\beta \mathrm{N}$ and ethidium to show the transport of these compounds (Figure 33) (Bohnert et al., 2008). However, PA $\beta \mathrm{N}$ is also a well-known inhibitor of AcrB and its homologues, despite it being pumped efficiently out of the cell by AcrB, with a nonlinear, non-hyperbolic uptake curve in dependence of time, as shown by Bohnert et al in 2008 (Bohnert et al., 2008). It was also suggested that PA $\beta \mathrm{N}$ inhibited the efflux of other drugs by binding to the bottom of the distal binding pocket, hydrophobic trap, and also by interfering with the binding of other compounds to the upper part of the binding pocket (Kinana et al., 2016). In this study it has been shown that PA $\beta N$ in the AcrB proteoliposome assay shows the same properties by increasing the basal activity of AcrB in comparison to the substrate taurocholate. All this indicates that $\mathrm{PA} \beta \mathrm{N}$ is a competitive inhibitor of AcrB with substrate behaviour. Since the assay in this study observes this indirectly, by monitoring proton translocation, another investigation should be made, where PA $\beta N$, as well as MBX3132 transport (and substrate transport in general) is monitored in proteoliposomes.

It has to be taken into consideration that substrates of AcrB are quite hydrophobic and this can cause that substrates interact with the membrane of the liposomes. This was observed in some measurements like a decrease in fluorescence of the control liposomes, since this interaction can influence that the membrane becomes more permeable for protons, hence the observed quenching over time (Figure 29, Figure 30). This behaviour is not observed in every measurement, since the quality of the proteoliposomes as well as control liposomes varies from preparation to preparation.

Zgurskaya and Nikaido were first to successfully incorporate AcrB in lipid membranes, and show the activity in vitro (H. I. Zgurskaya \& Nikaido, 1999). For the functional studies of AcrB in liposomes, two strategies were used: The first strategy involves diluting vesicles into a buffer at lower $\mathrm{pH}$ while the second strategy involves the usage of valinomycin. It has been shown that much care has to be taken when valinomycin is used in the transport assay and 
that there is a lack of reproducibility (Picard et al., 2012). Taken that into consideration, this study used the first strategy- dilution into acidic buffer. This gave a good reproducibility that can be further improved.

A homologue of AcrB from P. aeruginosa, MexB, was analysed in proteoliposomes with photo-induced proton gradient that was created with Bacteriorhodopsin (Verchère et al., 2012). Bacteriorhodopsin was a constant source of protons for MexB upon illumination, which was monitored through the change of pyranine fluorescence. The liposomes used in that study were prepared in a similar fashion as in the AcrB proteoliposome assay in the work presented here (4.2.3) which in both cases shown to be a good choice for reproducible results and non-leaky liposomes. All studies mentioned are agreeing on the fact that the RND component is active when reconstituted in liposomes. It appears that AcrA promotes activity of AcrB (H. I. Zgurskaya \& Nikaido, 1999) and MexA does the same for MexB (Verchère et al., 2012), however in the work here that conclusion cannot be made regarding AcrA and AcrB.

Even though the initial conditions have been established for the in vitro investigation of AcrB (this study), here it is suggested the usage of different types of AcrB substrates for further studies. Because of the hydrophobicity of the drugs, in order to avoid their interactions with the liposome membrane, and at the same time have less batch-to-batch variations, usage of radioactively labelled $\beta$-lactams is a suggestion for future experiments.

\subsubsection{Usage of streptavidin-biotin bond for monitoring assembly of the efflux pumps}

The important part of the investigation of any multicomponent system is to have it fully assembled, in order to monitor the transport of the substrates through the pump since only the completely assembled pump can expel the substances outside the living cell.

The method used in this study in order to show the proof of assembly of the components of AcrAB-TolC pump relies on the streptavidin-biotin bond as described (4.2.4). The first study where this approach was taken, Enguéné et al 2015, showed that OprM proteoliposomes interact with MexAB proteoliposomes (Ntsogo Enguéné et al., 2015). 
In this study, the formation of the complex AcrAB-TolC, is clearly shown (Figure 41A, Figure 41B). Formation of the tripartite complex was observed when a $\mathrm{pH}$ gradient was established over the membrane of the proteoliposomes, in the presence of taurocholate, a substrate of the pump. Earlier studies done on AcrB showed that bile salts bind to the protein with higher affinity then the other substrate groups (H. I. Zgurskaya \& Nikaido, 1999). In that manner, no surprise that when used in this assay, taurocholate manages to lock the protein in the conformation that allows binding with the outer membrane protein, $\mathrm{TolC}$, in order to be transported through the pump. When no $\mathrm{pH}$ gradient was applied, but in the presence of taurocholate, the complex formation was observed as well, after immediate pull down. The presence of substrate and/or $\Delta \mathrm{pH}$ promotes the tripartite formation. The complex was observed in the pull-down with $\Delta \mathrm{pH}$ applied, at time zero, as well as after five minutes incubation of the pump components with an acidic buffer (alkaline buffer inside the proteoliposomes). In the absence of substrate and $\Delta \mathrm{pH}$, the complex can be detected after incubation time of one hour. If the complex formation can be detected after shorter incubation time has to be further investigated. Even more prominent bands corresponding to the proteins involved in the complex formation were observed when the pump was incubated with the inhibitor MBX3132 (without pH gradient applied) (Figure 41B), which was shown to be a stable condition also for the in situ studies of the pump (Shi et al., 2019). All this leads to the conclusion that the efflux pump assembly does not require transport activity conditions ( $\mathrm{pH}$ gradient applied). These results are in line with work on the AcrAB-TolC pump from the native membrane, where the inactive variant of AcrB D408A is involved in the complex formation (Tikhonova \& Zgurskaya, 2004). AcrB D408A was co-purified with the fusion protein and outer membrane protein. Energy is not required to assemble AcrABTolC and dissipation of the proton-motive force by ionophores or mutation of essential amino acid only increased the stability of the complex (Tikhonova \& Zgurskaya, 2004). In study presented here, it was additionally shown that the formed complex is stable in time (five minutes), which gives possibilities for tuning the in vitro assay conditions (suggestion for the further optimizations), in order to observe kinetics of the substrate transport.

One interesting observation can be made and that is that AcrB can interact directly with TolC in vitro, without the presence of the membrane fusion protein. This fact was discussed in several studies of several laboratories across the world. In one study, it was noticed that AcrB and TolC cannot form a stable complex in vitro, shown by ITC measurements (Touzé et al., 2004). Another study showed, that the AcrB-TolC complex may be formed through a two- 
step mechanism via transient tip-to-tip interaction of AcrB and TolC (Tamura et al., 2005). The periplasmatic (total) length of AcrB and of TolC is $17 \mathrm{~nm}$, which correspond to around the shortest periplasmatic width (Matias et al., 2003). This can explain the hypothesis about tip-to-tip interaction between AcrB and TolC. The TolC docking domain at the top of AcrB fits to how it looks like when the trimer of the AcrB is docked to the TolC trimer. More recent investigation was done by a molecular dynamics simulation approach, where in five out of six stimulations it was observed that direct contact between TolC and docking domain of AcrB can happen. This interaction was stable only when both proteins are docking in tipto-tip manner. TolC was closed at the inner periplasmatic bottleneck region, which suggest that either longer time or some other additional components are needed in order for TolC and AcrB to interact (Schmidt et al., 2016). The work presented here shows that interaction between proteoliposomes containing either AcrB or TolC occurred when a substrate of the pump was present, without the necessity for a proton motive force, and in both situations when AcrB or TolC proteoliposomes were labelled with biotin.

In the light of the recent data obtained by Cryo-EM (Shi et al., 2019), where it was observed that AcrB and TolC do not interact in situ, the observations from the study presented here have to be further investigated. One major difference between the two studies ((Shi et al., 2019); this work) is that one uses cells and the other proteoliposomes. The usage of proteoliposomes and purified protein results in a much higher protein concentration thus increasing the chance for protein-protein interactions. Those interactions can be unspecific thus additional controls are necessary (e.g., proteoliposomes containing a protein that is not an interaction partner of AcrB).

The obtained data indicate that in vitro interaction between the components of the pump $\mathrm{Acr} A \mathrm{~B}$ and TolC is present in a number of conditions, making this approach of assembly through incubation of proteoliposomes, highly recommended for further transport studies.

\subsubsection{TolC wild type in a closed state is an essential part for studying the substrate transport}

The apo-form of the pump AcrAB-TolC with a closed TolC represents a resting state (Wang et al., 2017). At the same time, the pump assemblies with opened TolC in the presence of 
transportable ligands represents transport states with the AcrB trimer in different conformations (LLT, LTT or LTO) (Wang et al., 2017). It was proposed that substrate binding in the apo state initiate quaternary structural changes in AcrB that are communicated to AcrA which then triggers tertiary structural changes in TolC in order to open the channel from a sealed resting state (Wang et al., 2017). The same conclusion was made when this pump was observed in situ (Shi et al., 2019). In the presence of antibiotics, the AcrAB subcomplex changes conformation to recruit TolC, which stays closed in the outer membrane to keep the periplasm isolated from the extracellular environment.

TolC variants used in this study were: the wild type and YFRS "open" variant. Indeed, previously described studies done on YFRS TolC indicated that there is significant destabilization of the tunnel's closed state; at the positive potentials the TolC YFRS high conductance state was stable, above $-60 \mathrm{mV}$ it became highly unstable, the lifetime of the open state was short and there were frequent switches into several conductance states including complete closure (Andersen et al., 2002). The strong amplification at positive potentials could reflect the strong net negative charge at the entrance enhancing the opening at high positive potentials. This effect would be expected to hold the entrance in the stable open conformation (Andersen et al., 2002).

In this study, the difference in the states between TolC wild type and the open mutant TolC, are shown. TolC wild type does not show a decrease in the fluorescent signal of pyranine, which does not exit the lumen of the liposomes through TolC, while the same cannot be said for TolC YFRS liposomes. These liposomes show significant decrease in the fluorescence (Figure 45), indicating that pyranine can exit through the variant. Another point to address here: Why the signal of the mutant is not closer to zero, since TolC is in open state and the dye can freely diffuse out of the liposome? This pyranine assay can be used for showing the reconstitution efficiency of TolC in liposomes and if this is taken into consideration it can be said that the signal for the TolC YFRS liposomes cannot reach the zero since not every liposome contains the protein. This knowledge, either about the state or about the reconstitution efficiency, is important as a control of the qualitative characteristics of produced protein and produced proteoliposome samples at the same time.

TolC in the apo-form of the pump is in the closed state, and it needs the other components of the pump, the inner membrane protein and the membrane fusion protein, in order to transition into the open state. That is why for the studies of the substrate transport, it would be 
important to use TolC wild type, for the representation of the native state of the protein situated in the outer membrane.

\subsubsection{Efficiency of the protein incorporation into the membrane}

To know the right amount of the detergent to be used for the solubilization of the liposomes is the key for the successful incorporation of membrane protein inside the vesicle, when detergent-mediated method for reconstitution is used. Three stages of solubilization are in detail described in 1.7.4. In short, three different phases are present during solubilization of the liposomes: start of the solubilization, mixture of the solubilized liposomes and detergent micelles and total solubilization of liposomes. The phase that should be used in most cases is the mixture between solubilized liposomes and detergent micelles. On Figure 43, it is shown how the solubilization process looks like for the liposomes prepared for the usage in AcrB proteoliposomes assay, and for the in vitro assembly assay. Both liposome preparations were treated with $\beta-\mathrm{OG}$ and the absorbance signal of the lipid mixture was monitored. For both preparations, one with DOPC/Cholesterol lipids, and the other with DOPC/Cholesterol/DOPE-biotin cap, showed similar behaviour when solubilization took place. It can be concluded that DOPE-biotin cap lipid addition did not make influence on the start of the onset solubilization and at the same time the concentration of the detergent to be used for protein incorporation did not vary between the assays. Adding cholesterol to DOPC in the preparation of the liposomes is said to increase the transition temperature of the resulting mixture. At the same time, even though not present in bacteria, cholesterol is used for liposome preparation since it makes liposomes less leaky, which is an enormously important when designing a transport assay. Additionally, when cholesterol is used in the adequate ratio, liposomes could be exposed to the temperature of $4^{\circ} \mathrm{C}$ without phase transition, which is important since proteins are being manipulated at the same temperature (Verchère, 2014).

The prepared proteoliposomes were subjected to detergent solubilization followed by a centrifugation step. The purpose of this assay is to provide information about how much protein is correctly incorporated in the liposomes (sample after centrifugation), in compression to the amount that are both correctly and incorrectly incorporated (aggregates) 
in the liposomes (sample before centrifugation) (Figure 44). The SDS-PAA gel stained with Coomassie solution was analysed with Image J (Schneider et al., 2012). The bands were compared with one another for the purpose of calculating the incorporation efficiencies (Table 15). When the bands presenting the proteoliposome samples were compared with purified protein samples, the estimation of the protein amounts in liposomes can be made (Table 16) (Figure 44). According to the data obtained, reconstitution efficiencies vary from protein to protein, between $54 \%$ and $98 \%$ (except for AcrA proteoliposomes, around $4 \%$ ). This leads to the conclusion that the detergent-mediated reconstitution can be used for most proteins used in the in vitro AcrB proton-drug transport assay. It is noteworthy, that the reconstitution efficiency seems to increase for proteins with larger hydrophobic domains compared to the soluble domains (Figure 7). AcrA has the lowest reconstitution efficiency ( $4 \%$ ) and is only attached to the membrane with a lipid anchor, meaning that the most of the protein is soluble (1.6.1). AcrB on the other hand is embedded in the membrane for a large part (about $31 \%$ of membrane embedded residues) and shows the highest reconstitution efficiency (98\%). For TolC, the reconstitution efficiency was between efficiencies of AcrA and AcrB (54\%) while at the same time it is embedded in the membrane (about $9 \%$ of membrane embedded residues) but also has a large soluble domain.

Even though the efficiency of the protein incorporation is the immediate sign of the condition of the sample, it does not necessarily tell the quality level. Especially when the orientation of the proteins in the liposomes is not known. It is important to maximize the extent of the incorporation of the protein in the liposomes. A higher rate of protein incorporation results in a better proteoliposome sample that can be used in further experiments. 


\section{Chapter V: Silver-ion resistance in Escherichia coli is mediated by the efflux pumps CopA and CusCBA}

\subsection{The use of P-type ATPase CopA as proof-of-principle for silver-ion transport observations}

\subsubsection{Introduction}

The P-type ATPase CopA was used for establishing an assay in which the transport of metal ions can be observed. CopA is a member of a subfamily that transports the cations of soft Lewis acids (soft metal cations) such as $\mathrm{Cu}(\mathrm{I})$ and $\mathrm{Ag}(\mathrm{I})$ (Stoyanov et al., 2003)(Odermatt et $a l .$, 1993). The ions are being transported across cell membranes with energy derived from ATP hydrolysis (Wijekoon et al., 2017). This scenario is depicted in Figure 47.

When reconstituted into the liposomes, inside-out oriented CopA can be triggered for the transport of metal ions into the proteoliposomes. This is achieved by addition of the substrate (metal ions) as well as ATP on the outside of the proteoliposome. Addition of ATP catalyses the beginning of the reaction and silver ions are pumped into the lumen of the proteoliposome. If the fluorescent dye Phen Green SK (PGSK) is present on the inside of the proteoliposomes, the accumulation of the metal ions can be detected by the quenching of the fluorescent signal upon binding of the metal ions to the dye (Figure 47).

This setup can also be used for monitoring more complex transporters, like the tripartite $\mathrm{Ag}^{+} / \mathrm{Cu}^{+}$-exporting CusCBA system (5.2). 


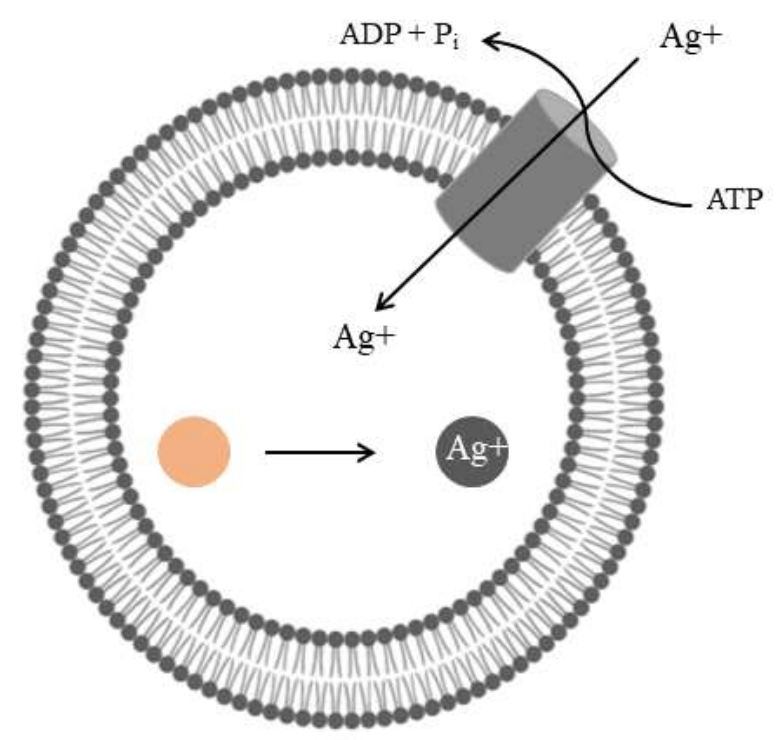

Figure 47. CopA, a P-type ATPase, expels silver and copper ions from the cytoplasm of Gram-negative bacteria. The bilayer of the liposomes represents the inner membrane. CopA that is in the orientation inside-out, will be triggered by the addition of ATP to transport silver ions. Silver ions present in the buffer outside the proteoliposomes, will be transported to the inside; the dye PGSK (orange circle) $(\lambda \mathrm{ex}=480 \mathrm{~nm} ; \lambda \mathrm{em}=530 \mathrm{~nm}$ ) binds metal ions and its fluorescence will be quenched (grey circle).

\subsubsection{Results}

\subsubsection{Production of $\operatorname{CopA}$ wild type for the functional analysis}

CopA wild type was used in this study, and for the purpose of production, the gene encoding wild type E. coli CopA was amplified from the E. coli genome and cloned into the p7XC3H vector and expressed as a C-terminal His-tag variant using IPTG as an inducer in the T7based expression host E. coli C43(DE3) $\triangle a c r A B D$ (3.2.2.3). As shown on Figure 48, the protein was purified with high purity, shown on Coomassie-stained SDS-PAA gel, resulting in a monodisperse peak at $15.3 \mathrm{ml}$ on the SEC profile.

A few additional lower molecular weight bands are visible in the fractions after $\mathrm{Ni}^{2+}$-NTA affinity purification as well as after SEC, even in the constant presence of the reducing agent, $\beta$-mercaptoethanol. These additional bands were also observed in experiments with CopA having C-terminal histidine tag by Fan and Rosen in 2002 (Fan \& Rosen, 2002). They 
proposed that the proteins with the higher electrophoretic mobility interacted with the antiHis antibody are degradations at the $\mathrm{N}$-terminus of the protein.

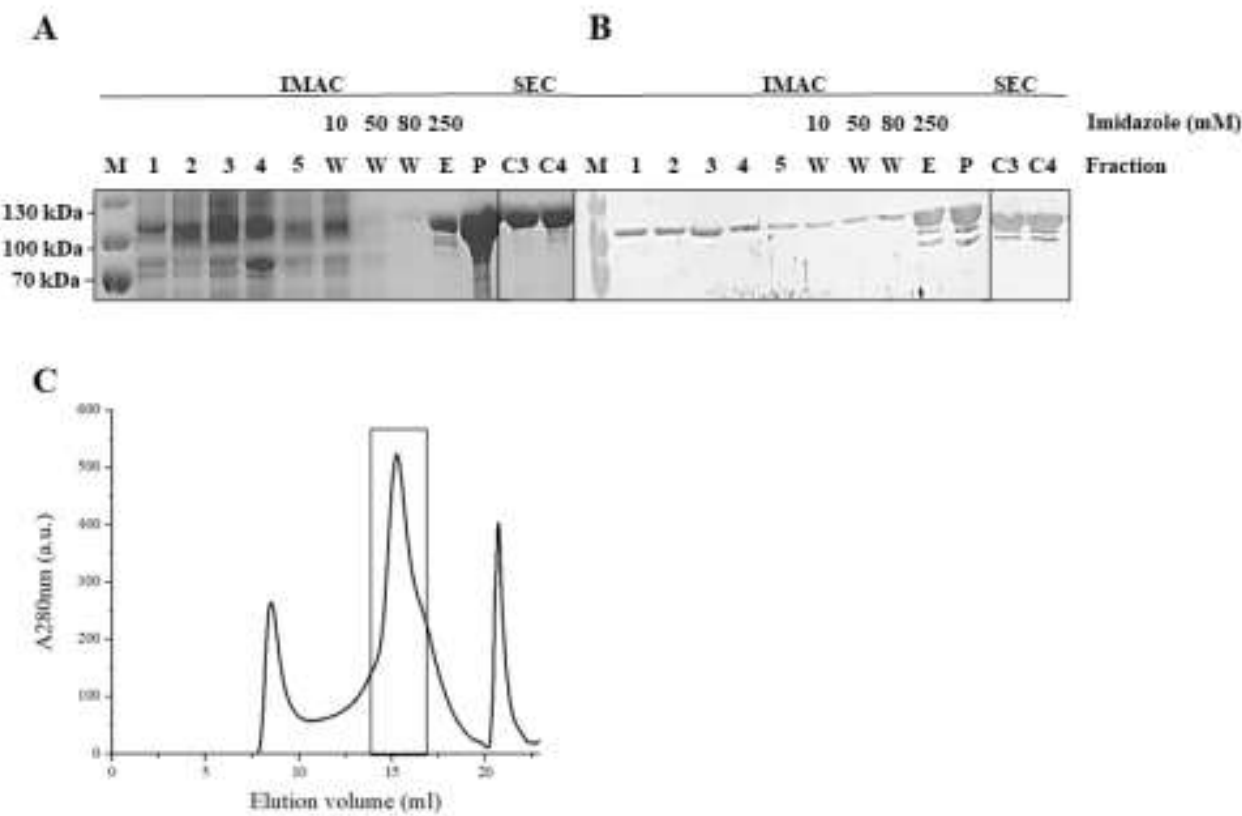

Figure 48. Optimized purification of CopA wild type. A. Coomassie-stained 10\% SDS-PAA gel of purified CopA wild type. B. Western Blot analysis with identical samples from A. The Western Blot was probed with monoclonal anti-polyHistidinealkaline phosphatase produced in mouse (Sigma A5588). M-Marker (PageRuler Prestained Protein Ladder (ThermoScientific)), 1-Membrane, 2-Solubilized membrane, 3-Supernatant, 4-Pellet, 5-Flow through, W-samples from indicated wash steps from $\mathrm{Ni}^{2+}$-NTA purification, E-Eluate, P-Concentrated protein sample after $\mathrm{Ni}^{2+}$-NTA purification, C4/C5-Samples from the fractions collected after SEC. Applied: 1-P (samples were diluted 12.5x in SDS-sample buffer) $10 \mu \mathrm{l}$ for Coomassie, $5 \mu \mathrm{l}$ for Western blot; P- $10 \mu \mathrm{g}$ for Coomassie, $2 \mu \mathrm{g}$ for Western blot; C3/C4- $5 \mu \mathrm{g}$ for Coomassie, $2 \mu \mathrm{g}$ for Western blot. C. Size exclusion chromatography profile of a $\mathrm{Ni}^{2+}$-NTA purified sample of CopA wild type. The protein was purified on a Superose 6 Increase 30/100 column equilibrated with $20 \mathrm{mM} \mathrm{HEPES} \mathrm{pH} \mathrm{7.5,} 5 \mathrm{mM} \mathrm{MgSO} 4,1 \mathrm{mM} \beta$ mercaptoethanol, $10 \%$ glycerol, $0.2 \%$ PMSF, $0.05 \%$ DDM, with flow rate $0.4 \mathrm{ml} / \mathrm{min}$ and peak was detected at $15.3 \mathrm{ml}$.

\subsubsection{CopA ATPase activity}

After purification, it is important to test the activity of the enzyme in the solubilized state before it is used for reconstitution and activity studies in liposomes. The NADH-coupled ATPase assay has long been used to measure the ATPase activity of various enzymes, such as the sarcoplasmic reticulum $\mathrm{Ca}^{2+}$ pump (Warren et al., 1974). The NADH-coupled ATPase assay is based on a reaction in which the regeneration of hydrolyzed ATP is coupled to the oxidation of NADH (Figure 49). Upon ATP hydrolysis, ATP is regenerated via phosphoenolpyruvate in the presence of pyruvate kinase, yielding equimolar amount of 
pyruvate. Lactate dehydrogenase, also present in the assay, reduces pyruvate to lactate retrieving the electrons from $\mathrm{NADH}$ which is oxidized to $\mathrm{NAD}^{+}$. The oxidation of NADH in the solution is continuously measured at a wavelength of $340 \mathrm{~nm}$. Under the assumption that the ATP hydrolysis is the rate limiting step in this coupled assay, the NADH oxidation rate reflects the ATP hydrolysis rate (Figure 50).

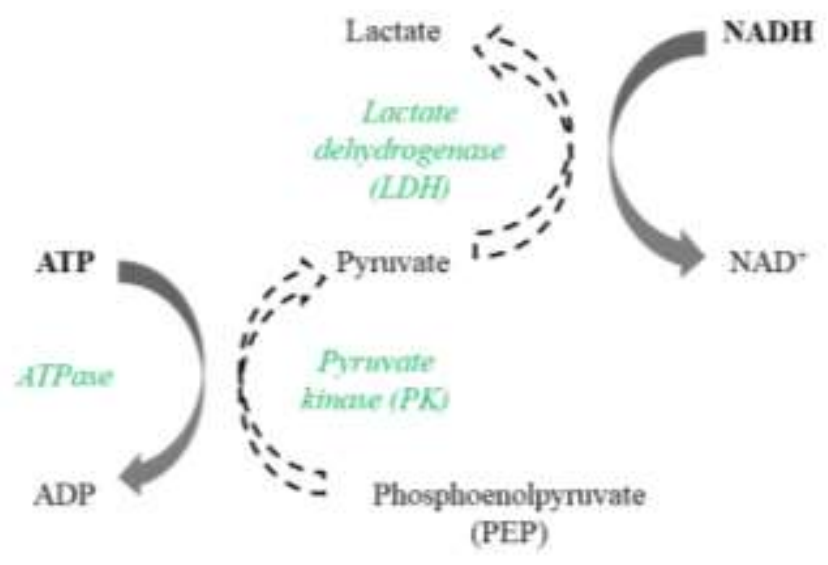

Figure 49. The ATP-NADH assay is based on a reaction in which ATP hydrolysis is coupled with the oxidation of NADH. The decrease in absorbance of NADH, observed at $340 \mathrm{~nm}$, is proportional to the ATP hydrolysis rate. The reaction mixture contains, besides the ATPase, phosphoenolpyruvate and pyruvate kinase, as well as lactate dehydrogenase.

The assay is performed at different concentrations of ATP and in the presence of $20 \mu \mathrm{M}$ silver nitrate. This resulted in a $\mathrm{K}_{\mathrm{m}}$ of $3.94 \pm 2.5 \mathrm{mM}$ and $\mathrm{a} \mathrm{V}_{\max }$ of $256.51 \pm 91.47$ $\mathrm{nmol} / \mathrm{min} / \mathrm{mg}$ protein (Figure 50Figure 49) in comparison to the values obtained by Fan et al, in the presence of copper ions where $K_{m}$ was $0.52 \pm 0.05 \mathrm{mM}$ and $V_{\max } 190 \pm 6 \mathrm{nmol} / \mathrm{min} / \mathrm{mg}$ protein (Fan \& Rosen, 2002) (Table 18). 


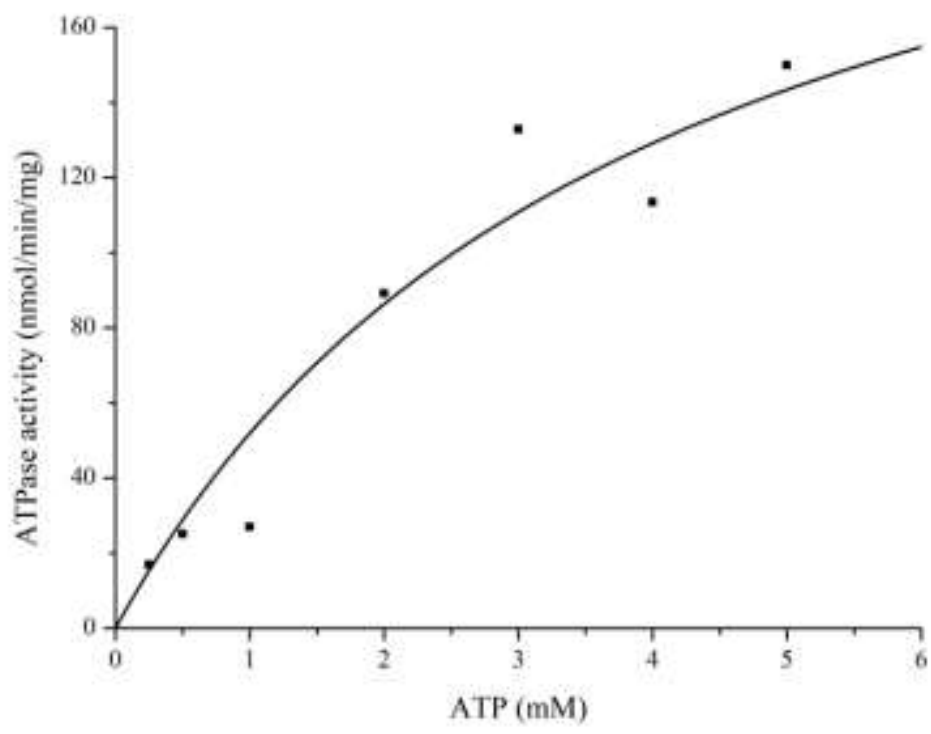

Figure 50. The kinetics of the ATPase activity of purified CopA in detergent micelles. The ATPase activity of purified ATPase was determined in the presence of the indicated concentrations of ATP. The assay was performed in the presence of $20 \mu \mathrm{M}$ silver nitrate. The data was fitted to the Michaelis-Menten equation using Origin (Origin 8.6. OriginLab Corporation, Northampton, MA, USA.) yielding a $\mathrm{K}_{\mathrm{m}}$ of $3.94 \pm 2.5 \mathrm{mM}$ and a $\mathrm{V}_{\max }$ of $256.51 \pm 91.47 \mathrm{nmol} / \mathrm{min} / \mathrm{mg}$ protein.

Table 18. The kinetic parameters of the ATPase activity of CopA, gathered during ATP-NADH assay.

\begin{tabular}{|l|l|}
\hline Parameters & Value \\
\hline $\mathrm{V}_{\max }(\mathrm{nmol} / \mathrm{min} / \mathrm{mg})$ & $256.51 \pm 91.47$ \\
$\mathrm{~K}_{\mathrm{m}}(\mathrm{mM})$ & $3.94 \pm 2.5$
\end{tabular}

\subsubsection{Phen Green $\mathrm{SK}^{\mathrm{TM}}$ as a reporter system}

The fluorescent probe Phen Green SK ${ }^{\mathrm{TM}}$ (PGSK) is a member of the photoinduced electron transfer (PET) sensors (Figure 51). Metal ions bind to it by a specific ligand which is covalently attached to the fluorophore, which comprises a phenanthroline and a fluorescein derivate moiety (Kim \& Ahner, 2006). It exhibits its maximum of fluorescence when it is in the free form. The fluorescence decreases following complexation with a metal ion. 


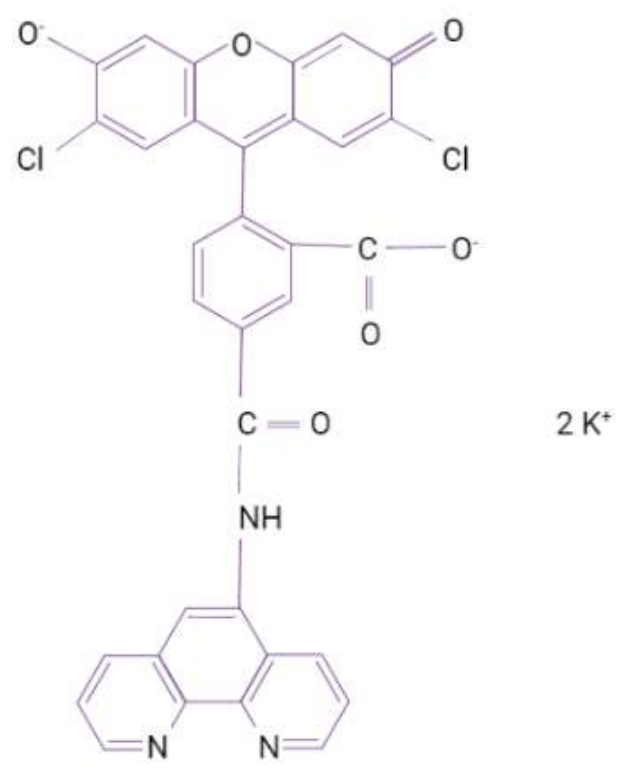

Figure 51. Structure of Phen Green SK ${ }^{\mathrm{TM}}$, PGSK. Figure was made with help of BioRender (https://app.biorender.com/).

A study done with $\mathrm{Cu}(\mathrm{I})$ and PGSK shows stoichiometry of 1:1 for the binding of copper ions. The same study shows that the exchange of $\mathrm{Cu}(\mathrm{I})$ with PGSK is slow since it takes almost 2 hours for the PGSK to stabilize (Kim \& Ahner, 2006).

Not so many data are available about the behaviour of PGSK with silver ions. But some data are available about $\mathrm{Cu}(\mathrm{I})$ and PGSK, and since $\mathrm{Cu}(\mathrm{I})$ and $\mathrm{Ag}(\mathrm{I})$ belong to the same group (I B) of elements, it was assumed that the influence of $\mathrm{Ag}(\mathrm{I})$ is similar to the one of $\mathrm{Cu}(\mathrm{I})$. Additionally, the dye was used in a study of CusA activity in vitro (Long et al., 2010). Taking everything into consideration (previous study, as well as similarity with copper), it was chosen for this study as well, for both CopA and CusA functional assays.

When investigating the activity of a protein in vitro, it is important to choose the right reporter system. For the observation of the activity of CopA as well as CusA, two different reporter systems were used - ACMA and PGSK. Usage of different dyes goes with the fact that different characteristics of the transport properties of the protein should be investigated.

Three concentrations of PGSK, $200 \mu \mathrm{M}, 1 \mu \mathrm{M}$ and $200 \mathrm{nM}$, were tested before usage in the proteoliposomes assay, as shown in Figure 52A. For each aforementioned concentration, the dye was titrated with silver nitrate, in order to test the behaviour in the presence of this compound, since the same was used in the proteoliposome assay as the source of the silver ions. The concentration of silver nitrate was tested in the range between $50 \mu \mathrm{M}$ and $1 \mathrm{mM}$. Quenching maximum was reached at $200 \mu \mathrm{M}$ silver nitrate. A closer look was taken in the 
concentration range between $50 \mu \mathrm{M}$ till $200 \mu \mathrm{M}$ silver nitrate at a PGSK concentration of $200 \mathrm{nM}$. In that case, the maximum quenching was reached at $100 \mu \mathrm{M}$ (Figure 52B). Further addition of silver nitrate did not show any notable change in quenching.

A)

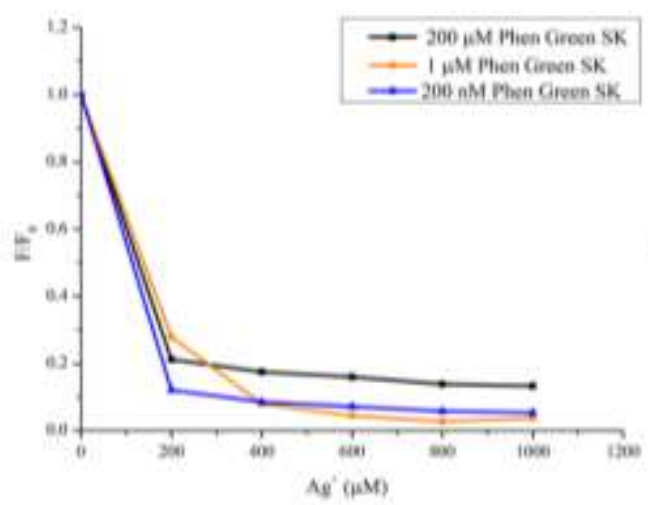

B)

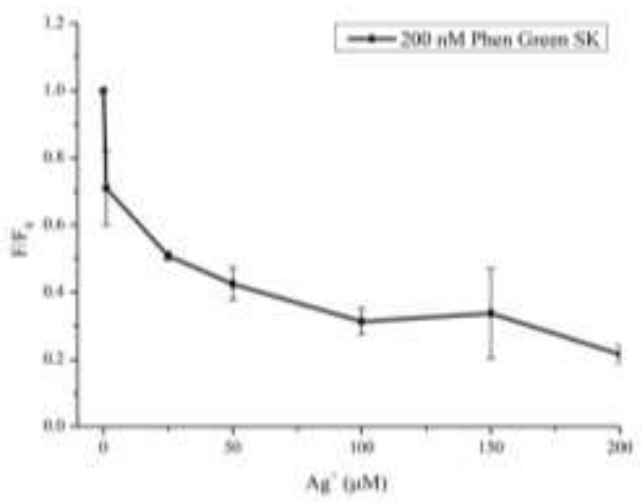

Figure 52. Phen Green SK dye was titrated with different concentrations of silver nitrate. PGSK was tested in a buffer $2 \mathrm{mM}$ HEPES pH 7.5, $100 \mathrm{mM} \mathrm{K}_{2} \mathrm{SO}_{4}$. A) Different concentrations of PGSK were titrated with different concentrations of silver nitrate. B) PGSK at $200 \mathrm{nM}$ titrated with different concentrations of silver nitrate. Data points are average of three consecutive measurements $(n=3)$. Fluorescence was measured at $\lambda_{\mathrm{ex}}=480 \mathrm{~nm} ; \lambda_{\mathrm{em}}=530 \mathrm{~nm}$. Fluorescence was normalized to fold change, $\mathrm{F} / \mathrm{F}_{0}$ (division by the initial value).

As shown on Figure 52B, the fluorescent signal of 200 nM Phen Green SK gets almost $60 \%$ quenched by addition of $50 \mu \mathrm{M}$ of silver nitrate. Since for the assay $1 \mathrm{mM}$ of silver nitrate with $200 \mu \mathrm{M}$ dye was used (3.2.2.20), in the case when $100 \%$ of PGSK is incorporated inside the proteoliposomes, it will be quenched almost $95 \%$ (Figure 52A). 


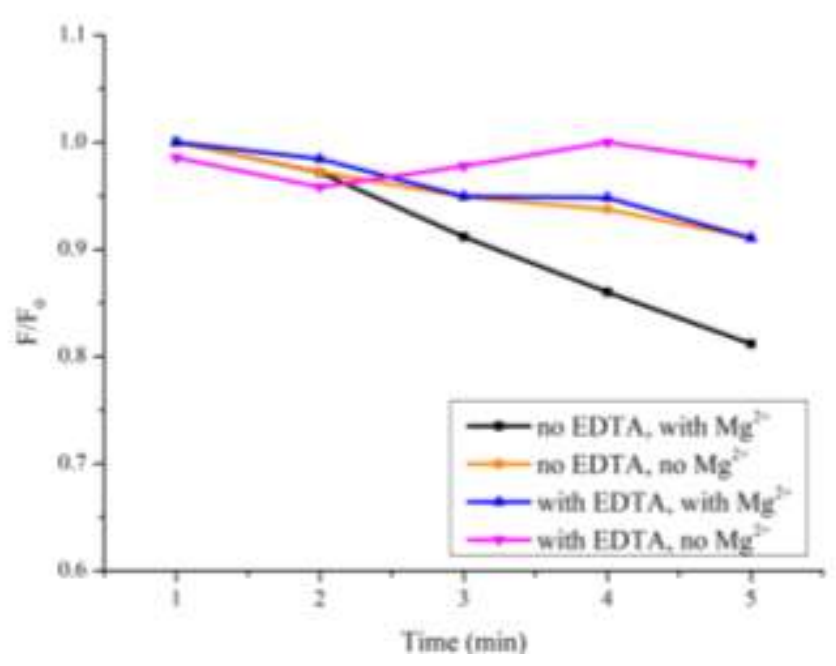

Figure 53. Fluorescence of PGSK is quenched by magnesium ions. PGSK was suspended in buffer 2 mM HEPES pH 7.5, $100 \mathrm{mM} \mathrm{K}_{2} \mathrm{SO}_{4}$. When magnesium ions are chelated by EDTA, fluorescent signal is stable for at least five minutes. Fluorescence was measured at $\lambda_{\mathrm{ex}}=480 \mathrm{~nm} ; \lambda_{\mathrm{em}}=530 \mathrm{~nm}$. Fluorescence was normalized to fold change, $\mathrm{F} / \mathrm{F}_{0}$ (division by the initial value).

It was observed that PGSK is quenched in the presence of magnesium ions (Figure 53). When the buffer ( $2 \mathrm{mM}$ HEPES $\mathrm{pH} 7.5,100 \mathrm{mM} \mathrm{K}_{2} \mathrm{SO}_{4}$ ) did not contain magnesium ions (in a form of $\left.\mathrm{MgCl}_{2}, 5 \mathrm{mM}\right)$ or in the presence of the chelator, EDTA $(0.1 \mathrm{mM})$, the fluorescent signal was stable for at least five minutes.

\subsubsection{Solubilization assay to validate the reconstitution of CopA wild type into artificial membranes}

The CopA wild type proteoliposomes were at first prepared via detergent solubilization for the protein incorporation and subsequent Bio-Beads-mediated removal of the used detergent. Different protein to lipid ratios were tested for the efficiency of the protein incorporation into the liposomes. The performed assays with CopA reconstituted in the liposomes via detergentmediated reconstitution showed not to be the best method, which was concluded when the sample quality was checked and the performed proteoliposome assay was not reproducible (data not shown). Taken that into the consideration, a different reconstitution method was taken. CopA was incorporated in liposomes via mechanical reconstitution, by performing several rounds of freeze and thaw cycles. PGSK was incorporated in the vesicles during the reconstitution. Samples before and after ultracentrifugation upon detergent solubilization 
were compared (Figure 54) using ImageJ (Schneider et al., 2012) and according to the calculated band intensities, the efficiency of the correctly incorporated protein was estimated. The band's intensities before and after ultracentrifugation are compared with a sample of purified protein of known concentration.

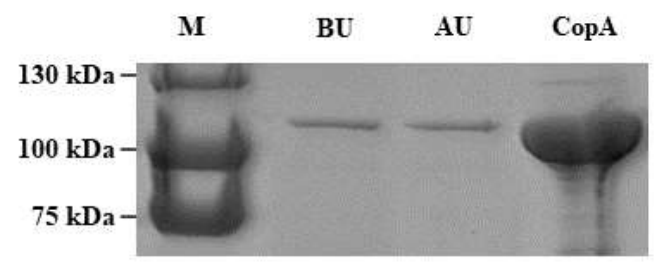

Figure 54. Solubilization assay of CopA wild type proteoliposomes, prepared for usage with ACMA. Coomassie-stained $10 \%$ SDS-PAA gel of samples obtained during solubilization assay. The protein was reconstituted for two different assays, where two different dyes were used. When 9-amino-6-chloro-2-methoxyacridine (ACMA) dye was used as a reporter system, the dye was added directly before the measurements start. M-Marker (PageRuler Prestained Protein Ladder (ThermoScientific)). BU and AU- Samples before and after ultracentrifugation, respectively. For the calculation of the protein amount in liposomes, the sample of the purified protein was loaded on the SDS-PAA gel, CopA- $6.4 \mu \mathrm{g}$.

The efficiency of the correct incorporation of the protein in liposome was examined for CopA proteoliposome preparation (Figure 54). CopA proteoliposomes prepared with PGSK showed $98 \%$ of correctly incorporated protein. The reconstitution efficiency was calculated as well, as a percentage of protein incorporated in liposomes compared to the amount of protein added in the beginning of reconstitution (Table 19). Image $J$ is used for comparison of the band intensities (Schneider et al., 2012) and estimation of protein amounts in proteoliposomes (Table 19).

Table 19. Protein amount in the CopA wild type proteoliposomes. Protein amount used in the activity assay (100 $\mu$ of the sample used in each activity assay) is shown. This amount was evaluated according to the Image J (Schneider et al., 2012) estimations of the band intensities before and after ultracentrifugation as shown in the Figure 54 and subsequent comparison with the band of the sample of purified protein of known concentration.

\begin{tabular}{|l|l|l|}
\hline Assay type & $\begin{array}{l}\text { Reconstitution efficiency } \\
(\boldsymbol{\%})\end{array}$ & $\begin{array}{l}\text { Protein amount used in the activity } \\
\text { assay }(\boldsymbol{\mu g})\end{array}$ \\
\hline PGSK & $32 \%$ & 3.6 \\
\hline & & \\
\hline
\end{tabular}




\subsubsection{Phen Green SK as a reporter system for the transport of silver ions by the P-type ATPase CopA}

As shown in Figure 47, the assay for observation of silver ion transport through CopA requires that both silver ions and ATP are added in the buffer outside the proteoliposome. With that approach, the ions transported inside the lumen would be binding to the metal-ion sensitive dye, PGSK, and the fluorescence of the dye would be quenched.

Figure 55 shows the activity of CopA reconstituted in the liposomes. $5 \mathrm{mM}$ ATP was added to the proteoliposomes to trigger the transport of silver ions, which were present in the buffer outside the vesicle (20 mM HEPES/NaOH pH 7.6, $50 \mathrm{mM} \mathrm{K}_{2} \mathrm{SO}_{4}, 10 \mathrm{mM} \mathrm{MgSO}$ ) as $0.5 \mathrm{mM} \mathrm{AgNO} 3$.

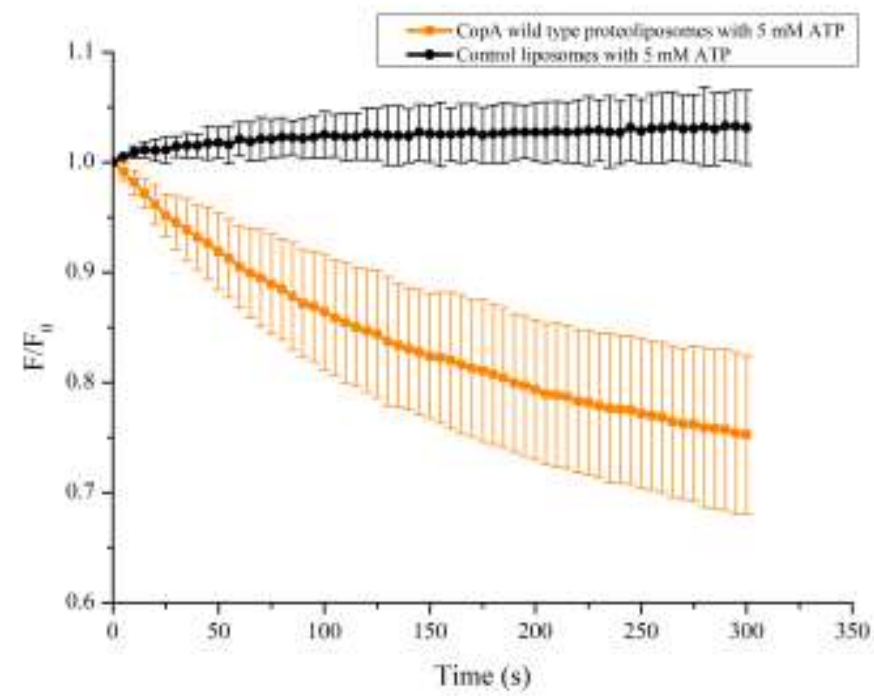

Figure 55. CopA reconstituted in liposomes shows activity. Fluorescence of PGSK dye was monitored as a function of time. Fluorescence was measured at $\lambda_{\mathrm{ex}}=410 \mathrm{~nm} ; \lambda_{\mathrm{em}}=500 \mathrm{~nm}$. Fluorescence was normalized to fold change, $\mathrm{F} / \mathrm{F}_{0}$ (division by the initial value). Data points are the average of three consecutive measurements $(n=3)$.

Quenching of PGSK (Figure 55, orange line) represents the binding of silver ions entering the proteoliposomes via CopA, which represents the activity of this enzyme. For the control, empty liposomes were used (Figure 55, black line). This control liposomes were subjected to the same conditions as CopA proteoliposomes; $5 \mathrm{mM}$ ATP was added to the solution. 
Opposite to CopA proteoliposomes, control liposomes that do not contain the enzyme, show no quenching of the dye, meaning that silver ions do not cross the membrane of the liposomes.

Sodium azide was used as an additional control, to show the behaviour of the active enzyme in comparison to the one treated with this compound. When sodium azide was used in order to inhibit the activity of CopA in detergent micelle, it was noticed that ATP hydrolysis mediated by CopA was slower than the when sodium azide was not used (data not shown). Fan and Rosen notice in their experiments that CopA-mediated hydrolysis was inhibited 8090\% by sodium azide (Fan \& Rosen, 2002). CopA proteoliposomes, as well as control liposomes, treated with $1 \mathrm{mM}$ sodium azide are shown in Figure 56 (magenta and olive line, respectively). In the presence of the sodium azide, proteo(liposomes) are not showing quenching as the one observed in the presence of ATP; however, the quenching present in this case is the same within proteoliposomes and empty liposomes, indicating that sodium azide interacts with liposome's membrane and makes PGSK accessible to silver ions. Orange and black curves are the identical data as the one from Figure 55.

When CopA is reconstituted inside the bilayer of the liposomes, but silver ions are not present in the buffer outside the liposomes, CopA does not show activity upon addition of ATP, shown as a minor quenching reaction compared to the one in the presence of silver ions (Figure 57, blue and orange line, respectively). For comparison, curve corresponding to CopA proteoliposomes in the presence of sodium azide is shown (Figure 57, pink line). Orange and pink curves are identical data from the Figure 55 and Figure 56, respectively. 


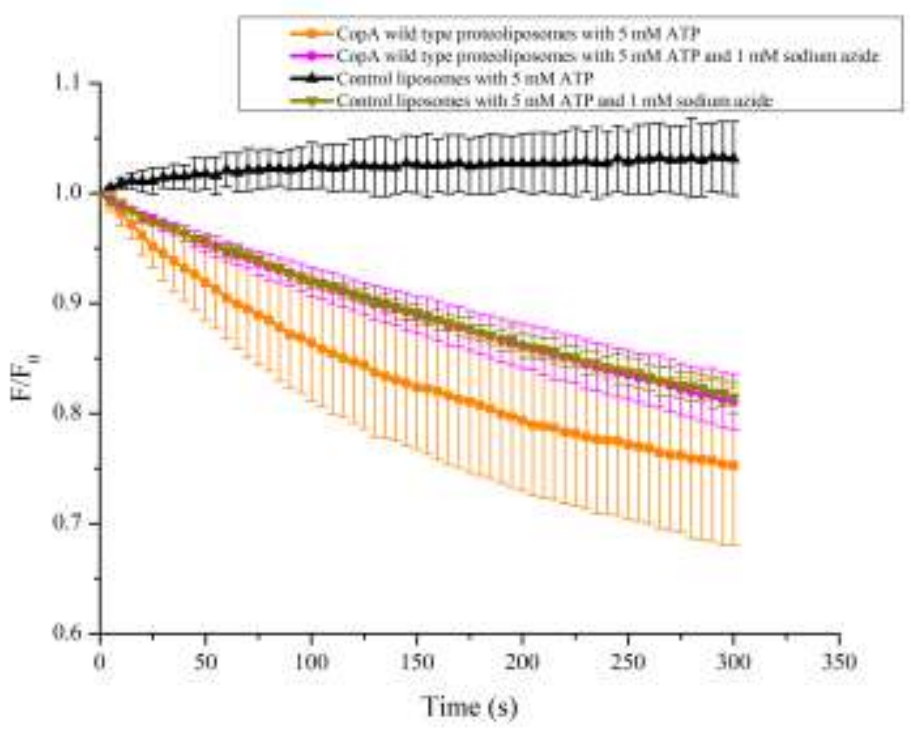

Figure 56. CopA reconstituted in liposomes in the presence of sodium azide. Fluorescence of PGSK dye was monitored as a function of time. Fluorescence was measured at $\lambda_{\mathrm{ex}}=410 \mathrm{~nm} ; \lambda_{\mathrm{em}}=500 \mathrm{~nm}$. Fluorescence was normalized to fold change, $\mathrm{F} / \mathrm{F}_{0}$ (division by the initial value). Data points are the average of three consecutive measurements $(\mathrm{n}=3)$.

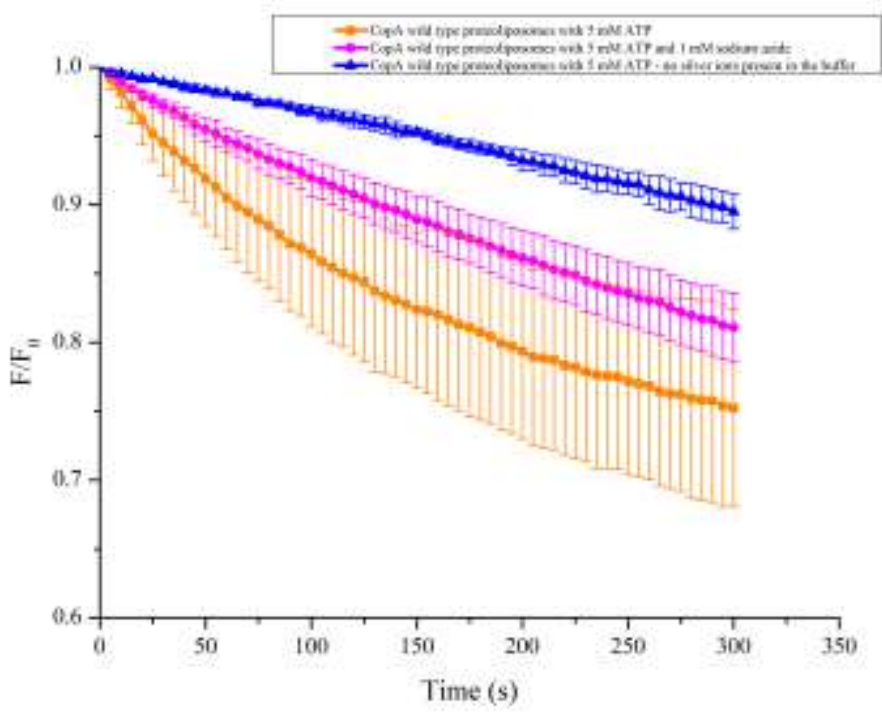

Figure 57. CopA reconstituted in liposomes does not show substantial quenching of PGSK in absence of silver ions. Fluorescence of PGSK was monitored as a function of time. Fluorescence was measured at $\lambda_{\mathrm{ex}}=410 \mathrm{~nm} ; \lambda_{\mathrm{em}}=500 \mathrm{~nm}$. Fluorescence was normalized to fold change, $\mathrm{F} / \mathrm{F}_{0}$ (division by the initial value). Data points are the average of three consecutive measurements $(n=3)$. 


\subsubsection{Discussion}

\subsubsection{Produced CopA is active in detergent micelles}

In order to prove that CopA, produced in this study, behaves as other known members of the P-type ATPase superfamily, the activity of the enzyme was investigated in the presence of substrate $(20 \mu \mathrm{M}$ silver nitrate).

The data in this study demonstrates that CopA from E. coli in the presence of $20 \mu \mathrm{M} \mathrm{Ag}$ (I) yielded an ATP $\mathrm{K}_{\mathrm{m}}$ of $3.94 \pm 2.5 \mathrm{mM}$ and $\mathrm{V}_{\max }$ of $256.51 \pm 91.47 \mathrm{nmol} / \mathrm{min} / \mathrm{mg}$ protein.

Previous studies done with CopA from E. coli were analysing the behaviour of the enzyme in the presence of copper. The data resulted in values of $\mathrm{K}_{\mathrm{m}}$ of $0.52 \pm 0.05 \mathrm{mM}$ and $\mathrm{V}_{\max }$ of $0.19 \pm 0.06 \mu \mathrm{mol} / \mathrm{min} / \mathrm{mg}$ protein (Fan \& Rosen, 2002). A recent study exhibited a $\mathrm{V}_{\max }$ of $7.73 \pm 0.19 \mathrm{nmol} / \mathrm{mg} / \mathrm{min}$ and $\mathrm{K}_{\mathrm{m}}$ of $0.11 \pm 0.02 \mu \mathrm{M}$ for E. coli CopA in the presence of $\mathrm{Cu}(\mathrm{I})$ (Abeyrathna et al., 2020). When the homologue of E. coli CopA from the thermophilic, sulphur metabolizing Archaeoglobus fulgidus was studied, the data about the behaviour of the enzyme obtained in the presence of silver, yielded in an ATP $\mathrm{K}_{\mathrm{m}}$ of $0.25 \pm 0.04 \mathrm{mM}$ and $\mathrm{V}_{\max }$ of 12 to $14.5 \mu \mathrm{mol} / \mathrm{h} / \mathrm{mg}$ (200 - $241.67 \mathrm{nmol} / \mathrm{min} / \mathrm{mg}$ ) (Mandal \& Argüello, 2003). The same study also discovered that the enzyme displays a smaller $\mathrm{V}_{\max }$ in the presence of $\mathrm{Cu}(\mathrm{I})$ then in the presence of $\mathrm{Ag}(\mathrm{I}) \quad\left(\mathrm{Ag}^{+} \quad \mathrm{V}_{\max }=14.8 \mu \mathrm{mol} / \mathrm{mg} / \mathrm{h} \quad(246.67 \mathrm{nmol} / \mathrm{min} / \mathrm{mg}) ; \mathrm{Cu}^{+}\right.$ $\operatorname{Vmax}=3.7 \mu \mathrm{mol} / \mathrm{mg} / \mathrm{h}(61.67 \mathrm{nmol} / \mathrm{min} / \mathrm{mg}))$, even though it showed 10 times higher affinity for $\mathrm{Cu}^{+}$compared with $\mathrm{Ag}^{+}\left(\mathrm{Ag}^{+} \mathrm{K}_{1 / 2}=29.4 \mu \mathrm{M} ; \mathrm{Cu}^{+} \mathrm{K}_{1 / 2}=2.1 \mu \mathrm{M}\right)$. Ions were present in a form of $100 \mu \mathrm{M} \mathrm{Cu}_{2} \mathrm{SO}_{4}, 2.5 \mathrm{mM}$ DTT, or $100 \mu \mathrm{M} \mathrm{AgNO}$.

For the homolog of CopA from A. fulgidus, Mandal et al in 2002, have shown that the constants values obtained in the presence of silver are higher than in the presence of copper (Mandal \& Argüello, 2003). That discovery goes with the data obtain in this study, since the data obtained here shows the value of E. coli CopA $\mathrm{V}_{\max }$ is higher than for the one obtained for copper in the study of Fan et al in 2002 (Fan \& Rosen, 2002). 


\subsubsection{CopA wild type shows active transport activity towards silver ions}

The idea for the CopA wild type assay in proteoliposomes was to show that observations of the metal ion transport are possible in vitro. The setup of the assay is described in 5.1.1. As shown in Figure 55, CopA wild type proteoliposomes show activity when reconstituted in liposomes. The dye PGSK was incapsulated inside the proteoliposomes and when ATP was added to the proteoliposomes, the active transport of silver ions to the inside of the proteoliposomes is initiated. The observed quenching of PGSK is a result of the metal ions binding to the dye. The control liposomes do not show quenching of PGSK, since there is no enzyme that would transport metal ions inside the liposome. The signal is stable for at least five minutes. Additionally, sodium azide was used as a control, in order to possibly inhibit the activity of CopA. It was shown in previous studies that hydrolysis of ATP by CopA (in detergent micelles) was inhibited by sodium azide (for 80-90 \%) and it was suggested that it is likely that the azide-sensitive ATPase activity is due to minor contamination by a highly active ATPase (Fan \& Rosen, 2002). In the study at hand, sodium azide reduced the activity of CopA, around $20 \%$, which was observed by decrease of fluorescence quenching as compared with CopA wild type proteoliposomes not treated with sodium azide (Figure 56, Figure 57). This minimal reduction of the PGSK-quenching can be expected since sodium azide is not an inhibitor of P-type ATPases. During the experiments it was also visible that azide interacts with some of the compounds, most likely with silver nitrate, forming white precipitate, which interact with the membrane of the (proteo)liposome. The same behaviour is observed also with control liposomes, silver nitrate and azide - PGSK gets quenched since some ions of silver can access it and they can bind to it, by the fact that the membrane is disrupted with the formation of silver azide (Equation 1).

Equation 1. Chemical reaction of the formation of silver azide.

$$
\mathrm{NaN}_{3}+\mathrm{AgNO}_{3} \rightarrow \mathrm{AgN}_{3}+\mathrm{NaNO}_{3}
$$

When there is no silver present in the buffer, and ATP is still added to the solution, some additional quenching was observed, which does not come from the silver ions binding to the 
PGSK but is the background quenching coming from the magnesium ions present in the buffer (5.1.2.3).

For the assay used with CopA, an interesting finding was made. PGSK fluorescence is quenched also with magnesium ions. This new finding was tested when the chelator EDTA is present in the buffer with $\mathrm{Mg}^{2+}$. In that case the fluorescence of the dye is stable for at least five minutes. The same is noticed when no $\mathrm{Mg}^{2+}$ is present in the buffer, or EDTA is present alone in the buffer. However, when $\mathrm{Mg}^{2+}$ is present in the buffer without EDTA, the fluorescence signal decreases, indicating the binding of magnesium ions with the dye (Figure 53). This effect of $\mathrm{Mg}^{2+}$ is important, since $\mathrm{Mg}^{2+}$ ions are present in the buffer used for the CopA assay and are necessary for the activity of ATP. This knowledge helps additionally in explaining the background (quenching of PGSK fluorescence with no substrate) in the CopA wild type proteoliposome assay (5.1.2.5).

\subsubsection{Reconstitution with freeze-thaw procedure}

Even though the reconstitution efficiency showed to be good for the detergent-mediated reconstitution for CopA wild type, the samples were not of such a good quality and the results obtained seemed to be misleading and confusing (data not shown). To overcome this mechanical reconstitution via freeze and thaw cycles was used. The idea was that this method would minimize the possible disruption of the proteoliposome formation during detergent usage. No detergent usage will enable the formation of higher oligomeric states that are perhaps desirable for the studies, or the existing oligomeric state would be more stable if there is no additional detergent in the sample. In the case of CopA (5.1.2.5), as well as in the case of CusA (5.2.2.4), this turned out to be the best way to perform reconstitution, adding apparently to the stability of the formed proteoliposomes. 


\subsection{The tripartite $\mathrm{Cu}(\mathrm{I})$ and $\mathrm{Ag}(\mathrm{I})$ efflux pump CusCBA can sequester metal ions from the periplasm and from the cytoplasm}

\subsubsection{Introduction}

The structures of CusA suggest that this pump is able to take up metals from both the periplasm and the cytoplasm (Long et al., 2010). This ability of the RND pump to transport ions from both, the periplasm and the cytoplasm is postulated due to the usage of a methionine network (methionine cluster), that exports copper or silver ions. The CusA periplasmic cleft constituted by the PC1/PC2 subdomains stays closed in the apo-state, while the cleft is in an open state when a copper(I) or silver(I) ion is bound to the M573/M623/M672 three sulphur-coordination site (Long et al., 2010).

The methionine network in the CusA transmembrane domain consists of three methionine pairs, M410-M501, M486-M403, M391-M1009. With this, CusA can transport ions directly from the cytoplasm across the inner and outer membrane towards the outside of the cell. Another potential way is the transport from the periplasm, where metal ions bind to the threemethionine binding site; another methionine pair is present, M755-M271 and together with M672-M573-M623 represents the periplasmatic pathway of metal ions (Figure 58). Transport from the cytoplasm has been shown by a stop-flow transport assay, where it was observed how CusA transports silver ions from the outside to the inside of the CusA-reconstituted proteoliposomes (Long et al., 2010). 


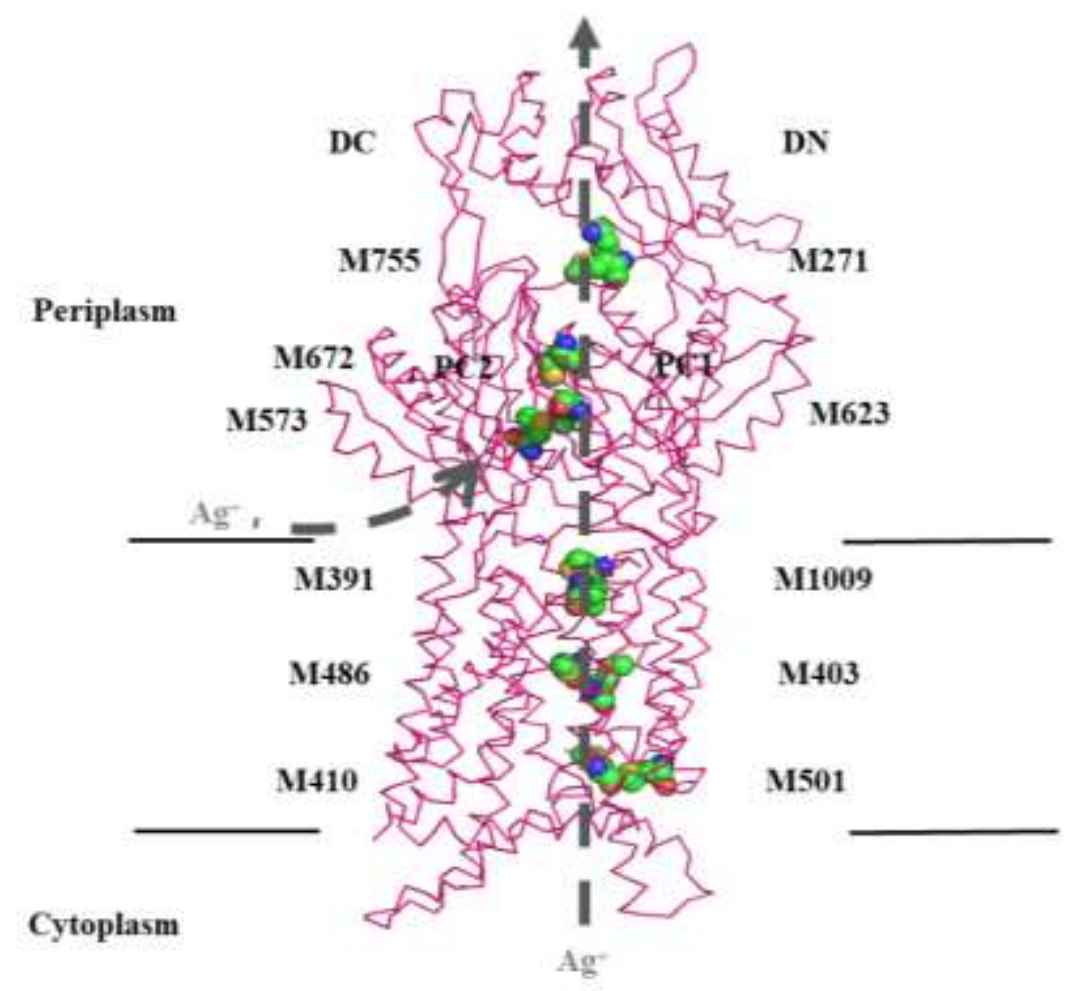

Figure 58. Suggested pathway of silver ions through CusA efflux pump (PDB: 3K07). CusA is found as a trimer in the membrane; here the transport through monomer was shown. Dashed arrows indicate the postulated way of metal ions through the transporter. Ions can be transported either via direct transport from the cytoplasm or via the periplasmic pathway binding to the three-methionine metal-ion-binding site. The five methionine pairs are shown as spheres (C, green; $\mathrm{O}$, red; N, blue; S, yellow). The pair M271-M755 is located at the bottom of the periplasmic funnel domain where the ion could then finally be expelled. The three-methionine binding site is formed by: M672-M573-M623. Additional methionine-pairs in the transmembrane region are formed by: M410-M501, M486-M403 and M391-M1009. Figure was prepared with Pymol (https://pymol.org/). Adapted from (Long et al., 2010).

\subsubsection{Results}

\subsubsection{Production of CusA wild type for reconstitution and transport assays}

CusA wild type was used in this study, and for the purpose of production, it was amplified from the E. coli genome and cloned into the p7XC3H vector and expressed in E. coli C43(DE3) $\triangle a c r A B D$ using IPTG as an inducer. CusA wild type was purified via $\mathrm{Ni}^{2+}$-NTA affinity chromatography and via SEC. In Figure 59, the SEC profile of the purified protein, as well as the purity levels and possible degradations present in the sample are shown by Coomassie-stained SDS-PAGE and Western blot analysis. A single band observed on Figure 
59A and Figure 59B for the purified protein sample corresponded to the expected molecular weight of CusA with His-tag $(115 \mathrm{kDa})$, and a peak at $16.4 \mathrm{ml}$ on the SEC profile was detected (Figure 59C).
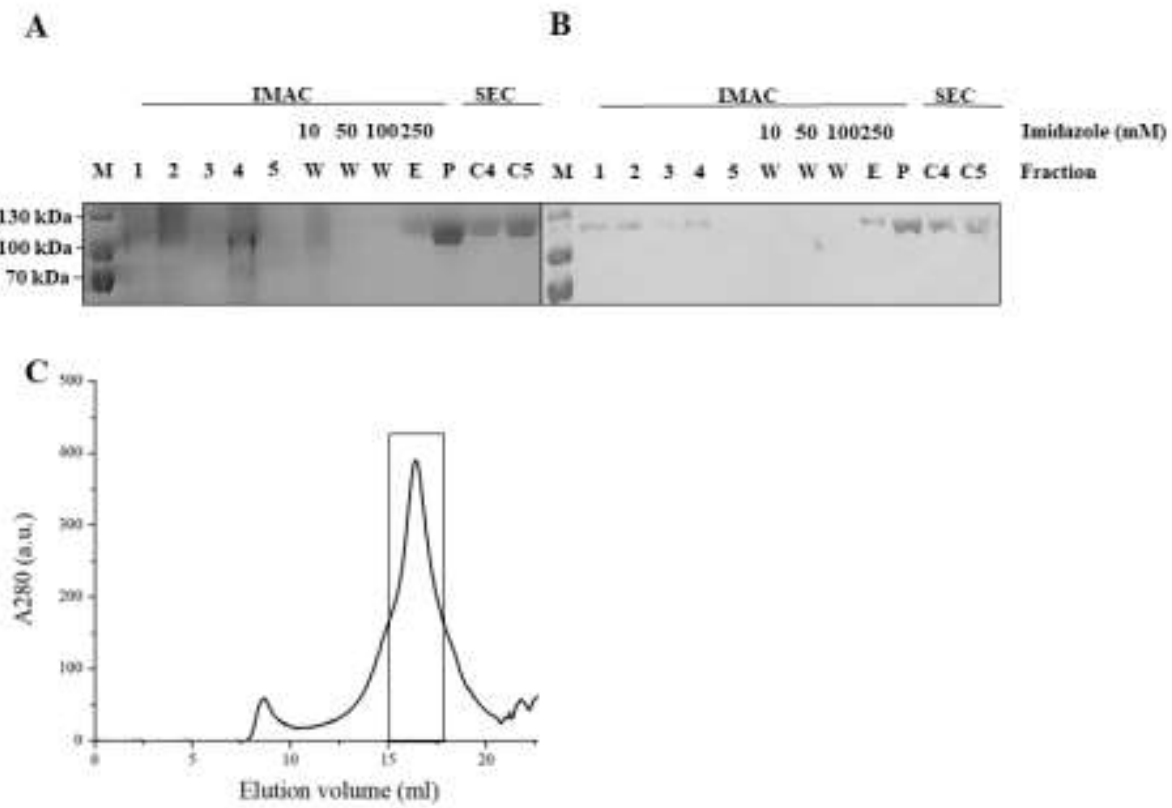

Figure 59. Purification of CusA wild type. A. Coomassie-stained $10 \%$ SDS-PAA gel of purified CusA wild type. B. Western Blot analysis with identical samples from A. The Western Blot was probed with monoclonal anti-polyHistidinealkaline phosphatase produced in mouse (Sigma A5588). M-Marker (PageRuler Prestained Protein Ladder (ThermoScientific)), 1-Membrane, 2-Solubilized membrane, 3-Supernatant, 4-Pellet, 5-Flow through, W-Samples from indicated wash steps from $\mathrm{Ni}^{2+}$-NTA purification, E-Eluate, P-Concentrated protein sample after $\mathrm{Ni}^{2+}$-NTA purification, C4/C5-Samples from the fractions collected after SEC. Applied: 1-E (samples were diluted 12.5x in SDS-sample buffer) $20 \mu \mathrm{l}$ for Coomassie, $5 \mu \mathrm{l}$ for Western blot; P- $5 \mu \mathrm{g}$ for Coomassie, $1 \mu \mathrm{g}$ for Western blot; C4/C5- $3 \mu \mathrm{g}$ for Coomassie, 0.8 $\mu \mathrm{g}$ for Western blot. C. Size exclusion chromatography profile of a $\mathrm{Ni}^{2+}$-NTA purified sample of CusA wild type. The protein was purified on a Superose 6 Increase 30/100 column equilibrated with $20 \mathrm{mM}$ HEPES/NaOH pH 7.6, $200 \mathrm{mM} \mathrm{KCl}$, $0.05 \% \mathrm{DDM}$, with a flow rate of $0.4 \mathrm{ml} / \mathrm{min}$. The peak was detected at $16.4 \mathrm{ml}$.

\subsubsection{Production of CusA D405N variant for reconstitution and transport assays}

CusA D405N is an inactive variant of CusA. D405 is suggested to be involved in proton binding and release, which is pivotal to induce conformational changes which lead to metalion efflux (Long et al., 2010)(Klenotic et al., 2020). To introduce the mutation encoding N405, inverse PCR was performed on cusA (3.2.1.10). The gene containing the mutation was then cloned into the $\mathrm{p} 7 \mathrm{XC} 3 \mathrm{H}$ vector and expressed in $E$. coli $\mathrm{C} 43(\mathrm{DE} 3) \triangle a c r A B D$ using IPTG as an inducer. 
A

B
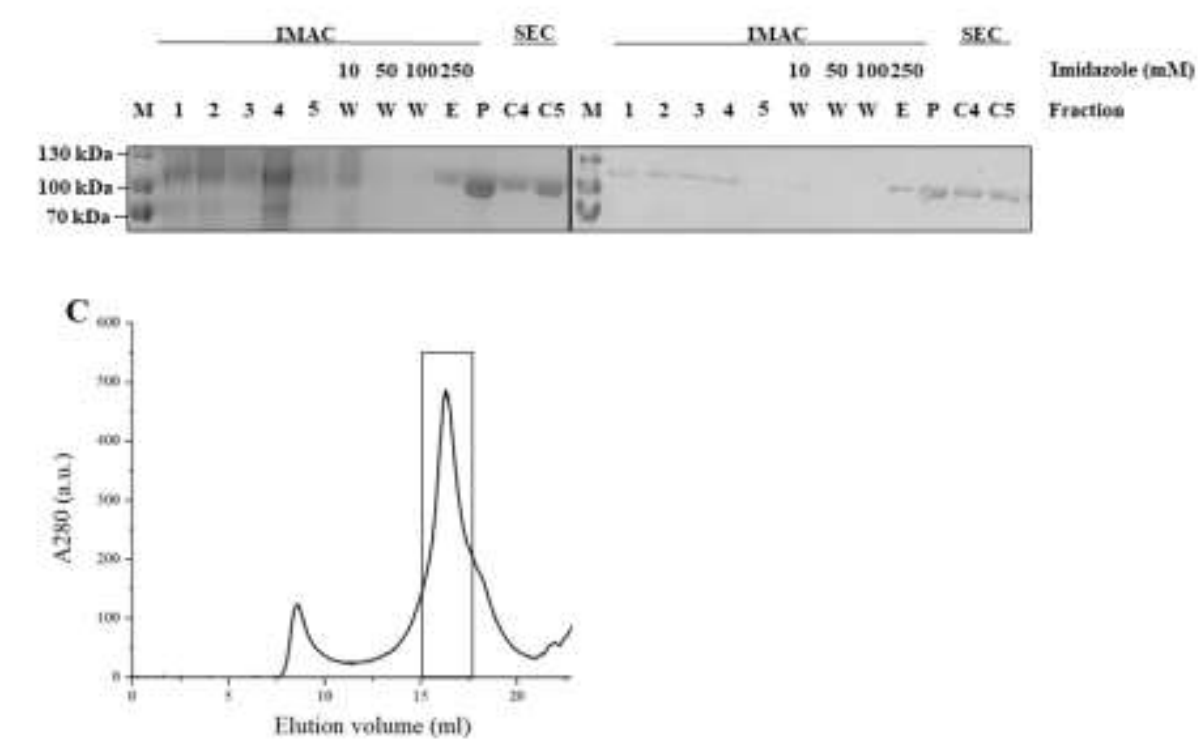

Figure 60. Purification of CusA D405N. A. Coomassie-stained 10\% SDS-PAA gel of purified CusA D405N. B. Western Blot analysis with identical samples from A. The Western Blot was probed with monoclonal anti-polyHistidine-alkaline phosphatase produced in mouse (Sigma A5588). M-Marker (PageRuler Prestained Protein Ladder (ThermoScientific)), 1Membrane, 2-Solubilized membrane, 3-Supernatant, 4-Pellet, 5-Flow through, W-Samples from indicated wash steps from $\mathrm{Ni}^{2+}$-NTA purification, E-Eluate, P-Concentrated protein sample after $\mathrm{Ni}^{2+}$-NTA purification, C4/C5-Samples from the fractions collected after SEC. Applied: 1-E (samples were diluted 12.5x in SDS-sample buffer) $20 \mu \mathrm{l}$ for Coomassie, $5 \mu \mathrm{l}$ for Western blot; P- $5 \mu \mathrm{g}$ for Coomassie, $1 \mu \mathrm{g}$ for Western blot; C4/C5- $3 \mu \mathrm{g}$ for Coomassie, $1 \mu \mathrm{g}$ for Western blot. C. Size exclusion chromatography profile of a $\mathrm{Ni}^{2+}$-NTA purified sample of CusA D405N. The protein was purified on a Superose 6 Increase 30/100 column equilibrated with $20 \mathrm{mM}$ HEPES/NaOH pH 7.6, $200 \mathrm{mM} \mathrm{KCl}, 0.05 \%$ DDM, with a flow rate of $0.4 \mathrm{ml} / \mathrm{min}$. The peak was detected at $16.3 \mathrm{ml}$.

The protein was purified via $\mathrm{Ni}^{2+}$-NTA affinity chromatography and SEC. In Figure 60, SDSPAGE, Western Blot and SEC analysis of the CusA D405N purification are summarized. A single band observed on Figure 60A and Figure 60B for the purified protein sample corresponds to the expected molecular weight of CusA with His-tag (115 kDa), and a peak at $16.3 \mathrm{ml}$ on the SEC profile was detected (Figure 60C).

\subsubsection{Solubilization assay to validate the reconstitution of CusA wild type into artificial membranes}

The solubilization assay was done as described in 3.2.2.19. Figure 61 shows the results of the solubilization assay for the CusA wild type proteoliposomes. The assay was performed for the proteoliposomes prepared for the ACMA assay as well as for the PGSK assay, 
respectively. Two different proteoliposomal samples were made, because during reconstitution for the PGSK assay the dye was added together with the protein, after which freeze thaw cycles were performed. This is different than the preparation for the ACMA assay, where the dye was added directly before the measurement.

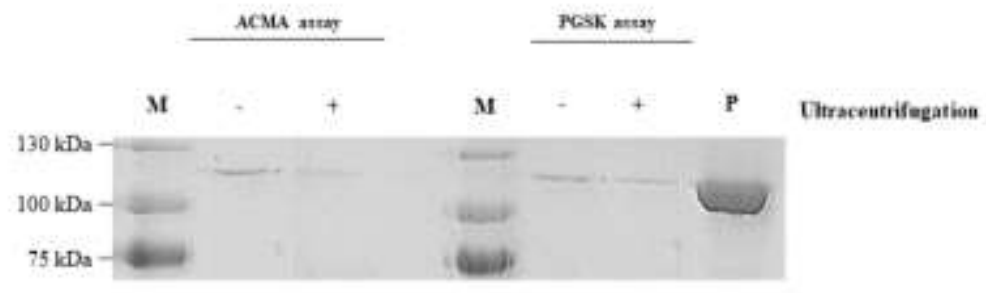

Figure 61. Solubilization assay of CusA wild type. Coomassie-stained $10 \%$ SDS-PAA gel of samples obtained during solubilization assay. The protein was reconstituted for two different assays, where two different dyes were used. When ACMA dye was used as a reporter system, the dye was added directly before the measurements start, while in the PGSK assay, the dye is added during the reconstitution process. M-Marker (PageRuler Prestained Protein Ladder (ThermoScientific)). (-/+)- $15 \mu \mathrm{l}$ samples before and after ultracentrifugation, respectively. For the calculation of the protein amount in liposomes, the sample of the purified protein $(\mathrm{P})$ was loaded on the SDS-PAA gel, P- $6.4 \mu \mathrm{g}$.

Table 20. Protein amounts in the CusA wild type proteoliposomes. Image J (Schneider et al., 2012) was used to analyse SDS-PAA gel bands (Figure 61) to derive protein amounts. Protein amount used in the activity assay (100 $\mu$ l of the sample used in each activity assay) is shown.

\begin{tabular}{|l|l|l|}
\hline $\begin{array}{l}\text { Assay } \\
\text { type }\end{array}$ & $\begin{array}{l}\text { Reconstitution efficiency } \\
(\boldsymbol{\%})\end{array}$ & $\begin{array}{l}\text { Protein amount used in the } \\
\text { activity assay }(\boldsymbol{\mu g})\end{array}$ \\
\hline ACMA & 13 & 1.5 \\
\hline PGSK & 14 & 1.6 \\
\hline
\end{tabular}

If the bands before (-) and after (+) ultracentrifugation have the same intensities, $100 \%$ of protein is successfully incorporated in the liposomes. The band's intensities before and after ultracentrifugation are compared with a sample of purified protein of known concentration $(6.4 \mu \mathrm{g})$. The comparison was done using ImageJ (Schneider et al., 2012). For the preparation with ACMA, $36 \%$ of protein is incorporated correctly, while for the preparation with PGSK - $57 \%$. As mentioned for the solubilization assays of AcrB proteoliposomes, the results can vary batch to batch and show the quantitative but not the qualitative side of the samples. The reconstitution efficiency was calculated as well, as a percentage of protein incorporated in liposomes compared to the amount of protein added in the beginning of reconstitution (Table 20, Table 19). Table 20 shows the protein amounts for the preparation of the ACMA and PGSK assay. 


\subsubsection{Different orientations of CusA in liposomes can be triggered by the application of different conditions on the outside of the proteoliposomes}

Upon reconstitution, CusA can adopt different orientations. If the transporter is oriented inside-out, meaning with the periplasmic domain located inside the liposome, transport equivalent to cytoplasmatic transport can be observed (Long et al., 2010). To trigger the $\mathrm{H}^{+} / \mathrm{Ag}^{+}$antiport, the buffer inside the liposome should be acidic, that on the outside of the proteoliposomes alkaline and should contain the transported ions. The reporter system in this case is Phen Green SK (PGSK; $\lambda_{\mathrm{ex}}=480 \mathrm{~nm} ; \lambda_{\mathrm{em}}=530 \mathrm{~nm}$ ), its fluorescence is quenched upon binding of metal ions entering the inside of the proteoliposomes (Figure 62A).

A)

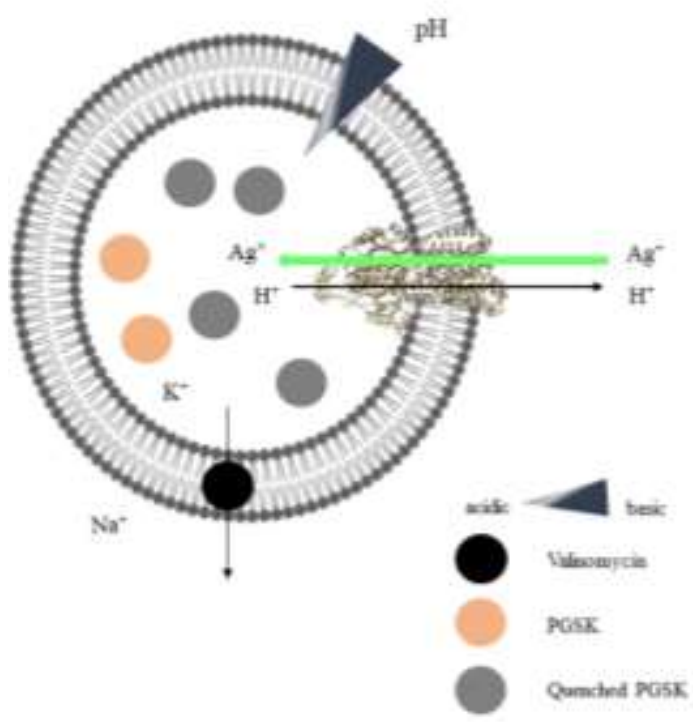

B)

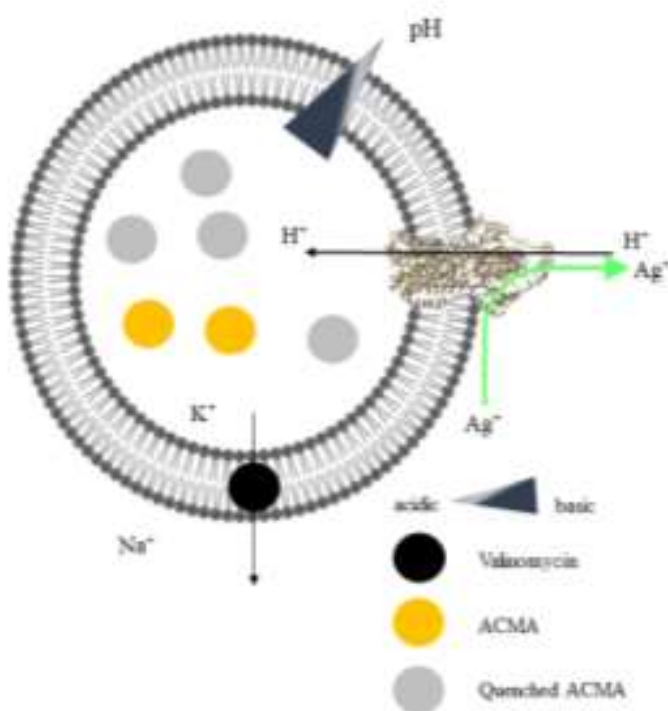

Figure 62. $\mathrm{Ag}^{+} / \mathrm{H}^{+}$antiport of the CusA efflux pump in the two expected different orientations of RND pump after reconstitution into liposomes. Transport can be triggered by imposing a $\Delta \mathrm{pH}$ across the proteoliposomal membrane. A. Inside-out orientation of CusA; transport of $\mathrm{Ag}^{+}$to the inside of the proteoliposomes can be monitored with the $\mathrm{Ag}^{+}-$ reporting dye - Phen Green SK (PGSK, pink circle) - $\lambda_{\mathrm{ex}}=480 \mathrm{~nm} ; \lambda_{\mathrm{em}}=530 \mathrm{~nm}$ if the $\Delta \mathrm{pH}$ is imposed by acidic buffer inside and alkaline buffer outside. B. Right-side out orientation; in this case the $\mathrm{Ag}^{+}$ions are expected to follow the periplasmic route for $\mathrm{Ag}^{+}$transport. Antiport can be monitored by the influx of protons from the outside toward the inside with the $\mathrm{pH}$-sensitive dye- 9-Amino-6-chloro-2-methoxyacridine (ACMA, yellow circle)- $\lambda_{\mathrm{ex}}=410 \mathrm{~nm}$; $\lambda_{\mathrm{em}}=500 \mathrm{~nm}$ ). Quenched PGSK and ACMA, are represented with dark grey and light grey circles, respectively. For some experiments, valinomycin (black circle) was used for the generation of an electrochemical gradient over the membrane.

If the transporter is oriented right-side out, with the periplasmic domain oriented outside the liposome, periplasmic $\mathrm{Ag}^{+}$transport can be anticipated. The buffer outside the 
proteoliposomes should be acidic and inside alkaline. $\mathrm{Ag}^{+}$added to the outside would trigger the periplasmic binding and release of $\mathrm{Ag}^{+}$coupled to the influx of protons. The latter transport can be monitored via the dye $\operatorname{ACMA}\left(\lambda_{\mathrm{ex}}=410 \mathrm{~nm} ; \lambda_{\mathrm{em}}=500 \mathrm{~nm}\right)$ (Figure 62A, Figure 62B). When the transporter is active, metal ions would be circulating in the extra liposomal space, transported via the periplasmic pathway through CusA, while protons would be transported inside the liposome (substrate-proton antiport).

CusA wild type was reconstituted into the lipid bilayer via freeze/thaw cycles, where the mechanical force during freeze and thaw cycles incorporates the membrane protein into the liposomal membrane. To obtain even sized proteoliposomes, extrusion through a filter membrane was performed two times: before freeze/thaw cycles and after performing them (which is done upon incubation of protein with solubilized lipid solution). Valinomycin $(50 \mathrm{nM})$ was added to the (proteo)liposomes just before the initiation of the assay.

\subsubsection{ACMA as a reporter system of the right-side out oriented CusA in liposomes}

Initially, the experiments were done with a $\mathrm{pH}$ gradient only. The buffer inside the (proteo)liposomes contained the same salt composition as the buffer in which the liposomes were diluted, and valinomycin was added to the system. CusA proteoliposomes were first prepared in buffer containing $20 \mathrm{mM}$ HEPES/KOH pH 7.6 or $\mathrm{pH} 6.6,50 \mathrm{mM} \mathrm{K} \mathrm{SO}_{4}$. These were then diluted into the same buffer at $\mathrm{pH} 7.6$ or $\mathrm{pH} 6.6(\Delta \mathrm{pH}$ alkaline outside), in the presence of $500 \mu \mathrm{M} \mathrm{AgNO}$. In the experiments, the $\mathrm{H}^{+}$-transport was recorded by the changes of ACMA fluorescence. A slight quenching is being observed under conditions where a $\Delta \mathrm{pH}$ was applied (magenta curve, Figure 63), whereas without a gradient, a slow but linear quenching of the ACMA signal was observed over time (blue curve, Figure 63). When the $\Delta \mathrm{pH}$ was inverted by preparing the CusA proteoliposomes in $20 \mathrm{mM} H E P E S / K O H ~ p H$ 7.6, $50 \mathrm{mM} \mathrm{K} \mathrm{SO}_{4}$ and diluted into $20 \mathrm{mM}$ HEPES/KOH pH $6.6(\Delta \mathrm{pH}$ inside acidic) or $\mathrm{pH}$ 7.6 (no gradient), including $50 \mathrm{mM} \mathrm{K} \mathrm{SO}_{4}$ and $500 \mu \mathrm{M} \mathrm{AgNO} 3$, a more prominent and sustainable quenching of the dye ACMA was observed with a $\Delta \mathrm{pH}$ applied (orange curve, Figure 63) compared to when no $\mathrm{pH}$ gradient was imposed (black curve, Figure 63). 


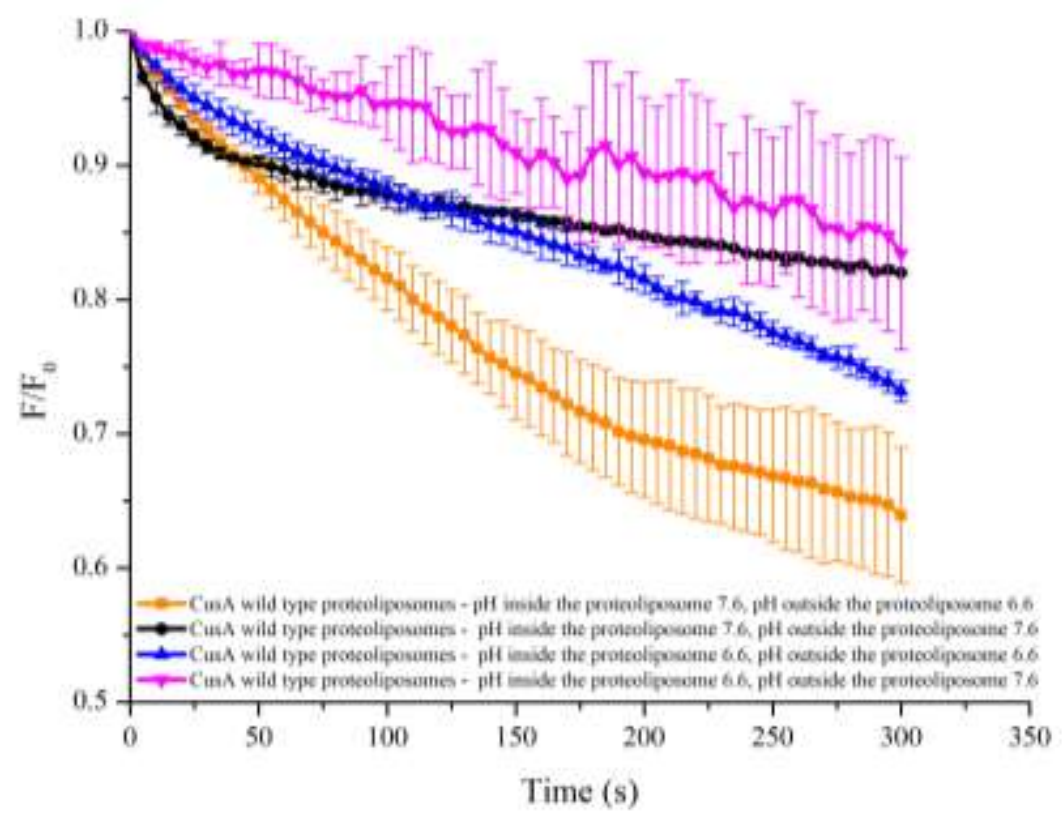

Figure 63. CusA-mediated ACMA quenching. Different buffers were used inside and outside the liposomes. Buffer inside the liposome: $20 \mathrm{mM}$ HEPES/KOH pH 7.6/6.6, $50 \mathrm{mM} \mathrm{K}_{2} \mathrm{SO}_{4}$. Buffer outside the liposome: $20 \mathrm{mM} \mathrm{HEPES} / \mathrm{KOH} \mathrm{pH}$ $6.6 / 7.6,50 \mathrm{mM} \mathrm{K}_{2} \mathrm{SO}_{4}, 500 \mu \mathrm{M} \mathrm{AgNO}_{3}$. Fluorescence of ACMA was monitored as a function of time $\left(\lambda_{\mathrm{ex}}=410 \mathrm{~nm} ; \lambda_{\mathrm{em}}=\right.$ $500 \mathrm{~nm}$ ). Fluorescence was normalized to fold change, $\mathrm{F} / \mathrm{F}_{0}$ (division by the initial value). Data points are average of: three consecutive measurements $(\mathrm{n}=3 ; \mathrm{pH}$ inside $7.6, \mathrm{pH}$ outside $6.6 / \mathrm{pH}$ inside $6.6, \mathrm{pH}$ outside 6.6 ), two consecutive measurements $(\mathrm{n}=2 ; \mathrm{pH}$ inside $7.6, \mathrm{pH}$ outside $7.6 / \mathrm{pH}$ inside $6.6, \mathrm{pH}$ outside 7.6).

The same experiment was done with additional control, with liposomes that do not have incorporated protein in the lipid bilayer.

When buffer inside the proteoliposomes is $\mathrm{pH} 6.6$ and those proteoliposomes are diluted into the buffer $\mathrm{pH} 7.6$, the signal of the fluorescence is similar to the control liposomes (magenta and blue curve, respectively, Figure 64). When the $\mathrm{pH}$ inside the vesicle is 7.6 and outside the vesicle 6.6, a clear quenching of the ACMA dye for CusA wild type proteoliposomes in comparison to the control liposomes (no protein) (orange and black curve, respectively, Figure 65) can be observed. This set up gave a first insight of the events happening in the proteoliposome and information about the activity of CusA (Figure 65). The black and orange curves in Figure 64 and Figure 65, respectively, and the orange curve only in Figure 63, Figure 64, and Figure 65, respectively, represent identical experiments. 


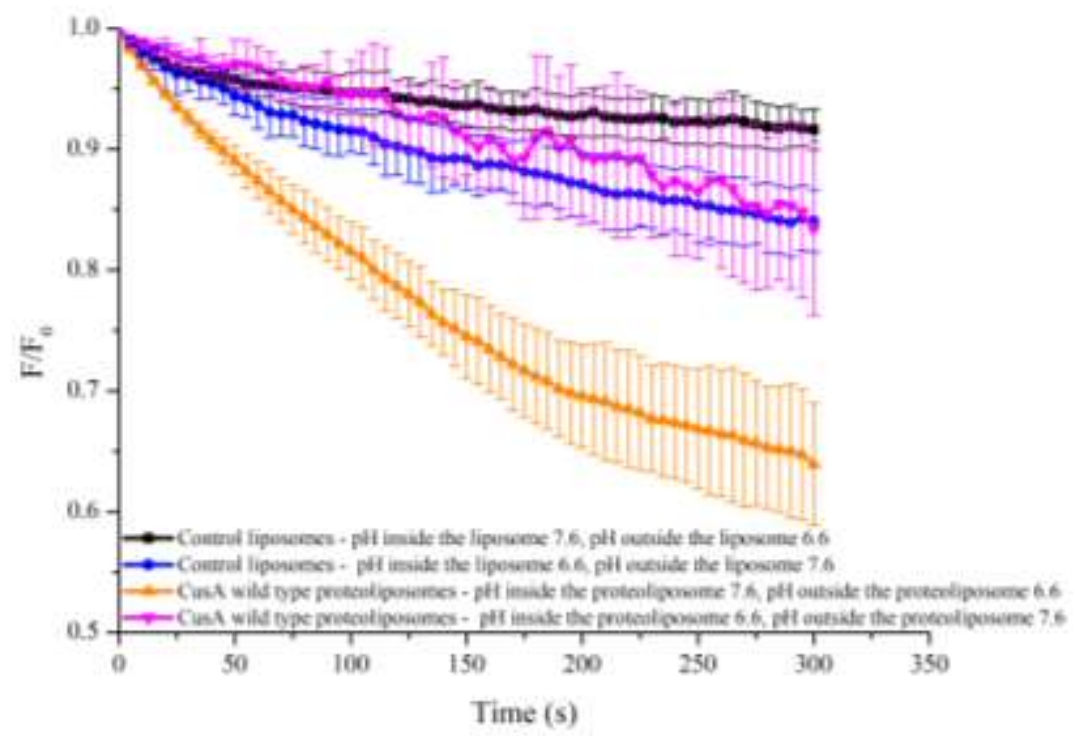

Figure 64. CusA-mediated ACMA quenching. CusA wild type proteoliposomes and control liposomes subjected to the different buffer conditions. Buffer inside the liposome: $20 \mathrm{mM}$ HEPES/KOH pH 7.6/6.6, $50 \mathrm{mM} \mathrm{K}_{2} \mathrm{SO}_{4}$. Buffer outside the liposome: $20 \mathrm{mM}$ HEPES/KOH pH 6.6/7.6, $50 \mathrm{mM} \mathrm{K}_{2} \mathrm{SO}_{4}, 500 \mu \mathrm{M} \mathrm{AgNO}$. Fluorescence of ACMA dye was monitored as a function of time $\left(\lambda_{\mathrm{ex}}=410 \mathrm{~nm} ; \lambda_{\mathrm{em}}=500 \mathrm{~nm}\right.$ ). Fluorescence was normalized to fold change, $\mathrm{F} / \mathrm{F}_{0}$ (division by the initial value). Data points are average: three consecutive measurements $(\mathrm{n}=3$; CusA wild type proteoliposomes - $\mathrm{pH}$ inside 7.6, $\mathrm{pH}$ outside 6.6/Control liposomes - pH inside 7.6, pH outside 6.6/ Control liposomes - pH inside 6.6, $\mathrm{pH}$ outside 7.6), two consecutive measurements ( $\mathrm{n}=2$; CusA wild type proteoliposomes - $\mathrm{pH}$ inside $6.6, \mathrm{pH}$ outside 7.6). The curves with CusA proteoliposomes are from the same experiment shown in Figure 63.

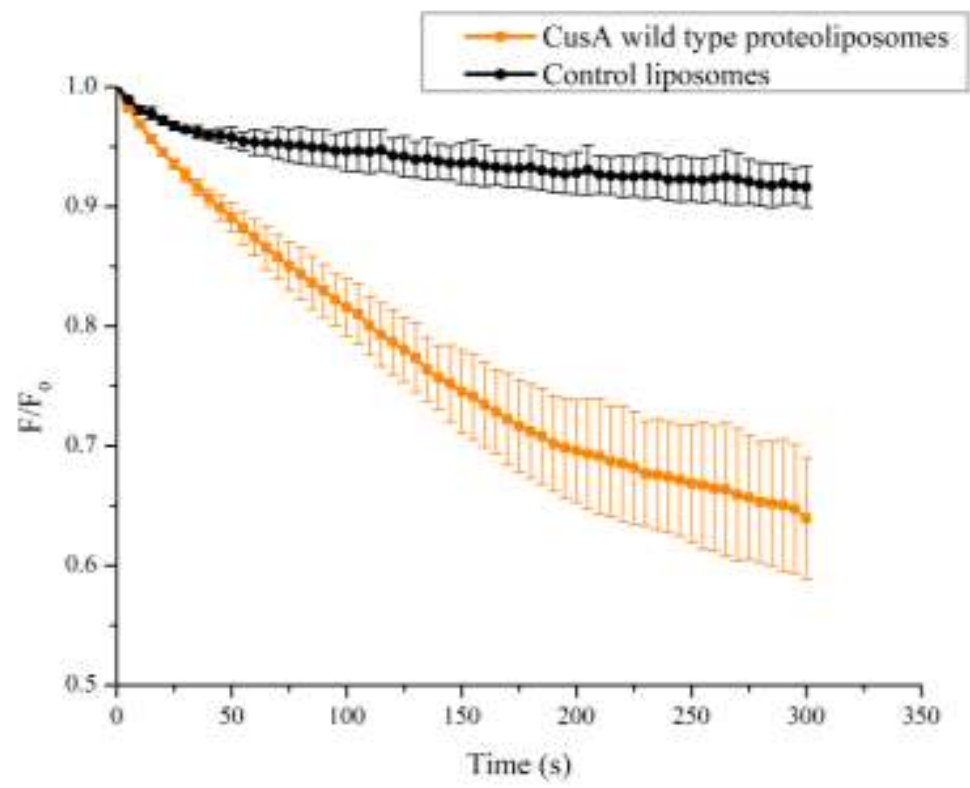

Figure 65. CusA wild type shows activity when reconstituted into the liposomes. Empty liposomes are shown as a control. Buffer inside the liposome: $20 \mathrm{mM}$ HEPES/KOH pH 7.6, $50 \mathrm{mM} \mathrm{K}_{2} \mathrm{SO}_{4}$. Buffer outside the liposome: $20 \mathrm{mM} \mathrm{HEPES} / \mathrm{KOH}$ $\mathrm{pH}$ 6.6, $50 \mathrm{mM} \mathrm{K}_{2} \mathrm{SO}_{4}, 500 \mu \mathrm{M} \mathrm{AgNO}_{3}$. Driving force: $\Delta \mathrm{pH}$. Fluorescence of ACMA dye was monitored as a function of time $\left(\lambda_{\mathrm{ex}}=410 \mathrm{~nm} ; \lambda_{\mathrm{em}}=500 \mathrm{~nm}\right)$. Fluorescence was normalized to fold change, $\mathrm{F} / \mathrm{F}_{0}$ (division by the initial value). Data points are average of three consecutive measurements $(n=3)$. 


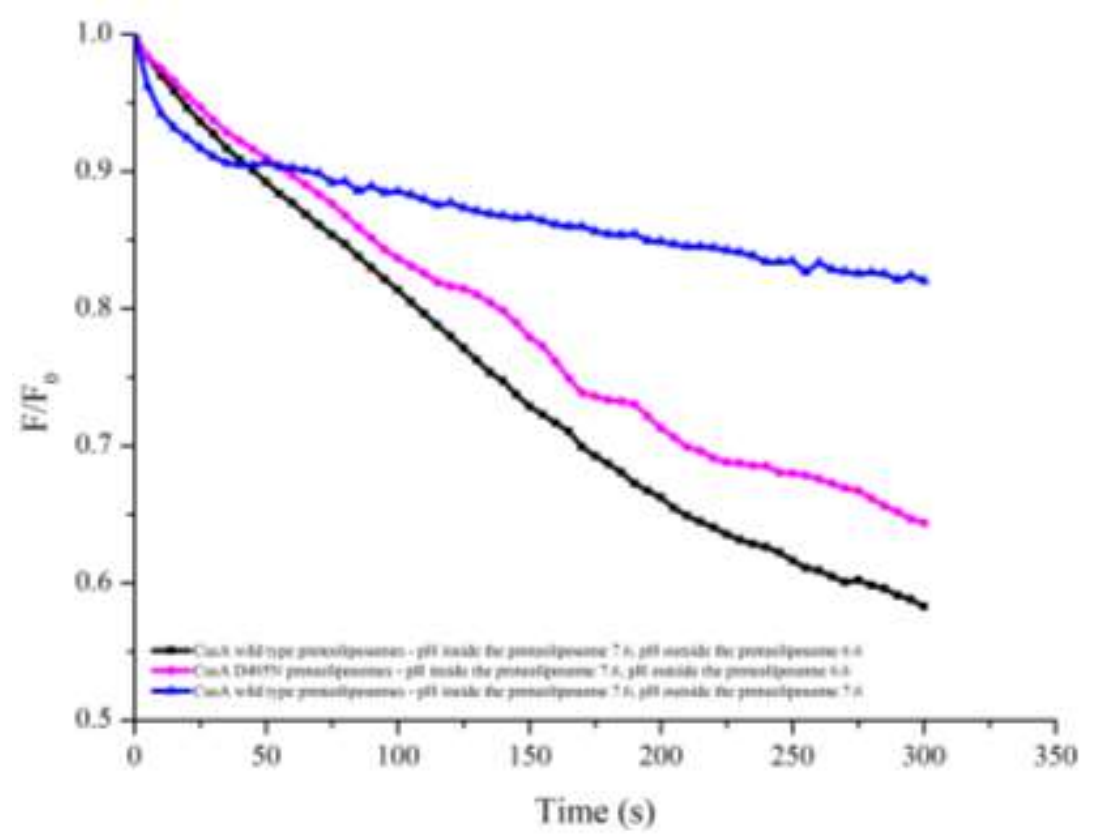

Figure 66. CusA-mediated ACMA quenching. CusA wild type and CusA D405N proteoliposomes in different buffer conditions. Buffer inside the liposome: $20 \mathrm{mM}$ HEPES/KOH pH 7.6, $50 \mathrm{mM} \mathrm{K}_{2} \mathrm{SO}_{4}$. Buffers outside the liposome: $20 \mathrm{mM}$ $\mathrm{HEPES} / \mathrm{KOH} \mathrm{pH} 6.6 / 7.6,50 \mathrm{mM} \mathrm{K} \mathrm{SO}_{4}, 500 \mu \mathrm{M} \mathrm{AgNO}_{3}$. Fluorescence of ACMA dye was monitored as a function of time $\left(\lambda_{\mathrm{ex}}=410 \mathrm{~nm} ; \lambda_{\mathrm{em}}=500 \mathrm{~nm}\right)$. Fluorescence was normalized to fold change, $\mathrm{F} / \mathrm{F}_{0}$ (division by the initial value).

For additional control, variant of CusA, D405N, was used. This variant carries the mutation in the proton relay network, at D405 position, and it has been shown to be inactive (Long et $a l ., 2010)$. When reconstituted in the liposome, CusA D405N seems not to lose the activity completely, as detected by ACMA quenching (protons are being transported inside the proteoliposome) (Figure 66, magenta curve). However, the quenching is not as prominent as for the CusA wild type (Figure 66, black curve). For the comparison of the non-transport, CusA wild type proteoliposomes are shown, where no $\Delta \mathrm{pH}$ was imposed (Figure 66, blue curve), where only limited quenching was observed (blue curve).

When a $\mathrm{pH}$ gradient was imposed over the membrane, $\mathrm{H}^{+}$-influx was observed for both CusA wild type and (to a lesser extent) the D405N variant Figure 66. Using the same conditions and the same direction and extent of $\Delta \mathrm{pH}$ with control liposomes i.e., with no CusA or CusA D405N present, almost no change in the fluorescent signal was observed (Figure 65, black curve).

Since reconstitution yields both inside-out and right-side out orientation of CusA into the liposomes, different buffer compositions were used to trigger transport for the differently oriented CusA molecules in the proteoliposomes. To investigate the influence of $\Delta \psi$, the 
buffer compositions were modified to include sodium. For transport via CusA in the rightside out orientation, the buffer inside the proteoliposomes (buffer 2 on Figure 67) is $20 \mathrm{mM}$ HEPES pH 7.6/KOH, $50 \mathrm{mM} \mathrm{K} 2 \mathrm{SO}_{4}$, while buffers in which the (proteo)liposomes are diluted $(1: 10, \mathrm{v} / \mathrm{v})$ were either the same buffer 2 (reconstitution buffer), or buffer $1-20 \mathrm{mM}$ HEPES/NaOH pH 6.6, $50 \mathrm{mM} \mathrm{Na} \mathrm{SO}_{4}, 1 \mathrm{mM} \mathrm{AgNO} 3$ or buffer 3 - $20 \mathrm{mM}$ HEPES/NaOH $\mathrm{pH}$ 7.6, $50 \mathrm{mM} \mathrm{Na}_{2} \mathrm{SO}_{4}, 1 \mathrm{mM} \mathrm{AgNO}$. Different buffers are imposing different gradients or no gradient (in case of dilution into the same buffer as inside the proteoliposomes). Transport of $\mathrm{H}^{+}$was detected by monitoring the changes in the fluorescent signal of the dye ACMA over time. The quenching of the fluorescent signal of the dye indicates proton influx into the proteoliposomes (5.2.2.4). When proteoliposomes are being diluted into buffer 2, which is the same buffer that is inside the proteoliposomes, only a minimal amount of quenching is observed (green curve, Figure 67). By dilution into buffer 3, a $\Delta \mathrm{pNa}^{+}$(directed towards the inside) and a $\Delta \psi$ (negative inside) is imposed, resulted into a largest quenching of the ACMA fluorescence over time (blue curve, Figure 67). When a $\Delta \mathrm{pH}$, a $\Delta \mathrm{pNa}^{+}$and a $\Delta \psi$ (negative inside) was imposed (dilution into buffer 1), the strong quenching was observed as well (orange curve, Figure 67). That indicates that gradients applied over the membrane, either chemical or electrical or both, result in the transport of $\mathrm{H}^{+}$across the membrane. 


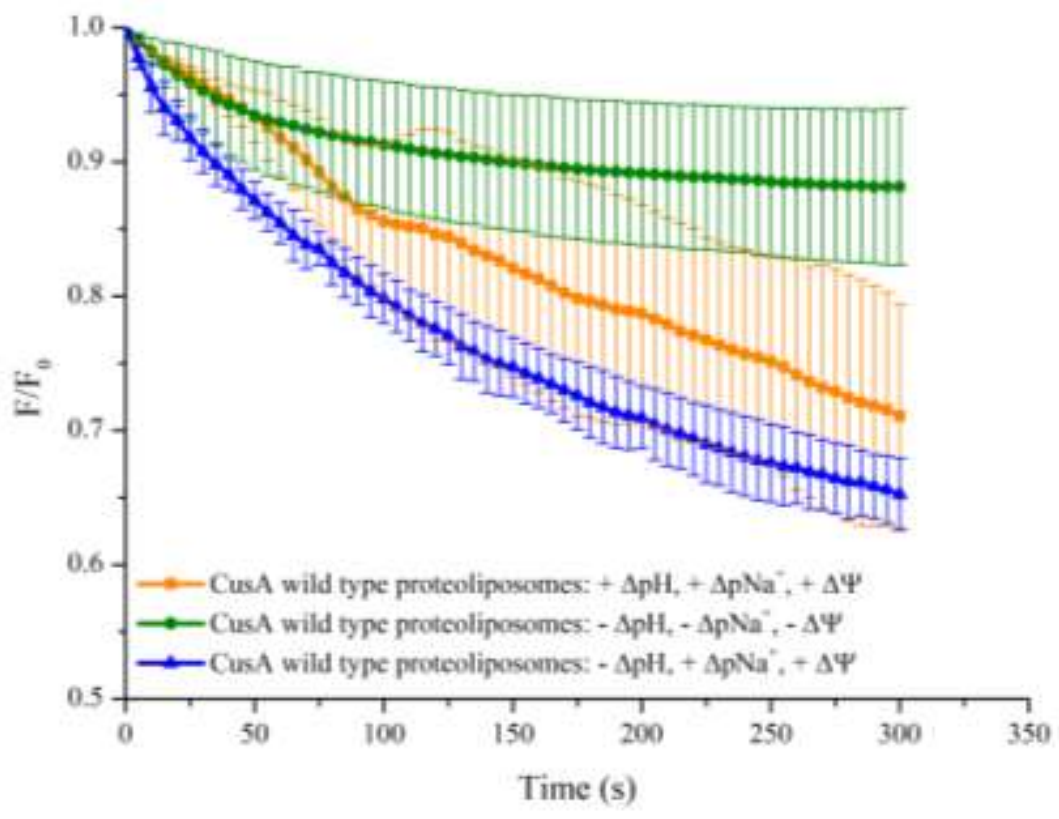

Figure 67. CusA-mediated ACMA quenching. CusA wild type proteoliposomes were subjected to different buffer solutions, imposing (+) or not imposing (-) different driving forces $\left(\Delta \mathrm{pH}\right.$, a $\Delta \mathrm{pNa}^{+}$and a $\left.\Delta \psi\right)$. Buffer 1: $20 \mathrm{mM}$ HEPES/NaOH pH 6.6, $50 \mathrm{mM} \mathrm{Na} 2 \mathrm{SO}_{4}, 1 \mathrm{mM} \mathrm{AgNO}$ (orange curve); Buffer 2: $20 \mathrm{mM}$ HEPES pH 7.6/KOH, $50 \mathrm{mM} \mathrm{K}_{2} \mathrm{SO}_{4}$ (green curve); Buffer 3: $20 \mathrm{mM}$ HEPES/NaOH pH 7.6, $50 \mathrm{mM} \mathrm{Na}_{2} \mathrm{SO}_{4}, 1 \mathrm{mM} \mathrm{AgNO}_{3}$ (blue curve). CusA wild type proteoliposomes were prepared in buffer: $20 \mathrm{mM}$ HEPES $\mathrm{pH} 7.6 / \mathrm{KOH}, 50 \mathrm{mM} \mathrm{K} \mathrm{SO}_{4}$. Fluorescence of ACMA was monitored as a function of time $\left(\lambda_{\mathrm{ex}}=410 \mathrm{~nm} ; \lambda_{\mathrm{em}}=500 \mathrm{~nm}\right)$. Fluorescence was normalized to fold change, $\mathrm{F} / \mathrm{F}_{0}$ (division by the initial value). Data points are average of two consecutive measurements $(n=2)$.

However, it is necessary to look at the additional controls in order to be sure that this observed $\mathrm{H}^{+}$-transport is CusA-mediated and not due to non-protein-mediated diffusion of $\mathrm{H}^{+}$ into the liposomes. For that purpose, empty liposomes (control liposomes, Figure 68), were prepared.

When buffer 2 was used (no gradient applied), the control liposomes are behaving as expected, no additional quenching is observed (magenta curve, Figure 68). When an electrochemical gradient (dilution into buffer 1, a $\Delta \mathrm{pNa}^{+}$(directed towards the inside), a $\Delta \psi$ (negative inside) and a $\Delta \mathrm{pH}$ (acidic outside)) is applied across the membrane of the liposome, minor quenching is detected over time, which can indicate slight $\mathrm{H}^{+}$leakage in these liposomes (black curve, Figure 68). The amount of quenching was comparable to the quenching seen in the assay with CusA-proteoliposomes in the absence of any gradient (black curve, Figure 68). The CusA-mediated transport under conditions where $\Delta \mathrm{pNa}, \Delta \psi$ (negative inside) and a $\Delta \mathrm{pH}$ were imposed led in comparison to a more sustainable quenching over time (orange curve, Figure 68). Orange and green curves in Figure 67 and Figure 68, represent identical experiments. 


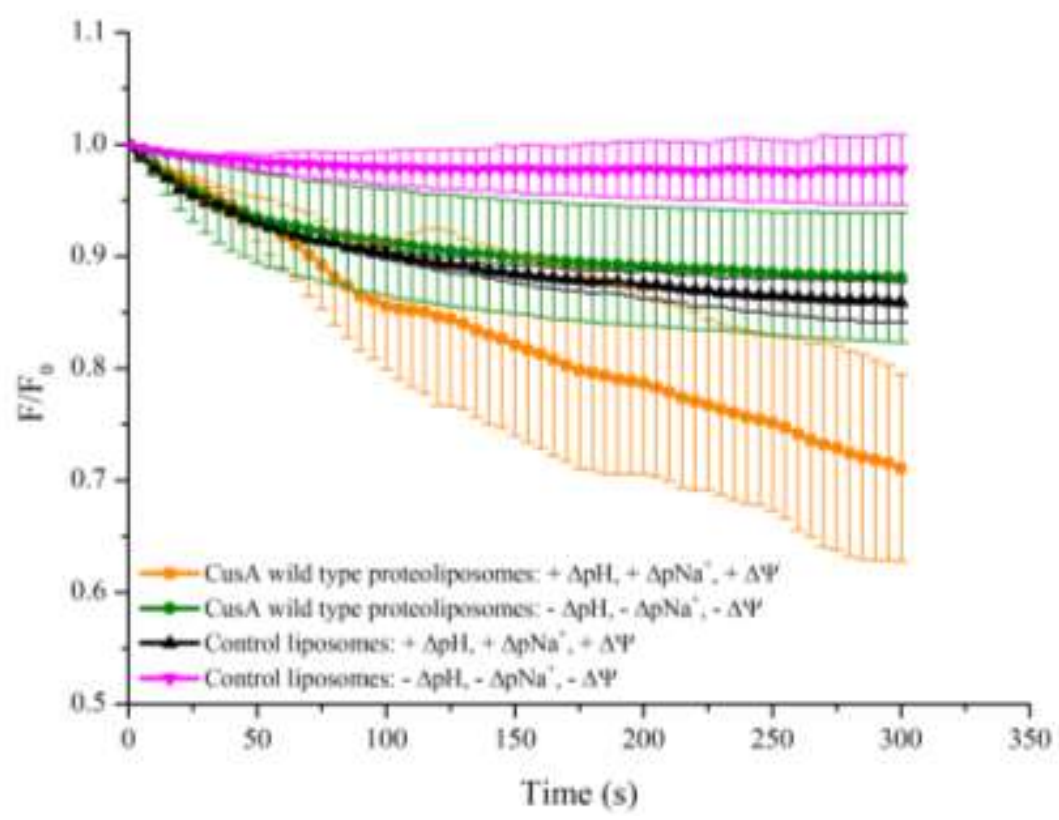

Figure 68. CusA-mediated ACMA quenching. CusA wild type proteoliposomes and control liposomes were subjected to different buffer solutions, imposing (+) or not imposing (-) different driving forces $\left(\Delta \mathrm{pH}\right.$, a $\Delta \mathrm{pNa}^{+}$and a $\left.\Delta \psi\right)$. Buffer 1: 20 $\mathrm{mM}$ HEPES/NaOH pH 6.6, $50 \mathrm{mM} \mathrm{Na}_{2} \mathrm{SO}_{4}, 1 \mathrm{mM} \mathrm{AgNO}$ (orange and black curve for CusA wild type proteoliposomes and control liposomes, respectively); Buffer 2: $20 \mathrm{mM}$ HEPES pH 7.6/KOH, $50 \mathrm{mM} \mathrm{K}_{2} \mathrm{SO}_{4}$ (green and magenta curve for CusA wild type proteoliposomes and control liposomes, respectively). CusA wild type proteoliposomes and control liposomes were prepared in buffer: $20 \mathrm{mM}$ HEPES $\mathrm{pH} 7.6 / \mathrm{KOH}, 50 \mathrm{mM} \mathrm{K} \mathrm{SO}_{4}$. Fluorescence of ACMA dye was monitored as a function of time $\left(\lambda_{\mathrm{ex}}=410 \mathrm{~nm} ; \lambda_{\mathrm{em}}=500 \mathrm{~nm}\right.$ ). Fluorescence was normalized to fold change, $\mathrm{F} / \mathrm{F}_{0}$ (division by the initial value). Data points are average of two consecutive measurements $(n=2)$.

Additionally, an experiment was performed where CusA wild type proteoliposomes and control liposomes were subjected to $1 \mathrm{mM} \mathrm{AgNO}_{3}$. Silver nitrate was added after observing a stable fluorescence signal. When an electrochemical gradient (dilution into buffer $1, \mathrm{a} \Delta \mathrm{pNa}^{+}$ (inward directed), a $\Delta \psi$ (negative inside) and a $\Delta \mathrm{pH}$ (acidic outside)) is applied across the membrane of the liposome, and substrate was added, quenching of the ACMA dye was observed for CusA wild type proteoliposomes while no quenching was observed for control liposomes (black and blue curves for CusA wild type proteoliposomes and control liposomes, respectively; Figure 69).

The CusA-mediated transport under conditions where $\Delta \mathrm{pNa}, \Delta \psi$ (negative inside) were imposed led to a quenching over time, as well (red and magenta curves for CusA wild type proteoliposomes and control liposomes, respectively; Figure 69). 


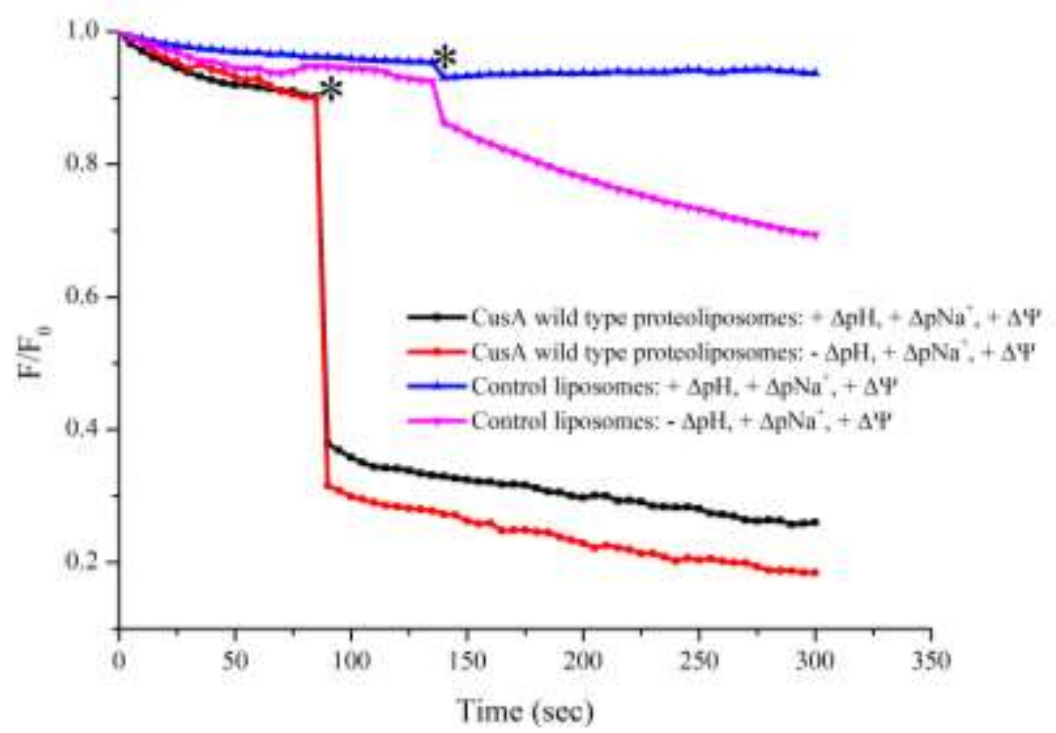

Figure 69. CusA-mediated ACMA quenching. CusA wild type proteoliposomes and control liposomes were subjected to different buffer solutions, imposing (+) or not imposing (-) different driving forces $\left(\Delta \mathrm{pH}\right.$, a $\Delta \mathrm{pNa}^{+}$and a $\left.\Delta \psi\right)$. Buffer 1 : $20 \mathrm{mM}$ HEPES/ $\mathrm{NaOH}$ pH 6.6, $50 \mathrm{mM} \mathrm{Na} \mathrm{SO}_{4}$ (black and blue curve for CusA wild type proteoliposomes and control liposomes, respectively); Buffer 2: $20 \mathrm{mM}$ HEPES pH 7.6/KOH, $50 \mathrm{mM} \mathrm{Na}_{2} \mathrm{SO}_{4}$ (red and magenta curve for CusA wild type proteoliposomes and control liposomes, respectively). CusA wild type proteoliposomes and control liposomes were prepared in buffer: $20 \mathrm{mM}$ HEPES pH 7.6/KOH, $50 \mathrm{mM} \mathrm{K}_{2} \mathrm{SO}_{4}$. (*) indicates addition of $1 \mathrm{mM} \mathrm{AgNO}$. Fluorescence of ACMA dye was monitored as a function of time $\left(\lambda_{\mathrm{ex}}=410 \mathrm{~nm} ; \lambda_{\mathrm{em}}=500 \mathrm{~nm}\right)$. Fluorescence was normalized to fold change, $F / F_{0}$ (division by the initial value).

The observed quenching indicates that when the above-mentioned gradients are applied over the liposome membrane and the substrate (silver ions) is present, proton transport takes place. In opposition to that, when silver ions are not present, no quenching of ACMA is observed (the beginning of the curves, before the * which indicated silver nitrate addition; Figure 69).

\subsubsection{PhenGreen $\mathrm{SK}$ dye as a reporter system of $\mathrm{Ag}^{+}$-transport by CusA reconstituted in liposomes}

The proteoliposomes contain CusA in different orientations and the electrochemical gradient has a different effect on the transport of silver transport and counter-transport of protons. Since the stoichiometry is not known, the differently oriented CusA can produce mixed results.

Interesting observations were made, when CusA wild type proteoliposomes were reconstituted in a different set up, where the $\mathrm{pH}$ gradient was imposed inward. The 
electrochemical gradient was applied onto the liposome's membrane, since the lumen contained potassium ions while the outside buffer contained sodium ions, and valinomycin was used from the beginning of the measurement (a $\Delta \psi$ was imposed (negative inside)).

In this set up, a strong time-dependent quenching of the PGSK was observed when CusA wild type proteoliposomes were diluted into buffer: $20 \mathrm{mM}$ HEPES/NaOH pH 6.6, $50 \mathrm{mM}$ $\mathrm{Na}_{2} \mathrm{SO}_{4}, 1 \mathrm{mM} \mathrm{AgNO}_{3}$ (driving forces: $\Delta \mathrm{pH}, \Delta \mathrm{pNa}^{+}$and $\Delta \psi$ ), suggesting transport of the silver ions into the vesicle (Figure 70, orange curve). Control liposomes without CusA under the same conditions showed a slight quenching in the first $100 \mathrm{sec}$ of the measurements and a gradual dequenching during the next $200 \mathrm{sec}$ (Figure 70, black curve).

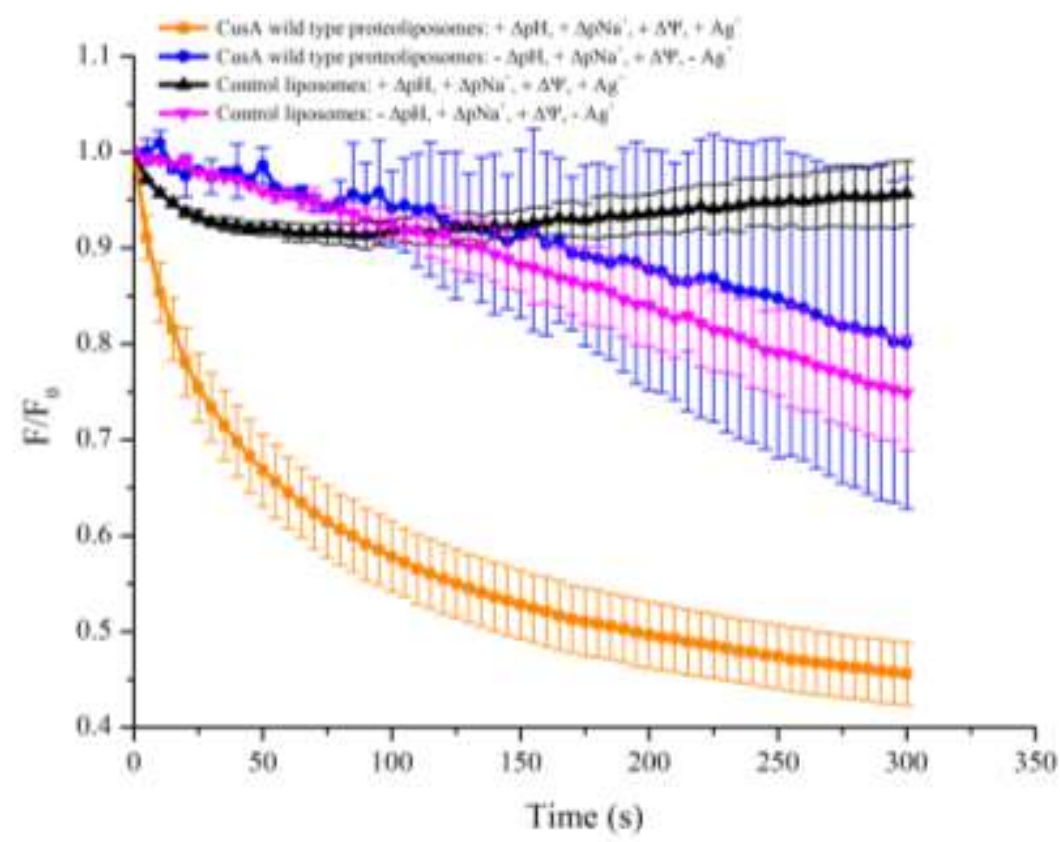

Figure 70. CusA-mediated $\mathrm{Ag}^{+}$-transport in proteoliposomes. CusA wild type proteoliposomes and control liposomes were subjected to different buffer solutions, imposing (+) or not imposing (-) different driving forces $\left(\Delta \mathrm{pH}, \mathrm{a} \Delta \mathrm{pNa}^{+}\right.$and a $\left.\Delta \psi\right)$. Buffer 1: $20 \mathrm{mM}$ HEPES/NaOH pH 6.6, $50 \mathrm{mM} \mathrm{Na} 2 \mathrm{SO}_{4}, 1 \mathrm{mM} \mathrm{AgNO}$ (orange and black curves for CusA wild type proteoliposomes and control liposomes, respectively); Buffer 2: $20 \mathrm{mM}$ HEPES pH 7.6/NaOH, $50 \mathrm{mM} \mathrm{Na}_{2} \mathrm{SO}_{4}$ (blue and magenta curve for CusA wild type proteoliposomes and control liposomes, respectively). CusA wild type proteoliposomes and control liposomes were prepared in buffer: $20 \mathrm{mM}$ HEPES pH 7.6/KOH, $50 \mathrm{mM} \mathrm{K} 2 \mathrm{SO}_{4}$. Fluorescence of PGSK dye was monitored as a function of time $\left(\lambda_{\mathrm{ex}}=480 \mathrm{~nm} ; \lambda_{\mathrm{em}}=530 \mathrm{~nm}\right)$. Fluorescence was normalized to fold change, $F / \mathrm{F}_{0}$ (division by the initial value). Data points are average of: three consecutive measurements $(n=3)$ for CusA wild type proteoliposomes and control liposomes in buffer 1 , two consecutive measurements $(n=2)$ for CusA wild type proteoliposomes and control liposomes in buffer 2 .

CusA-containing proteoliposomes did not show transport of $\mathrm{Ag}^{+}$, as expected, in absence of $\mathrm{Ag}^{+}$and when only $\Delta \mathrm{pNa}^{+}$and $\Delta \psi$ were applied (Figure 70 , blue curve). In the control liposomes there was a similar linear decrease of the fluorescence in the absence of $\mathrm{Ag}^{+}$in the assay (Figure 70, magenta curve). 
Since silver ion transport was detected (by quenching of PGSK, Figure 70, orange curve), while direction of the $\mathrm{pH}$ gradient and the electric gradient is directed towards inside of the proteoliposomes (the $\Delta \psi$ - negative inside), this would suggest $\mathrm{Ag}^{+} / \mathrm{H}^{+}$symport. As shown in the experiment earlier in the chapter (Figure 67), $\Delta \psi$ in absence of $\Delta \mathrm{pH}$ strongly facilitates $\mathrm{H}^{+}$influx as well.

For the determination of basal activity of CusA that is orientated inside-out, PGSK dye was used. The functional assay was designed where the buffer inside the liposomes was acidic (20 $\mathrm{mM}$ HEPES/NaOH $\mathrm{pH}$ 6.6) and the source of protons, while the buffer outside the proteoliposomes was basic (20 mM HEPES/NaOH pH 7.4) and it contained silver ions in a form of $1 \mathrm{mM}$ silver nitrate. For the control, empty vesicles were used, where CusA is not reconstituted. This experiment was done by Miles Willoughby as a part of his Bachelor thesis (under my supervision). This idea for the assay comes from the study done by Long et al in 2010 (Long et al., 2010). In the mentioned study, CusA wild type was used in order to investigate the activity of the protein and was shown that CusA is able to transport metal ions directly from the cytoplasm. Stop-flow transport assays of reconstituted CusA showed the quenching of PGSK, which is incorporated in the proteoliposome. When silver ions are in the outside buffer and acidic buffer is inside the vesicle, the inside-out oriented CusA is energized thus the cytoplasmic transport is activated. silver ions are transported inside the vesicle and quench the dye (Long et al., 2010). Proton influx that was investigated in this study (5.2.2.5), addresses the right-side out oriented CusA, whereas with PGSK, the insideout ones are addressed. For the investigation of the inside-out CusA, $\Delta \mathrm{pH}$ is inverted in compassion to the experiments investigating the right-side out CusA. 


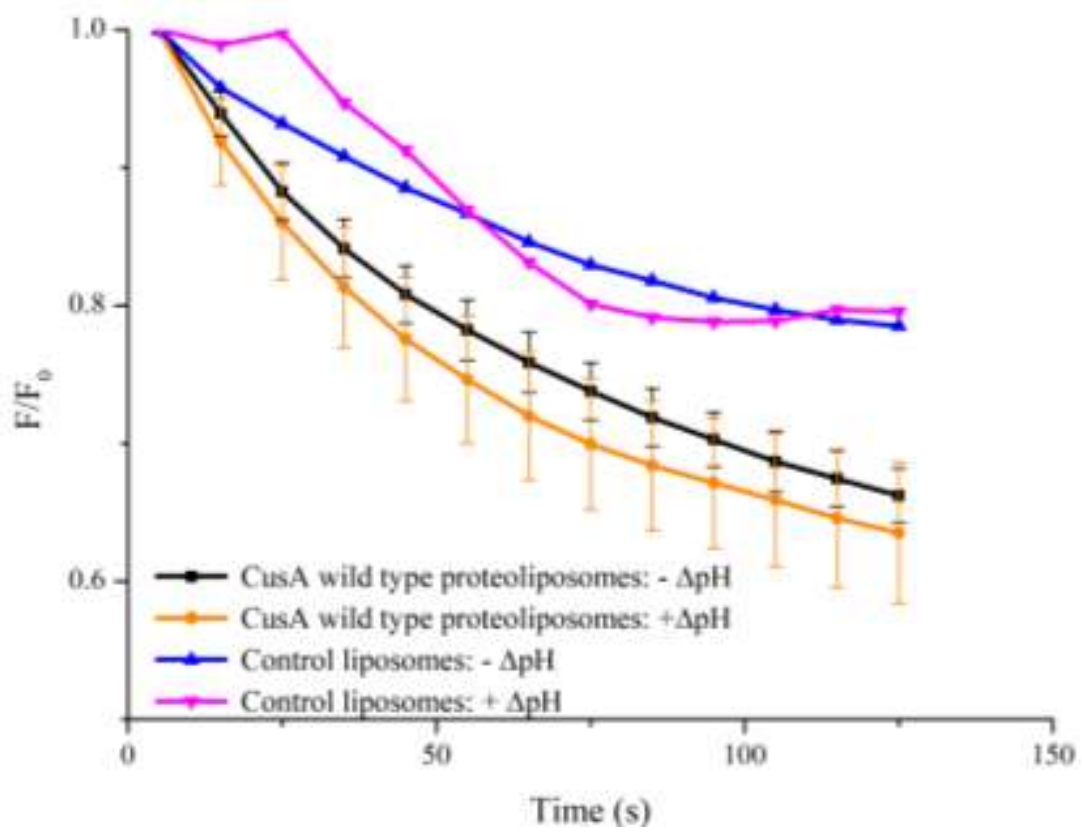

Figure 71. $\mathrm{Ag}^{+}$-transport into CusA-containing proteoliposomes. CusA wild type proteoliposomes and control liposomes were subjected to different buffer solutions, imposing (+) or not imposing (-) an outwardly-directed $\Delta \mathrm{pH}$. Buffer 1: $20 \mathrm{mM}$ HEPES/NaOH pH 6.6 (black and blue curve for CusA wild type proteoliposomes and control liposomes, respectively);

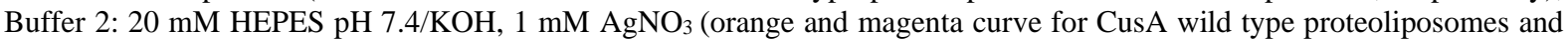
control liposomes, respectively). CusA wild type proteoliposomes and control liposomes were prepared in buffer: $20 \mathrm{mM}$ HEPES pH 6.6/ $\mathrm{NaOH}$. Fluorescence of PGSK dye was monitored as a function of time $\left(\lambda_{\mathrm{ex}}=480 \mathrm{~nm} ; \lambda_{\mathrm{em}}=530 \mathrm{~nm}\right)$. Valinomycin was not used in this experiment. Fluorescence was normalized to fold change, $\mathrm{F} / \mathrm{F}_{0}$ (division by the initial value). Data points are average of: for CusA wild type proteoliposomes five consecutive measurements in reconstitution buffer $(n=5)$ and nine consecutive measurements in assay buffer $(n=9)$, while control liposomes are shown as single measurements.

In the results shown in Figure 71, CusA wild type proteoliposomes (inside buffer at $\mathrm{pH}$ 6.6) show quenching of the PGSK dye when diluted in the basic buffer ( $\mathrm{pH} 7.4$ ) that contains silver ions, in comparison with the control liposomes (Figure 71, orange and magenta curve, respectively). Quenching is also observed, but less steep as in the presence of a $\Delta \mathrm{pH}$, when CusA wild type proteoliposomes are being diluted in the reconstitution buffer $(\mathrm{pH} 6.6$, the same as inside the proteoliposome), which indicates silver ion transport in the absence of a pH gradient (Figure 71, black line). For the control liposomes without CusA, a noticeable but clearly less prominent quenching is observed in absence of a $\Delta \mathrm{pH}$ (Figure 71, blue curve). Valinomycin was not used in this experiment. 


\subsubsection{Discussion}

\subsubsection{Reconstitution efficiency of CusA wild type}

CusA wild type was reconstituted in artificial membranes in two different manners: first with freeze and thaw cycles alone, and second - freeze and thaw cycles together with the PGSK dye, after which proteoliposomes were passed through a gel filtration column, for removal of any residual dye on the surface of the (proteo)liposomes. Since additional steps were taken during reconstitution that involves the PGSK dye (additional freeze-thaw cycles, gel filtration), the reconstitution efficiency was compared between proteoliposomes prepared with PGSK and proteoliposomes that would incorporate the ACMA dye.

The difference in the proteoliposome preparation comes from the fact that unprotonated ACMA freely diffuses across the membrane and upon protonation remains trapped inside the proteoliposomes. The increase of total ACMA (protonated and unprotonated) inside the proteoliposome causes quenching of the fluorescent signal. The dye is added shortly before the measurement. PGSK, on the other hand, is a soluble dye and does not traverse the membrane. It has to be incorporated inside the (proteo)liposomes by freeze and thaw cycles during the reconstitution process.

The solubilization of both batches of prepared liposomes was performed in ordered to determine how much protein was successfully incorporated into the liposome. For the ACMA assay, calculations showed $36 \%$ CusA which could be successfully solubilized from the proteoliposomes, while for PGSK assay this number was approx. $57 \%$. The reconstitution efficiencies showed however, much lower values (13\% for ACMA assay preparation and $14 \%$ for PGSK assay preparation). Also, for the PGSK assay additional steps of freeze and thaw cycles were performed, which may influence that the protein is incorporated more effectively. 


\subsubsection{CusA wild type reconstituted into liposomes shows activity}

Proteins reconstituted in liposomes can take one of two orientations: the right-side out and the inside-out orientation. The right-side out, cell-like, orientation means that the periplasmic part of the protein is oriented outside the liposomes, while the inside-out orientation means that the periplasmatic part is oriented inside the liposome. Which orientation is preferred, highly depends on the reconstitution method and it could vary batch-to-batch.

The successful production of CusA wild type, from the amplification of the cusA gene from the genome of E. coli until the purification (Figure 59), enabled the investigation of the activity of this protein in an in vitro system.

The data presented here are indicating that CusA wild type can transport silver ions over the membrane. In order to show the periplasmic transport, a proton sensitive dye was incorporated inside the liposome, in which the protein is reconstituted. When silver ions are present outside of the proteoliposome, the activity of right-side out oriented CusA is triggered to transport protons to the inside of the proteoliposome. These indirect observations of the silver ion transport were compared with liposomes that are empty (no protein is reconstituted) as well as with the behaviour of the CusA D405N variant, that carries a mutation in the proton relay network and should be inactive. In this study the $\mathrm{D} 405 \mathrm{~N}$ variant did not show complete loss of activity. It might be that the mutation $\mathrm{D} 405 \mathrm{~N}$ is not sufficient for the complete inactivation of proton transfer which extends the finding of Long et al from 2010, where they showed that the D405A variant is unable to transport silver ions due to its inactivity (Long et al., 2010). Franke et al. showed that the D405N variant is inactive (it shows same MIC values as $\triangle c u s A)(F r a n k e$ et al., 2003). The results from Franke et al., together with the data from Long et al., seem to be in contrast with the data presented here.

The data presented here showed various behaviour patterns of CusA proteoliposomes. In the first experiments performed, a $\mathrm{pH}$ gradient was imposed onto the system. In this manner, proteoliposomes were reconstituted with the buffer inside the vesicle with $\mathrm{pH} 7.6$ or $\mathrm{pH}$ 6.6, and the same proteoliposomes were then diluted into the buffers with either $\mathrm{pH} 6.6$ and/or $\mathrm{pH}$ 7.6 respectively (Figure 63). This setup gave insight into the influence of the $\mathrm{pH}$ gradient as a stimulus for the CusA machinery. The transport of protons would create an electrical gradient that would presumably counter the driving force of the inward-facing proton transport. Next 
to that, there would be a small electrical gradient due to a difference in potassium ion concentration. This difference is caused by $\mathrm{KOH}$ titration to set the $\mathrm{pH}$ of the buffers. Using Henderson-Hasselbalch equation, a pH of 6.6 is achieved by adding $2.5 \mathrm{mM} \mathrm{KOH}$, while a $\mathrm{pH}$ of 7.6 is achieved with adding $25.2 \mathrm{mM}$ of $\mathrm{KOH}$. This difference is in the millimolar range $\left(\Delta\left[\mathrm{K}^{+}\right]\right.$is $\left.22.7 \mathrm{mM}\right)$ when the $\mathrm{pH}$ gradient over the membrane is 1 . Considering also the $50 \mathrm{mM} \mathrm{K}_{2} \mathrm{SO}_{4}$ on both sides of the proteoliposome membrane, the electrical gradient is approximately $-5.1 \mathrm{mV}(-59 * \log 125.2 / 102.5)$. This electrical gradient is very small and it can be neglected.

Due to this small difference, it can be assumed that it does not have a significant impact. The most notable quenching of the $\mathrm{pH}$ sensitive dye incorporated inside the proteoliposome (ACMA) was detected with the CusA wild type proteoliposomes that are reconstituted with the buffer $\mathrm{pH} 7.6$ and diluted in the buffer $\mathrm{pH} 6.6$ containing silver ions. If it is assumed that CusA is active, and has a periplasmic transport activity, protons are then transported inside the vesicle, while $\mathrm{Ag}^{+}$is present outside the proteoliposomes. Silver ions will enter the periplasmic pathway and most likely bind to the methionine cluster (M573/M623/M672). This likely primes CusA in the $\mathrm{H}^{+}$-transport competent stage in analogy with the drug/ $\mathrm{H}^{+}$ antiport cycle described for the E. coli efflux pump AcrB (Figure 72).

Once the proton binds to the proton relay site of CusA, conformational changes will trigger the release of $\mathrm{Ag}^{+}$from the binding site, which in vivo would result in $\mathrm{Ag}^{+}$to be transported into the $\mathrm{CusB} / \mathrm{CusC}$ tunnel towards the outside. In the proteoliposomes with only CusA present, it will initiate the release of $\mathrm{Ag}^{+}$on the same site from which it is sequestered, resulting in a futile cycle. 


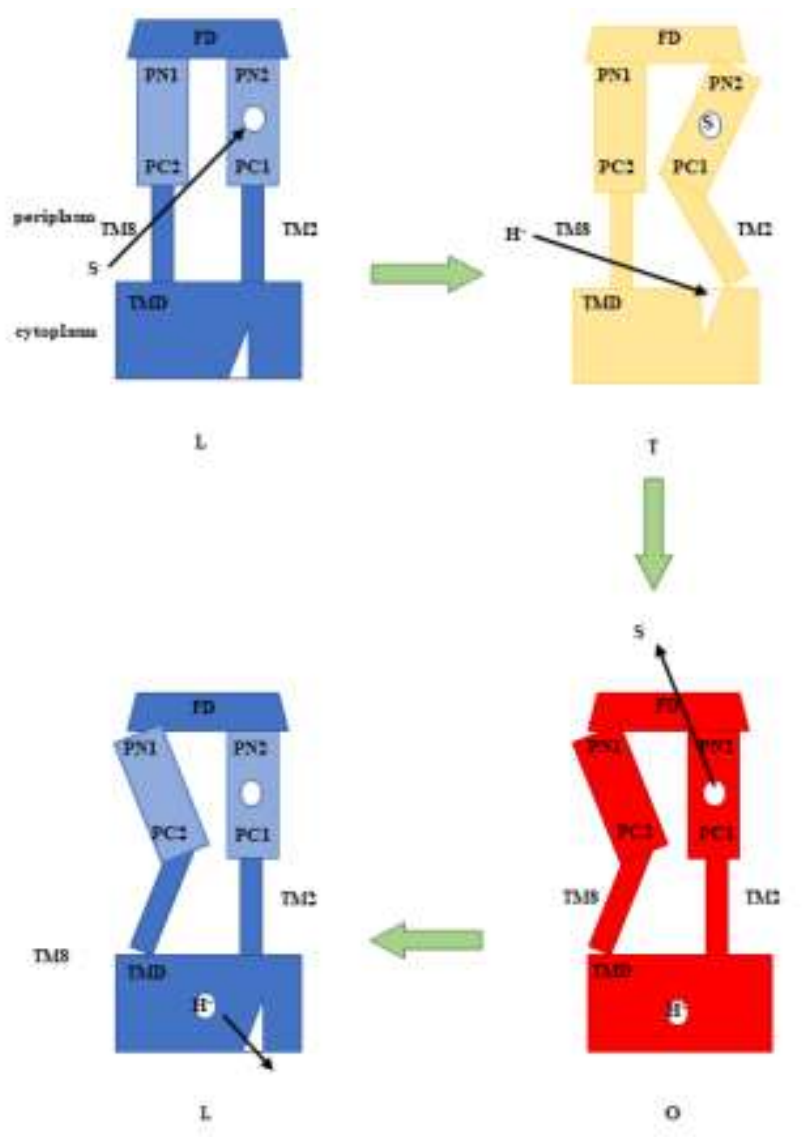

Figure 72. Mechanism of substrate-proton antiport in AcrB. The scheme indicates the conformational cycle Loose-TightOpen-Loose (L-T-O-L) of a single protomer within the AcrB trimer. L-blue, T-yellow, O-red. Transmembrane domain (TMD). FD-funnel domain. S-substrate. The subdomains PC1/PN2 and PN1/PC2 move during the substrate-drug transport. TM2 and TM8 are mediating the conformational changes in periplasmatic and transmembrane domain. Arrows indicating the substrate transport and protonation and deprotonation. Adapted from (Reinke Tobias Müller \& Pos, 2015).

The CusA D405N variant was used as a control for the activity check-up of CusA wild type. When CusA D405N proteoliposomes were diluted in the acidic buffer $(\mathrm{pH}$ inside the proteoliposomes 7.6, $\mathrm{pH}$ outside the proteoliposomes 6.6) quenching of ACMA dye was noticed, indicating the transport of protons towards the interior. The quenching is not as notable as it is for CusA wild type. These observations can indicate that CusA D405N is not as inactive as it is predicted, and that maybe additional mutations in the proton relay network are needed in order to shut down the activity of the pump. The second assumption can be that the mechanical incorporation of the protein made the liposomes leaky, since the empty liposomes do not show as notable quenching. However, when CusA wild type was diluted in a buffer that does not impose any gradient, no quenching of ACMA was detected, indicating that there is no $\mathrm{H}^{+}$influx, as expected (Figure 66). These results lead to suggestions for future in vitro, proteoliposomes experiments (using fluorescence probe as a reporter system in a 
stop-flow setup) to be done on CusA D405N variant and other inactive variants as used by Long et al (Long et al., 2010). Further improvement of the reconstitution of CusA D405N should be done as well as experiments in different buffer conditions in order to show the potential inactivity and prove the CusA wild type activity. These experiments should verify additional conditions such as proteoliposomes with driving forces in the presence/absence of silver ions, proteoliposomes without driving force in the presence/absence of silver ions and the same conditions with empty liposomes and inactive variants (from proton relay network when observing proton transport and from methionine pathway when observing silver transport).

Control liposomes (empty liposomes, no protein is being reconstituted) are checked for the behaviour as well. Two setups are being checked, when the $\mathrm{pH}$ inside the liposome is $\mathrm{pH} 6.6$ and outside $\mathrm{pH}$ 7.6, as well as the reversed setup which shows the periplasmatic transport pH 7.6 inside and pH 6.6 outside the liposome. The control liposomes and CusA wild type proteoliposomes in the condition $\mathrm{pH} 6.6$ inside and $\mathrm{pH} 7.6$ outside the vesicle do not show a decrease of the fluorescence signal (blue and pink curve, respectively, Figure 64) in comparison to the signal decrease of the CusA wild type proteoliposomes with $\mathrm{pH} 7.6$ inside and pH 6.6 outside (orange curve, Figure 64). In the latter case, control liposomes do not show quenching of the fluorescence as well (black curve, Figure 64). These results could indicate that CusA wild type is active when reconstituted in the lipid bilayer and that in the setup where silver ions are available outside proteoliposomes, CusA right-side out orientated would be energized for the transport of the substrate in the periplasmic transport fashion (Figure 65). To validate this hypothesis, and check if the influx of protons is indeed dependent on the presence of silver, the assay should be repeated without silver ions added outside.

Further experiments, shown in Figure 67, used CusA wild type proteoliposomes that were subjected to an additional driving force applied to the system. Valinomycin was used to create an electrical gradient (negative inside), because of the different concentrations of $\mathrm{K}^{+}$ across the membrane. Valinomycin is a specific $\mathrm{K}^{+}$ionophore and allows efflux of potassium ions along its gradient. The chemical gradient of potassium ions is then converted into an electrical gradient in the opposite direction. Three buffers were used to show the influence of the components of the electrochemical gradient on the system. The proteoliposomes were prepared in $20 \mathrm{mM}$ HEPES $\mathrm{pH} 7.6 / \mathrm{KOH}, 50 \mathrm{mM} \mathrm{K} \mathrm{SO}_{4}$. When diluted into $20 \mathrm{mM}$ 
HEPES/NaOH pH 7.6, $50 \mathrm{mM} \mathrm{Na} \mathrm{SO}_{4}, 1 \mathrm{mM} \mathrm{AgNO}$ (buffer 3), an electrical gradient (inside negative) was imposed. Dilution into $20 \mathrm{mM}$ HEPES pH 7.6/KOH, $50 \mathrm{mM} \mathrm{K} \mathrm{SO}_{4}$ (buffer 2) served as a control where no gradient is imposed. When buffer 1 was used, $20 \mathrm{mM}$ HEPES/NaOH pH 6.6, $50 \mathrm{mM} \mathrm{Na} \mathrm{SO}_{4}, 1 \mathrm{mM} \mathrm{AgNO}$, an inwardly directed pH gradient and an electrical gradient (negative inside) were imposed. As shown on Figure 67, when inside and outside buffer are the same and hence no gradient is applied over the membrane, there is no quenching of ACMA. This represents the state where no protons are transported into the liposomes. This leads to the conclusion that the protein is inactive in the absence of its driving force. In difference to that, when there is a chemical gradient and electrical gradient present (induced by usage of buffer 3) quenching of the dye indicates the transport of protons into the lumen. Clearly, the force of the electrical gradient and the substrate present outside the proteoliposome are enough to activate the protein to transport the ions. With the addition of a pH gradient (buffer 1) quenching is present as well, implying that either an electrochemical gradient or both a $\mathrm{pH}$ and an electrochemical gradient can induce proton transport.

It is important to test how control liposomes, liposomes with no protein incorporated into the membrane, are behaving in the condition that can be the potential condition for the assay. The control liposomes (Figure 68) were diluted into the buffer 2, $20 \mathrm{mM}$ HEPES pH 7.6/KOH, $50 \mathrm{mM} \mathrm{K} \mathrm{SO}_{4}$, which is the reconstitution buffer, and no gradient is applied to the membrane. In this case no quenching of ACMA is observed. The control liposomes were diluted into the buffer 1 (20 mM HEPES/ $\mathrm{NaOH} \mathrm{pH}$ 6.6, $50 \mathrm{mM} \mathrm{Na}_{2} \mathrm{SO}_{4}, 1 \mathrm{mM} \mathrm{AgNO}$ ) resulting in a $\mathrm{pH}$ gradient, a sodium ion gradient and, in the presence of valinomycin, an electrical gradient. In this case slight quenching is noticed, that is presumable due the leakage of $\mathrm{H}^{+}$into the liposomes. In this assay setup, valinomycin is present from the beginning, inserted into the liposome membrane.

Further experiments included addition of the substrate, silver ions, after monitoring the fluorescent signal of CusA wild type proteoliposomes and control (empty) liposomes in the buffer that applies gradients over the liposome membrane but without substrate. Upon addition of silver nitrate, observed quenching of ACMA indicates that when the gradients are imposed (a $\Delta \mathrm{pNa}^{+}$and a $\Delta \Psi$ with/without $\Delta \mathrm{pH}$ ), proton transport takes place (Figure 69). In opposition to that, when silver ions are not present, no quenching of ACMA is observed (the beginning of the curves, before the * which indicated silver nitrate addition; Figure 69). Also, 
control liposomes do not show substantial quenching. This indicates that CusA-mediated proton translocation requires the presence of a substrate (silver ions) and a driving force in form of a gradient (a $\Delta \mathrm{pNa}^{+}$and a $\Delta \Psi$ with/without $\Delta \mathrm{pH}$ ). Interestingly, the $\mathrm{pH}$ gradient is not crucial and even seems to hinder the proton transport (black curve with $\mathrm{pH}$ gradient and red curve without $\mathrm{pH}$ gradient, Figure 69). The force of the electrical gradient and the substrate present outside the proteoliposome are enough to activate the protein to transport the ions. Either an electrochemical gradient or both $\mathrm{pH}$ and electrochemical gradient can induce proton transport. The possible leakage of CusA proteoliposomes due to incorporation of the protein into the membrane in this case can be excluded since the difference in fluorescence between the proteoliposomes and the control (empty) liposomes is minor. Only the addition of substrate triggers a prominent quenching but only in the case of proteoliposomes. All these observations indicate that proton transport is mediated by substrate activated CusA.

\subsubsection{Transport of silver ions by CusA wild type proteoliposomes}

It has been shown that CusA wild type transporter can sequester and transport silver ions directly from the cytoplasmic site of CusA (Long et al., 2010). Similar results are indicated in the work presented here. Figure 71 shows the transport of silver ions which implies that CusA wild type which are oriented in the inside-out orientation (cytoplasmic part of CusA is at the outside of the proteoliposomes) are likely to be energized. The assay buffer had a $\mathrm{pH} 7.4$ while the buffer inside the proteoliposome was $\mathrm{pH} 6.6$ and silver was present outside the proteoliposomes. This setup would trigger the transport of silver ions across the membrane into the liposomes where the ions would quench the metal sensitive dye PGSK. Based on the direction of the $\mathrm{pH}$ gradient applied, protons should be transported out of the proteoliposome. When proteoliposomes are diluted into the buffer with $\mathrm{pH} \mathrm{7.4,} \mathrm{quenching} \mathrm{of} \mathrm{the} \mathrm{metal}$ sensitive dye is observed (orange curve, Figure 71). Control liposomes (empty liposomes) do not show quenching as prominent as proteoliposomes, which indicates that silver transport in proteoliposomes occurred. Together with the ACMA proteoliposome assay (5.2.2.5), the PGSK proteoliposome assay (5.2.2.6) gives insights into the transport of protons and silver ions in the presence of CusA. The quenching observed with CusA wild type proteoliposomes in the buffer that imposes $\Delta \mathrm{pH}$ (Figure 71), suggests that CusA takes up silver ions from the 
outer medium, meaning, CusA possibly transports silver ions from the cytoplasm to the periplasm as well.

The control (empty) liposomes (Figure 71) as well CusA proteoliposomes show to some extent a drop in the fluorescence signal in the absence of driving force, which could indicate possible leakage of silver ions over the membrane. Additionally, the presence of the CusA trimer in the membrane could somehow destabilizes the bilayer so that more ions can cross over. When the driving forces are applied, that would mean additional quenching of PGSK, since silver ions would be transported inside the (proteo)liposomes and quench the incorporated dye. The RND type proteins require a proton gradient to transport substrates, in this case a $\Delta \mathrm{pH}$ of 0.8 , which is outward-oriented (inside acidic). It seems that CusA activity adds to the observed potential leakage, increasing the quenching of the dye inside the proteoliposome.

Additionally, to this experiment (results from Figure 70), CusA wild type was reconstituted in liposomes containing PGSK, with a buffer inside the proteoliposome with $\mathrm{pH}$ 7.6, while the proteoliposomes were diluted in the acidic buffer ( $\mathrm{pH}$ 6.6). Beside the $\mathrm{pH}$ gradient, an electrical gradient was imposed too, with the usage of valinomycin, since potassium ions were present inside the liposomes, and sodium ions in the outside buffer. As shown in Figure 70, CusA wild type proteoliposomes diluted into the buffer with $\mathrm{pH} 6.6$ containing silver, show a significant decrease in the fluorescent signal in comparison to the control liposomes (orange and black curve, respectively). When diluted into the buffer with the same $\mathrm{pH}$ and no substrate present, no significant decrease in fluorescence is observed. In the setup presented in Figure 70, acidic buffer and the substrate are outside the proteoliposomes, and alkaline buffer inside, which is more likely to induce periplasmic transport (i.e., influx of $\mathrm{H}^{+}$into the proteoliposomes, binding and release of $\mathrm{Ag}^{+}$at the periplasmic part of CusA at the outside of the proteoliposomes). As shown on Figure 70 (orange curve) silver ions are transported inside the proteoliposome, implying that in this case the right-side out oriented CusA was energized.

This condition included an inward-directed $\Delta \mathrm{pH}$ and a $\Delta \Psi$ (negative inside) imposed across the membrane. Under similar conditions that included inward-directed $\Delta \mathrm{pH}$ and a $\Delta \Psi$ (negative inside), the acidification of the CusA proteoliposomes was tested and a strong quenching of the $\mathrm{H}^{+}$-sensitive dye ACMA was observed. This might lead to the conclusion that the imposed $\Delta \Psi$ (negative inside) is converted into an inverse $\Delta \mathrm{pH}$ (acidic inside) by diffusion of protons across the membrane, driving the antiport $\left(\mathrm{H}^{+}\right.$out, $\mathrm{Ag}^{+}$in). On the other 
hand, it might imply that both, protons and silver ions are transported inside the proteoliposome in a symport fashion.

Different reporter systems revealed different transport events of CusA in the presence of silver ions outside the proteoliposomes: 1) CusA transports protons into the liposome in the presence of an inward-oriented $\mathrm{pH}$ gradient and/or an inward-oriented electrochemical gradient (Figure 73A); 2) CusA transports silver ions into the liposome in the presence of an outward-oriented $\mathrm{pH}$ gradient (Figure 73B) or 3) in the presence of an inward-oriented $\mathrm{pH}$ gradient together with an inward-oriented electrochemical gradient (Figure 73C). These observations indicate three possible transport pathways for protons and silver ions (Figure 73).

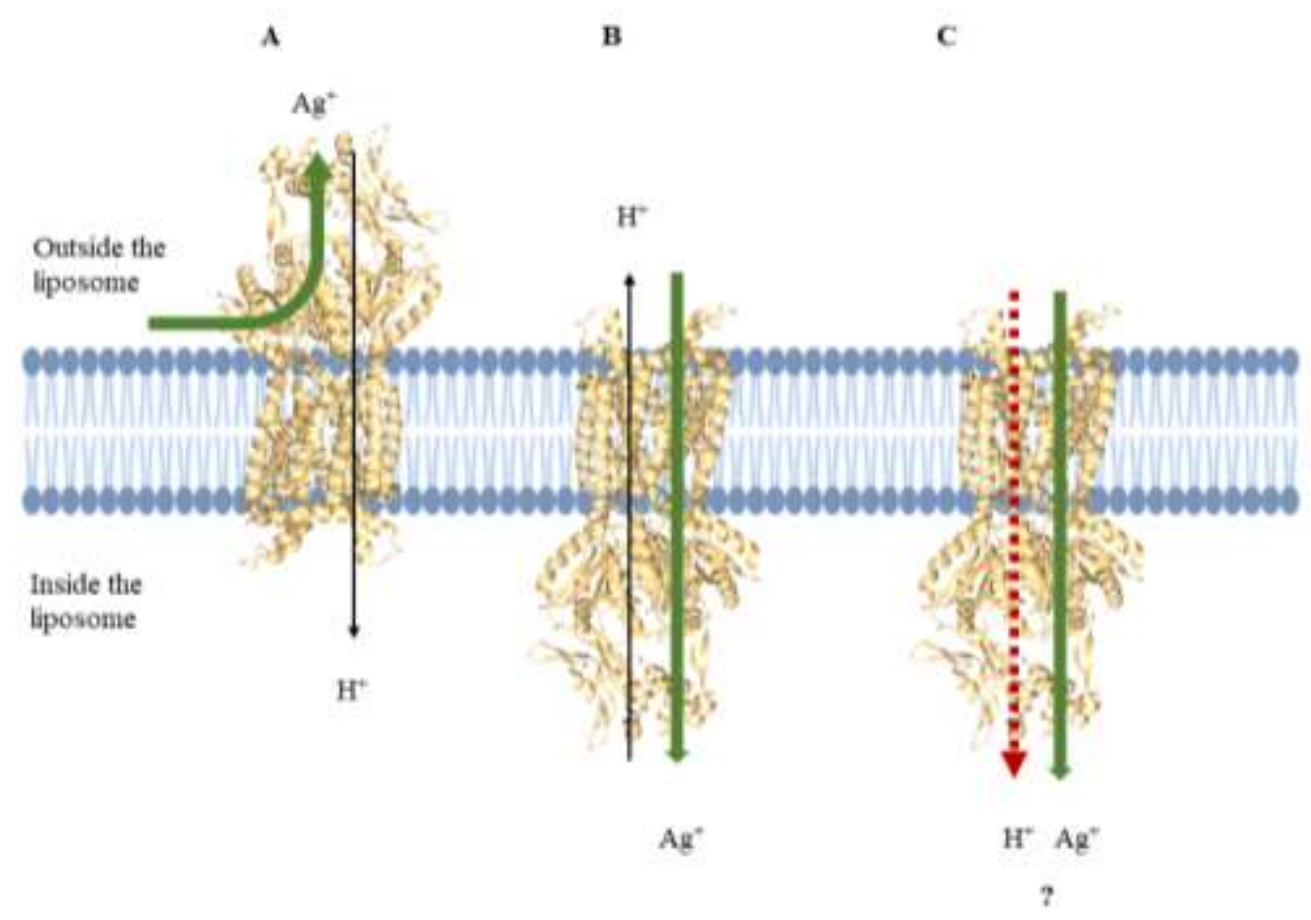

Figure 73. Possible transport pathways of protons and silver ions through CusA efflux pump (PDB: 3K07). CusA monomer was shown. A. Periplasmic transport of silver ions. CusA is right-side out oriented in the liposomes. B. Cytoplasmic-like transport of silver ions. Both $\mathrm{A}$ ) and $\mathrm{B}$ ) are $\mathrm{H}^{+/} \mathrm{Ag}^{+}$antiport. CusA is inside-out oriented in the liposome. C. Proposed cytoplasmic $\mathrm{H}^{+/} \mathrm{Ag}^{+}$symport. CusA is inside-out oriented in the liposome. Figure was prepared with Pymol (https://pymol.org/) and with help of BioRender (https://app.biorender.com/).

When CusA is in right-side out orientation, and $\mathrm{pH}$ gradient is inward-oriented, silver ions available outside the liposome would be transported in a futile cycle, indicating $\mathrm{Ag}^{+} / \mathrm{H}^{+}$ antiport. When CusA is in inside-out orientation in the bilayer, and the $\mathrm{pH}$ gradient is 
outward-oriented (acidic inside the liposome), silver would be transported inside the liposome, indicating cytoplasmic-like transport. When $\mathrm{pH}$ gradient is inward-oriented and silver ions are present outside the liposome, the results shown in Figure 70 are suggesting that $\mathrm{Ag}^{+} / \mathrm{H}^{+}$symport occurs, with transport of both protons and silver ions into the liposome. This experiment has to be tested with an inactive CusA variant.

The initial assumption is that the distribution of protein orientation in liposomes is 50/50 (right-side out to inside-out). Either of the orientations can be energized to be active depending on the orientation of the driving forces. In this work, several different conditions were used and implications obtained regarding which orientation was activated. The results of the transport assays shown in Figure 70 suggest $\mathrm{Ag}^{+} / \mathrm{H}^{+}$symport. If this observation is CusA-mediated, then this would be cautiously analysed in more detail, since RND proteins in general were shown to be antiporters. One example of a RND protein that has been suggested to be a symporter is mammalian NPC1. This protein can be found in the endosome membrane, where it connects the acidic endosome lumen with the cytoplasm (Nikaido, 2018)(Gong et al., 2016). On the other hand, the in vivo orientation of CusA and its in vivo activity as part of the CusCBA complex, clearly suggests that CusA is an antiporter (Long et al., 2010).

Future experiments to validate this hypothesis encompass usage of two reporter systems simultaneously (for example, PGSK and ACMA) to detect proton and silver ion flux at the same time. This set-up allows to monitor specifically the activity of the inside-out oriented CusA. This orientation is energized by an inward-oriented $\mathrm{pH}$ gradient as well as by the presence of the substrate (silver ions) outside the proteoliposomes. Further experiments should focus on the $\mathrm{pH}$ gradient first, by using Valinomycin and potassium-containing buffers. Appropriate controls for these experiments include the usage of an inactive variants of CusA as well as the removal of the driving force, e.g., pH gradient. Additionally, the influence of $\Delta \psi$ as a driving force should be further investigated. 


\section{Chapter VI: Functional analysis of a tripartite Major Facilitator Superfamily (MFS)-type efflux pump EmrAB- $\underline{\text { TolC from } E \text {. coli }}$}

\subsection{Introduction}

Continuous efforts over the past years led to increased knowledge of multicomponent efflux pumps from different families (for example, RND and ABC (super)families), and many details about structure and function are known regarding the pumps AcrAB-TolC, MexABOprM and MacAB-TolC (Du et al., 2014)(Tsutsumi et al., 2019)(Fitzpatrick et al., 2017). However, a lot of things still remain unknown when it comes to this MFS-type efflux pump, EmrAB-TolC. The three-component efflux pump EmrAB-TolC became first the topic of research when the $e m r A B$ operon was first described (O. Lomovskaya \& Lewis, 1992). It was discovered that this operon is a marker for resistance to carbonyl - cyanide mchlorophenylhydrazone (CCCP) and nalidixic acid in E. coli. Previous structural studies showed that the homologues of EmrB from the MFS family, EmrD and MdfA, have 12 transmembrane helices (TMH) (Heng et al., 2015)(Yin, 2006). EmrB from E. coli, on the other hand, has $14 \mathrm{TMH}$ embedded into the membrane (Yousefian et al., 2021).

For the RND-type transporters it is postulated that they sequester the substrates from the periplasmic side of the inner membrane. However, for the EmrAB-TolC it is suggested that the MFS-type transporter, EmrB, captures the substrates from the cytoplasm or the inner leaflet of the inner membrane (Figure 74A). Substrates are then released on the periplasmic side inside the EmrA multimeric adaptor component, connected to the TolC outer membrane channel, leading the toxic compounds out of the cell (Yousefian et al., 2021). 
A)

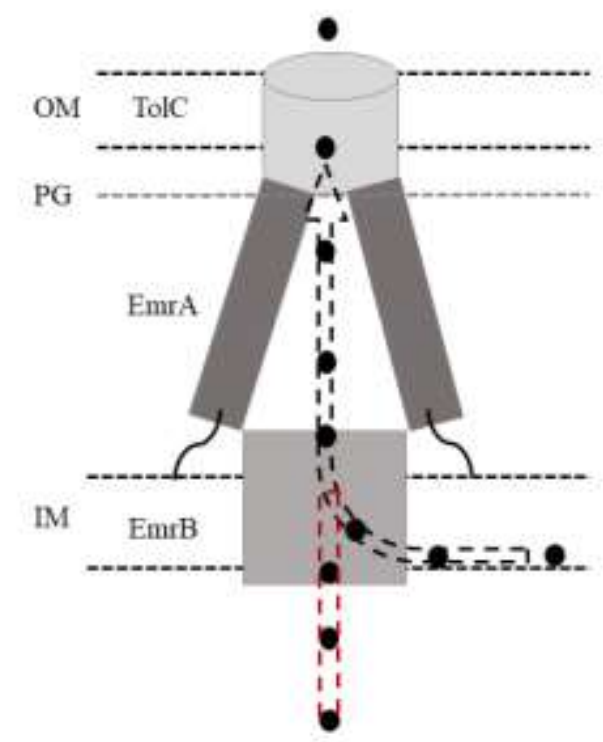

B)

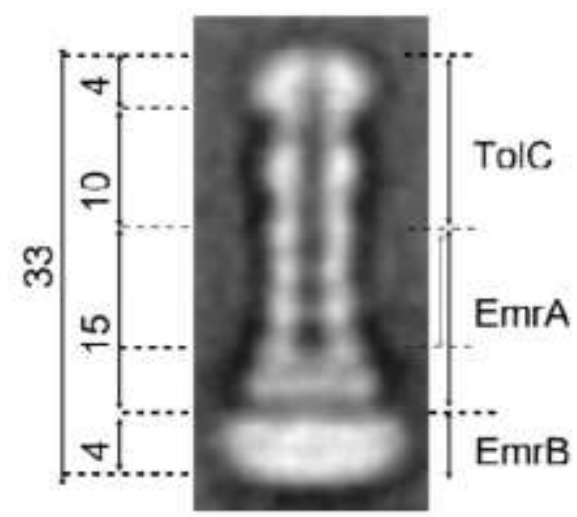

Figure 74. Substrate transport pathway and structural analysis of EmrAB-TolC efflux pump. A. Proposed mechanism of substrate transport through EmrAB-TolC efflux pump. Substrates can be expelled directly from cytoplasm (red dashed path) or from the inner leaflet of the inner membrane of E. coli (black dashed path). After passing through the channel-like EmrA multimeric adaptor component, they are transported out of the cell through the outer membrane channel TolC. B. TEM analysis of the tripartite EmrAB-TolC efflux system. Average image of the EmrAB-TolC complex showing densities corresponding to TolC, EmrA and Apol belts at both extremities (distances between these components are indicated in $\mathrm{nm}$ ). Image taken from (Yousefian et al., 2021), co-author agreement has been obtained.

The purpose of the study shown here is to prove that the fusion proteins used (expressed from pRSFDMG_emrA_tolC and p7XC3RH_emrB) are capable to confer a resistance phenotype which implies that they are able to assemble into a functional tripartite system. This investigation was done to support the structural studies of EmrAB-TolC pump done by Yousefian et al in 2021, which were performed using the same fusion proteins (Figure 74B) (Yousefian et al., 2021).

\subsection{Results}

\subsubsection{Expression of the genes emrA, emrB and tolC}

E. coli strains BW25113(DE3) $\triangle a c r A B$ and C41(DE3) $\triangle a c r A B$, harbouring the constructs pRSFDMG_emrA_tolC and p7XC3RH_emrB (Figure 75) were checked for co-expression. 
Expression levels of EmrA-GFP, EmrB-mRFP1 and TolC-Strep-tag were checked in different $E$. coli strains. The co-expression was tested with different concentrations of the inducer IPTG. The cells of aforementioned strains were disrupted, and the membranes isolated and solubilized (3.2.2.9). The solubilized membranes, harbouring EmrAB-TolC, were subjected to centrifugation, hence the samples shown are taken before and after centrifugation step, respectively.

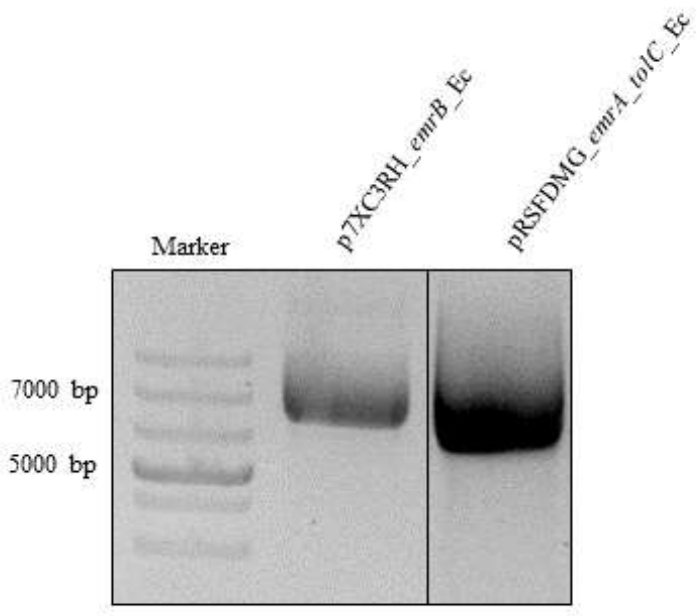

Figure 75. Agarose gel of the vectors harbouring pRSFDMG_emrA_tolC (7280 bp) and p7XC3RH_emrB (7568 bp). The sample were applied on the $1 \%$ agarose gel directly after minipreparation of the respective plasmids. The dye SERVA DNA stain clear G (Serva) was added for later DNA visualization. For visualisation of the DNA, the gel was observed using an UV-transilluminator filter (312 nm) (Intas gel imager).

The plasmid pRSFDMG encodes for EmrA with a C-terminally fused superfolder-GFP variant (sfGFP) and c-Myctag. To monitor the expression of EmrA-GFP, in gel fluorescent for GFP can be used (Figure 76A). Since the vector p7XC3RH harbours the EmrB with a Cterminal mRFP1-His tag fusion protein (red fluorescent protein, mRFP1), the expression of EmrB-mRFP1 can be monitored via in gel fluorescence for mRFP1 (Figure 76B). The plasmid pRSFDMG encodes also a C-terminal StreptagII fused to TolC. For monitoring the expression of TolC, anti-strep tag Western blot was used (Figure 76C). 


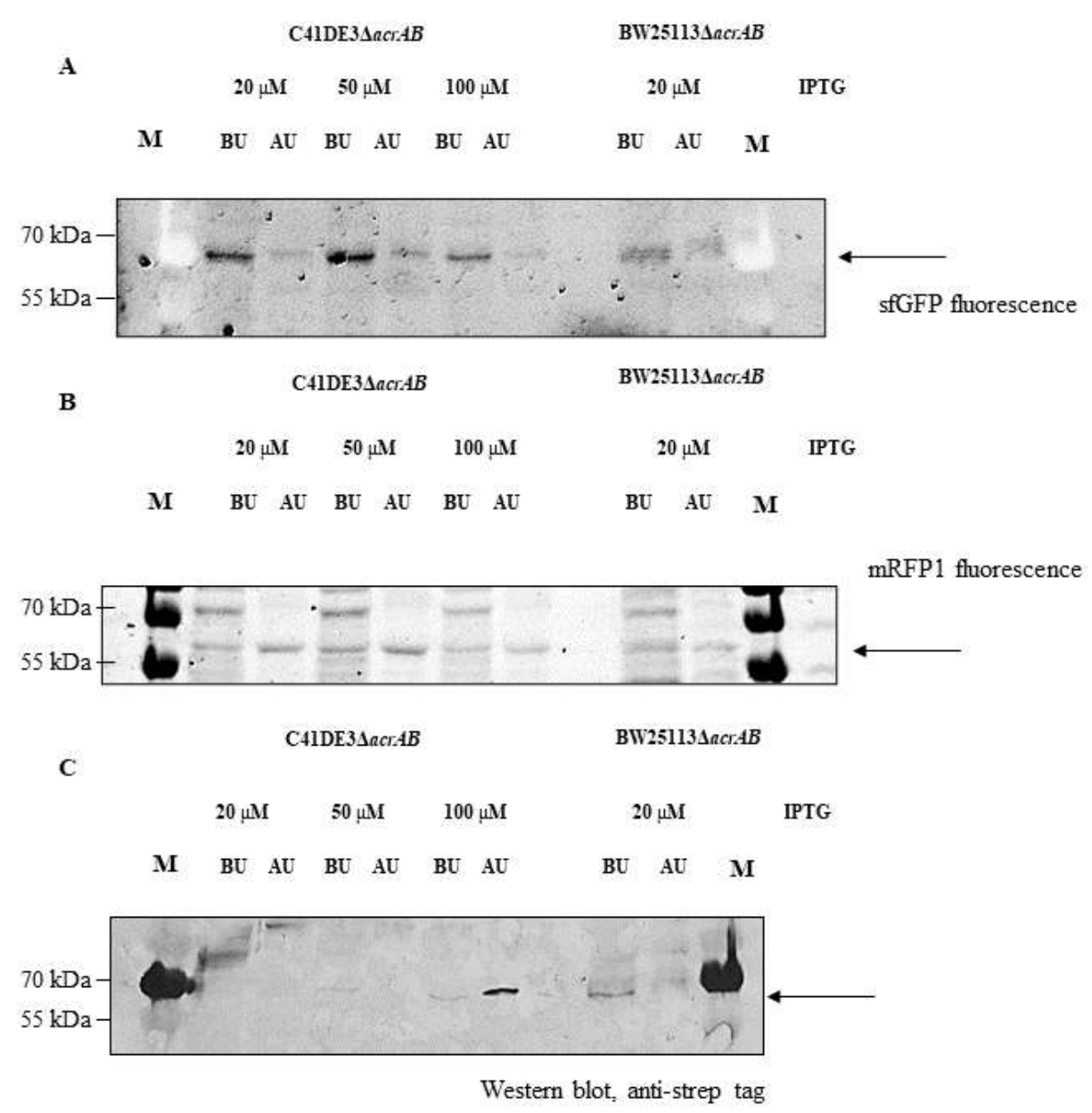

Figure 76. Detection of fusion proteins produced in E. coli. Whole cells producing the fusion proteins were harvested and cell density was normalized to $\mathrm{OD}_{600 \mathrm{~nm}}=10$ and prepared for SDS-PAGE analysis. $20 \mu \mathrm{l}$ of samples induced with $20 \mu \mathrm{M}$ IPTG was subjected to $10 \%$ SDS-PAGE. A. sfGFP fluorescence (for EmrA-sfGFP detection, $\lambda_{\mathrm{ex}} / \lambda_{\mathrm{em}}-460 \mathrm{~nm} / 515 \mathrm{~nm}$ ), B. mRFP1 fluorescence (for EmrB-mRFP1 detection, $\lambda_{\mathrm{ex}} / \lambda_{\mathrm{em}}-630 \mathrm{~nm} / 670 \mathrm{~nm}$ ) and $\mathbf{C}$. Western blot, anti-strep tag antibody (for TolC-Strep-tag detection) (M - marker (PageRuler Prestained Protein Ladder (ThermoScientific)), BU - before centrifugation, $\mathrm{AU}$ - after centrifugation). Arrows indicate the fluorescent (correctly folded) $(\mathrm{A}, \mathrm{B})$ and strep-tagged (C) proteins. Gels were analysed using ImageQuant LAS4000.

\subsubsection{Testing of the $E$. coli strains for the optimization of the substrate concentration of carbonyl cyanide m-chlorophenyl hydrazone (CCCP)}

The functionality of EmrAB-TolC was tested in vivo by a phenotype plate dilution assay (PDA). The initial experiments started with a broad substrate concentration range. Subsequent experiments focused on the representative substrate concentrations. The tests 
were done in order to show that fusion proteins used in this study are forming functional tripartite system. If $E$. coli cells are expressing functional EmrAB-TolC, they would be able to grow in the presence of carbonyl cyanide m-chlorophenyl hydrazone (CCCP).

First, the E. coli bacterial strains, BW25113 wild type and BW25113(DE3) $\triangle a c r A B$ were tested without overexpression of the genes $e m r A, e m r B$ and tolC. The bacterial strains were plated at different cell densities (OD 1-10-5) on solid Müller-Hinton (MH) medium, and each plate contains different concentrations of CCCP. The phenotype of these strains in the presence of different concentrations of this compound is shown in Figure 77.

The concentration below $5 \mu \mathrm{g} / \mathrm{ml}$ of CCCP did not result in the suppression of cell growth for both wild type BW25113 and the variant of the strain lacking the genes acrA and acrB. However, the concentrations around $8 \mu \mathrm{g} / \mathrm{ml}$ showed inhibition of the growth in the cell dilution series between $10^{-2}$ and $10^{-5}$. At $10 \mu \mathrm{g} / \mathrm{ml}$, the $\operatorname{acr} A B$ knockout strain had almost complete growth inhibition, while the wild type still showed growth until the dilution $10^{-3}$.

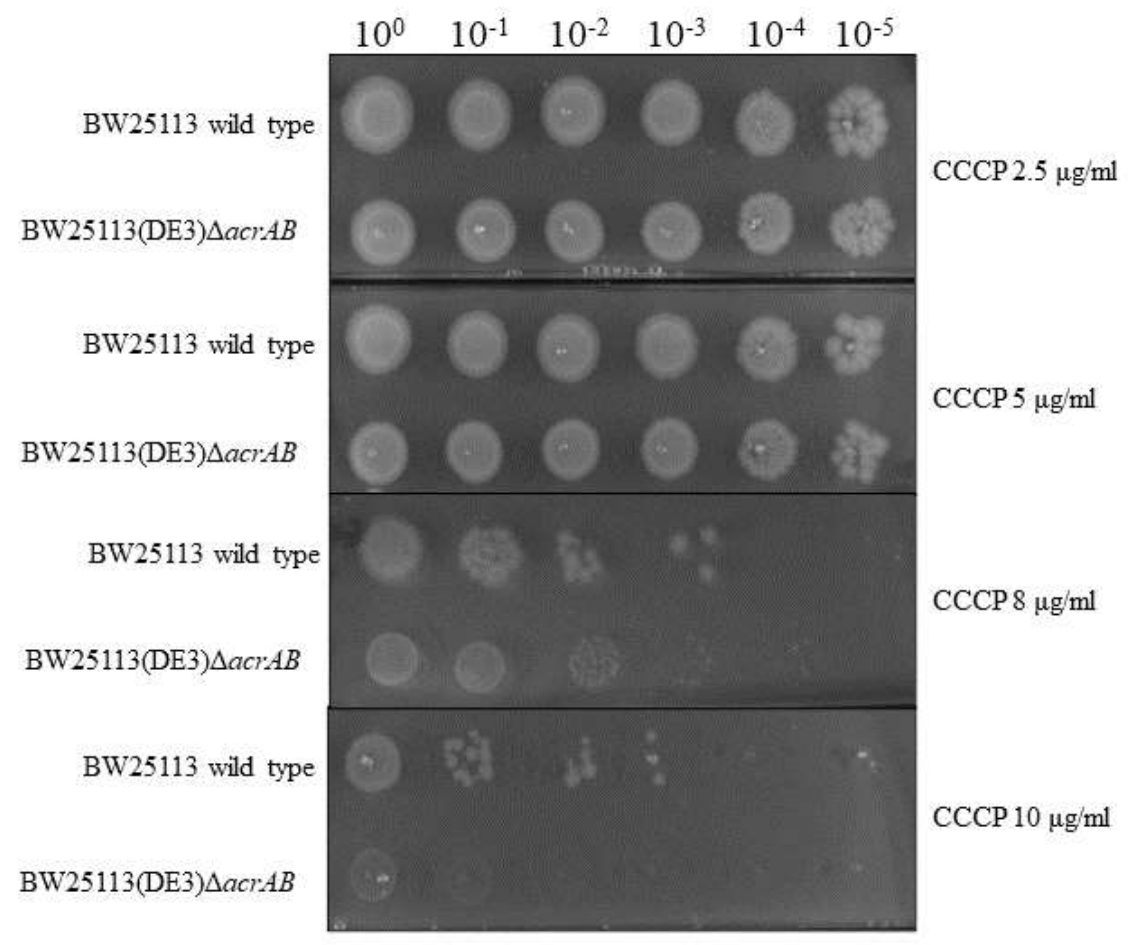

Figure 77. Drug susceptibility profile of $E$. coli BW25113(DE3) $\triangle a c r A B$ strain on solid medium. For the control native, wild type strain BW25113 was used, harbouring no deletion of any gene from the $E$. coli genome and giving the complete picture of the resistance present in the bacteria towards the substrates used. Carbonyl cyanide m-chlorophenyl hydrazone (CCCP) was used at different concentrations for Plate Dilution Assay (PDA). Cells were induced with $20 \mu \mathrm{M}$ IPTG. Culture dilution of cells with $\mathrm{OD}_{600 \mathrm{~nm}}$ of 1 to $10^{0}, 10^{-1}, 10^{-2}, 10^{-3}, 10^{-4}, 10^{-5}$. Results were obtained after $18 \mathrm{~h}$ incubation at $37^{\circ} \mathrm{C}$. Images of the plates were produced with ImagerLAS4000. 


\subsubsection{Co-expression of fusion proteins EmrB-mRFP and EmrA- TolC-GFP forms a functional tripartite system}

The E. coli strain carrying the deletion of the $a c r A B$ locus, BW25113(DE3), was tested on solid medium for the phenotypic characteristics when harbouring the genes emrA, emrB and tolC. The cells of this strain were also transformed with the empty vectors pET24a and pUC19. Vector pET24a has a kanamycin resistance cassette as the vector p7XC3RH that is used for the cloning of the $e m r B$ gene. Vector pUC19 encodes for the ampicillin resistance gene as the vector pRSFDMG that is used for the cloning of the emrA and tolC genes.

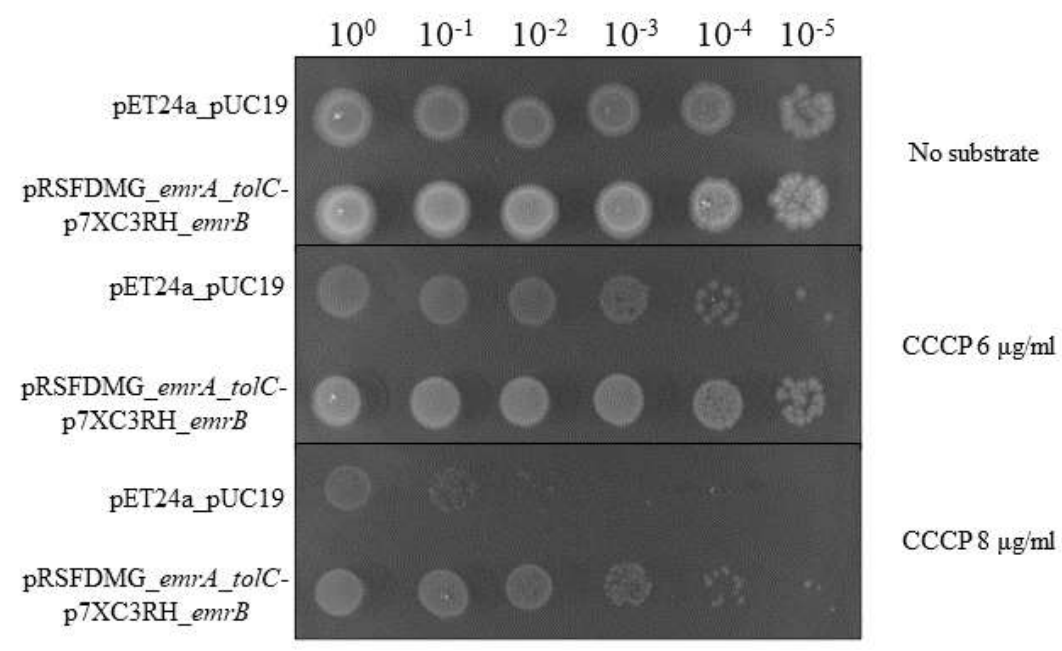

Figure 78. Drug susceptibility test of $E$. coli BW25113(DE3) $\triangle a c r A B$ harbouring pRSFDMG_emrA_tolC and p7XC3RH_emrB. For the control empty vectors that harbour the same resistance cassettes were used, pET24a and pUC19. First observations of the pump activity were made at $6 \mu \mathrm{g} / \mathrm{ml}$ of CCCP. Cells were induced with $20 \mu \mathrm{M}$ IPTG. Culture dilution of cells with $\mathrm{OD}_{600 \mathrm{~nm}}$ of 1 to $10^{0}, 10^{-1}, 10^{-2}, 10^{-3}, 10^{-4}, 10^{-5}$. Results were scored after $18 \mathrm{~h}$ incubation at $37^{\circ} \mathrm{C}$. Images of the plates were produced with ImagerLAS4000.

When no substrate of the EmrAB-TolC pump is present, cells expressing the empty vectors as well as cells expressing the pump comprising red and green fluorescent fusion protein partners, were growing at any dilution applied to the plate. When CCCP was used for the inhibition of the cell growth, cells harbouring the genes encoding for the MDR pump were growing till the dilution of $10^{-4}$ at the concentrations of $8 \mu \mathrm{g} / \mathrm{ml}$, while cells expressing the empty vectors were suppressed to grow at the first dilution step, $10^{-1}$ (Figure 78). Cells harbouring empty vectors show a decrease in the growth capability already at $6 \mu \mathrm{g} / \mathrm{ml}$ of CCCP. 


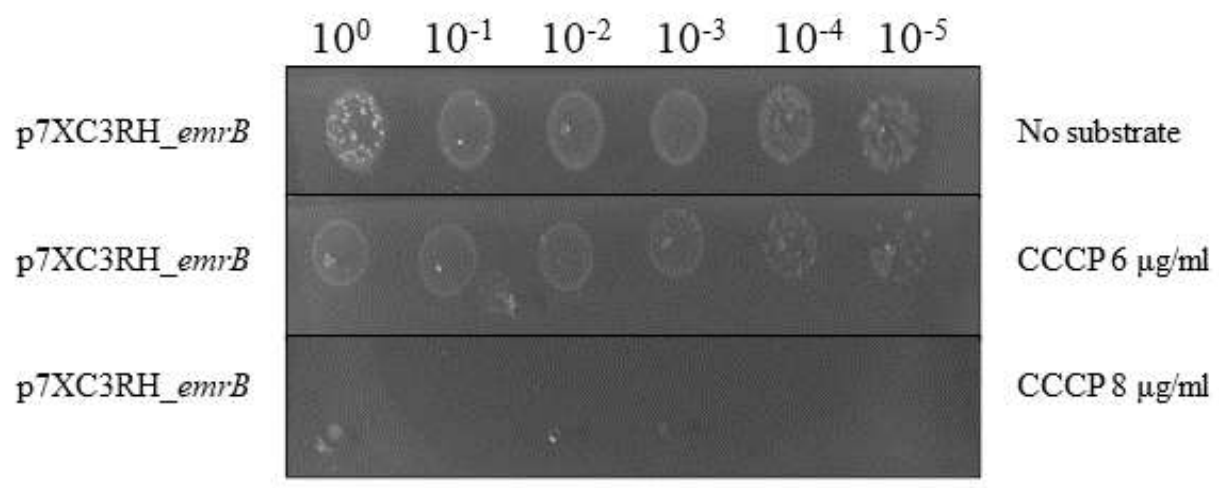

Figure 79. Drug susceptibility test of $E$. coli BW25113(DE3) $\triangle a c r A B$ harbouring p7XC3RH_emrB. Cells expressing the RND component of the pump could not survive at $8 \mu \mathrm{g} / \mathrm{ml}$ of CCCP. Cells were induced with $20 \mu \mathrm{M}$ IPTG. Culture dilution of cells with OD $600 \mathrm{~nm}$ of 1 to $10^{0}, 10^{-1}, 10^{-2}, 10^{-3}, 10^{-4}, 10^{-5}$. Results were scored after $18 \mathrm{~h}$ incubation at $37^{\circ} \mathrm{C}$. Images of the plates were produced with ImagerLAS4000.

Observation was made that when cells harboured only the gene for the inner membrane component of the pump, emrB-mRFP, showed higher susceptibility to the CCCP compared to the whole pump expressed. At the concentration of $8 \mu \mathrm{g} / \mathrm{ml}$ the cells expressing only emrB were completely suppressed in their growth (Figure 79). At the same concentration, cells expressing the pump were still growing until the last dilution step $\left(10^{-4}\right)$. The $\triangle a c r A B$ cells complemented with pET24a_pUC19 (Figure 78) or p7XC3RH_emrB (Figure 79) show comparable susceptibility profiles.

The complete inhibition of growth of the cells expressing the empty vectors was observed at $9 \mu \mathrm{g} / \mathrm{ml}$. At the same concentration, cells that contain empty vectors, but do not have the deletion of $a c r A B$, wild type BW25113, are still growing until the second dilution step $\left(10^{-2}\right)$. The cells that harbour the deletion of $a c r A B$ but overexpress the pump EmrAB-TolC, with $20 \mu \mathrm{M}$ IPTG as an inducer, are showing growth until the third dilution step. These observations were reproducible; the phenotypes of three individually picked clones are shown on Figure 80. 


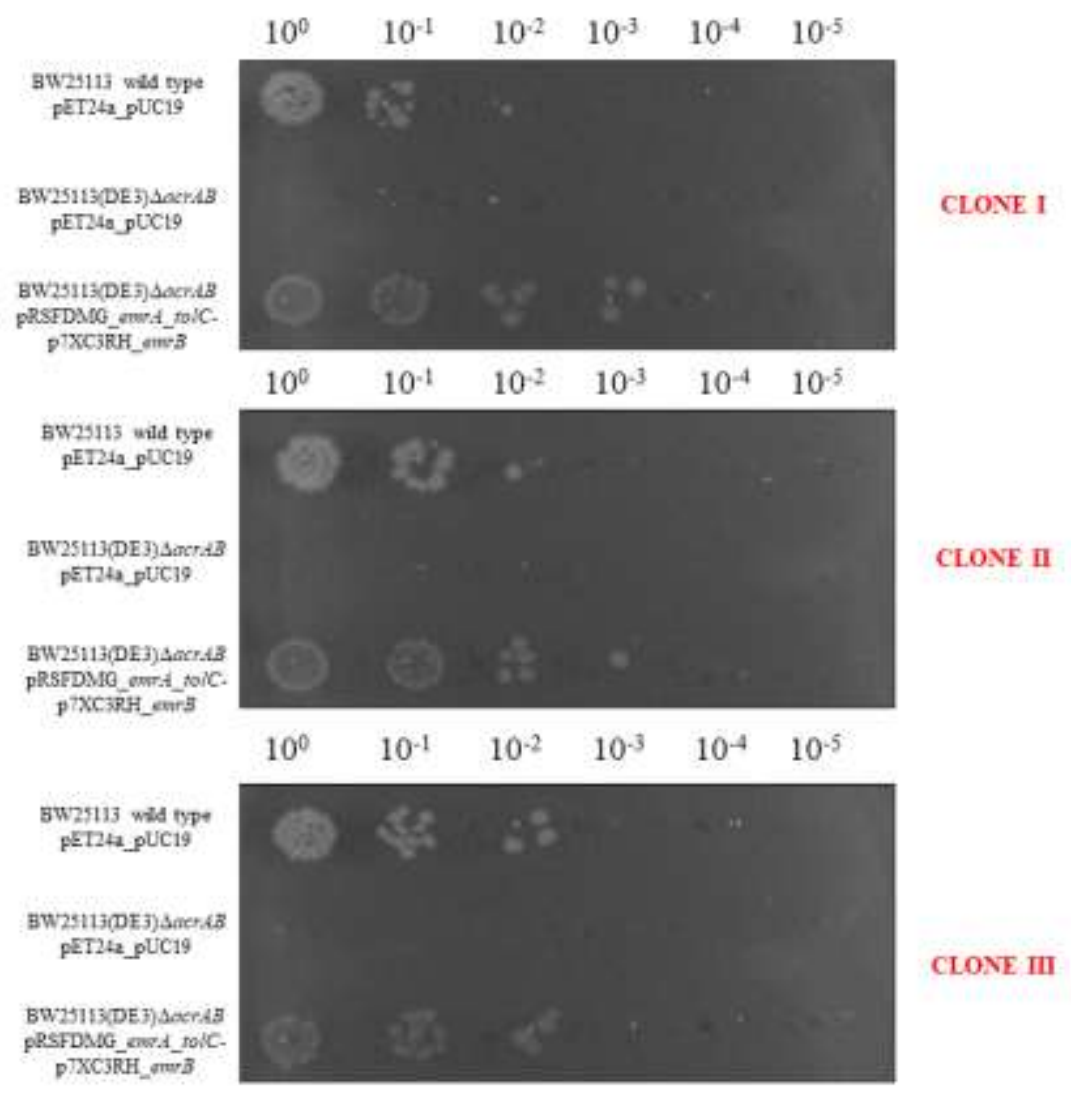

Figure 80. Drug susceptibility test of $E$. coli strains harbouring pRSFDMG_emrA_tolC and p7XC3RH_emrB. Empty vectors that harbour the same resistance cassettes were used, pET24a and pUC19 as a control. They were expressed in the wild type strain, BW25113 as well as in BW25113(DE3) $\triangle a c r A B$. Three individual clones were tested on the plates containing $9 \mu \mathrm{g} / \mathrm{ml}$ CCCP. Cells were induced with $20 \mu \mathrm{M}$ IPTG. Culture dilution of cells with $\mathrm{OD}_{600 \mathrm{~nm}}$ of 1 to $10^{0}, 10^{-1}, 10^{-2}, 10^{-3}, 10^{-4}, 10^{-5}$. Results were scored after $18 \mathrm{~h}$ incubation at $37^{\circ} \mathrm{C}$. Images of the plates were produced with ImagerLAS4000. Image adapted from (Yousefian et al., 2021), co-author agreement has been obtained.

\subsubsection{A drug susceptibility assay of $E$. coli expression strain, C41(DE3), harbouring genes expressing the MFS pump, EmrAB- TolC}

For the studies of the phenotypes on the solid medium the strain BW25113(DE3) $\triangle a c r A B$ is used. Since the expression of the EmrAB-TolC pump, for the EM studies (Yousefian et al., 2021) was performed in the E. coli C41(DE3) strain, an assay was performed with this strain. E. coli $\mathrm{C} 41(\mathrm{DE} 3)$ and $\mathrm{C} 41(\mathrm{DE} 3) \triangle a c r A B$ harbouring either empty vectors or genes encoding the red and green fluorescent fusions for the whole efflux pump were used in vivo on solid medium. 
As a control, E. coli wild type strain C41(DE3) expressing empty vectors, and E. coli C41(DE3) with a deletion of $a c r A B$ expressing either empty vectors or vectors encoding EmrAB-TolC were plated onto solid medium that does not contain the substrate CCCP. The cells were not inhibited to grow (Figure 81).

When CCCP is present in the medium, E. coli wild type and $\triangle a c r A B$ strains expressing empty vectors show inhibition of the growth; no growth was detected even on the first dilution step. E. coli $\mathrm{C} 41(\mathrm{DE} 3)$ harbouring deletion of $a c r A B$ and expressing the $e m r A$, emrB and $t o l C$, in the presence of only $3 \mu \mathrm{g} / \mathrm{ml} \mathrm{CCCP}$, shows significant inhibition of growth - cell growth was detectable until only the third dilution step.

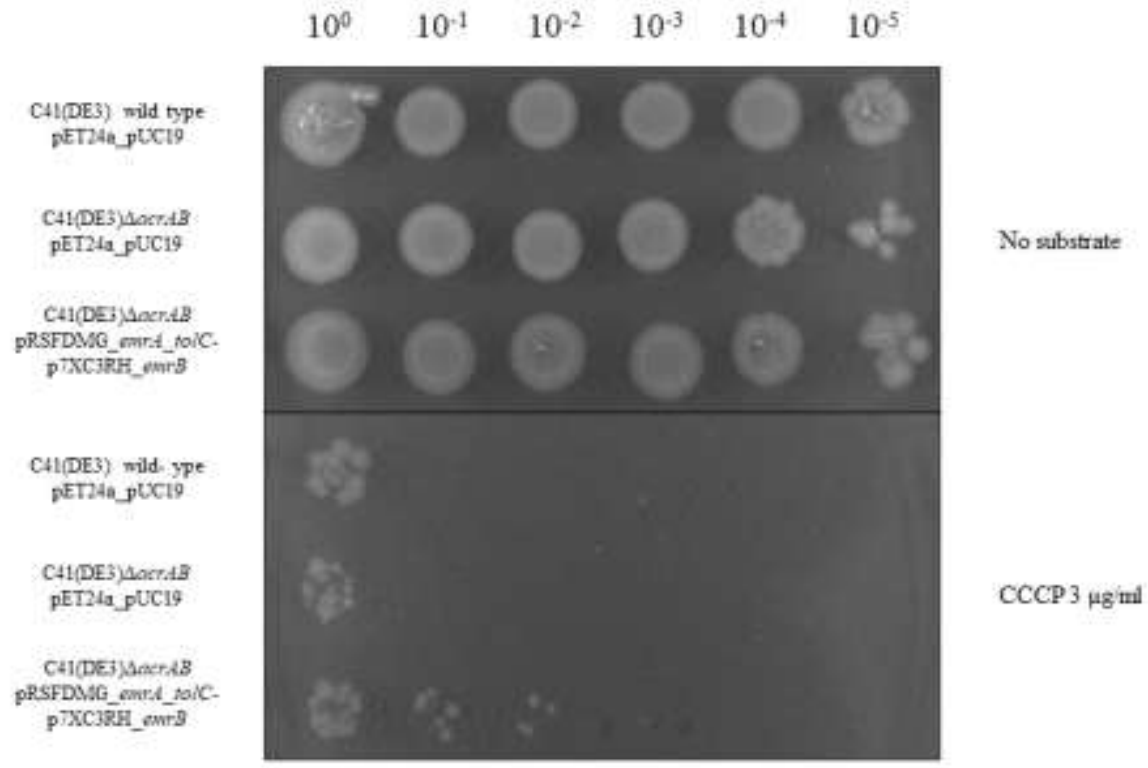

Figure 81. Drug susceptibility test of $E$. coli C41(DE3) wild type and C41(DE3) $\triangle a c r A B$ harbouring pRSFDMG_emrA_tolC and p7XC3RH_emrB. For the control empty vectors that harbour the same resistance cassettes were used, pET24a and pUC19. The inhibitory concentration tested and shown on the picture is $3 \mu \mathrm{g} / \mathrm{ml}$ of CCCP. Cells were induced with $20 \mu \mathrm{M}$ IPTG. Culture dilution of cells with $\mathrm{OD}_{600 \mathrm{~nm}}$ of 1 to $10^{0}, 10^{-1}, 10^{-2}, 10^{-3}, 10^{-4}, 10^{-5}$. Results were scored after $18 \mathrm{~h}$ incubation at $37^{\circ} \mathrm{C}$. Images of the plates were produced with ImagerLAS4000.

When the same constructs were tested at different concentrations of the inducer IPTG in the presence of the $2.5 \mu \mathrm{M}$ of CCCP, the observed behaviour was that the higher the concentration of the inducer, the better the cell growth (Figure 82). 


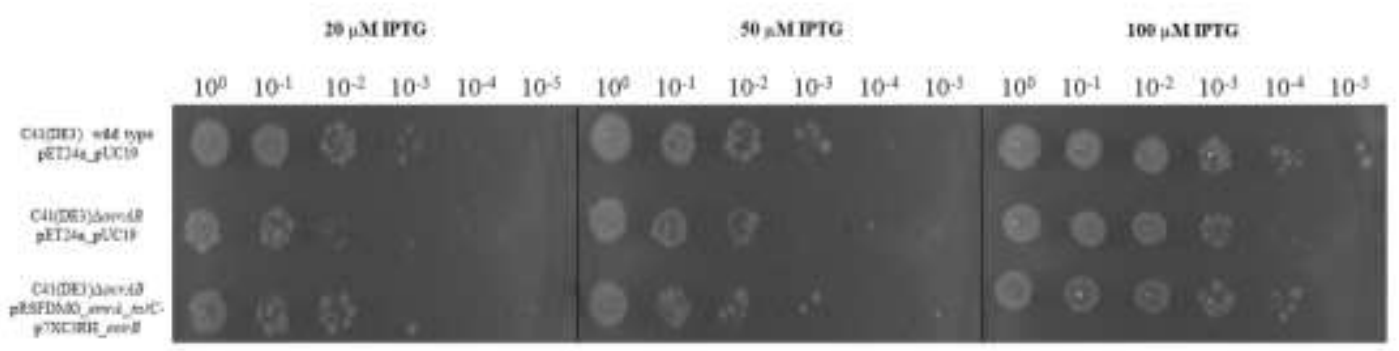

Figure 82. Drug susceptibility test of $E$. coli C41(DE3) wild type and C41(DE3) $\triangle a c r A B$ harbouring pRSFDMG_emrA_tolC and p7XC3RH_emrB. For the control empty vectors that harbour the same resistance cassettes were used, pET24a and pUC19. The inhibitory concentration tested and shown on the picture is $2.5 \mu \mathrm{g} / \mathrm{ml}$ of CCCP. Three different concentrations of the inducer, IPTG $(20,50$ or $100 \mu \mathrm{M})$ were used for testing of the expression level of the genes in comparison to the susceptibility. Culture dilution of cells with $\mathrm{OD}_{600 \mathrm{~nm}}$ of 1 to $10^{0}, 10^{-1}, 10^{-2}, 10^{-3}, 10^{-4}, 10^{-5}$. Results were scored after $18 \mathrm{~h}$ incubation at $37^{\circ} \mathrm{C}$. Images of the plates were produced with ImagerLAS4000.

\subsection{Discussion}

\subsubsection{E. coli strains show intrinsic resistance to CCCP}

The in vivo characterisation of the efflux pump, EmrAB-TolC, was conducted in the E. coli strain BW25113(DE3) $\triangle a c r A B$. For the control, the wild type strain BW25113 was used. Additionally, C41(DE3) $\triangle a c r A B$ strain was used with the respective control, C41(DE3) wild type.

When E. coli BW25113 cells lacking the $\operatorname{acr} A B$ genes were used, it resulted in increased susceptibilities to CCCP, in comparison to the wild type strain. Until the concentration of $5 \mu \mathrm{g} / \mathrm{ml}$, both strains are not inhibited to grow. From $8 \mu \mathrm{g} / \mathrm{ml}$, both strains show restriction in growth. Already at $10 \mu \mathrm{g} / \mathrm{ml}$ of $\mathrm{CCCP}$ in the solid medium, cells of the deletion strain stopped growing in the dilution steps, while the wild type was growing until the second dilution step (Figure 77). These observations go in contrast with the research of Sulavik et al in 2001, where the E. coli strain W3110 was used (Sulavik et al., 2001). In that study the deletions in $\operatorname{acr} A B$ did not result in an increased susceptibility to CCCP. Presumably the PDA assay from this study has a higher sensitivity in comparison to the Minimal Inhibitory Concentration assay (MIC) done by Sulavik et al in 2001 (Sulavik et al., 2001). 


\subsubsection{Cells expressing the MFS component show more susceptibility towards CCCP compared to cells expressing the whole pump}

When cells of the E. coli strain BW25113(DE3) $\triangle a c r A B$ overexpressing the efflux pump EmrAB-TolC were subjected to the phenotypic screening on the plates of the solid medium containing CCCP, a clear decrease in susceptibility was observable. At the concentration of CCCP of $6 \mu \mathrm{g} / \mathrm{ml}$, the cells expressing the efflux pump were growing to the fifth dilution step without repression. At the same concentration, cells harbouring empty plasmids showed initial inhibition of the growth at the fifth dilution step. On the control plate, with CCCP in the medium present, both constructs are growing without hindrance. When the CCCP concentration was increased to $8 \mu \mathrm{g} / \mathrm{ml}$, the cells with the empty plasmids, were inhibited to grow in the dilution series, while the cells with pump expressed, are growing until the second last dilution step, until $10^{-4}$ (Figure 78).

When the cells are expressing only the MFS component of the pump, emrB, they are more susceptible towards CCCP (Figure 79) and at the concentration of $8 \mu \mathrm{g} / \mathrm{ml}$ a total inhibition of the growth is observed. The cells expressing the whole pump were growing at the same concentration of CCCP until the second last dilution step. E. coli does not express emrA and emrB in standard conditions, it seems that presence of CCCP, induces the overexpression of this components of the efflux system EmrAB-TolC (O Lomovskaya et al., 1995).

\subsubsection{E coli $\mathrm{C41}(\mathrm{DE} 3)$ is not giving the clear phenotype picture}

The strain C41(DE3) was derived from BL21(DE3) (Miroux \& Walker, 1996). Comparative genomics and experimental evolution of E. coli strains BL21(DE3), C41(DE3) and C43(DE3) showed that hotspots for the mutations that inhibit cell death related to the expression of toxic recombinant proteins are located in the lacUV5 promoter of the DE3 region and in the lacI gene of the lac operon. The mutations in the lacUV5 promoter of the DE3 region are result of homologous recombination with the similar lac promoter in 
the lac operon (Wagner et al., 2008)(Kwon et al., 2015). Even though C41(DE3) strain has shown to be the best choice for the expression of EmrAB-TolC (Yousefian et al., 2021), it does not show the best qualities for the phenotypic studies. At the IPTG concentration of $20 \mu \mathrm{M}$, which was used in all previous experiments, C41(DE3) $\triangle a c r A B$ harbouring emrA, emrB and tolC on a plasmid, does not show a bigger difference in growth in comparison to the same strain harbouring empty vectors (the difference is in one dilution step). When IPTG concentration is increased $(50 \mu \mathrm{M}$ and $100 \mu \mathrm{M})$, the cells could grow more, until second or fourth dilution step, respectively, since the higher level of IPTG induces more expression (Figure 76). When the concentration of CCCP was increased (from $2.5 \mu \mathrm{g} / \mathrm{ml}$ to $3 \mu \mathrm{g} / \mathrm{ml}$ ), there was one dilution step increment of the cell growth in comparison to the empty vector, indicating that the efflux pump could still expel the compound. However, the most prominent phenotype was observed when the BW25113(DE3) $\triangle a c r A B$ strain was used and more significant difference between the empty vector and the efflux pump was observed. 


\section{Chapter VII: Conclusion and future perspectives}

Investigating the mechanisms of antibiotic resistance in bacteria is an important task. That said, the study of antibiotic and metal resistance mediated by efflux pumps is opening a window towards investigation of the systems that help Gram-negative bacteria to survive. $E$. coli efflux pumps belong to several different families of extruders, using different energy sources for activity and broadening the polyspecificity of bacterial resistance towards toxins. Extrusion of toxins happens from the cytoplasm and/or from the periplasm, while multicomponent efflux pumps, being the ones that span the whole Gram-negative bacterial envelope, help to direct the extrusion of compounds across the outer membrane.

In order to examine the assembly and drug transport properties of the Resistance Nodulation and cell Division (RND)-type efflux pump AcrAB-TolC, this work focused on establishing an in vitro assay for investigation of the conditions for the in vitro pump assembly and activity. When the RND component of the pump, AcrB, was reconstituted in the LUVs, it has been shown that $\mathrm{H}^{+}$is transported inside the proteoliposomes when a $\Delta \mathrm{pH}$ was applied to the system. Proton influx under the same conditions with proteoliposomes containing the inactive D407N, proton relay variant, or liposomes without reconstituted protein was negligible. This indicates that $\mathrm{AcrB}$ in the proteoliposomes transports $\mathrm{H}^{+}$across the membrane.

The role of the second component of the AcrAB-TolC system, the membrane fusion protein (MFP) AcrA, is still elusive. In this work, AcrA did not show further stimulation of the AcrB-mediated $\mathrm{H}^{+}$-influx. Using a different reconstitution setup, Zgurskaya et al. in 1999 conducted experiments in which AcrA enhances the AcrB-mediated substrate transport. In the homolog MexAB-OprM system from Pseudomonas aeruginosa, Verchère et al. in 2015 showed that the MFP MexA enhanced the activity of MexB (H. I. Zgurskaya \& Nikaido, 1999)(Verchère et al., 2015).

An in vitro assay was established for the investigation of the complex assembly of AcrABTolC. The strong, non-covalent, streptavidin-biotin bond was used in order to specifically address complex formation between AcrAB and TolC reconstituted in two different proteoliposomal batches. One of the batches contained biotinylated lipids and pull down with streptavidin coated magnetic beads indicated a co-precipitation of the non-biotinylated proteoliposomes, suggesting interaction of the AcrAB and TolC pump components. This 
interaction was observed under in the presence or absence of taurocholate, a substrate of the pump and/or by application of a $\Delta \mathrm{pH}$ across the membranes. When no $\mathrm{pH}$ gradient was applied, nor substrate or inhibitor was present, in vitro complex formation was still possible, although after a longer incubation time. Since AcrAB and TolC proteoliposomes are able to make contact in vitro, substrate transport assays using the tripartite setup are feasible. In this work, the interaction of AcrB containing proteoliposomes with TolC containing liposomes was observed. Further controls including non-cognate partners (e.g., TolC and CopA) will have to be conducted to further investigate this controversial observation.

Since the AcrAB-TolC system transports drugs which are considerable hydrophobic and spontaneously cross the membrane, another tripartite RND-system was taken to monitor substrate transport. The CusCBA system, where CusA is the AcrB homolog, transports $\mathrm{Ag}^{+}$ or $\mathrm{Cu}^{+}$in antiport with protons. These heavy metal ions can be detected using the fluorescence quenching of Phen Green SK (PGSK) upon binding. Previous work by Long et al in 2010 indicated the transport of $\mathrm{Ag}^{+}$into CusA-containing proteoliposomes under application of an outwardly-directed $\Delta \mathrm{pH}$, indicating sequestering of the $\mathrm{Ag}^{+}$ions at the cytoplasmic side of the CusA protein (Long et al., 2010). However, in vivo, monovalent heavy metal ions are also sequestered from the periplasm. In this work, several different conditions were used to trigger the inside-out or right-side out CusA protein in the proteoliposomal membrane. One experiment showed strong quenching of PGSK indicating transport of silver ions into the proteoliposomes. This condition included an inwardly directed $\Delta \mathrm{pH}$ and a $\Delta \psi$ (negative inside) imposed across the membrane. Under similar conditions, the acidification of the CusA containing proteoliposomes was tested and indicated strong quenching of the $\mathrm{H}^{+}$-sensitive dye 9-Amino-6-chloro-2-methoxyacridine (ACMA). This might indicate that the imposed $\Delta \psi$ (negative inside) is converted into an inverse $\Delta \mathrm{pH}$ (acidic inside) by diffusion of $\mathrm{H}^{+}$across the membrane, driving the antiport $\left(\mathrm{H}^{+}\right.$out, $\mathrm{Ag}^{+}$in). Further controls with the inactive CusA variant D405N have to be done to validate this observation.

In the last part of the thesis, I characterized the effect of the presence of the MFS-type EmrAB-TolC toward the susceptibility of carbonyl - cyanide m-chlorophenylhydrazone (CCCP) in E. coli. The in vivo experiments done in this study clearly showed the lower susceptibility of E. coli cells that overexpress this efflux pump towards CCCP. This activity needed all three components of the system, since the expression of EmrB alone did not affect the CCCP susceptibility. Moreover, the absence of the RND and MFP components AcrA and 
AcrB also affected CCCP-susceptibility; cells were more sensitive as compared to cells harbouring the AcrAB-TolC pump.

This work presents a foundation for future studies of multicomponent efflux pumps. Characterization of AcrAB-TolC and CusA as well as CopA via an in vitro assay, gives a basis for transport studies of toxins such as antibiotics, dyes, heavy metal ions, etc. Complex formation studies can be continued in the direction of the characterization of assemblydisassembly kinetics. Even though the usage of fluorescent dyes in a standard spectrophotometer is a useful tool to monitor the $\mathrm{H}^{+}$or substrate fluxes across the membrane, stopped-flow fluorescence quenching will allow for observing fast kinetic processes. For some of the AcrB substrates, radioactively labelled substrates are available which might be used in the future studies for discontinuous, but more quantitative analysis of the transport activity. For the investigation of the EmrAB-TolC pump, the residues involved in the binding and release of the substrates and the H+-coupling ion are still unknown, so that one of the first steps would be to conduct mutational analysis to identify the substrate binding site and proton relay network. Moreover, for the MFS-type tripartite EmrAB-TolC, only a lowresolution negative stain map is available (Yousefian et al., 2021), so here a high-resolution structure will help to extend toward the identification of the drug binding site and eventually for the design of new potent pump inhibitors.

Fighting microbes that are resistant to almost all drugs available on the market nowadays is a great challenge. Every small step in the understanding of the mechanisms deployed by bacteria to expel toxic compounds out of the cell, is a big step towards saving lives. 


\section{$\underline{\text { References }}$}

Abeyrathna, N., Abeyrathna, S., Morgan, M. T., Fahrni, C. J., \& Meloni, G. (2020). Transmembrane $\mathrm{Cu}(<\mathrm{scp}>\mathrm{i}</ \mathrm{scp}>$ ) P-type ATPase pumps are electrogenic uniporters. Dalton Transactions, 49(45), 16082-16094. https://doi.org/10.1039/D0DT01380C

Adam, Y., Tayer, N., Rotem, D., Schreiber, G., \& Schuldiner, S. (2007). The fast release of sticky protons: Kinetics of substrate binding and proton release in a multidrug transporter. Proceedings of the National Academy of Sciences, 104(46), 17989-17994. https://doi.org/10.1073/pnas.0704425104

Adzitey, F. (2015). Antibiotic Classes and Antibiotic Susceptibility of Bacterial Isolates from Selected Poultry; A Mini Review. World $s$ Veterinary Journal, 6(1), 36. https://doi.org/10.5455/wvj.20150853

Aires, J. R., \& Nikaido, H. (2005). Aminoglycosides Are Captured from both Periplasm and Cytoplasm by the AcrD Multidrug Efflux Transporter of Escherichia coli. Journal of Bacteriology, 187(6), 1923-1929. https://doi.org/10.1128/JB.187.6.1923-1929.2005

Altmann, S. W. (2004). Niemann-Pick C1 Like 1 Protein Is Critical for Intestinal Cholesterol Absorption. Science, 303(5661), 1201-1204. https://doi.org/10.1126/science.1093131

Andersen, C., Koronakis, E., Bokma, E., Eswaran, J., Humphreys, D., Hughes, C., \& Koronakis, V. (2002). Transition to the open state of the TolC periplasmic tunnel entrance. Proceedings of the National Academy of Sciences, 99(17), 11103-11108. https://doi.org/10.1073/pnas.162039399

Arenz, S., \& Wilson, D. N. (2016). Bacterial Protein Synthesis as a Target for Antibiotic Inhibition. Cold Spring Harbor Perspectives in Medicine, 6(9), a025361. https://doi.org/10.1101/cshperspect.a025361

Arthur, J. C., \& Jobin, C. (2013). The complex interplay between inflammation, the microbiota and colorectal cancer. Gut Microbes, 4(3). https://doi.org/10.4161/gmic. 24220

Arthur, J. C., Perez-Chanona, E., Mühlbauer, M., Tomkovich, S., Uronis, J. M., Fan, T.-J., Campbell, B. J., Abujamel, T., Dogan, B., Rogers, A. B., Rhodes, J. M., Stintzi, A., Simpson, K. W., Hansen, J. J., Keku, T. O., Fodor, A. A., \& Jobin, C. (2012). Intestinal Inflammation Targets Cancer-Inducing Activity of the Microbiota. Science, 338(6103). https://doi.org/10.1126/science. 1224820

Atzori, A., Malloci, G., Cardamone, F., Bosin, A., Vargiu, A. V., \& Ruggerone, P. (2020). Molecular Interactions of Carbapenem Antibiotics with the Multidrug Efflux Transporter AcrB of Escherichia coli. International Journal of Molecular Sciences, 21(3), 860. https://doi.org/10.3390/ijms21030860

Bakker-Woudenberg, I. A. J. M., ten Kate, M. T., Guo, L., Working, P., \& Mouton, J. W. (2001). Improved Efficacy of Ciprofloxacin Administered in Polyethylene GlycolCoated Liposomes for Treatment of Klebsiella pneumoniae Pneumonia in Rats. Antimicrobial Agents and Chemotherapy, 45(5), 1487-1492. https://doi.org/10.1128/AAC.45.5.1487-1492.2001

Bakker-Woudenberg, I. A. J. M., ten Kate, M. T., Guo, L., Working, P., \& Mouton, J. W. (2002). Ciprofloxacin in polyethylene glycol-coated liposomes: efficacy in rat models of 
acute or chronic Pseudomonas aeruginosa infection. Antimicrobial Agents and Chemotherapy, 46(8), 2575-2581. https://doi.org/10.1128/aac.46.8.2575-2581.2002

Bangham, A. D., Hill, M. W., \& Miller, N. G. A. (1974). Preparation and Use of Liposomes as Models of Biological Membranes. In Methods in Membrane Biology (pp. 1-68). Springer US. https://doi.org/10.1007/978-1-4615-7422-4_1

Bangham, A. D., Standish, M. M., \& Weissmann, G. (1965). The action of steroids and streptolysin $\mathrm{S}$ on the permeability of phospholipid structures to cations. Journal of Molecular Biology, 13(1), 253-IN28. https://doi.org/10.1016/S0022-2836(65)80094-8

Bas, D. C., Rogers, D. M., \& Jensen, J. H. (2008). Very fast prediction and rationalization of $\mathrm{pKa}$ values for protein-ligand complexes. Proteins: Structure, Function, and Bioinformatics, 73(3), 765-783. https://doi.org/10.1002/prot.22102

Bassetti, M., Poulakou, G., Ruppe, E., Bouza, E., Van Hal, S. J., \& Brink, A. (2017). Antimicrobial resistance in the next 30 years, humankind, bugs and drugs: a visionary approach. Intensive Care Medicine, 43(10), 1464-1475. https://doi.org/10.1007/s00134$017-4878-\mathrm{x}$

Benz, R., Maier, E., \& Gentschev, I. (1993). TolC of Escherichia coli Functions as an Outer Membrane Channel. Zentralblatt Für Bakteriologie, 278(2-3), 187-196. https://doi.org/10.1016/S0934-8840(11)80836-4

BioRender https://app.biorender.com/

Blair, J. M. A., Webber, M. A., Baylay, A. J., Ogbolu, D. O., \& Piddock, L. J. V. (2015). Molecular mechanisms of antibiotic resistance. Nature Reviews Microbiology, 13(1), 42-51. https://doi.org/10.1038/nrmicro3380

Blount, Z. D. (2015). The unexhausted potential of E. coli. ELife, 4. https://doi.org/10.7554/eLife.05826

Bohnert, J. A., Schuster, S., Seeger, M. A., Fähnrich, E., Pos, K. M., \& Kern, W. V. (2008). Site-Directed Mutagenesis Reveals Putative Substrate Binding Residues in the Escherichia coli RND Efflux Pump AcrB. Journal of Bacteriology, 190(24), 8225-8229. https://doi.org/10.1128/JB.00912-08

Bos, E. R., Jamison, D. T., Bainga, F., Feacham, R. G. A., Makgoba, M., Hofman, K. J., \& Rogo, hama O. (Eds.). (2006). Disease and Mortality in Sub-Saharan Africa. The World Bank. https://doi.org/10.1596/978-0-8213-6397-3

Bovier, P. A. (2008). Epaxal ${ }^{\circledR}$ : a virosomal vaccine to prevent hepatitis A infection. Expert Review of Vaccines, 7(8), 1141-1150. https://doi.org/10.1586/14760584.7.8.1141

Boyer, P. D. (1997). THE ATP SYNTHASE-A SPLENDID MOLECULAR MACHINE. $\begin{array}{llll}\text { Annual Review } & \text { of }\end{array}$ https://doi.org/10.1146/annurev.biochem.66.1.717

Brown, E. D., Vivas, E. I., Walsh, C. T., \& Kolter, R. (1995). MurA (MurZ), the enzyme that catalyzes the first committed step in peptidoglycan biosynthesis, is essential in Escherichia coli. Journal of Bacteriology, 177(14), 4194-4197. https://doi.org/10.1128/JB.177.14.4194-4197.1995

Bulbake, U., Doppalapudi, S., Kommineni, N., \& Khan, W. (2017). Liposomal Formulations in Clinical Use: An Updated Review. Pharmaceutics, 9(4), 12. 
https://doi.org/10.3390/pharmaceutics9020012

Campanhã, M. T. N., Mamizuka, E. M., \& Carmona-Ribeiro, A. M. (1999). Interactions between cationic liposomes and bacteria: the physical-chemistry of the bactericidal action. Journal of Lipid Research, 40(8), 1495-1500. https://doi.org/10.1016/S00222275(20)33392-7

Cantón, R., González-Alba, J. M., \& Galán, J. C. (2012). CTX-M Enzymes: Origin and Diffusion. Frontiers in Microbiology, 3. https://doi.org/10.3389/fmicb.2012.00110

Casadaban, M. J., \& Cohen, S. N. (1980). Analysis of gene control signals by DNA fusion and cloning in Escherichia coli. Journal of Molecular Biology, 138(2), 179-207. https://doi.org/10.1016/0022-2836(80)90283-1

Cha, H., Müller, R. T., \& Pos, K. M. (2014). Switch-Loop Flexibility Affects Transport of Large Drugs by the Promiscuous AcrB Multidrug Efflux Transporter. Antimicrobial Agents and Chemotherapy, 58(8), 4767-4772. https://doi.org/10.1128/AAC.02733-13

Chacon, K. N., Mealman, T. D., McEvoy, M. M., \& Blackburn, N. J. (2014). Tracking metal ions through a $\mathrm{Cu} / \mathrm{Ag}$ efflux pump assigns the functional roles of the periplasmic proteins. Proceedings of the National Academy of Sciences, 111(43), 15373-15378. https://doi.org/10.1073/pnas.1411475111

Dadgostar, P. (2019). Antimicrobial Resistance: Implications and Costs. Infection and Drug Resistance, Volume 12, 3903-3910. https://doi.org/10.2147/IDR.S234610

Daraee, H., Etemadi, A., Kouhi, M., Alimirzalu, S., \& Akbarzadeh, A. (2016). Application of liposomes in medicine and drug delivery. Artificial Cells, Nanomedicine, and Biotechnology, 44(1), 381-391. https://doi.org/10.3109/21691401.2014.953633

Datsenko, K. A., \& Wanner, B. L. (2000). One-step inactivation of chromosomal genes in Escherichia coli K-12 using PCR products. Proceedings of the National Academy of Sciences, 97(12), 6640-6645. https://doi.org/10.1073/pnas.120163297

Daury, L., Orange, F., Taveau, J.-C., Verchère, A., Monlezun, L., Gounou, C., Marreddy, R. K. R., Picard, M., Broutin, I., Pos, K. M., \& Lambert, O. (2016). Tripartite assembly of RND multidrug efflux pumps. Nature Communications, 7(1), 10731. https://doi.org/10.1038/ncomms10731

de Freitas, C. F., Calori, I. R., Tessaro, A. L., Caetano, W., \& Hioka, N. (2019). Rapid formation of Small Unilamellar Vesicles (SUV) through low-frequency sonication: An innovative approach. Colloids and Surfaces B: Biointerfaces, 181, 837-844. https://doi.org/10.1016/j.colsurfb.2019.06.027

de Kraker, M. E. A., Stewardson, A. J., \& Harbarth, S. (2016). Will 10 Million People Die a Year due to Antimicrobial Resistance by 2050? PLOS Medicine, 13(11), e1002184. https://doi.org/10.1371/journal.pmed.1002184

de Louvois, J. (1994). Acute bacterial meningitis in the newborn. Journal of Antimicrobial Chemotherapy, 34(suppl A). https://doi.org/10.1093/jac/34.suppl_A.61

Dezi, M., Di Cicco, A., Bassereau, P., \& Levy, D. (2013). Detergent-mediated incorporation of transmembrane proteins in giant unilamellar vesicles with controlled physiological contents. Proceedings of the National Academy of Sciences, 110(18), 7276-7281. https://doi.org/10.1073/pnas.1303857110 
Doi, Y., Wachino, J., \& Arakawa, Y. (2016). Aminoglycoside Resistance. Infectious Disease Clinics of North America, 30(2), 523-537. https://doi.org/10.1016/j.idc.2016.02.011

Du, D., \& Luisi, B. F. (2015). Bacterial multidrug efflux pumps: ... and some suggestions on how they might work. The Biochemist, 37(3), 22-27. https://doi.org/10.1042/BIO03703022

Du, D., van Veen, H. W., \& Luisi, B. F. (2015). Assembly and operation of bacterial tripartite multidrug efflux pumps. Trends in Microbiology, 23(5), 311-319. https://doi.org/10.1016/j.tim.2015.01.010

Du, D., Wang-Kan, X., Neuberger, A., van Veen, H. W., Pos, K. M., Piddock, L. J. V., \& Luisi, B. F. (2018). Multidrug efflux pumps: structure, function and regulation. Nature Reviews Microbiology, 16(9), 523-539. https://doi.org/10.1038/s41579-018-0048-6

Du, D., Wang, Z., James, N. R., Voss, J. E., Klimont, E., Ohene-Agyei, T., Venter, H., Chiu, W., \& Luisi, B. F. (2014). Structure of the AcrAB-TolC multidrug efflux pump. Nature, 509(7501), 512-515. https://doi.org/10.1038/nature13205

ECDC. (2018). 33000 people die every year due to infections with antibiotic-resistant bacteria. Https://Www.Ecdc.Europa.Eu/. https://www.ecdc.europa.eu/en/newsevents/33000-people-die-every-year-due-infections-antibiotic-resistant-bacteria

Eicher, T., Cha, H. -j., Seeger, M. A., Brandstatter, L., El-Delik, J., Bohnert, J. A., Kern, W. V., Verrey, F., Grutter, M. G., Diederichs, K., \& Pos, K. M. (2012). Transport of drugs by the multidrug transporter AcrB involves an access and a deep binding pocket that are separated by a switch-loop. Proceedings of the National Academy of Sciences, 109(15), 5687-5692. https://doi.org/10.1073/pnas.1114944109

Eicher, Thomas, Seeger, M. A., Anselmi, C., Zhou, W., Brandstätter, L., Verrey, F., Diederichs, K., Faraldo-Gómez, J. D., \& Pos, K. M. (2014). Coupling of remote alternating-access transport mechanisms for protons and substrates in the multidrug efflux pump AcrB. ELife, 3. https://doi.org/10.7554/eLife.03145

Evans, D. J., \& Evans, D. G. (1996). Escherichia Coli in Diarrheal Disease. In Medical Microbiology.

Ewers, C., Bethe, A., Semmler, T., Guenther, S., \& Wieler, L. H. (2012). Extended-spectrum $\beta$-lactamase-producing and AmpC-producing Escherichia coli from livestock and companion animals, and their putative impact on public health: a global perspective. Clinical Microbiology and Infection, 18(7), 646-655. https://doi.org/10.1111/j.14690691.2012.03850.x

Fàbrega, A., Madurga, S., Giralt, E., \& Vila, J. (2009). Mechanism of action of and resistance to quinolones. Microbial Biotechnology, 2(1), 40-61. https://doi.org/10.1111/j.17517915.2008.00063.x

Fan, B., \& Rosen, B. P. (2002). Biochemical Characterization of CopA, the Escherichia coli $\mathrm{Cu}(\mathrm{I})$-translocating P-type ATPase. Journal of Biological Chemistry, 277(49), 4698746992. https://doi.org/10.1074/jbc.M208490200

Fischer, J., Rodriguez, I., Schmoger, S., Friese, A., Roesler, U., Helmuth, R., \& Guerra, B. (2012). Escherichia coli producing VIM-1 carbapenemase isolated on a pig farm. Journal of Antimicrobial Chemotherapy, 67(7), 1793-1795. https://doi.org/10.1093/jac/dks108 
Fitzpatrick, A. W. P., Llabrés, S., Neuberger, A., Blaza, J. N., Bai, X.-C., Okada, U., Murakami, S., van Veen, H. W., Zachariae, U., Scheres, S. H. W., Luisi, B. F., \& Du, D. (2017). Structure of the MacAB-TolC ABC-type tripartite multidrug efflux pump. Nature Microbiology, 2(7), 17070. https://doi.org/10.1038/nmicrobiol.2017.70

Fourmy, D., Recht, M. I., \& Puglisi, J. D. (1998). Binding of neomycin-class aminoglycoside antibiotics to the A-site of 16 s rRNA. Journal of Molecular Biology, 277(2), 347-362. https://doi.org/10.1006/jmbi.1997.1552

Franke, S., Grass, G., Rensing, C., \& Nies, D. H. (2003). Molecular analysis of the coppertransporting efflux system CusCFBA of Escherichia coli. Journal of Bacteriology, 185(13), 3804-3812. https://doi.org/10.1128/jb.185.13.3804-3812.2003

Furukawa, H., Tsay, J. T., Jackowski, S., Takamura, Y., \& Rock, C. O. (1993). Thiolactomycin resistance in Escherichia coli is associated with the multidrug resistance efflux pump encoded by emrAB. Journal of Bacteriology, 175(12), 3723-3729. https://doi.org/10.1128/JB.175.12.3723-3729.1993

Gabizon, A., Catane, R., Uziely, B., Kaufman, B., Safra, T., Cohen, R., Martin, F., Huang, A., \& Barenholz, Y. (1994). Prolonged circulation time and enhanced accumulation in malignant exudates of doxorubicin encapsulated in polyethylene-glycol coated liposomes. Cancer Research, 54(4), 987-992.

Garg, S. K., Singh, O., Juneja, D., Tyagi, N., Khurana, A. S., Qamra, A., Motlekar, S., \& Barkate, H. (2017). Resurgence of Polymyxin B for MDR/XDR Gram-Negative Infections: An Overview of Current Evidence. Critical Care Research and Practice, 2017, 1-10. https://doi.org/10.1155/2017/3635609

Geertsma, E. R., \& Dutzler, R. (2011). A Versatile and Efficient High-Throughput Cloning Tool for Structural Biology. Biochemistry, 50(15), 3272-3278. https://doi.org/10.1021/bi200178z

Geertsma, E. R., Nik Mahmood, N. A. B., Schuurman-Wolters, G. K., \& Poolman, B. (2008). Membrane reconstitution of $\mathrm{ABC}$ transporters and assays of translocator function. Nature Protocols, 3(2), 256-266. https://doi.org/10.1038/nprot.2007.519

Glantz, M. J., Jaeckle, K. A., Chamberlain, M. C., Phuphanich, S., Recht, L., Swinnen, L. J., Maria, B., LaFollette, S., Schumann, G. B., Cole, B. F., \& Howell, S. B. (1999). A randomized controlled trial comparing intrathecal sustained-release cytarabine (DepoCyt) to intrathecal methotrexate in patients with neoplastic meningitis from solid tumors. Clinical Cancer Research : An Official Journal of the American Association for Cancer Research, 5(11), 3394-3402.

Glavier, M., Puvanendran, D., Salvador, D., Decossas, M., Phan, G., Garnier, C., Frezza, E., Cece, Q., Schoehn, G., Picard, M., Taveau, J.-C., Daury, L., Broutin, I., \& Lambert, O. (2020). Antibiotic export by MexB multidrug efflux transporter is allosterically controlled by a MexA-OprM chaperone-like complex. Nature Communications, 11(1), 4948. https://doi.org/10.1038/s41467-020-18770-5

Gong, X., Qian, H., Zhou, X., Wu, J., Wan, T., Cao, P., Huang, W., Zhao, X., Wang, X., Wang, P., Shi, Y., Gao, G. F., Zhou, Q., \& Yan, N. (2016). Structural Insights into the Niemann-Pick C1 (NPC1)-Mediated Cholesterol Transfer and Ebola Infection. Cell, 165(6), 1467-1478. https://doi.org/10.1016/j.cell.2016.05.022

González, M., Argaraña, C. E., \& Fidelio, G. D. (1999). Extremely high thermal stability of 
streptavidin and avidin upon biotin binding. Biomolecular Engineering, 16(1-4), 67-72. https://doi.org/10.1016/S1050-3862(99)00041-8

Grenier, F., Matteau, D., Baby, V., \& Rodrigue, S. (2014). Complete Genome Sequence of Escherichia coli BW25113. Genome Announcements, 2(5). https://doi.org/10.1128/genomeA.01038-14

Griffey, R. H., Hofstadler, S. A., Sannes-Lowery, K. A., Ecker, D. J., \& Crooke, S. T. (1999). Determinants of aminoglycoside-binding specificity for rRNA by using mass spectrometry. Proceedings of the National Academy of Sciences, 96(18), 10129-10133. https://doi.org/10.1073/pnas.96.18.10129

Griffin, P. M., \& Tauxe, R. V. (1991). The Epidemiology of Infections Caused by Escherichia coli O157: H7, Other Enterohemorrhagic E. coli, and the Associated Hemolytic Uremic Syndrome. Epidemiologic Reviews, 13(1). https://doi.org/10.1093/oxfordjournals.epirev.a036079

Hansen, L. H., Johannesen, E., Burmølle, M., Sørensen, A. H., \& Sørensen, S. J. (2004). Plasmid-Encoded Multidrug Efflux Pump Conferring Resistance to Olaquindox in Escherichia coli. Antimicrobial Agents and Chemotherapy, 48(9), 3332-3337. https://doi.org/10.1128/AAC.48.9.3332-3337.2004

Hassan, K. A., Elbourne, L. D. H., Li, L., Gamage, H. K. A. H., Liu, Q., Jackson, S. M., Sharples, D., Kolstø, A.-B., Henderson, P. J. F., \& Paulsen, I. T. (2015). An ace up their sleeve: a transcriptomic approach exposes the AceI efflux protein of Acinetobacter baumannii and reveals the drug efflux potential hidden in many microbial pathogens. Frontiers in Microbiology, 6. https://doi.org/10.3389/fmicb.2015.00333

Hassan, K. A., Liu, Q., Henderson, P. J. F., \& Paulsen, I. T. (2015). Homologs of the Acinetobacter baumannii AceI Transporter Represent a New Family of Bacterial Multidrug Efflux Systems. MBio, 6(1). https://doi.org/10.1128/mBio.01982-14

Hassan, K. A., Naidu, V., Edgerton, J. R., Mettrick, K. A., Liu, Q., Fahmy, L., Li, L., Jackson, S. M., Ahmad, I., Sharples, D., Henderson, P. J. F., \& Paulsen, I. T. (2019). Short-chain diamines are the physiological substrates of PACE family efflux pumps. Proceedings of the National Academy of Sciences, 116(36), 18015-18020. https://doi.org/10.1073/pnas.1901591116

He, H., Lu, Y., Qi, J., Zhu, Q., Chen, Z., \& Wu, W. (2019). Adapting liposomes for oral drug delivery. Acta Pharmaceutica Sinica B, 9(1), 36-48. https://doi.org/10.1016/j.apsb.2018.06.005

Heimburg, T. (2009). Physical Properties of Biological Membranes.

Hendlin, D., Stapley, E. O., Jackson, M., Wallick, H., Miller, A. K., Wolf, F. J., Miller, T. W., Chaiet, L., Kahan, F. M., Foltz, E. L., Woodruff, H. B., Mata, J. M., Hernandez, S., \& Mochales, S. (1969). Phosphonomycin, a New Antibiotic Produced by Strains of Streptomyces. $\quad$ Science, 166(3901), $122-123$. https://doi.org/10.1126/science.166.3901.122

Heng, J., Zhao, Y., Liu, M., Liu, Y., Fan, J., Wang, X., Zhao, Y., \& Zhang, X. C. (2015). Substrate-bound structure of the E. coli multidrug resistance transporter MdfA. Cell Research, 25(9), 1060-1073. https://doi.org/10.1038/cr.2015.94

Heukeshoven, J., \& Dernick, R. (1985). Simplified method for silver staining of proteins in 
polyacrylamide gels and the mechanism of silver staining. Electrophoresis, 6(3), 103112. https://doi.org/10.1002/elps.1150060302

Hinchliffe, P., Greene, N. P., Paterson, N. G., Crow, A., Hughes, C., \& Koronakis, V. (2014). Structure of the periplasmic adaptor protein from a major facilitator superfamily (MFS) multidrug efflux pump. FEBS Letters, 588(17), 3147-3153. https://doi.org/10.1016/j.febslet.2014.06.055

Hofheinz, R.-D., Gnad-Vogt, S. U., Beyer, U., \& Hochhaus, A. (2005). Liposomal encapsulated anti-cancer drugs. Anti-Cancer Drugs, 16(7), 691-707. https://doi.org/10.1097/01.cad.0000167902.53039.5a

Honoré, N., Nicolas, M. H., \& Cole, S. T. (1986). Inducible cephalosporinase production in clinical isolates of Enterobacter cloacae is controlled by a regulatory gene that has been deleted from Escherichia coli. The EMBO Journal, 5(13), 3709-3714. https://doi.org/10.1002/j.1460-2075.1986.tb04704.x

Hooper, D. C. (1999). Mechanisms of fluoroquinolone resistance. Drug Resistance Updates, 2(1), 38-55. https://doi.org/10.1054/drup.1998.0068

Hope, M. J., Bally, M. B., Webb, G., \& Cullis, P. R. (1985). Production of large unilamellar vesicles by a rapid extrusion procedure. Characterization of size distribution, trapped volume and ability to maintain a membrane potential. Biochimica et Biophysica Acta (BBA) - Biomembranes, 812(1), 55-65. https://doi.org/10.1016/0005-2736(85)90521-8

Howarth, M., \& Ting, A. Y. (2008). Imaging proteins in live mammalian cells with biotin ligase and monovalent streptavidin. Nature Protocols, 3(3), 534-545. https://doi.org/10.1038/nprot.2008.20

Hudault, S. (2001). Escherichia coli strains colonising the gastrointestinal tract protect germfree mice against Salmonella typhimurium infection. Gut, 49(1). https://doi.org/10.1136/gut.49.1.47

Husain, F., Humbard, M., \& Misra, R. (2004). Interaction between the TolC and AcrA Proteins of a Multidrug Efflux System of Escherichia coli. Journal of Bacteriology, 186(24), 8533-8536. https://doi.org/10.1128/JB.186.24.8533-8536.2004

Jaurin, B., Grundström, T., \& Normark, S. (1982). Sequence elements determining ampC promoter strength in E. coli. The EMBO Journal, 1(7), 875-881. https://doi.org/10.1002/j.1460-2075.1982.tb01263.x

Johnson, J. R., \& Russo, T. A. (2002). Extraintestinal pathogenic Escherichia coli: "The other bad E coli." Journal of Laboratory and Clinical Medicine, 139(3). https://doi.org/10.1067/mlc.2002.121550

Juliano, R. L., \& Ling, V. (1976). A surface glycoprotein modulating drug permeability in Chinese hamster ovary cell mutants. Biochimica et Biophysica Acta (BBA) Biomembranes, 455(1), 152-162. https://doi.org/10.1016/0005-2736(76)90160-7

Kano, K., \& Fendler, J. H. (1978). Pyranine as a sensitive pH probe for liposome interiors and surfaces. $\mathrm{pH}$ gradients across phospholipid vesicles. Biochimica et Biophysica Acta (BBA) - Biomembranes, 509(2), 289-299. https://doi.org/10.1016/0005-2736(78)900482

Karami, N., Nowrouzian, F., Adlerberth, I., \& Wold, A. E. (2006). Tetracycline Resistance in Escherichia coli and Persistence in the Infantile Colonic Microbiota. Antimicrobial 
Agents and Chemotherapy, 50(1), 156-161. https://doi.org/10.1128/AAC.50.1.156161.2006

Kim, H., \& Ahner, B. A. (2006). Calibration of Phen Green ${ }^{\mathrm{TM}}$ for use as a Cu(I)-selective fluorescent indicator. Analytica Chimica Acta, 575(2), 223-229. https://doi.org/10.1016/j.aca.2006.05.089

Kinana, A. D., Vargiu, A. V., May, T., \& Nikaido, H. (2016). Aminoacyl $\beta$-naphthylamides as substrates and modulators of AcrB multidrug efflux pump. Proceedings of the National Academy of Sciences, 113(5), 1405-1410. https://doi.org/10.1073/pnas.1525143113

Klenotic, P. A., Moseng, M. A., Morgan, C. E., \& Yu, E. W. (2020). Structural and Functional Diversity of Resistance-Nodulation-Cell Division Transporters. Chemical Reviews. https://doi.org/10.1021/acs.chemrev.0c00621

Knol, J., Veenhoff, L., Liang, W.-J., Henderson, P. J. F., Leblanc, G., \& Poolman, B. (1996). Unidirectional Reconstitution into Detergent-destabilized Liposomes of the Purified Lactose Transport System of. Journal of Biological Chemistry, 271(26), 15358-15366. https://doi.org/10.1074/jbc.271.26.15358

Kobylka, J., Kuth, M. S., Müller, R. T., Geertsma, E. R., \& Pos, K. M. (2020). AcrB: a mean, keen, drug efflux machine. Annals of the New York Academy of Sciences, 1459(1), 3868. https://doi.org/10.1111/nyas.14239

Koronakis, V., Sharff, A., Koronakis, E., Luisi, B., \& Hughes, C. (2000). Crystal structure of the bacterial membrane protein TolC central to multidrug efflux and protein export. Nature, 405(6789), 914-919. https://doi.org/10.1038/35016007

Krogfelt, K. A., Cohen, P. S., \& Conway, T. (2004). The Life of Commensal Escherichia coli in the Mammalian Intestine. EcoSal Plus, 1(1). https://doi.org/10.1128/ecosalplus.8.3.1.2

Kwon, S.-K., Kim, S. K., Lee, D.-H., \& Kim, J. F. (2015). Comparative genomics and experimental evolution of Escherichia coli BL21(DE3) strains reveal the landscape of toxicity escape from membrane protein overproduction. Scientific Reports, 5(1), 16076. https://doi.org/10.1038/srep16076

LAEMMLI, U. K. (1970). Cleavage of Structural Proteins during the Assembly of the Head of Bacteriophage T4. Nature, 227(5259), 680-685. https://doi.org/10.1038/227680a0

Lambert, O., Levy, D., Ranck, J.-L., Leblanc, G., \& Rigaud, J.-L. (1998). A New “Gel-like” Phase in Dodecyl Maltoside-Lipid Mixtures: Implications in Solubilization and Reconstitution Studies. Biophysical Journal, 74(2), 918-930. https://doi.org/10.1016/S0006-3495(98)74015-9

Lewinson, O., Padan, E., \& Bibi, E. (2004). Alkalitolerance: A biological function for a multidrug transporter in $\mathrm{pH}$ homeostasis. Proceedings of the National Academy of Sciences, 101(39), 14073-14078. https://doi.org/10.1073/pnas.0405375101

Li, X.-Z., \& Nikaido, H. (2009). Efflux-Mediated Drug Resistance in Bacteria. Drugs, 69(12), 1555-1623. https://doi.org/10.2165/11317030-000000000-00000

Llano-Sotelo, B., Hickerson, R. P., Lancaster, L., Noller, H. F., \& Mankin, A. S. (2009). Fluorescently labeled ribosomes as a tool for analyzing antibiotic binding. $R N A, 15(8)$, 1597-1604. https://doi.org/10.1261/rna.1681609 
Lomovskaya, O., \& Lewis, K. (1992). Emr, an Escherichia coli locus for multidrug resistance. Proceedings of the National Academy of Sciences, 89(19), 8938-8942. https://doi.org/10.1073/pnas.89.19.8938

Lomovskaya, O, Lewis, K., \& Matin, A. (1995). EmrR is a negative regulator of the Escherichia coli multidrug resistance pump EmrAB. Journal of Bacteriology, 177(9), 2328-2334. https://doi.org/10.1128/JB.177.9.2328-2334.1995

Lomovskaya, Olga, Warren, M. S., Lee, A., Galazzo, J., Fronko, R., Lee, M., Blais, J., Cho, D., Chamberland, S., Renau, T., Leger, R., Hecker, S., Watkins, W., Hoshino, K., Ishida, H., \& Lee, V. J. (2001). Identification and Characterization of Inhibitors of Multidrug Resistance Efflux Pumps in Pseudomonas aeruginosa: Novel Agents for Combination Therapy. Antimicrobial Agents and Chemotherapy, 45(1), 105-116. https://doi.org/10.1128/AAC.45.1.105-116.2001

Long, F., Su, C.-C., Zimmermann, M. T., Boyken, S. E., Rajashankar, K. R., Jernigan, R. L., \& Yu, E. W. (2010). Crystal structures of the CusA efflux pump suggest methioninemediated metal transport. Nature, 467(7314), 484-488. https://doi.org/10.1038/nature09395

Lu, M., Radchenko, M., Symersky, J., Nie, R., \& Guo, Y. (2013). Structural insights into H+coupled multidrug extrusion by a MATE transporter. Nature Structural \& Molecular Biology, 20(11), 1310-1317. https://doi.org/10.1038/nsmb.2687

Malone, R. W., Felgner, P. L., \& Verma, I. M. (1989). Cationic liposome-mediated RNA transfection. Proceedings of the National Academy of Sciences, 86(16), 6077-6081. https://doi.org/10.1073/pnas.86.16.6077

Mandal, A. K., \& Argüello, J. M. (2003). Functional Roles of Metal Binding Domains of the Archaeoglobus fulgidus $\mathrm{Cu}+$-ATPase CopA. Biochemistry, 42(37), 11040-11047. https://doi.org/10.1021/bi034806y

Matias, V. R. F., Al-Amoudi, A., Dubochet, J., \& Beveridge, T. J. (2003). Cryo-Transmission Electron Microscopy of Frozen-Hydrated Sections of Escherichia coli and Pseudomonas aeruginosa. Journal of Bacteriology, 185(20), 6112-6118. https://doi.org/10.1128/JB.185.20.6112-6118.2003

Michael Green, N. (1990). [5] Avidin and streptavidin (pp. 51-67). https://doi.org/10.1016/0076-6879(90)84259-J

Miroux, B., \& Walker, J. E. (1996). Over-production of Proteins inEscherichia coli: Mutant Hosts that Allow Synthesis of some Membrane Proteins and Globular Proteins at High Levels. Journal of Molecular Biology, 260(3), 289-298. https://doi.org/10.1006/jmbi.1996.0399

Moore, B. (1960). A NEW SCREEN TEST AND SELECTIVE MEDIUM FOR THE RAPID DETECTION OF EPIDEMIC STRAINS OF STAPH. AUREUS. The Lancet, 276(7148), 453-458. https://doi.org/10.1016/S0140-6736(60)91591-9

Morita, Y., Kodama, K., Shiota, S., Mine, T., Kataoka, A., Mizushima, T., \& Tsuchiya, T. (1998). NorM, a putative multidrug efflux protein, of Vibrio parahaemolyticus and its homolog in Escherichia coli. Antimicrobial Agents and Chemotherapy, 42(7), 17781782. https://doi.org/10.1128/AAC.42.7.1778

Morrison, E. A., DeKoster, G. T., Dutta, S., Vafabakhsh, R., Clarkson, M. W., Bahl, A., 
Kern, D., Ha, T., \& Henzler-Wildman, K. A. (2012). Antiparallel EmrE exports drugs by exchanging between asymmetric structures. Nature, 481(7379), 45-50. https://doi.org/10.1038/nature10703

Moseng, M. A., Lyu, M., Pipatpolkai, T., Glaza, P., Emerson, C. C., Stewart, P. L., Stansfeld, P. J., \& Yu, E. W. (2021). Cryo-EM Structures of CusA Reveal a Mechanism of MetalIon Export. MBio, 12(2). https://doi.org/10.1128/mBio.00452-21

Müller, Reinke T. (2019). Substrate promiscuity of the AcrAB-TolC multidrug efflux pumpInsights from rationalized mutagenesis and NGS-assisted directed evolution screening (doctoral dissertation). Johann Wolfgang Goethe Universität, Frankfurt Am Main, Germany.

Müller, Reinke Tobias, \& Pos, K. M. (2015). The assembly and disassembly of the AcrABTolC three-component multidrug efflux pump. Biological Chemistry, 396(9-10), 10831089. https://doi.org/10.1515/hsz-2015-0150

Murakami, S., Nakashima, R., Yamashita, E., Matsumoto, T., \& Yamaguchi, A. (2006). Crystal structures of a multidrug transporter reveal a functionally rotating mechanism. Nature, 443(7108), 173-179. https://doi.org/10.1038/nature05076

Murakami, S., Nakashima, R., Yamashita, E., \& Yamaguchi, A. (2002). Crystal structure of bacterial multidrug efflux transporter AcrB. Nature, 419(6907), 587-593. https://doi.org/10.1038/nature01050

Nakashima, R., Sakurai, K., Yamasaki, S., Hayashi, K., Nagata, C., Hoshino, K., Onodera, Y., Nishino, K., \& Yamaguchi, A. (2013). Structural basis for the inhibition of bacterial multidrug exporters. Nature, 500(7460), 102-106. https://doi.org/10.1038/nature12300

Nanninga, N. (1998). Nanninga N. 1998. Microbiology and Molecular Biology Reviews.

Nemeth, J., Oesch, G., \& Kuster, S. P. (2015). Bacteriostatic versus bactericidal antibiotics for patients with serious bacterial infections: systematic review and meta-analysis. Journal of Antimicrobial Chemotherapy, 70(2), 382-395. https://doi.org/10.1093/jac/dku379

Nesterenko, M. V., Tilley, M., \& Upton, S. J. (1994). A simple modification of Blum's silver stain method allows for 30 minite detection of proteins in polyacrylamide gels. Journal of Biochemical and Biophysical Methods, 28(3), 239-242. https://doi.org/10.1016/0165022X(94)90020-5

Nies, D. H. (1999). Microbial heavy-metal resistance. Applied Microbiology and Biotechnology, 51(6), 730-750. https://doi.org/10.1007/s002530051457

Nikaido, H. (2003). Molecular Basis of Bacterial Outer Membrane Permeability Revisited. Microbiology and Molecular Biology Reviews, 67(4), 593-656. https://doi.org/10.1128/MMBR.67.4.593-656.2003

Nikaido, H. (2009). Multidrug Resistance in Bacteria. Annual Review of Biochemistry, 78(1), 119-146. https://doi.org/10.1146/annurev.biochem.78.082907.145923

Nikaido, H. (2011). Structure and mechanism of RND-type multidrug efflux pumps. Advances in Enzymology and Related Areas of Molecular Biology, 77, 1-60. https://doi.org/10.1002/9780470920541.ch1

Nikaido, H. (2018). RND transporters in the living world. Research in Microbiology, 169(7- 
8), 363-371. https://doi.org/10.1016/j.resmic.2018.03.001

Norrander, J., Kempe, T., \& Messing, J. (1983). Construction of improved M13 vectors using oligodeoxynucleotide-directed mutagenesis. Gene, 26(1), 101-106. https://doi.org/10.1016/0378-1119(83)90040-9

Ntsogo Enguéné, V. Y., VerchÃ“re, A., Phan, G., Broutin, I., \& Picard, M. (2015). Catch me if you can: a biotinylated proteoliposome affinity assay for the investigation of assembly of the MexA-MexB-OprM efflux pump from Pseudomonas aeruginosa. Frontiers in Microbiology, 6. https://doi.org/10.3389/fmicb.2015.00541

Ochman, H., Gerber, A. S., \& Hartl, D. L. (1988). Genetic applications of an inverse polymerase chain reaction. Genetics, 120(3), 621-623.

Odermatt, A., Suter, H., Krapf, R., \& Solioz, M. (1993). Primary structure of two P-type ATPases involved in copper homeostasis in Enterococcus hirae. The Journal of Biological Chemistry, 268(17), 12775-12779.

Oliero, M., Calvé, A., Fragoso, G., Cuisiniere, T., Hajjar, R., Dobrindt, U., \& Santos, M. M. (2021). Oligosaccharides increase the genotoxic effect of colibactin produced by pks+ Escherichia coli strains. BMC Cancer, 21(1). https://doi.org/10.1186/s12885-021-078768

Onyeji, C. O., Nightingale, C. H., \& Marangos, M. N. (1994). Enhanced killing of methicillin-resistantStaphylococcus aureus in human macrophages by liposomeentrapped vancomycin and teicoplanin. Infection, 22(5), 338-342. https://doi.org/10.1007/BF01715542

Opperman, T. J., Kwasny, S. M., Kim, H.-S., Nguyen, S. T., Houseweart, C., D’Souza, S., Walker, G. C., Peet, N. P., Nikaido, H., \& Bowlin, T. L. (2014). Characterization of a Novel Pyranopyridine Inhibitor of the AcrAB Efflux Pump of Escherichia coli. Antimicrobial Agents and Chemotherapy, 58(2), 722-733. https://doi.org/10.1128/AAC.01866-13

Opperman, T. J., \& Nguyen, S. T. (2015). Recent advances toward a molecular mechanism of efflux pump inhibition. Frontiers in Microbiology, 6. https://doi.org/10.3389/fmicb.2015.00421

Origin 8.6. OriginLab Corporation, Northampton, MA, USA.

Pal, C., Asiani, K., Arya, S., Rensing, C., Stekel, D. J., Larsson, D. G. J., \& Hobman, J. L. (2017). Metal Resistance and Its Association With Antibiotic Resistance (pp. 261-313). https://doi.org/10.1016/bs.ampbs.2017.02.001

Pankey, G. A., \& Sabath, L. D. (2004). Clinical Relevance of Bacteriostatic versus Bactericidal Mechanisms of Action in the Treatment of Gram-Positive Bacterial Infections. Clinical Infectious Diseases, 38(6), 864-870. https://doi.org/10.1086/381972

Pasqua, Grossi, Zennaro, Fanelli, Micheli, Barras, Colonna, \& Prosseda. (2019). The Varied Role of Efflux Pumps of the MFS Family in the Interplay of Bacteria with Animal and Plant Cells. Microorganisms, 7(9), 285. https://doi.org/10.3390/microorganisms7090285

Paulowski, L., Donoghue, A., Nehls, C., Groth, S., Koistinen, M., Hagge, S. O., Böhling, A., Winterhalter, M., \& Gutsmann, T. (2020). The Beauty of Asymmetric Membranes: Reconstitution of the Outer Membrane of Gram-Negative Bacteria. Frontiers in Cell and 
Developmental Biology, 8. https://doi.org/10.3389/fcell.2020.00586

Philippon, A., Arlet, G., \& Jacoby, G. A. (2002). Plasmid-Determined AmpC-Type $\beta$ Lactamases. Antimicrobial Agents and Chemotherapy, 46(1), 1-11. https://doi.org/10.1128/AAC.46.1.1-11.2002

Picard, M., Verchère, A., \& Broutin, I. (2012). Monitoring the active transport of efflux pumps after their reconstitution into proteoliposomes: Caveats and keys. Analytical Biochemistry, 420(2), 194-196. https://doi.org/10.1016/j.ab.2011.09.025

Poirel, L., Madec, J.-Y., Lupo, A., Schink, A.-K., Kieffer, N., Nordmann, P., \& Schwarz, S. (2018). Antimicrobial Resistance in Escherichia coli. In Antimicrobial Resistance in Bacteria from Livestock and Companion Animals. American Society of Microbiology. https://doi.org/10.1128/microbiolspec.ARBA-0026-2017

Poolman, B., Royer, T. J., Mainzer, S. E., \& Schmidt, B. F. (1989). Lactose transport system of Streptococcus thermophilus: a hybrid protein with homology to the melibiose carrier and enzyme III of phosphoenolpyruvate-dependent phosphotransferase systems. Journal of Bacteriology, 171(1), 244-253. https://doi.org/10.1128/JB.171.1.244-253.1989

Poolman, J. T., \& Wacker, M. (2016). Extraintestinal Pathogenic Escherichia coli, a Common Human Pathogen: Challenges for Vaccine Development and Progress in the Field. Journal of Infectious Diseases, 213(1). https://doi.org/10.1093/infdis/jiv429

Pos, K. M. (2009). Drug transport mechanism of the AcrB efflux pump. Biochimica et Biophysica Acta (BBA) - Proteins and Proteomics, 1794(5), 782-793. https://doi.org/10.1016/j.bbapap.2008.12.015

Pos, K. M., \& Diederichs, K. (2002). Purification, crystallization and preliminary diffraction studies of AcrB, an inner-membrane multi-drug efflux protein. Acta Crystallographica Section D Biological Crystallography, 58(10), 1865-1867. https://doi.org/10.1107/S0907444902013963

Qiu, W., Fu, Z., Xu, G. G., Grassucci, R. A., Zhang, Y., Frank, J., Hendrickson, W. A., \& Guo, Y. (2018). Structure and activity of lipid bilayer within a membrane-protein transporter. Proceedings of the National Academy of Sciences, 115(51), 12985-12990. https://doi.org/10.1073/pnas.1812526115

Rabilloud, T. (1990). Mechanisms of protein silver staining in polyacrylamide gels: A 10$\begin{array}{llll}\text { year } & \text { synthesis. } & \text { Electrophoresis, }\end{array}$ https://doi.org/10.1002/elps.1150111003

Radestock, S., \& Forrest, L. R. (2011). The Alternating-Access Mechanism of MFS Transporters Arises from Inverted-Topology Repeats. Journal of Molecular Biology, 407(5), 698-715. https://doi.org/10.1016/j.jmb.2011.02.008

Raetz, C. R. H. (1986). Molecular Genetics of Membrane Phospholipid Synthesis. Annual Review of Genetics, 20(1), 253-291. https://doi.org/10.1146/annurev.ge.20.120186.001345

Rastegar Lari, A., Gold, F., Borderon, J. C., Laugier, J., \& Lafont, J.-P. (1990). Implantation and in vivo Antagonistic Effects of Antibiotic-Susceptible \&lt;i\&gt;Escherichia Coli\&lt;/i\&gt; Strains Administered to Premature Newborns. Neonatology, 58(2). https://doi.org/10.1159/000243235

Rawat, D., \& Nair, D. (2010). Extended-spectrum $\beta$-lactamases in Gram Negative Bacteria. 
Journal of Global Infectious Diseases, 2(3), 263-274. https://doi.org/10.4103/0974777X.68531

Rensing, C., Fan, B., Sharma, R., Mitra, B., \& Rosen, B. P. (2000). CopA: An Escherichia coli $\mathrm{Cu}(\mathrm{I})$-translocating P-type ATPase. Proceedings of the National Academy of Sciences, 97(2), 652-656. https://doi.org/10.1073/pnas.97.2.652

Rigaud, J.-L., \& Lévy, D. (2003). Reconstitution of Membrane Proteins into Liposomes (pp. 65-86). https://doi.org/10.1016/S0076-6879(03)72004-7

Ruggerone, P., Murakami, S., M. Pos, K., \& Vargiu, A. (2013). RND Efflux Pumps: Structural Information Translated into Function and Inhibition Mechanisms. Current Topics in Medicinal Chemistry, 13(24), 3079-3100. https://doi.org/10.2174/15680266113136660220

Schamberger, G. P., Phillips, R. L., Jacobs, J. L., \& Diez-Gonzalez, F. (2004). Reduction of Escherichia coli O157:H7 Populations in Cattle by Addition of Colicin E7-Producing E. coli to Feed. Applied and Environmental Microbiology, 70(10). https://doi.org/10.1128/AEM.70.10.6053-6060.2004

Schiffelers, R. (2001). Liposome-encapsulated aminoglycosides in pre-clinical and clinical studies. Journal of Antimicrobial Chemotherapy, 48(3), 333-344. https://doi.org/10.1093/jac/48.3.333

Schmidt, T. H., Raunest, M., Fischer, N., Reith, D., \& Kandt, C. (2016). Computer simulations suggest direct and stable tip to tip interaction between the outer membrane channel TolC and the isolated docking domain of the multidrug RND efflux transporter AcrB. Biochimica et Biophysica Acta (BBA) - Biomembranes, 1858(7), 1419-1426. https://doi.org/10.1016/j.bbamem.2016.03.029

Schneider, C. A., Rasband, W. S., \& Eliceiri, K. W. (2012). NIH Image to ImageJ: 25 years of image analysis. Nature Methods, 9(7), 671-675. https://doi.org/10.1038/nmeth.2089

Schwarz, S., Kehrenberg, C., Doublet, B., \& Cloeckaert, A. (2004). Molecular basis of bacterial resistance to chloramphenicol and florfenicol. FEMS Microbiology Reviews, 28(5), 519-542. https://doi.org/10.1016/j.femsre.2004.04.001

Seeger, M. A. (2006). Structural Asymmetry of AcrB Trimer Suggests a Peristaltic Pump Mechanism. Science, 313(5791), 1295-1298. https://doi.org/10.1126/science.1131542

Seeger, Markus A., von Ballmoos, C., Verrey, F., \& Pos, K. M. (2009). Crucial Role of Asp408 in the Proton Translocation Pathway of Multidrug Transporter AcrB: Evidence from Site-Directed Mutagenesis and Carbodiimide Labeling. Biochemistry, 48(25), 5801-5812. https://doi.org/10.1021/bi900446j

Sengupta, S., Chattopadhyay, M. K., \& Grossart, H.-P. (2013). The multifaceted roles of antibiotics and antibiotic resistance in nature. Frontiers in Microbiology, 4. https://doi.org/10.3389/fmicb.2013.00047

Sennhauser, G., Amstutz, P., Briand, C., Storchenegger, O., \& Grütter, M. G. (2006). Drug Export Pathway of Multidrug Exporter AcrB Revealed by DARPin Inhibitors. PLoS Biology, 5(1), e7. https://doi.org/10.1371/journal.pbio.0050007

Sennhauser, G., Bukowska, M. A., Briand, C., \& Grütter, M. G. (2009). Crystal Structure of the Multidrug Exporter MexB from Pseudomonas aeruginosa. Journal of Molecular Biology, 389(1), 134-145. https://doi.org/10.1016/j.jmb.2009.04.001 
Shen, L. L. (1993). Quinolone Antibacterial Agents. Washington, Vol. 2.

Shi, X., Chen, M., Yu, Z., Bell, J. M., Wang, H., Forrester, I., Villarreal, H., Jakana, J., Du, D., Luisi, B. F., Ludtke, S. J., \& Wang, Z. (2019). In situ structure and assembly of the multidrug efflux pump AcrAB-TolC. Nature Communications, 10(1), 2635. https://doi.org/10.1038/s41467-019-10512-6

Silver, S. (2003). Bacterial silver resistance: molecular biology and uses and misuses of silver compounds. FEMS Microbiology Reviews, 27(2-3), 341-353. https://doi.org/10.1016/S0168-6445(03)00047-0

Sjuts, H., Vargiu, A. V., Kwasny, S. M., Nguyen, S. T., Kim, H.-S., Ding, X., Ornik, A. R., Ruggerone, P., Bowlin, T. L., Nikaido, H., Pos, K. M., \& Opperman, T. J. (2016). Molecular basis for inhibition of AcrB multidrug efflux pump by novel and powerful pyranopyridine derivatives. Proceedings of the National Academy of Sciences, 113(13), 3509-3514. https://doi.org/10.1073/pnas.1602472113

Smith, K. E., Wilker, P. R., Reiter, P. L., Hedican, E. B., Bender, J. B., \& Hedberg, C. W. (2012). Antibiotic Treatment of Escherichia coli O157 Infection and the Risk of Hemolytic Uremic Syndrome, Minnesota. Pediatric Infectious Disease Journal, 31(1). https://doi.org/10.1097/INF.0b013e31823096a8

Songsungthong, W., Prasopporn, S., Bohan, L., Srimanote, P., Leartsakulpanich, U., \& Yongkiettrakul, S. (2021). A novel bicyclic 2,4-diaminopyrimidine inhibitor of Streptococcus suis dihydrofolate reductase. PeerJ, 9, e10743. https://doi.org/10.7717/peerj.10743

Stoyanov, J. V, Magnani, D., \& Solioz, M. (2003). Measurement of cytoplasmic copper, silver, and gold with a lux biosensor shows copper and silver, but not gold, efflux by the CopA ATPase of Escherichia coli. FEBS Letters, 546(2-3), 391-394. https://doi.org/10.1016/S0014-5793(03)00640-9

Straus, S. K., \& Hancock, R. E. W. (2006). Mode of action of the new antibiotic for Grampositive pathogens daptomycin: Comparison with cationic antimicrobial peptides and lipopeptides. Biochimica et Biophysica Acta (BBA) - Biomembranes, 1758(9), 12151223. https://doi.org/10.1016/j.bbamem.2006.02.009

Su, C.-C., Long, F., Zimmermann, M. T., Rajashankar, K. R., Jernigan, R. L., \& Yu, E. W. (2011). Crystal structure of the CusBA heavy-metal efflux complex of Escherichia coli. Nature, 470(7335), 558-562. https://doi.org/10.1038/nature09743

Sugawara, E., Nestorovich, E. M., Bezrukov, S. M., \& Nikaido, H. (2006). Pseudomonas aeruginosa Porin OprF Exists in Two Different Conformations. Journal of Biological Chemistry, 281(24), 16220-16229. https://doi.org/10.1074/jbc.M600680200

Sulavik, M. C., Houseweart, C., Cramer, C., Jiwani, N., Murgolo, N., Greene, J., DiDomenico, B., Shaw, K. J., Miller, G. H., Hare, R., \& Shimer, G. (2001). Antibiotic Susceptibility Profiles ofEscherichia coli Strains Lacking Multidrug Efflux Pump Genes. Antimicrobial Agents and Chemotherapy, 45(4), 1126-1136. https://doi.org/10.1128/AAC.45.4.1126-1136.2001

Tal, N., \& Schuldiner, S. (2009). A coordinated network of transporters with overlapping specificities provides a robust survival strategy. Proceedings of the National Academy of Sciences, 106(22), 9051-9056. https://doi.org/10.1073/pnas.0902400106 
Tamura, N., Murakami, S., Oyama, Y., Ishiguro, M., \& Yamaguchi, A. (2005). Direct Interaction of Multidrug Efflux Transporter AcrB and Outer Membrane Channel TolC Detected via Site-Directed Disulfide Cross-Linking $\dagger$. Biochemistry, 44(33), 1111511121. https://doi.org/10.1021/bi050452u

Tikhonova, E. B., \& Zgurskaya, H. I. (2004). AcrA, AcrB, and TolC of Escherichia coli Form a Stable Intermembrane Multidrug Efflux Complex. Journal of Biological Chemistry, 279(31), 32116-32124. https://doi.org/10.1074/jbc.M402230200

Touzé, T., Eswaran, J., Bokma, E., Koronakis, E., Hughes, C., \& Koronakis, V. (2004). Interactions underlying assembly of the Escherichia coli AcrAB-TolC multidrug efflux system. Molecular Microbiology, 53(2), 697-706. https://doi.org/10.1111/j.13652958.2004.04158.x

Towbin, H., Staehelin, T., \& Gordon, J. (1979). Electrophoretic transfer of proteins from polyacrylamide gels to nitrocellulose sheets: procedure and some applications. Proceedings of the National Academy of Sciences, 76(9), 4350-4354. https://doi.org/10.1073/pnas.76.9.4350

Trimble, M. J., Mlynárčik, P., Kolář, M., \& Hancock, R. E. W. (2016). Polymyxin: Alternative Mechanisms of Action and Resistance. Cold Spring Harbor Perspectives in Medicine, 6(10). https://doi.org/10.1101/cshperspect.a025288

Tsutsumi, K., Yonehara, R., Ishizaka-Ikeda, E., Miyazaki, N., Maeda, S., Iwasaki, K., Nakagawa, A., \& Yamashita, E. (2019). Structures of the wild-type MexAB-OprM tripartite pump reveal its complex formation and drug efflux mechanism. Nature Communications, 10(1), 1520. https://doi.org/10.1038/s41467-019-09463-9

The PyMOL Molecular Graphics System, Version 2.2.0 Schrödinger, LLC.

Ullah, H., \& Ali, S. (2017). Classification of Anti-Bacterial Agents and Their Functions. In Antibacterial Agents. InTech. https://doi.org/10.5772/intechopen.68695

van Duijkeren, E., Schink, A.-K., Roberts, M. C., Wang, Y., \& Schwarz, S. (2018). Mechanisms of Bacterial Resistance to Antimicrobial Agents. In Antimicrobial Resistance in Bacteria from Livestock and Companion Animals (pp. 51-82). ASM Press. https://doi.org/10.1128/9781555819804.ch4

Vargiu, A. V., Ruggerone, P., Opperman, T. J., Nguyen, S. T., \& Nikaido, H. (2014). Molecular Mechanism of MBX2319 Inhibition of Escherichia coli AcrB Multidrug Efflux Pump and Comparison with Other Inhibitors. Antimicrobial Agents and Chemotherapy, 58(10), 6224-6234. https://doi.org/10.1128/AAC.03283-14

Ventola, C. L. (2015). The antibiotic resistance crisis: part 1: causes and threats. $P \& T: A$ Peer-Reviewed Journal for Formulary Management, 40(4), 277-283.

Verchère, A. (2014). Functional investigation of the efflux pump MexA-MexB-OprM of Pseudomonas aeruginosa (doctoral dissertation). Biomolecules [q-Bio.BM]. Université René Descartes - Paris V, English. NNT : 2014PA05P622ff. fftel-01160389.

Verchère, A., Broutin, I., \& Picard, M. (2012). Photo-induced proton gradients for the in vitro investigation of bacterial efflux pumps. Scientific Reports, 2(1), 306. https://doi.org/10.1038/srep00306

Verchère, A., Dezi, M., Adrien, V., Broutin, I., \& Picard, M. (2015). In vitro transport 
activity of the fully assembled MexAB-OprM efflux pump from Pseudomonas aeruginosa. Nature Communications, 6(1), 6890. https://doi.org/10.1038/ncomms7890

Vollaard, E. J., \& Clasener, H. A. (1994). Colonization resistance. Antimicrobial Agents and Chemotherapy, 38(3). https://doi.org/10.1128/AAC.38.3.409

Wagner, S., Klepsch, M. M., Schlegel, S., Appel, A., Draheim, R., Tarry, M., Hogbom, M., van Wijk, K. J., Slotboom, D. J., Persson, J. O., \& de Gier, J.-W. (2008). Tuning Escherichia coli for membrane protein overexpression. Proceedings of the National Academy of Sciences, 105(38), 14371-14376. https://doi.org/10.1073/pnas.0804090105

Wall, S. (2019). Prevention of antibiotic resistance - an epidemiological scoping review to identify research categories and knowledge gaps. Global Health Action, 12(sup1), 1756191. https://doi.org/10.1080/16549716.2020.1756191

Wallin, E., \& von Heijne, G. (1998). Genome-wide analysis of integral membrane proteins from eubacterial, archaean, and eukaryotic organisms. Protein Science: A Publication of the Protein Society, 7(4), 1029-1038. https://doi.org/10.1002/pro.5560070420

Walsh, C. (2000). Molecular mechanisms that confer antibacterial drug resistance. Nature, 406(6797), 775-781. https://doi.org/10.1038/35021219

Walzer, P. D., Kim, C. K., Foy, J. M., Linke, M. J., \& Cushion, M. T. (1988). Inhibitors of folic acid synthesis in the treatment of experimental Pneumocystis carinii pneumonia. Antimicrobial Agents and Chemotherapy, 32(1), 96-103. https://doi.org/10.1128/AAC.32.1.96

Wang, Z., Fan, G., Hryc, C. F., Blaza, J. N., Serysheva, I. I., Schmid, M. F., Chiu, W., Luisi, B. F., \& Du, D. (2017). An allosteric transport mechanism for the AcrAB-TolC multidrug efflux pump. ELife, 6. https://doi.org/10.7554/eLife.24905

Wardlaw, T., Salama, P., Brocklehurst, C., Chopra, M., \& Mason, E. (2010). Diarrhoea: why children are still dying and what can be done. The Lancet, 375(9718), 870-872. https://doi.org/10.1016/S0140-6736(09)61798-0

Warren, G. B., Toon, P. A., Birdsall, N. J., Lee, A. G., \& Metcalfe, J. C. (1974). Reconstitution of a calcium pump using defined membrane components. Proceedings of the National Academy of Sciences of the United States of America, 71(3), 622-626. https://doi.org/10.1073/pnas.71.3.622

Watson, H. (2015). Biological membranes. Essays in Biochemistry, 59, 43-69. https://doi.org/10.1042/bse0590043

Weiner, N., Martin, F., \& Riaz, M. (1989). Liposomes as a Drug Delivery System. Drug Development and Industrial Pharmacy, 15(10), 1523-1554. https://doi.org/10.3109/03639048909052502

Welch, A., Awah, C. U., Jing, S., van Veen, H. W., \& Venter, H. (2010). Promiscuous partnering and independent activity of MexB, the multidrug transporter protein from Pseudomonas aeruginosa. Biochemical Journal, 430(2), 355-364. https://doi.org/10.1042/BJ20091860

Wijekoon, C. J. K., Udagedara, S. R., Knorr, R. L., Dimova, R., Wedd, A. G., \& Xiao, Z. (2017). Copper ATPase CopA from Escherichia coli : Quantitative Correlation between ATPase Activity and Vectorial Copper Transport. Journal of the American Chemical Society, 139(12), 4266-4269. https://doi.org/10.1021/jacs.6b12921 
Wilkins, M. R., Gasteiger, E., Bairoch, A., Sanchez, J.-C., Williams, K. L., Appel, R. D., \& Hochstrasser, D. F. (1999). Protein Identification and Analysis Tools in the ExPASy Server. In 2-D Proteome Analysis Protocols (pp. 531-552). Humana Press. https://doi.org/10.1385/1-59259-584-7:531

Wilson, D. N. (2014). Ribosome-targeting antibiotics and mechanisms of bacterial resistance. Nature Reviews Microbiology, 12(1), 35-48. https://doi.org/10.1038/nrmicro3155

Yin, Y. (2006). Structure of the Multidrug Transporter EmrD from Escherichia coli. Science, 312(5774), 741-744. https://doi.org/10.1126/science.1125629

Yousefian, N., Ornik-Cha, A., Poussard, S., Decossas, M., Berbon, M., Daury, L., Taveau, J.C., Dupuy, J.-W., Đorđević-Marquardt, S., Lambert, O., \& Pos, K. M. (2021). Structural characterization of the EmrAB-TolC efflux complex from E. coli. Biochimica et $\begin{array}{lllll}\text { Biophysica Acta (BBA) - Biomembranes, } & 183488 .\end{array}$ https://doi.org/10.1016/j.bbamem.2020.183488

Yue, L., Jiang, H.-X., Liao, X.-P., Liu, J.-H., Li, S.-J., Chen, X.-Y., Chen, C.-X., Lü, D.-H., \& Liu, Y.-H. (2008). Prevalence of plasmid-mediated quinolone resistance qnr genes in poultry and swine clinical isolates of Escherichia coli. Veterinary Microbiology, 132(34), 414-420. https://doi.org/10.1016/j.vetmic.2008.05.009

Zampieri, M., Enke, T., Chubukov, V., Ricci, V., Piddock, L., \& Sauer, U. (2017). Metabolic constraints on the evolution of antibiotic resistance. Molecular Systems Biology, 13(3), 917. https://doi.org/10.15252/msb.20167028

Zgurskaya, H. I., \& Nikaido, H. (1999). Bypassing the periplasm: Reconstitution of the AcrAB multidrug efflux pump of Escherichia coli. Proceedings of the National Academy of Sciences, 96(13), 7190-7195. https://doi.org/10.1073/pnas.96.13.7190

Zgurskaya, Helen I., Krishnamoorthy, G., Ntreh, A., \& Lu, S. (2011). Mechanism and Function of the Outer Membrane Channel TolC in Multidrug Resistance and Physiology of Enterobacteria. Frontiers in Microbiology, 2. https://doi.org/10.3389/fmicb.2011.00189 


\section{Abbreviations}

A

$\mathrm{ABC}$

ACMA

AcrA

AcrB

AG

AP

APS

ATP

BCIP

BN-PAGE

Carb

$\mathrm{CCCP}$

CopA

$\mathrm{Cm}$

CMC

CRC

CusA

DBP

DDM

DHFR

DHPS
Ampere

ATP-Binding Cassette

9-amino-6-chloro-2-methoxyacridine

Acriflavine resistance protein A

Acriflavine resistance protein $\mathrm{B}$

aminoglycoside

Access pocket

Ammonium persulfate

Adenosine triphosphate

5-Bromo-4-chloro-3- indolyl phosphate

Blue Native-PolyAcrylamide Gel Electrophoresis

Carbenicillin

Carbonyl cyanide m-chlorophenylhydrazone

Copper-exporting P-type ATPase A

Chloramphenicol

Critical micelle concentration

Colitis-associated colorectal cancer

Cation efflux system protein CusA

Deep binding pocket

n-Dodecyl $\beta$-D-maltoside

Dihydrofolate reductase

Dihydropteroate synthase 
DNA Deoxyribonucleic acid

DNase I Deoxyribonuclease I

dNTP 2'-Deoxy-nucleoside-5'-triphosphate

E. coli Escherichia coli

EM Electron microscopy

EmrA Multidrug export protein EmrA

EmrB Multidrug export protein EmrB

ETEC Enterotoxigenic E. coli

FC (DC) C-terminal Funnel (Docking) Domain

FD Funnel Domain

Ff Florfenicol

FN (DN) N-terminal Funnel (Docking) Domain

FX Fragment exchange

GFP Green Fluorescent Protein

$\mathrm{H} \quad$ Hours

His-tag Histidine-tag

HEPES 2-(4-(2-hydroxyethyl)-1-piperazineethanesulfonic acid

HUS Bloody diarrhoea uremic syndrome

IMAC Ion metal affinity chromatography

IMP Inner membrane protein

IPTG Isopropyl $\beta$-D-1-thiogalactopyranoside

ITC Isothermal Titration Calorimetry

$\mathrm{kDa} \quad$ Kilodalton

KAN Kanamycin 
$\mathrm{Km} \quad$ Substrate concentration at which the reaction rate is half of Vmax

L Loose (conformation)

LB Lysogeny Broth

LPS Lipopolysaccharides

$\mu \mathrm{S} \quad$ Microseconds

$\mathrm{mV} \quad$ Millivolt

MATE Multidrug and Toxic compounds Extrusion

MDR Multidrug resistance

MFP Membrane fusion protein

MFS Major Facilitator Superfamily

MH Mueller-Hinton

MIC Minimal inhibitory concentration

MPD Membrane proximal domain

MP-GFP Membrane protein-green fluorescent protein

MW Molecular Weight

NBD Nucleotide Binding Domain

Ni-NTA Nickel-ion-nitrilotriacetic acid

NBT Nitro-blue tetrazolium

NMR Nuclear Magnetic Resonance

O Open (conformation)

OD600 Optical density at $600 \mathrm{~nm}$

OG n-Octyl ß-D-glucopyranoside

OM Outer Membrane

OMF Outer membrane factor 
o/n Overnight

PACE Proteobacterial Antimicrobial Compound Efflux

PC1 C-terminal Porter-subdomain 1

PC2 C-terminal Porter-subdomain 2

PCR Polymerase Chain Reaction

PD Porter Domain

PDA Plate dilution assay

PDB Protein Data Bank

PGSK Phen Green SK

PL Phospholipid

PMFS Phenylmethylsulphonyl fluoride

Pyranine 8-hydroxy-1,3,6-pyrene-trisulfonate

RFP Red Fluorescent Protein

RND Resistance Nodulation cell Division

rpm Revolutions per minute

s $\quad$ Seconds

SBD Substrate Binding Domain

SDS Sodium dodecyl sulfate

SDS-PAA Sodium dodecyl sulfate-polyacrylamide

SDS-PAGE Sodium dodecyl sulfate-polyacrylamide gel electrophoresis

SEC Size exclusion chromatography

SMR Small Multidrug Resistance

$\mathrm{T} \quad$ Tight (conformation)

TB Terrific Broth 
TEMED Tetramethyl ethylenediamine

TolC Tolerance Colicin E1

Tris Tris(hydroxymethyl)aminomethane

TM Transmembrane helix

TMD Transmembrane domain

UV Ultraviolet

V Volt

$\mathrm{mV} \quad$ Millivolt

Vmax Maximum rate achieved by the system at saturating substrate concentration

WW2 World War 2 


\section{Lists of figures and tables}

Figure 1. Schematic representation of the structure of the cell envelope of $E$. coli............... 11

Figure 2. Schematic representations of the introduction of antibiotics on the market and

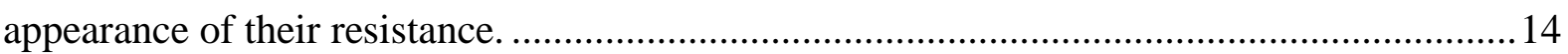

Figure 3. Schematic representation of drug targets in the bacterial cell ............................... 15

Figure 4. Mechanisms of antibiotic resistance in bacteria.................................................... 19

Figure 5. Multidrug efflux pumps in Gram-negative bacteria............................................23

Figure 6. Proposed mechanism of silver efflux in Gram-negative bacteria. ..........................28

Figure 7. The organization of AcrABZ-TolC complex from E. coli (PDB: 5O66)................29

Figure 8. Structural characteristics of efflux pump AcrB ................................................... 30

Figure 9. Suggested mechanism of proton transport through AcrB. .....................................33

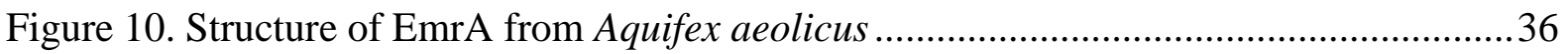

Figure 11. Proposed model of CusCBA efflux pump.......................................................... 38

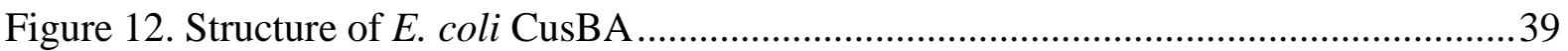

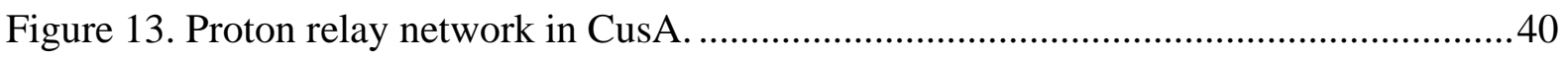

Figure 14. Schematic representation of the bilayer membrane of liposome.......................... 43

Figure 15. Schematic representation of unilamellar and multilamellar liposomes.................44

Figure 16. Schematic representation of the steps of the liposome preparation. ......................45

Figure 17. Steps of the detergent-mediated reconstitution... ................................................ 48

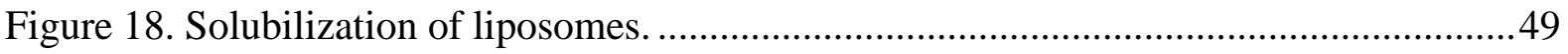

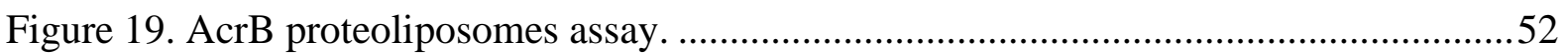

Figure 20. Schematic representation of the MexAB-OprM in vitro assay.............................53

Figure 21. Schematic representation of the pull-down experiment with MexAB and OprM

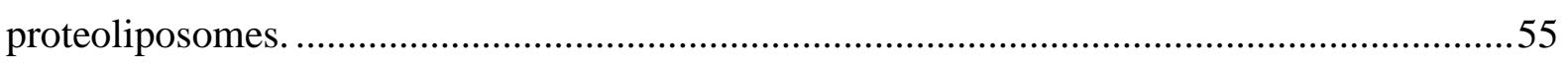

Figure 22. In vitro investigation of flux events mediated by AcrAB-TolC........................... 87

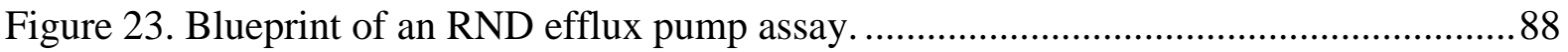

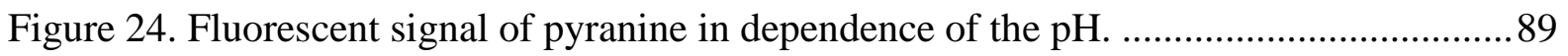

Figure 25. Characterization of the purity, monodispersity and oligomeric state of AcrA_A200V.

Figure 26. Characterization of the purity, monodispersity, and oligomeric state of AcrB wild type. 
Figure 27. Characterization of the purity, monodispersity and oligomeric state of AcrB_D407N.

Figure 28. Characterization of the purity, monodispersity and oligomeric state of TolC wild type. .94

Figure 29. $\mathrm{H}^{+}$-transport in AcrB proteoliposomes and non-protein containing liposomes. ....95

Figure 30. $\mathrm{H}^{+}$-transport in $\mathrm{Acr} A B$ proteoliposomes and non-protein containing liposomes. .96

Figure 31. $\mathrm{H}^{+}$-transport in AcrB and AcrAB proteoliposomes............................................97

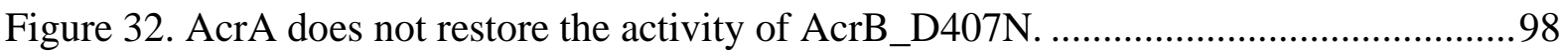

Figure 33. Structures of AcrB inhibitors, MBX3132 and Phe-Arg- $\beta$-naphthylamide (PA $\beta N$;

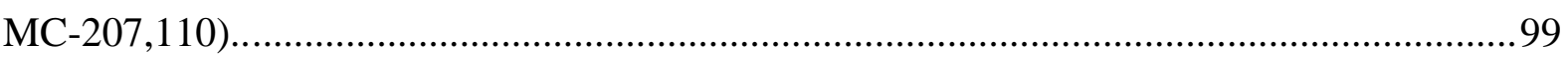

Figure 34. $\mathrm{H}^{+}$-transport in AcrB and AcrAB proteoliposomes...........................................99

Figure 35. General influence of MBX3132 on the liposomes and protein activity...............100

Figure 36. Effect of Phe-Arg- $\beta$-naphthylamide $(\mathrm{PA} \beta \mathrm{N})$ on $\mathrm{H}^{+}$influx into proteoliposomes

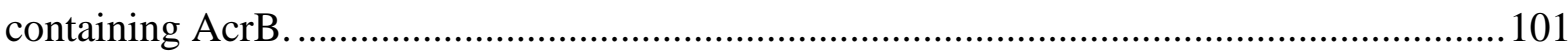

Figure 37. $\mathrm{H}^{+}$-transport in AcrB_F628L proteoliposomes. ................................................. 103

Figure 38. Streptavidin-biotin complex like a tool for proving AcrAB-TolC complex formation

Figure 39. The pull-down assay for the observations of the association and dissociation of the

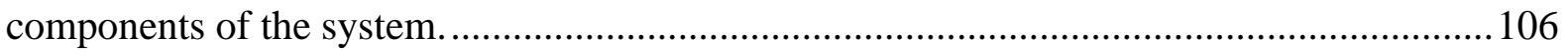

Figure 40. Pull-down experiments with AcrAB biotinylated proteoliposomes..................... 109

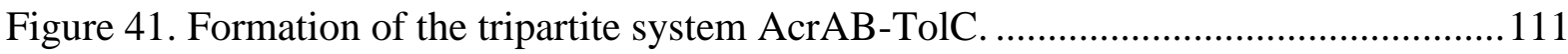

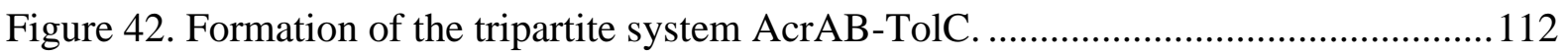

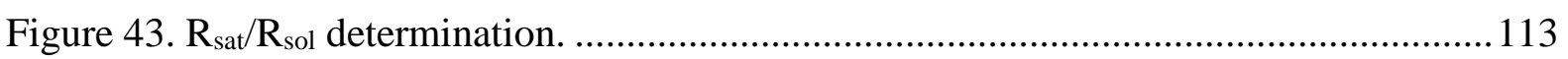

Figure 44. Solubilization assay of AcrA, AcrB, TolC and AcrAB proteoliposomes............ 115

Figure 45. TolC proteoliposome pyranine efflux assay................................................... 118

Figure 46. Complete solubilization of liposomes leads to loss of fluorescent signal............119

Figure 47. CopA, a P-type ATPase, expels silver and copper ions from the cytoplasm of

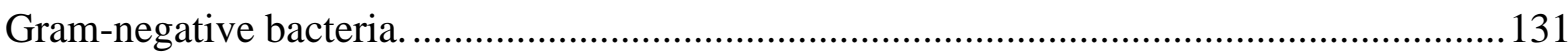

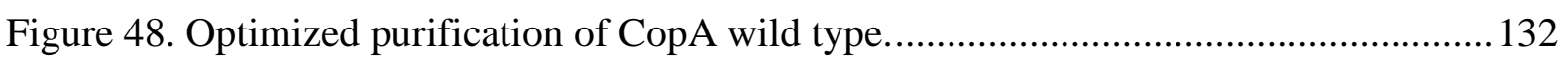

Figure 49. The ATP-NADH assay is based on a reaction in which ATP hydrolysis is coupled

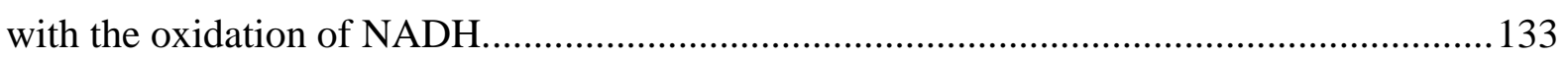

Figure 50. The kinetics of the ATPase activity of purified CopA in detergent micelles....... 134

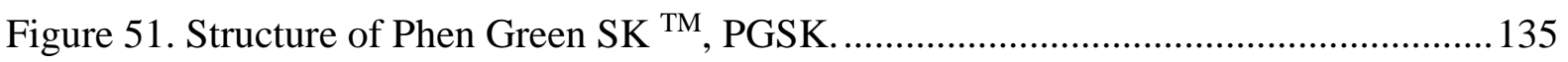

Figure 52. Phen Green SK dye was titrated with different concentrations of silver nitrate. .137 
Figure 54. Solubilization assay of CopA wild type proteoliposomes, prepared for usage with ACMA. 138

Figure 55. CopA reconstituted in liposomes shows activity. 139

Figure 56. CopA reconstituted in liposomes in the presence of sodium azide. 141

Figure 57. CopA reconstituted in liposomes does not show substantial quenching of PGSK in absence of silver ions. 141

Figure 58. Suggested pathway of silver ions through CusA efflux pump.... 146

Figure 59. Purification of CusA wild type. 147

Figure 60. Purification of CusA D405N 148

Figure 61. Solubilization assay of CusA wild type. 149

Figure 62. $\mathrm{Ag}^{+} / \mathrm{H}^{+}$antiport of the CusA efflux pump in the two expected different orientations of RND pump after reconstitution into liposomes. 150

Figure 63. CusA-mediated ACMA quenching. 152

Figure 64. CusA-mediated ACMA quenching. 153

Figure 65. CusA wild type shows activity when reconstituted into the liposomes. 153

Figure 66. CusA-mediated ACMA quenching. 154

Figure 67. CusA-mediated ACMA quenching. 156

Figure 68. CusA-mediated ACMA quenching. 157

Figure 69. CusA-mediated ACMA quenching. 158

Figure 70. CusA-mediated $\mathrm{Ag}^{+}$-transport in proteoliposomes. 159

Figure $71 . \mathrm{Ag}^{+}$-transport into CusA-containing proteoliposomes. 161

Figure 72. Mechanism of substrate-proton antiport in AcrB. 165

Figure 73. Possible transport pathways of protons and silver ions through CusA efflux pump 170

Figure 74. Substrate transport pathway and structural analysis of EmrAB-TolC efflux pump. 173

Figure 75. Agarose gel of the vectors harbouring pRSFDMG_emrA_tolC (7280 bp) and

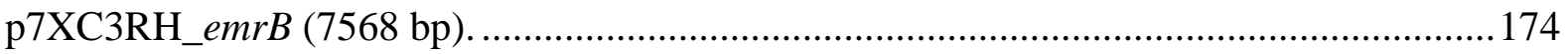

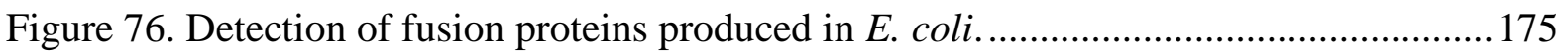

Figure 77. Drug susceptibility profile of E. coli BW25113(DE3) $\triangle a c r A B$ strain on solid medium.

Figure 78. Drug susceptibility test of E. coli BW25113(DE3 $\triangle a c r A B$ harbouring pRSFDMG_emrA_tolC and p7XC3RH_emrB. 
Figure 79. Drug susceptibility test of $E$. coli BW25113(DE3) $\triangle a c r A B$ harbouring p7XC3RH_emrB 178

Figure 80 . Drug susceptibility test of $E$. coli strains harbouring pRSFDMG_emrA_tolC and p7XC3RH_emrB.

Figure 81. Drug susceptibility test of $E$. coli C41(DE3) wild type and C41(DE3) $\triangle a c r A B$ harbouring pRSFDMG_emrA_tolC and p7XC3RH_emrB. 180

Figure 82. Drug susceptibility test of $E$. coli C41(DE3) wild type and C41(DE3) $\triangle a c r A B$ harbouring pRSFDMG_emrA_tolC and p7XC3RH_emrB.

Table 1. Laboratory equipment used in the thesis. .57

Table 2. Bacterial strains used in the thesis. .59

Table 3. Plasmids used in the thesis. .60

Table 4. Primers used for FX cloning. 60

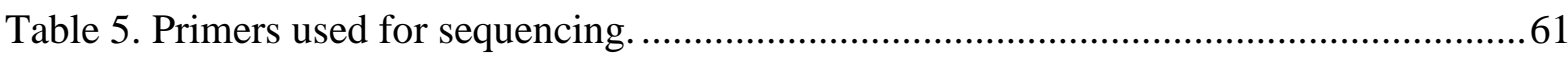

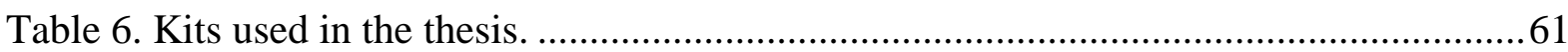

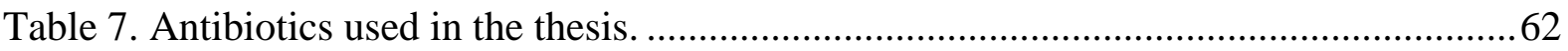

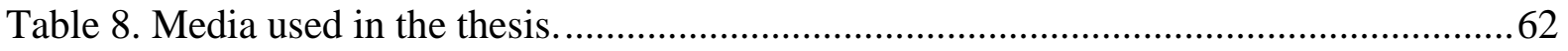

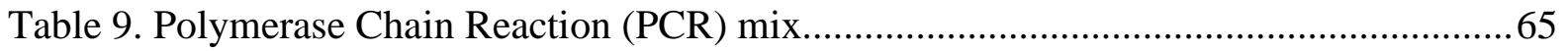

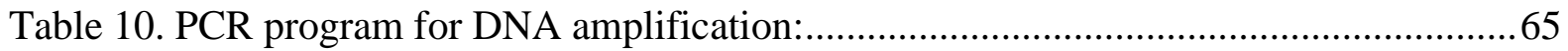

Table 11. PCR program for DNA amplification used for inverse PCR .................................65

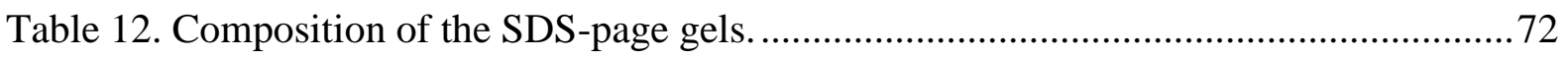

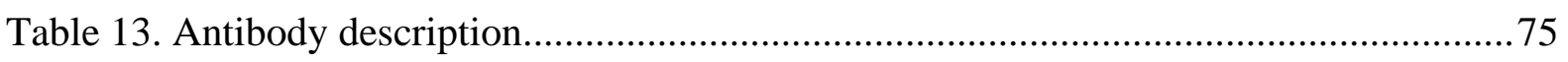

Table 14. Tested conditions for the in vitro assembly of the AcrAB-TolC system............... 108

Table 15. Determination of the efficiency of protein incorporation in the liposome............115

Table 16. Determination of the reconstitution efficiencies and the protein amounts

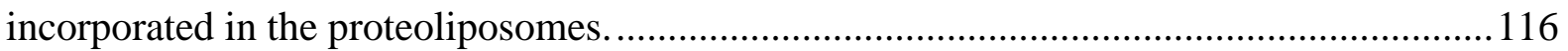

Table 17. Student's t-test analysis on TolC proteoliposomes assay. ..................................... 118

Table 18. The kinetic parameters of the ATPase activity of CopA, gathered during ATP-

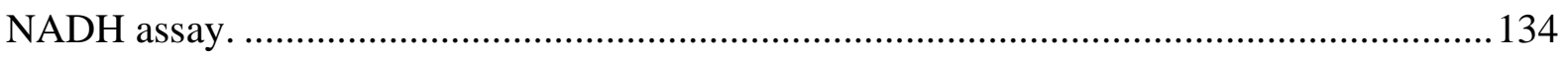

Table 19. Protein amounts in the CopA wild type proteoliposomes. ................................... 138

Table 20. Protein amounts in the CusA wild type proteoliposomes.................................... 149 


\section{$\underline{\text { Declaration }}$}

Except where stated otherwise by reference or acknowledgment, the work presented was generated by myself under the supervision of my advisors during my doctoral studies. All contributions from colleagues are explicitly referenced in the thesis.

The material listed below was obtained in the context of collaborative research:

Figure 71: $\mathrm{Ag}^{+}$-transport into CusA-containing proteoliposomes. (Figure was created by me; This experiment: From the protein production until the activity measurement, was done by Miles Willoughby as a part of his Bachelor thesis (under my supervision)).

Whenever a Figure or Table is identical to a previous publication, it is stated explicitly in the thesis that copyright permission and/or co-author agreement has been obtained.

The following parts of the thesis have been previously published:

The data from Figure 80 were modified and published: Drug susceptibility test of E. coli strains harbouring pRSFDMG_emrA_tolC and p7XC3RH_emrB. (supplementary S3; Published January 2021)

The following figures are based on previous publications:

- $\quad$ Figure 7 was adapted from Figure 4 in (Du \& Luisi, 2015)

- Figure 8 was adapted from Figure 3a in (Ruggerone et al., 2013)

- Figure 9 was adapted from Figure 4a in (Thomas Eicher et al., 2014)

- Figure 11 was adapted from Figure 5 in (Chacon et al., 2014)

- Figure 12 was adapted from Figure 1a and Figure 1b in (Su et al., 2011)

- Figure 18 was adapted from Figure 3 in (Rigaud \& Lévy, 2003)

- Figure 20 was adapted from Figure 1 in (Verchère et al., 2015)

- Figure 21 was adapted from Figure 2a in (Ntsogo Enguéné et al., 2015)

- Figure 58 was adapted from Figure 4 in (Long et al., 2010)

- Figure 72 was adapted from Figure 3 in (Reinke Tobias Müller \& Pos, 2015) 
The following figures and tables were adapted from own publications:

- Figure 74B was taken from Figure 5b in (Yousefian et al., 2021)*

- Figure 80 was adapted from Figure S3 in (Yousefian et al., 2021)

Except those marked (*), all figures were created by myself. 


\section{Acknowledgments}

The past years were a rollercoaster of emotions and events, tests of strength and a war for knowledge. And every person on that pathway had an important role in finding my motivation and achieving my goal. I want to thank everyone in my life for showing me the signs that I had to pass and lessons that I had to learn.

Thank you, Klass Martinus Pos, for being my supervisor and allowing me to finish my $\mathrm{PhD}$ in your laboratory. I am grateful for the experience to be a $\mathrm{PhD}$ student in your group and study interesting topics, as I did. Thank you for the guidance, discussions and help. Your ideas influenced the quality of this work. Thank you for being my mentor.

Thank you, all the members of Pos laboratory, the current and the past ones.

Thank you, Andrea Hermann, for introducing me to the laboratory and helping me practically in my first weeks. I will miss our talks and breaks.

Thank you, my lab mates, Alina Ornik-Cha, Julia Wilhelm, Reinke Müller and Narek Yousefian, for making the $\mathrm{PhD}$ journey a bit easier and a more interesting place to be.

Thank you, Beate Braungart, for the help and assistance during my stay in the laboratory. It was nice to have someone that can always listen and advice you when needed.

Thank you, Eric Geertsma, for the advices and comments during my $\mathrm{PhD}$. Thank you, all Geertsma members, Katharina Holzhüter, Benedikt Kuhn, Nita Chang, Anja Roden and all others, for all the discussions, talks, encouragements and beer hours.

Thank you, Martin Picard, for welcoming me in your laboratory in Paris, for my short but important stay. My thesis was much improved by your comments and advices during these years of knowing you.

Thank you, Igor Štagljar, for your mentoring, listening and supporting for years. It is an honour knowing you.

Thank you, Božidar Đondović. Without your advices, I probably would not be here.

Thank you, my family, for all the energy that you gave me. I inherited a lot from my ancestors, the ones that fought and worked so hard, so that I can be where I am. I am grateful 
to all of them on their momentum and I respect everything that they gave me. Thank you, Tugomir S. Đorđević, tata, for teaching me to be me and to understand the importance of being in love with science and haveing a support in knowledge gathered from books. Thank you, Ivanka V. Đorđević, mama, for giving me the strength when I needed it the most, for showing me how tough I can be and what it means when a parent has faith in a child. Both of you gave me all I need to be who I am. Thank you, Maja T. Đorđević, sestro, for all the talks and planning that we did all these years; you were believing in my vortex before anyone! Thank you for taking care of me and keeping me close to you. We are universal, timeless and limitless!

Thank you, Sascha Marquardt, mužu, mein Ehemann, for all the support, advices, help and encouragement that you gave me. This period would never be the same without you and probably would not end the same if you were not there. Beside you I have been myself and grew stronger to face the challenges. Thank you for letting me do that. Next steps we will write together as well and I am enormously grateful for that, for you, being with me and beside me. You are my rock and my star.

Nothing in life comes easy, but nothing comes without a reason as well. That is why I believe that anyone and everyone that I have ever met in the past years led me to this point, where I can sum up things and close another chapter of my life while starting to write the next one. Sometimes life brings great things, both good and challenging, just to show us that we are strong, capable and more amazing than we could ever imagine. That is my lesson. And I am grateful for that. Life is good, all the time. 


\section{CURRICULUM VITAE}

\section{PERSONAL INFORMATION}

Name:

Date of birth:

Place of birth:

Nationality:

Adress:

Phone number:

E-mail:

Gender:

\section{EDUCATION}

$2017-2021$

$2016-2021$

Jan. 2014
Selena Đorđević-Marquardt

18. September 1986

Pirot, Serbia

Serbian

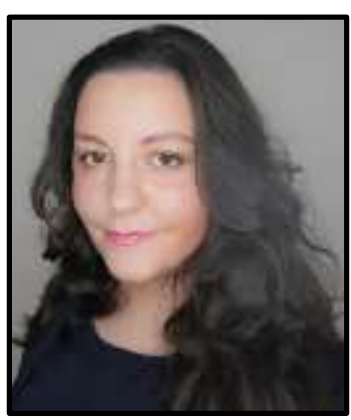

Emil-von-Behring Straße, 81, 60439, Frankfurt am Main

+49015901062693

selenalunanew@gmail.com

Female

\section{Ph.D. in Biochemistry}

Goethe University Frankfurt

Frankfurt am Main, Germany

Thesis title: "Reconstitution and functional characterization of efflux pumps from Escherichia coli"

Supervisor: Prof. Dr. Klaas Martinus Pos

Graduate school - TRAM (TRansport Across Membranes)

Supervisors: Prof. Dr. Klaas Martinus Pos, Prof. Dr. Clemens Glaubitz, Prof. Dr. Robert Tampé

\section{Diploma in Molecular biology and physiology}

University of Belgrade, Serbia

Faculty of Biology

Master/Diploma Thesis: "Metabolic effects of vanadium and cAMP on Phycomyces blakesleeaus"

Supervisors: Prof. Dr. Miroslav Živić 
$2005-2014 \quad$ Studies in Molecular biology and physiology (Diploma)

Specialization: Applied biochemistry

University of Belgrade, Serbia

Faculty of Biology

\section{RESEARCH EXPERIENCE}

$2016-2021$

Ph.D. student at the Membrane Transport Machineries Group

Goethe University Frankfurt

Frankfurt am Main, Germany

Supervisor: Prof. Dr. Klaas Martinus Pos

07.2017 - 07.2017 Laboratoire de biologie physico chimique des protéines membranaires (UMR7099) - Research Internship

Institut de Biologie Physico-Chimique (IBPC)

Paris, France

Supervisor: Dr. Martin Picard

03.2013 - 01.2014 Institute for Biological Research "Siniša Stanković" - Research Internship

Department of Biophysics

Belgrade, Serbia

Supervisor: Prof. Dr. Miroslav Živić

\section{ACADEMIC AWARDS}

May 2005. Best student of generation in high school, Pirot, Serbia; owner of "Tigar AD" company scholarship

\section{MEMBERSHIPS}

$2016-2021$

Integrated Research Training Group - TRAM (TRansport Across Membranes) - Member 


\section{RELEVANT EXPERIMENTAL SKILLS AND HIGHLIGHTS}

Molecular biology methods:

PCR, plasmid purification, cloning, agarose, restriction digests

Biochemical methods:

Protein production, preparation and sterilization of media, reconstitution, enzymatic assays, drug plate dilution assays, polyacrylamide gel electrophoresis, Western blot

Biophysical methods:

Patch clamp, fluorescence measurements

Other:
Manipulation of lipids
Usage of fluorescent probes like a reporter system
Handling complex model systems

\section{GENERAL SKILLS}

Capable working in a team or as individual

Excellent attention to detail

Highly dependable

Excellent communication and written skills

Fast learner

IT Skills: MS Word, MS Excel, MS PowerPoint and general computer skills

Well-developed analytical and numerical ability

Teaching

\section{CERTIFICATIONS}

TRAM graduate school

Workshop on scientific writing - certificate

Workshop - presentation and poster preparation

Workshop - time management 


\section{LANGUAGES}

Serbian (native)

English (fluent)

Spanish

German

French

\section{PUBLICATIONS}

Yousefian, N., Ornik-Cha, A., Poussard, S., Decossas, M., Berbon, M., Daury, L., Taveau, J. C., Dupuy, J. W., Đorđević-Marquardt, S., Lambert, O., \& Pos, K. M. (2021). Structural characterization of the EmrAB-TolC efflux complex from E. coli. Biochimica et biophysica acta. Biomembranes, 1863(1), 183488. https://doi.org/10.1016/j.bbamem.2020.183488 